\title{
Robust Ambient Multisensor Signal Fusion towards Clinical Data Analytics
}

\author{
by \\ Megan Holtzman, M.A.Sc. \\ A Dissertation submitted to \\ the Faculty of Graduate and Postdoctoral Affairs \\ in partial fulfilment of \\ the requirements for the degree of \\ Doctor of Philosophy \\ in Electrical and Computer Engineering
}

Department of Systems and Computer Engineering

Carleton University

Ottawa, Ontario, Canada

April 2014

Copyright (c)

2014 - Megan Holtzman, M.A.Sc. 


\section{Abstract}

As ambient systems proliferate, there is an increased need for data-level fusion methods that reflect the challenges of unknown environments, non-deterministic signal behaviours, and movement artifact. Cushioning between the sensor and bed occupant decreases signal to noise power and signal availability, while non-linear signals can masquerade as delayed and reversed signals. The main contributions of this thesis are to study how these challenges affect extraction of a breathing signal from bed-based sensors and to propose more robust fusion techniques.

New trend analysis methods effectively corrected polarity reversals, increasing the number of good quality signals by $9 \%$ and reducing mean respiratory rate error by 24\%. To fuse these signals, selection combining, weighted summation, and blind source separation methods were innovated and compared. None performed best all of the time; some were generally good with some weaknesses, while others had specialized strengths. Contextual ensemble fusion selected the best fusion method in degraded conditions in $55 \%$ of records, compared to $36 \%$ for the top individual fusion method, providing clinical applications with more reliable data. While sleep medicine is an important application, ambient monitoring is also suited to cognitive medicine and palliative care. Developed methods were applied to monitor patients in palliative care, marking the first long-term, continuous monitoring of this population. Breathing patterns observed in the last weeks of life included Cheyne-Stokes respiration and tachypnea, while breathing variability was associated with survival time. 
To the things that didn't exist before this journey started and now do :

\author{
husband and daddy \\ daughters \\ grandmothers \& grandfathers \\ early mornings \\ iPhones
}

some neural pathways

This is for you. The new and evolving drive this work. 


\section{Acknowledgments}

The work in this thesis could not have been possible without the wisdom and guidance of my supervisors Dr. Rafik Goubran and Dr. Frank Knoefel. I would like to express my great appreciation of their work, support, and time.

I wish to acknowledge the help provided by Jodie Taylor during data collection at Elisabeth Bruyère Hospital, Stéfanie Rocan who ensured the TAFETA project ran smoothly, Cheryl Auclair and Susan Murat in the Dean's Office, Jennifer Poll in the Systems and Computer Engineering Department office, and Daren Russ who fixed my broken computer during the last week of writing this thesis. The support and advice provided by Dr. Ashraf Matrawy and Pat Beirne towards teaching, guidance, and professional endeavors were greatly appreciated.

I thank the Natural Sciences and Engineering Research Council (NSERC), as well Carleton University for their financial assistance, without which this work would not have been possible.

I would like to offer my special thanks to Aaron Holtzman for his support, advice, coaching, and innumerable acts of kindness that kept the home fires burning and kept us from being swept away by the avalanche of debris that accumulated daily. I am grateful to Mary Doody-Jones and Gerry Howell Jones for readily shoveling through the heavy science to proofread my chapters and for giving me my start in the world and appreciation for academics. Thanks to Arabella and Elizabeth Holtzman for reminding me to see the big picture, to best friends Adrienne Boothroyd and Ian 
Dawes for always having our back, lending a hand when we needed one, and helping us through our work/life balance. Thanks to my dragon boat teams for taking me away from my computer to see that hard work, motivation, and learning from mistakes really do pay off.

Finally, I would like to thank my co-researchers in the Digital Processing Laboratory and the wider Department of Systems and Computer Engineering for their assistance, encouragement, and friendship. Some have finished their degrees and moved on, some have been at Carleton almost as long as I, and other are still new to the lab. In particular: Amaya Arcelus, Daphne Townsend, Anita McKee, Vilas Joshi, Jeff Gilchrist, Geoff Green, Idana Veledar, Christophe Herry, Jason Rhinelander, Bruce Wallace, Mike Rockwood, and Stephanie Bennett. 


\section{Statement of Originality}

The work presented in this thesis is the original work of the author, Megan Holtzman (née Megan Howell Jones), developed in the course of fulfilling Ph.D. requirements. My co-supervisors, Rafik Goubran and Frank Knoefel provided guidance and constructive criticism throughout the work. Dr. Goubran is a professor of engineering with the Department of Systems and Computer Engineering at Carleton University, while Dr. Knoefel provided medical input as a physician with Bruyère Continuing Care, in gerontology and cognitive medicine. Part of the work involved collaboration with other students and co-authorship of resulting publications. This collaboration involved working with Amaya Arcelus, Daphne Townsend, and Vilas Joshi.

Collaboration with Amaya Arcelus mainly involved collaborations for monitoring the use of grab bars using pressure sensors. My main contribution to that work was the development of force estimation algorithms, while Dr. Arcelus provided algorithms for location estimation. Given that these algorithms were semi-dependent on each other, we were in frequent consultation. Initial calibration testing and data collection was performed together, as well as setup for testing with participants in studies at the University of Ottawa's School of Rehabilitation Science. I contributed analysis of data for force estimates to the work with older adults in occupational therapy. We were the main author on the papers on which we were the respective first author, but provided critical review for and input into the other papers. The grab bar work comprised these publications: 
- M. Holtzman, A. Arcelus, I. Veledar, R. Goubran, H. Sveistrup, and P. Guitard, "Force Estimation with a Non-Uniform Pressure Sensor Array," in Proc. 2008 IEEE Instrumentation and Measurement Technology Conf. (IMTC 2008), 2008, pp. $1974-1979$.

- A. Arcelus, M. Holtzman, I. Veledar, R. Goubran, H. Sveistrup, and P. Guitard, "Contact Location Estimation from a Nonlinear Array of Pressure Sensors," in Proc. 2008 IEEE Int. Conf. Instrumentation and Measurement Technology (IMTC 2008), 2008, pp. 1969-1973.

- A. Arcelus, M. Holtzman, R. Goubran, H. Sveistrup, P. Guitard, and F. Knoefel, "Analysis of commode grab bar usage for the monitoring of older adults in the smart home environment," in Proc. 2009 Ann. Int. Conf. IEEE Engineering in Medicine and Biology Society (EMBC 2009), 2009, pp. 6155-6158.

Dr. Arcelus helped me at the initial stage of breathing fusion algorithm development by visually analyzing and manually coding different types of breathing segments. This work was of great help towards automated breathing analysis and this next paper on breathing signal fusion. The algorithm in the paper was my sole origination, as well as the code and results analysis. I was the main author, while Dr. Arcelus provided me with constructive editorial input.

- M. Holtzman, A. Arcelus, R. Goubran, and F. Knoefel, "Breathing Signal Fusion in Pressure Sensor Arrays," in Proc. 2008 IEEE Int. Workshop Medical Measurements and Applications (MeMeA 2008), 2008, pp. 71-76.

Dr. Arcelus and I also innovated a system of alarming based on events sensed by ambient sensors in a home environment. We held brainstorming sessions where we both contributed equally. For the paper listed below, Amaya was the main author, and was the sole originator of the parts of that work that did not include this alarming 
system. I also contributed MATLAB code to create pictures of pressure images from sensor data, as well as constructive input on the paper itself.

- A. Arcelus, M. H. Jones, R. Goubran, and F. Knoefel, "Integration of Smart Home Technologies in a Health Monitoring System for the Elderly," in Proc. 21st Int. Conf. Advanced Information Networking and Applications Workshops (AINAW 2007), Niagara Falls, ON, Canada, 2007, vol. 2, pp. 820-825.

Collaborative work with Daphne Townsend focused on breathing analysis for apnea detection. To show that breathing could be validly extracted from unknown mattresses in home environments, we worked together on data collection from many mattress types. I was the main author of the related paper, listed below, to which Dr. Townsend contributed interpretation of step response tests, including writing excerpts and figures. The main analysis and authorship of the work with physiological signals was performed by me.

- M. Holtzman, D. Townsend, R. Goubran, and F. Knoefel, "Validation of Pressure Sensors for Physiological Monitoring in Home Environments," in Proc. 2010 IEEE Int. Workshop Medical Measurements and Applications (MeMeA 2010), 2010, pp. 38-42.

The extraction of breathing from the pressure sensor array was my main focus, and the underlying method of correlation for signal reversal detection presented in the following work had also been part of a previous paper (see "Breathing Signal Fusion in Pressure Sensor Arrays", above). Daphne's contribution was the delay correction that was applied using cross correlation to align signals that were not just reversed, but also delayed with respect to each other. She was the main author of that paper, while the analysis was on the data I had collected from participants through my own research. I provided the signal to noise performance metric, its accompanying 
explanation excerpts in the paper and analysis of the results using the metric. I also contributed help with code development, brainstorming, and paper review.

- D. I. Townsend, M. Holtzman, R. Goubran, M. Frize, and F. Knoefel, "Measurement of Torso Movement With Delay Mapping Using an Unobtrusive PressureSensor Array," IEEE Trans. Instrum. Meas., vol. 60, no. 5, pp. 1751-1760, May 2011.

The two papers on apnea originated from ideas I contributed to Dr. Townsend's work on using the windowed variance of the breathing signals to detect apneas. I had previously written a paper on apnea detection during coursework, where I had used the windowed variance and two other features to detect apneas using fuzzy logic (M. Howell Jones, "Fuzzy Methods for Unobtrusive Apnea Detection," Term Project Report, Intelligent Systems Design, University of Ottawa, Apr. 2007). Dr. Townsend conceived of the apnea model of variance decrease and increase that was used in later work to provide better specificity. I wrote the code that tested the results, based on original code provided by Dr. Townsend. The test data used for the work was from the multi-mattress experiment that we had collected together. The works below were authored by Dr. Townsend with constructive input on results analysis and short excerpts that I authored.

- D. Townsend, M. Holtzman, R. Goubran, M. Frize, and F. Knoefel, "Effect of windowing on central apnea detection," in Proc. 2010 IEEE Int. Workshop Medical Measurements and Applications (MeMeA 2010), Ottawa, ON, 2010, pp. $117-120$.

- D. Townsend, M. Holtzman, R. Goubran, M. Frize, and F. Knoefel, "Relative Thresholding With Under-Mattress Pressure Sensors to Detect Central Apnea," IEEE Trans. Instrum. Meas., vol. 60, no. 10, pp. 3281-3289, Oct. 2011. 
- D. I. Townsend, M. Holtzman, R. Goubran, M. Frize, and F. Knoefel, "Simulated central apnea detection using the pressure variance," in Proc. 2009 Ann. Int. Conf. IEEE Engineering in Medicine and Biology Society (EMBC 2009), 2009, pp. 3917-3920.

Vilas Joshi and I worked together, with Amaya Arcelus, to exemplify a home system that could survive data network delays and outages. The original idea for this work was Dr. Joshi's. Dr. Arcelus and I provided test results, each from our own domains of research, for how the system would affect the analysis of parameters extracted from a pressure sensitive smart home. My work was in the movement detection and breathing rate estimation. Vilas authored the paper, with short excerpts, diagrams, and plots regarding my part of the results contributed by myself. Dr. Arcelus similarly contributed excerpts, diagrams, and plots.

- V. Joshi, M. Holtzman, A. Arcelus, R. Goubran, and F. Knoefel, "Highly survivable bed pressure mat remote patient monitoring system for mHealth," in Proc. 2012 Ann. Int. Conf. IEEE Engineering in Medicine and Biology Society (EMBC 2012), 2012, pp. 268-271. 


\section{Table of Contents}

Abstract $\quad$ ii

Acknowledgments $\quad$ iv

Statement of Originality vi vi

Table of Contents $\quad x i$

List of Tables $\quad$ xvii

List of Figures $\quad$ xviii

List of Acronyms xxiii

List of Symbols $\quad$ xxviii

1 Introduction 1

1.1 Motivation . . . . . . . . . . . . . . . . . 1

1.2 Problem Statement . . . . . . . . . . . . . . . . . . 2

1.3 Objectives and Scope . . . . . . . . . . . . . . . . . 6

1.4 System Overview and Thesis Organization . . . . . . . . . . 8

1.5 Thesis Contributions . . . . . . . . . . . . . . . . 9

1.5.1 Contributions in Ambient System Response Characterization 10

1.5.2 Contributions in Signal Alignment . . . . . . . . . . . . . . 12 
1.5.3 Contributions in Data-Level Physiological Signal Fusion . . . . 13

1.5.4 Contributions in Context-Aware Signal Fusion . . . . . . . . . 15

1.5.5 Contributions to Ambient Sensing in Clinical Care . . . . . . 15

2 Background Review $\quad 18$

2.1 Factors of System Response . . . . . . . . . . . . . . . . . . . . . 18

2.1.1 Sensor Location . . . . . . . . . . . . . . . . . 18

2.1.2 Sensor Modality . . . . . . . . . . . . . . . . . 20

2.2 Ambient Sensor Signal Processing . . . . . . . . . . . . . . . . 21

2.2.1 Breathing Effort Signals . . . . . . . . . . . . . . 22

2.2.2 Signal Conditioning . . . . . . . . . . . . . . . 22

2.2.3 Reversal Detection and Correction . . . . . . . . . . . . 23

2.2.4 Breathing Signal Fusion . . . . . . . . . . . . 25

2.2.5 Coping with Movement . . . . . . . . . . . . . 32

2.3 Clinical Applications of Bed-Based Sensing . . . . . . . . . . . . . . 35

2.4 Summary . . . . . . . . . . . . . . . . . 36

3 Experimental Setup and Data Collection 38

3.1 Sensor Technology . . . . . . . . . . . . . . . . . . . . . . . . . 38

3.1.1 Pressure Sensor Mats . . . . . . . . . . . . . . . . . . . 38

3.1.2 Braebon Respibands . . . . . . . . . . . . . . . 39

3.2 Data Acquisition and Analysis Software . . . . . . . . . . . . 40

3.2.1 Acquisition and Data Conversion . . . . . . . . . . . . . . 40

3.3 Experimental Data . . . . . . . . . . . . . . 41

3.3.1 Ethics Approval . . . . . . . . . . . . . . . 41

3.3.2 Multi-mattress Experiment . . . . . . . . . . . . . . 42

3.3.3 Multi-Participant Experiment . . . . . . . . . . . 43

3.3.4 Experimental Data Sets . . . . . . . . . . . 45 
3.4 Evaluation of Fused Respiratory Signals . . . . . . . . . . . . . . . . 48

3.4.1 Comparison to Respiband Signals . . . . . . . . . . . . . . . . 49

3.4.2 Comparison to Respiband Respiratory Rates . . . . . . . . . . 51

4 Characterization of System Response 53

4.1 Introduction . . . . . . . . . . . . . . . . . 53

4.2 Experiment \#1: Environmental Response of Unknown Signal Dampening 54 4.2 .1 Results ..................... 55

4.2 .2 Discussion . . . . . . . . . . . . . . . 57

4.3 Characterization of Non-Uniform Sensor Array Response . . . . . . . 58

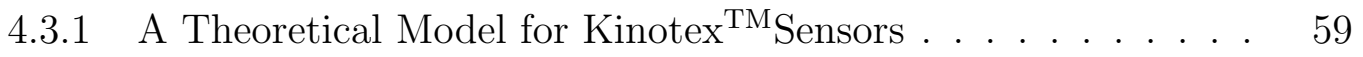

4.3.2 Method: Calibrating with Non-Uniform Spatial Response . . . 61

4.4 Experiment \#2: Linearization of Non-Uniform Sensor Arrays . . . . . 63

4.4.1 Results: Calibration ................. 65

4.4.2 Results: Consequences for Breathing Fusion . . . . . . . . . . 66

4.4.3 Discussion . . . . . . . . . . . . . . . . . . . . . 68

4.5 Summary . . . . . . . . . . . . . . . . . 69

$\begin{array}{lll}5 & \text { Reversal Detection } & 71\end{array}$

5.1 Introduction . . . . . . . . . . . . . . . . . 72

5.2 Multisensor Reversal Detection Signal Model . . . . . . . . . . . . 73

5.3 Methods of Reversal Detection . . . . . . . . . . . . . . . . . . 74

5.3.1 Correlation Analysis for Reversal Detection . . . . . . . . . . 74

5.3.2 Decision Directed Slope Reversal Detection . . . . . . . . . . . 77

5.3.3 Selected Reference Slope Reversal Detection . . . . . . . . . . 81

5.3.4 Adaptive Trend Analysis for Reversal Detection . . . . . . . . 82

5.4 Experiment \#1: Simulation . . . . . . . . . . . . 84

5.4.1 Testing Protocol .................. 85 
5.4 Parameter Selection . . . . . . . . . . . . . . . 86

5.4.3 Simulation Analysis . . . . . . . . . . . . . . . . . . . 90

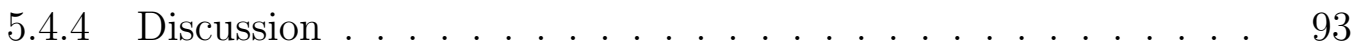

5.5 Experiment \#2: Breathing Signal Reversal Detection . . . . . . . . . 95

5.5.1 Testing Protocol . . . . . . . . . . . . . . . 95

5.5.2 Results: Parameter Selection . . . . . . . . . . . . . 96

5.5.3 Results: Movement Corruption . . . . . . . . . . . . . 97

5.5.4 Results: Validation . . . . . . . . . . . . . . . . 98

5.5.5 Discussion ..................... 102

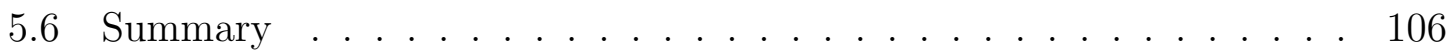

6 Respiratory Signal Fusion by Linear Combining 107

6.1 Introduction . . . . . . . . . . . . . . . . . 108

6.2 Methods . . . . . . . . . . . . . . . . . . 108

6.2.1 Spectral Parameter Estimation . . . . . . . . . . . . . 109

6.2.2 Methods of Selection Combining . . . . . . . . . . . . . . 111

6.2.3 Methods of Weighted Summation . . . . . . . . . . . . . . . . 112

6.2.4 Methods of Blind Source Separation . . . . . . . . . . . . . . 116

6.3 Experiment \#1: Signal-Level Fusion in Adverse Conditions . . . . . . 118

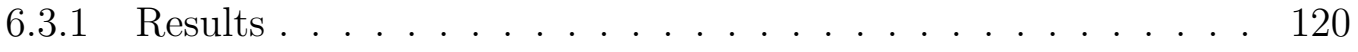

6.3 .2 Discussion ..................... 127

6.4 Experiment \#2: Validation of Experimental Results . . . . . . . . . . 130

6.4.1 Results...................... 130

6.4 Discussion ..................... 134

6.5 Summary . . . . . . . . . . . . . . . . . . 135

7 Ensemble Fusion by Environmental Context Awareness 137

7.1 Introduction . . . . . . . . . . . . . . . . . . . . 138 
7.2 Method: Ensemble Signal Fusion using Environmental and Signal Con-

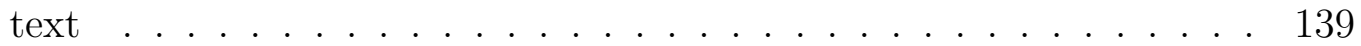

7.2 .1 Contextual Features . . . . . . . . . . . . . . . . 140

7.2 .2 Classification . . . . . . . . . . . . . . . . . . . . 141

7.3 Optimization . . . . . . . . . . . . . . . . . . . 142

7.3.1 Fusion Agent Optimization . . . . . . . . . . . . . . . . . 142

7.3.2 Classifier Optimization . . . . . . . . . . . . . . . . . . 143

7.4 Experiment . . . . . . . . . . . . . . . . . . . . . . . 144

7.4 .1 Results . . . . . . . . . . . . . . . . . . . 145

7.4 .2 Discussion . . . . . . . . . . . . . . . . . . 152

7.5 Summary . . . . . . . . . . . . . . . . . . . . 155

8 Applications in Clinical Care 156

8.1 Incorporating Ambient Technology into Clinical Care . . . . . . . 157

8.1.1 Applications in Cognitive Medicine . . . . . . . . . . . . . . 158

8.1.2 Monitoring in Palliative Care . . . . . . . . . . . . . 159

8.2 Experiment \#1: Motion Monitoring in Palliative Care . . . . . . . 161

8.2 .1 Methods . . . . . . . . . . . . . . . . . . . . . . 161

8.2 .2 Results. . . . . . . . . . . . . . . . . 165

8.2 .3 Discussion . . . . . . . . . . . . . . . . . . . 170

8.3 Experiment \#2: Long-term Monitoring in Palliative Care: A Pilot Study171

8.3 .1 Methods . . . . . . . . . . . . . . . . . . . . . . . . 172

8.3 .2 Results . . . . . . . . . . . . . . . . . . . . 181

8.3 .3 Discussion . . . . . . . . . . . . . . . . . . . 186

8.4 Summary . . . . . . . . . . . . . . . . . . . . . . . . 188

9 Conclusions 190

9.1 Summary of Research . . . . . . . . . . . . . . . . . . . . 190 
9.1.1 Summary of Research Results: Chapter 4 . . . . . . . . . . . . 191

9.1.2 Summary of Research Results: Chapter 5 . . . . . . . . . . . . 192

9.1.3 Summary of Research Results: Chapter 6 . . . . . . . . . . . . 192

9.1.4 Summary of Research Results: Chapter 7 . . . . . . . . . . 193

9.1.5 Summary of Research Results: Chapter 8. . . . . . . . . . . . 194

9.2 Conclusions . . . . . . . . . . . . . . . . . . . . . . . . . 194

9.3 Suggestions for Future Work . . . . . . . . . . . . . . . 196

$\begin{array}{ll}\text { List of References } & 200\end{array}$

Appendix A Background Information on Palliative Care 225

A.1 Overview of Palliative Care . . . . . . . . . . . . . . 225

A.2 Palliative Care Clients . . . . . . . . . . . . . . . . . . . . 228

A.3 End of Life Symptoms . . . . . . . . . . . . . . . . . . . . . . . 229

A.4 Palliative Home Care . . . . . . . . . . . . . . . . . . . 230 


\section{List of Tables}

3.1 Multi-mattress experiment: Mattress types and thicknesses . . . . . 43

3.2 Multi-participant experiment: Participant information . . . . . . . . . 44

4.1 Error statistics for given methods . . . . . . . . . . . . 66

5.1 Performance of reversal correction applied to validation records . . . 99

5.2 Statistics of respiratory rate estimation after reversal corrections . . . 102

6.1 Performance of breathing fusion methods applied to validation records 131

6.2 Statistics of respiratory rate estimation from signals fused using Adapt

RD, listed by participant . . . . . . . . . . . . . . . 134

7.1 Evaluation of fusion agents from training data . . . . . . . . . . 147

7.2 Performance of fusion methods applied to the test sets . . . . . . . 149

7.3 Statistics of respiratory rate estimation from signals fused using ensemble fusion with contextual awareness for the multi-participant experiment151

8.1 Threshold parameter settings . . . . . . . . . . . . . 163

8.2 Detection statistics for position shifts $(\%) \ldots \ldots \ldots 7$

8.3 Detection statistics for movement . . . . . . . . . . . . . 168

8.4 Summary of participants for long-term monitoring in palliative care . 174 


\section{List of Figures}

1.1 Block diagram of ambient sensor array monitoring to extract a source signal from an array of sensors . . . . . . . . . . . . . 4

1.2 The sensorized bed can be used for prevention, screening, and monitoring 7

1.3 The system architecture for source signal extraction . . . . . . . 9

2.1 Possible locations of bed-based sensors . . . . . . . . . . . . . . 19

2.2 Block diagram of respiratory fusion as linear diversity combining . . . 27

2.3 Block diagram of sensor selection as selection combining . . . . . 27

2.4 Block diagram of sensor summation as equal gain combining . . . . 29

2.5 Block diagram of blind source separation as linear combining . . . . 30

2.6 Conventional breathing analysis architecture . . . . . . . . . . 33

3.1 S4 Sensors BOS mat . . . . . . . . . . . . . . . . . . . 39

3.2 Respiband setup: band, interface box, and data acquisition board . . 40

3.3 Components in the multi-mattress experiment . . . . . . . . 42

3.4 Components of the multi-participant experiment $\ldots \ldots \ldots \ldots 44$

3.5 Data sets extracted from participant data $\ldots \ldots \ldots \ldots$

3.6 Data sets created by artificial corruption of clean respiratory epochs . 47

4.1 Ten seconds of typical respiratory and cardiac signals recorded from above and below the deluxe mattress $\ldots \ldots \ldots \ldots \ldots$

4.2 Signal power loss with respect to thickness and mattress material . . 56 
4.3 Boxplot representing the percentage of sensors with physiological signals above and below the mattress . . . . . . . . . . . 56

4.4 Ideal response of sensors in an array (top) and response of actual sensors in non-uniform pressure sensor array (bottom) . . . . . . . . . 58

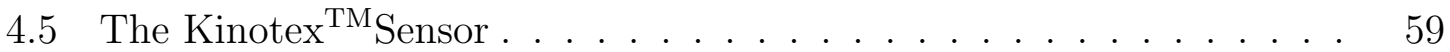

4.6 Block diagrams of linearization through modeled calibration data . . 61

4.7 Top and cutaway views of the array . . . . . . . . . . . 63

4.8 Forward and inverse models of sensor response. Left: theoretical responses along the bar. Right:Examples of inverse modeling . . . . . 65

4.9 Effect of sensor response on the shape of a simulated breath signal . . 67

5.1 Two detrended respiratory signals from a pressure sensor array with similar amplitude but reversed polarity . . . . . . . . . . . . . . . . 72

5.2 Pearson's correlation coefficient as a standardized linear regression . . 75

5.3 Sensor signals affected by movement . . . . . . . . . . . . . 76

5.4 Pearson's correlation coefficient as a standardized linear regression during movement . . . . . . . . . . . . . . . 76

5.5 The DDSRD method uses the linear trends $\tau_{k}\left(t_{n}\right)$ to iteratively refine $p_{k} 78$

5.6 Normalized slopes and voted direction during movement . . . . . . 80

5.7 Reversals corrected by the short-term DDSRD . . . . . . . . . . . 81

5.8 Block diagram of online adaptation for polarity weight $w_{1}\left(t_{n}\right) \quad \ldots$. . 82

5.9 Examples of weight and polarity sign adaptation for the Adapt RD

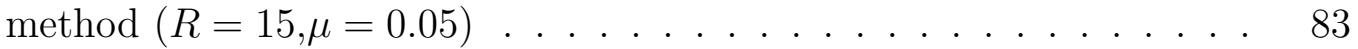

5.10 Large array example adaptation, including noisy signals and the $p_{k}=0$ case on some sensors . . . . . . . . . . . . . . . . . 84

5.11 Simulated signals with varying parameters . . . . . . . . . 87

5.12 Accuracy as a function of window length parameter $\mathrm{R}$ in the presence of noise and/or drift . . . . . . . . . . . . . . . . . . . 88 
5.13 Accuracy of polarity reversal detection as a function of window length parameter $R$, varying the period of the underlying signal in strong noise and drift . . . . . . . . . . . . . . . . . . .

5.14 Reversal detection accuracy respibands as a function of learning rate $\mu$, varying window length $R \ldots \ldots \ldots$

5.15 Effects of SNR on mean percentage of correct polarities: (a) Low Drift (b) High Drift . . . . . . . . . . . . . . . . . . . . . . 90

5.16 Effects of interference on mean percentage of correct polarities: (a) Drift (b) Burst Interference . . . . . . . . . . . . . . . . . 91

5.17 Effect of reference fusion on polarity accuracy . . . . . . . . . . . . . 92

5.18 Effect of non-stationary periods on accuracy of correct polarities (low

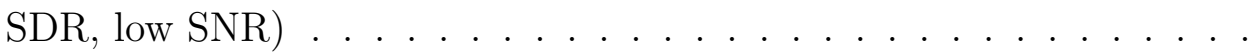

5.19 All experimental training data: accuracy according to window length parameter $R$. Basic and filtered pre-conditioning schemes shown for non-adaptive methods, $\mu$ for the adaptive method. . . . . . . . . . . . 97

5.20 Effect of movement corruption on signal quality . . . . . . . . . . . . 98

5.21 Boxplots of output signal quality by method . . . . . . . . . . . . . . 99

5.22 Bland-Altman plots of extracted respiration rates comparing respiband and ambient sensors for Spec. Phase and Adapt RD methods . . . . . 100

5.23 Respiratory rate agreement with respibands by reversal detection

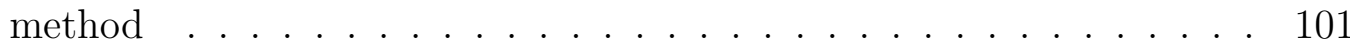

6.1 Locating the signal and the noise components in the PSD . . . . . . . 110

6.2 Block diagram of spectral ratio fitness calculator for selection combining111

6.3 Block diagram of method to calculate MRC gain from variance . . . . 113

6.4 Block diagram of method to calculate MRC gain from power spectral density . . . . . . . . . . . . . . . . . 115

6.5 Block diagram of MRC (PSD+EGC) method . . . . . . . . . . 115 
6.6 Block diagram of PCA with component selection . . . . . . . . . . . . 117

6.7 Block diagram of ICA using PCA and selection combining . . . . . 118

6.8 Mean correlation coefficient of method outputs compared to respibands by percentage of added noise corruption . . . . . . . . . . . . . . 121

6.9 Mean correlation coefficient of method outputs compared to respibands by percentage of added drift corruption . . . . . . . . . . . . . . . . 122

6.10 Mean correlation coefficient of method outputs compared to respibands by duration of movement . . . . . . . . . . . . . . . . . . . . 123

6.11 Mean correlation coefficient of method outputs compared to respibands by number of sensors with valid breathing signals . . . . . . . . . . . 125

6.12 Mean correlation coefficient of method outputs compared to respibands by amount of period variability . . . . . . . . . . . . . 126

6.13 Barcharts of respiratory rate agreement with respibands by fusion

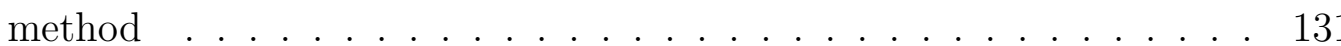

6.14 Boxplots of output signal quality by fusion method . . . . . . . . . . 132

6.15 Bland-Altman plots of extracted respiration rates comparing respiband and ambient sensors for Adapt RD and MRC (PSD+EGC) methods .

7.1 Environmental conditions affecting fusion methods and its output signal quality . . . . . . . . . . . . . . . . . . 138

7.2 Block diagram of ensemble fusion using environmental and signal context139

7.3 Classification accuracy drops as the number of fusion agents increases, but output signal quality improves - up to a point. . . . . . . . . . . 145

7.4 SVM parameter selection: Classification accuracy and signal quality according to cost and $\gamma \ldots \ldots \ldots \ldots$. . . . . . . . . . . . . 148

7.5 Output signal quality with respect to classification accuracy . . . . . 148

7.6 Confusion matrices for selected agents performance over the test sets 150

8.1 A sensorized bed for ambient care . . . . . . . . . . . . . . 157 
8.2 A finite state machine for segmenting data by bed occupant status . . 162

8.3 Pie chart of percentage of time spent performing activities according to annotations . . . . . . . . . . . . . . . . 166

8.4 Pie chart of the percentage of activities independently performed . . . 166

8.5 Detected and annotated limb movements . . . . . . . . . . . . . . 169

8.6 Two interesting pressure sensor patterns of movement discovered in the data . . . . . . . . . . . . . . . . . 170

8.7 Diagram of the installation of sensor mats in the palliative care ward 172

8.8 Photograph of actuated bed frame with contoured panels . . . . . . . 173

8.9 Collected Data from participants stay in palliative care . . . . . . . 175

8.10 An example of Cheyne-Stokes respiration from a participant with brain cancer . . . . . . . . . . . . . . . . 177

8.11 Proposed signal processing architecture . . . . . . . . . . . . . . . . 178

8.12 Percentage of valid epochs using a given quality threshold . . . . . . . 182

8.13 Examples of breathing patterns observed in the extracted respiratory signal of palliative care participant data . . . . . . . . . . . . . . . 183

8.14 Scatter plot of hourly $\sigma_{\mathrm{RI}}$ and $\sigma_{\mathrm{RA}} \ldots \ldots \ldots$. . . . . . . . . . . . 183

8.15 Boxplots of hourly respiratory parameters according to lung involvement 184

8.16 Mean respiratory parameters according to number of days from mortality 185 


\section{List of Acronyms}

\begin{tabular}{ll}
\hline Acronyms & Definition \\
\hline \hline Adapt RD & Adaptive Reversal Detection \\
ADC & Analog to Digital Converter \\
AHI & Apnea Hypopnea Index \\
AIDS & Acquired Immune Deficiency Syndrome \\
ANN & Artificial Neural Network \\
AWGN & Additive White Gaussian Noise \\
BCG & Ballistocardiogram \\
BOS & Bed Occupancy Sensor \\
BPM & Breaths Per Minute \\
BSS & Blind Source Separation \\
CHF & Congestive Heart Failure \\
COPD & Confidence Interval for a X\% Confidence Level \\
\end{tabular}




\begin{tabular}{|c|c|}
\hline CSR & Cheyne-Stokes respiration \\
\hline CSV & Comma Separated Values \\
\hline DDSRD & Decision Directed Slope Reversal Detection \\
\hline SRSRD & Selected Reference Slope Reversal Detection \\
\hline EER & Equal Error Rate \\
\hline EGC & Equal Gain Combining/Combiner \\
\hline EMD & Empirical Mode Decomposition \\
\hline ICA & Independent Component Analysis \\
\hline IP & Inductance Plethysmography \\
\hline FDA & (US) Food and Drug Administration \\
\hline FIR & Finite Impulse Response \\
\hline $\mathrm{FP}$ & False Positive \\
\hline FN & False Negative \\
\hline FSR & Force Sensitive Resistor \\
\hline GI & Gastro-Intestinal \\
\hline GLR & Generalized Likelihood Ratio \\
\hline GU & Geneto-Urinary \\
\hline GUI & Graphical User Interface \\
\hline HRV & Heart Rate Variability \\
\hline
\end{tabular}




\begin{tabular}{|c|c|}
\hline LMS & Least Mean Square \\
\hline LDC & Linear Diversity Combining \\
\hline LUT & Look Up Table \\
\hline $\mathrm{MCC}$ & Matthews Correlation Coefficient \\
\hline MCI & Mild Cognitive Impairment \\
\hline MEF & Movement Energy Fraction \\
\hline MRC & Maximal Ratio Combining/Combiner \\
\hline $\mathrm{MRC}(\mathrm{PSD})$ & Maximal Ratio Combining/Combiner using Power Spectral Density \\
\hline $\mathrm{MRC}(\mathrm{PSD}+\mathrm{EGC})$ & Maximal Ratio Combining/Combiner using Power Spectral Density and Eq \\
\hline MRC (Var) & Maximal Ratio Combining/Combiner using Variance \\
\hline MSE & Mean Square Error \\
\hline NPV & Negative Predictive Value \\
\hline NREM & Non Random Eye Movement (sleep stage) \\
\hline $\mathrm{PB}$ & Periodic Breathing \\
\hline PCA & Principle Component Analysis \\
\hline PPM & Parts Per Million \\
\hline PPV & Positive Predictive Value \\
\hline PSD & Power Spectral Density \\
\hline PVDF & Polyvinylidene Fluoride \\
\hline
\end{tabular}




$\begin{array}{ll}\text { RA } & \text { Respiratory Amplitude } \\ \text { RAID } & \text { Respiratory Amplitude and Interval Disturbance } \\ \text { RBF } & \text { Radial Basis Function } \\ \text { RDI } & \text { Respiratory Disorder Index } \\ \text { REB } & \text { Research Ethics Board } \\ \text { REM } & \text { Rapid Eye Movement (sleep stage) } \\ \text { RIP } & \text { Respiratory Inductance Plethysmography } \\ \text { RMS } & \text { Root Mean Square } \\ \text { RMSE } & \text { Root Mean Square Error } \\ \text { ROC } & \text { Receiver Operating Characteristic } \\ \text { RRI } & \text { Singular Value Decomposition } \\ \text { SVI. (SNR-PSD) } & \text { Selection combining using Power Spectral Density and Signal to Noise Rati } \\ \text { SCSB } & \text { Signal to Noise Ratio } \\ \text { SDR } & \text { Static Charge Sensitive Bed } \\ \text { Sel. (PSD) } & \text { Signal to Drift Ratio } \\ & \text { Selection combining using Power Spectral Density } \\ \text { Selion combining using Signal to Noise Ratio }\end{array}$


SVM

TDE

$\mathrm{TN}$

TP

USB

WHO
Support Vector Machine

Time Delay Estimation

True Negative

True Positive

Universal Serial Bus

World Health Organization 


\section{List of Symbols}

\begin{tabular}{|c|c|}
\hline Symbols & Definition \\
\hline$a$ & normalized sample numbers \\
\hline$A$ & area \\
\hline$a_{c}$ & foam compliance \\
\hline$\alpha$ & absorption coefficient \\
\hline$b(j, n)$ & $\begin{array}{l}\text { data point } j \text { in a window of data centered around sample } \\
n\end{array}$ \\
\hline $\mathrm{CI}(\bar{r})_{X}$ & $\begin{array}{l}\text { Confidence interval of the mean of } r \text { for a } \mathrm{x} \% \text { confidence } \\
\text { Level }\end{array}$ \\
\hline$c$ & contextual agent number \\
\hline C & total number of contextual agents used \\
\hline$\left(c_{\text {low }}, c_{\text {high }}\right)$ & Confidence interval range \\
\hline$d(t)$ & drift signal \\
\hline$e_{k}\left(t_{n}\right)$ & error signal for sensor $k$ at sample time $t_{n}$ \\
\hline E & length of an epoch or segment \\
\hline
\end{tabular}




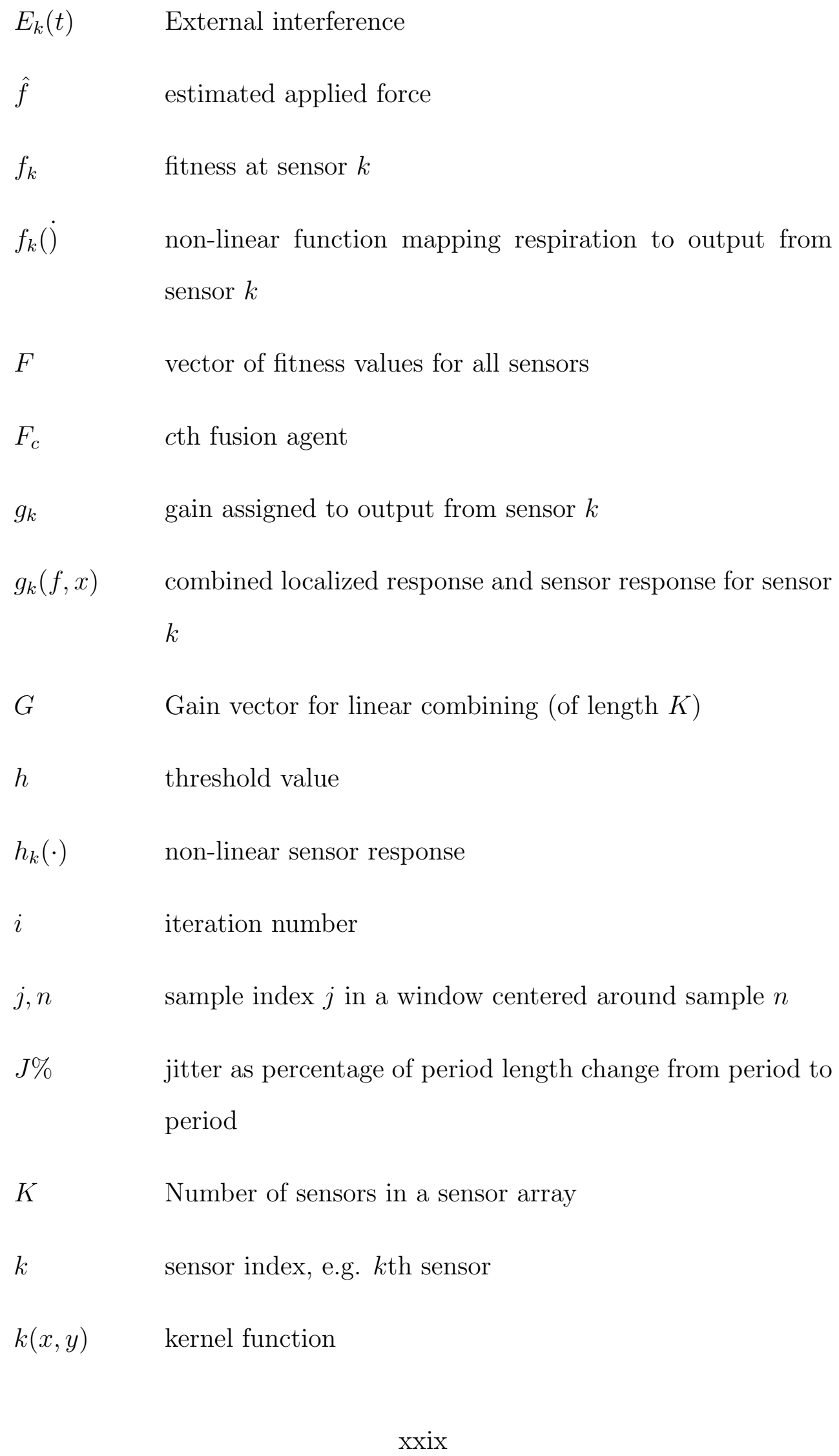




\begin{tabular}{|c|c|}
\hline$l$ & location of applied force \\
\hline$l_{s}(\cdot)$ & localized response \\
\hline$L$ & window size for movement detector \\
\hline $\mathrm{m}_{k}^{\prime}\left(t_{n}\right)$ & corrected slope of sensor $k$ at sample time $t_{n}$ \\
\hline$\mu$ & learning rate of LMS algorithm \\
\hline$n$ & sample index \\
\hline$n_{k}(t)$ & noise signal \\
\hline$n_{k, \mathrm{ADC}}(t)$ & analog to digital conversion noise signal \\
\hline$N$ & Noise power \\
\hline$N_{s}$ & Number of samples in a segment or file \\
\hline$N_{e}$ & Number of epochs or data segments \\
\hline$N_{0}$ & Noise spectral density \\
\hline$N_{\text {equal }}$ & Number of samples that are equal in testing for a trend \\
\hline$N_{\text {higher }}$ & Number of samples that are higher in testing for a trend \\
\hline$N_{\text {lower }}$ & Number of samples that are lower in testing for a trend \\
\hline$N_{\mathrm{SRC}}$ & Number of signal sources (blind source separation) \\
\hline$\phi$ & Phase angle \\
\hline$p$ & applied pressure \\
\hline$p_{k}$ & polarity reversal $\in-1,0,1$ of sensor $k$ \\
\hline
\end{tabular}




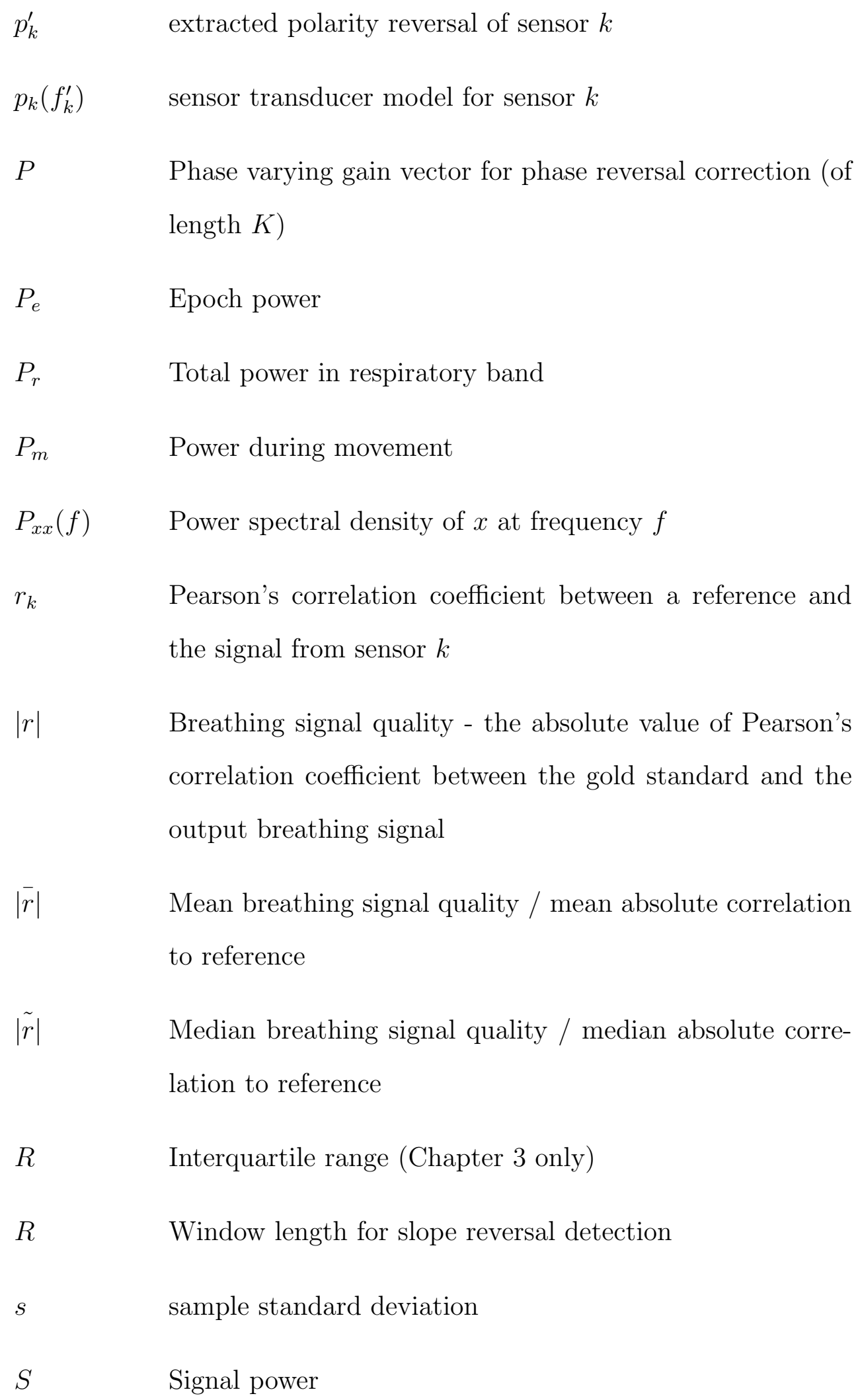


$S_{\text {shift }} \quad$ Minimum number of sensors changing required to declare a position shift

$S_{\text {occup }} \quad$ Minimum number of loaded or active sensors required to declare occupancy

$\sigma_{N L} \quad$ non-linearity factor

$\tau_{k}\left(t_{n}\right) \quad$ trend of sensor $k$ at sample $t_{n}$

$\tau_{\mathbf{k}} \quad$ trend of sensor $k$ over a segment

$\overline{\tau_{k}\left(t_{n}\right)} \quad$ normalized trend of sensor $k$ at sample $t_{n}$

$\overline{\tau_{\mathbf{k}}} \quad$ normalized trend of sensor $k$ over a segment

$\tau_{\mathbf{k}}^{\prime} \quad$ reversal corrected normalized trend of sensor $k$ over a segment

$t_{0} \quad$ initial thickness

$t_{f} \quad$ thickness

$\overline{t_{f}} \quad$ normalized thickness

$t_{\text {act }} \quad$ Large threshold

$t_{\text {large } \quad \text { Large threshold }}$

$t_{\text {med }} \quad$ Medium threshold

$t_{\text {sum }} \quad$ Sum threshold

$t_{\text {zero }} \quad$ Zero threshold for occupancy loading

$t_{m} \quad$ thickness of mattress 


$\begin{array}{ll}t_{0} & \text { Uncompressed (initial) thickness } \\ t_{s}(x, f) & \text { transfer of force to location } x \text { of sensor } \\ t & \text { time } \\ t_{n} & \text { time at sample } n \\ t_{0} & \text { Start time: time of the first sample } \\ t_{l} & \text { End time: time of the last sample } \\ x_{k}(t) & \text { sensor input at sample time } t, \text { sensor } k \\ \mathbf{x}_{\mathbf{r}} & \text { reference signal } \\ x_{r}\left(t_{n}\right) & \text { reference signal at sample time } t_{n} \\ v_{k} & \text { baseline DC } \\ z_{L}^{\prime}(t) & \text { Window length } \\ w_{m} & \text { Window length } \\ & \end{array}$




\section{Chapter 1}

\section{Introduction}

\subsection{Motivation}

Ambient sensors pervade our lives. These sensors, embedded into our everyday environments, monitor and respond to our needs without interaction on our part [1], [2]. An ambient intelligence system interprets events occurring in the environment, represents this knowledge, plans actions around events, interacts with humans, and takes action based on the events [3]. Ambient systems can be very unobtrusive since the sensors can be inconspicuous and the system designed for only limited intervention from users. Indeed, they can be forgotten until they are needed.

Ambient intelligence is so commonplace that we hardly marvel at a traffic signal that automatically changes to green when cars are waiting, a counter installed in a bike lane to record daily usage, or a toilet that flushes itself. Perhaps we do not marvel at these since all of these technologies produce signals that are generally simple to interpret. Binary presence is inferred from a high or low signal and an action proceeds once the signal passes a certain threshold. We have yet to harness the full potential of ambient sensor monitoring, when sensors will detect and respond to much more complicated signals.

For example, ambient systems can be used to identify people according to their 
gait [4] or summon emergency vehicles and contact friends and family when they detect medical events like heart attacks or strokes [5]. Moreover, intelligent sensors around us could learn our personal patterns and warn us when they sense a change, before we are even aware of danger.

By employing not just one sensor but many sensors in a multisensor array configuration, the quality of the extracted signals can be enhanced by making use of redundant information [6]. An array of sensors also provides localization capability and thus more monitoring opportunities.

Opportunities for ambient sensing abound. Even the single sensing modality of pressure sensing provides many possibilities. Most surfaces, including furnishings and floors, can be covered by pressure sensors [7]. Once embedded in the environment, pressure sensors can be used to detect presence, monitor weight, quantify movement strength and duration, and extract cardiorespiratory activity. By using a multisensor array of pressure sensors instead of a single sensor, potential exists to improve weak cardiorespiratory signals, localize motion, and infer posture.

\section{$1.2 \quad$ Problem Statement}

Reliability and robustness has been identified as a challenge for sensors operated for medical monitoring in home environments [8], [9]. To be "robust" is to be "able to withstand or overcome adverse conditions" [10]. Intelligent and robust processing of sensor data is critical to the adoption of ambient sensorized systems. Inadequate processing leads to poor signal quality, inaccurate information gleaned from poor

quality signals, faulty decisions made from inaccurate information, and ultimately abandonment of the unreliable monitoring system. Current analysis techniques are often inadequate to deal with naturally occurring signals in their uncontrolled and 
chaotic environments. Even in more regimented laboratory environments, computerization has yet to replace the drudgery of manual scoring. For instance, best practices for the evaluation of sleep apnea is laboratory polysomnography [11], which calls for visual scoring by a trained expert [12]. Yet, we should expect not just to replace visual scoring, but to improve it by taking advantage of features that are not detectable to the human eye [13].

The barriers to automated medical monitoring stem partly from conditions of interference in the sensor system itself and partly from the non-deterministic nature of the physiological signals. Although multisensor systems provide signal redundancy, they also increase system complexity and can obscure signal analysis by introducing new interference and differing localized and sensor responses. If signal fusion is poorly handled, the resulting output could less accurately reflect the source signal than a system based on just a single sensor.

Fig. 1.1 depicts the ambient multisensor fusion problem for a system with $K$ sensors. Monitoring of a non-stationary, quasiperiodic source signal with unknown and variable amplitude is desired. This signal is modeled as a periodic signal with temporal jitter, shifting the period by up to $\pm T_{j}$ seconds from one cycle to the next. The quasiperiodic signal is fed to each sensor in the array, where its localized behaviour shapes the signal response $l_{k}(\cdot)$ of the $k$ th sensor. Interference $E(t)$ from other sources in the environment is added to the signal, with each sensor experiencing this interference as a unique $E_{k}(t)$. Baseline levels $v_{k}$ vary from sensor to sensor, since each sensor's baseline value may differ according to the location of the sensor in the environment, its base load, and natural variations in the sensor's characteristics. The sensor itself contributes unique combinations of noise and drift signals, $n_{k}(t)$ and $d_{k}(t)$ respectively. Sensor responses $h_{k}(\cdot)$ may be non-linear and variable between sensors. Quantization noise $n_{k, \mathrm{ADC}}(t)$ is added by the analog to digital converter (ADC). The final input signal $x_{k}\left(t_{n}\right)$ is a sampled version of the original source signal $z(t)$ with 


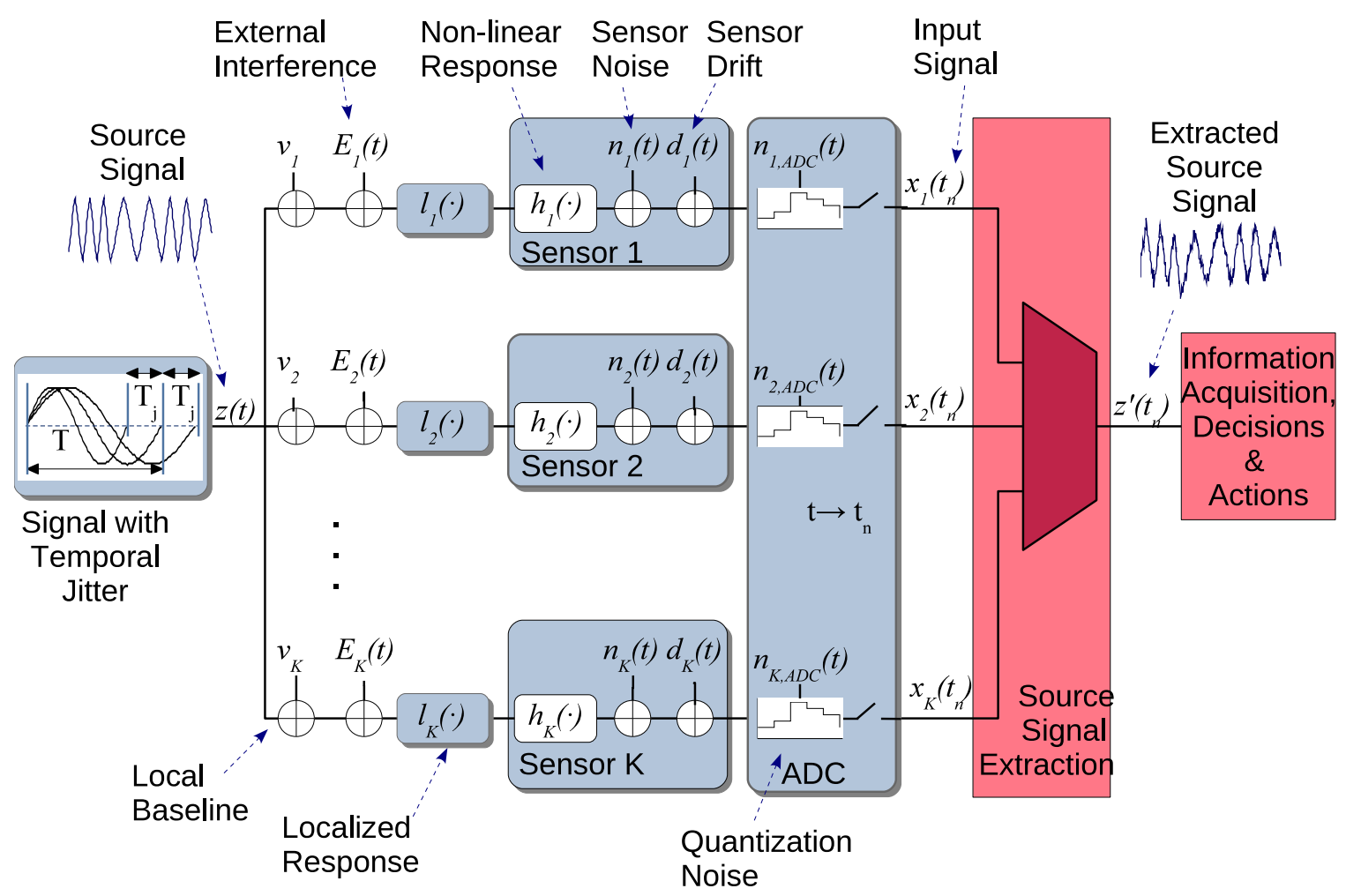

Figure 1.1: Block diagram of ambient sensor array monitoring to extract a source signal from an array of sensors. Complications arise from non-stationary signal behaviour (temporal jitter), system response (non-linear sensor response and localized environmental response), and interference (external interference from other sources, noise from the sensor, quantization noise, and sensor drift).

added interference, drift, and noise.

The effect of the complexities arising by using multiple sensors is exemplified in the evolving use of bed-based sensor arrays. Early bed-based sensing in the 1980's by Alihanka et al. used a specialized mattress providing a single sensor signal for movement and cardiopulmonary monitoring [14]. As the benefit of such ambient monitoring was recognized, multisensor systems emerged in the late 1990's and early 2000's that used 30 to 220 sensors embedded into mattresses [15-21]. These systems allowed not only vital sign monitoring, but also posture recognition. However, later systems scaled back to only one or two sensors, concentrating on the respiration and 
cardiac parameters or on new sensing modalities [22-30]. Often, researchers who did use multiple sensors investigated which sensor provided the best results, intending to use only one of the sensors [31-33]. Chen et al. cited reducing computational complexity as a main driver towards a single signal [32]. Recently, multisensor systems have once again gained traction, generally with between 4 to 6 , but sometimes up to 30 sensors [34-42]. Kortelainen et al. originally embedded 160 sensors in a bed [40], but later reduced the number of sensors to just eight [43]. Cost was cited as the main reason for that reduction [40]. An exception to the use of fewer sensors is the application of sensor arrays to posture recognition. 8192 sensors have been embedded into a sheet to form high resolution pressure images [44], [45]. Fusion of the sensors for cardiopulmonary monitoring was not attempted with this number of sensors, but with appropriate fusion techniques, it would be possible.

Not only does signal fusion complicate ambient sensing, but in ambient sensing, one signal extraction block's interference signal is another's signal of interest. For instance, when extracting cardiac signals, breathing motion and movement artifact are sources of interference. However, these signals convey information that may be useful in their own right. In many applications, separating one signal from another can be done by analysis of stationary signal parameters; for example, the known signal frequencies can be amplified and undesirable frequencies suppressed. However, many ambient signals are non-stationary and non-deterministic, e.g., neither the exact shape of a breathing signal, its frequency (if periodic), nor its amplitude are known a priori. It is often the point of an ambient monitoring system to pick up on signals when they are most non-deterministic. For instance, it is disordered breathing, and perhaps not the regular rhythmic motion, that an ambient breathing monitor fundamentally seeks to uncover. Furthermore, while general frequency bands can be used to separate nondeterministic signals, overlapping spectral characteristics can inhibit such analysis. For example, movement artifact causes strong interference in both the breathing and 
cardiac bands.

\subsection{Objectives and Scope}

This thesis works towards providing dependable information from ambient sensors, where challenging conditions are present in the environment, the sensors, or the source signals themselves. Digital signal processing techniques are required that allow for the range of disordered source signals and interference that may be present. The main objective of this thesis is to develop signal fusion techniques that combine physiological signals recovered by multiple ambient sensors to produce a single accurate signal that can be used reliably in clinical applications. The light red "Source Signal Extraction" block shown in Fig. 1.1 is the main subject of this thesis. This block fuses the input signals from all of the sensors to produce an estimate $z^{\prime}\left(t_{n}\right)$ of the original source signal. From this estimate, information may be extracted.

Five concrete objectives emerge:

1. Identify conditions of fallibility for fusion algorithms extracting physiological signals from ambient sensors;

2. Develop new, more robust multisensor fusion methods;

3. Analyze new and existing algorithms, considering the identified conditions;

4. Identify new applications of clinical monitoring that would benefit from ambient sensing;

5. Apply ambient technology to the analysis of clinical data.

Particular emphasis is placed on the application of constructing breathing signals for respiratory pattern analysis from bed-based multisensor platforms. The "smart 


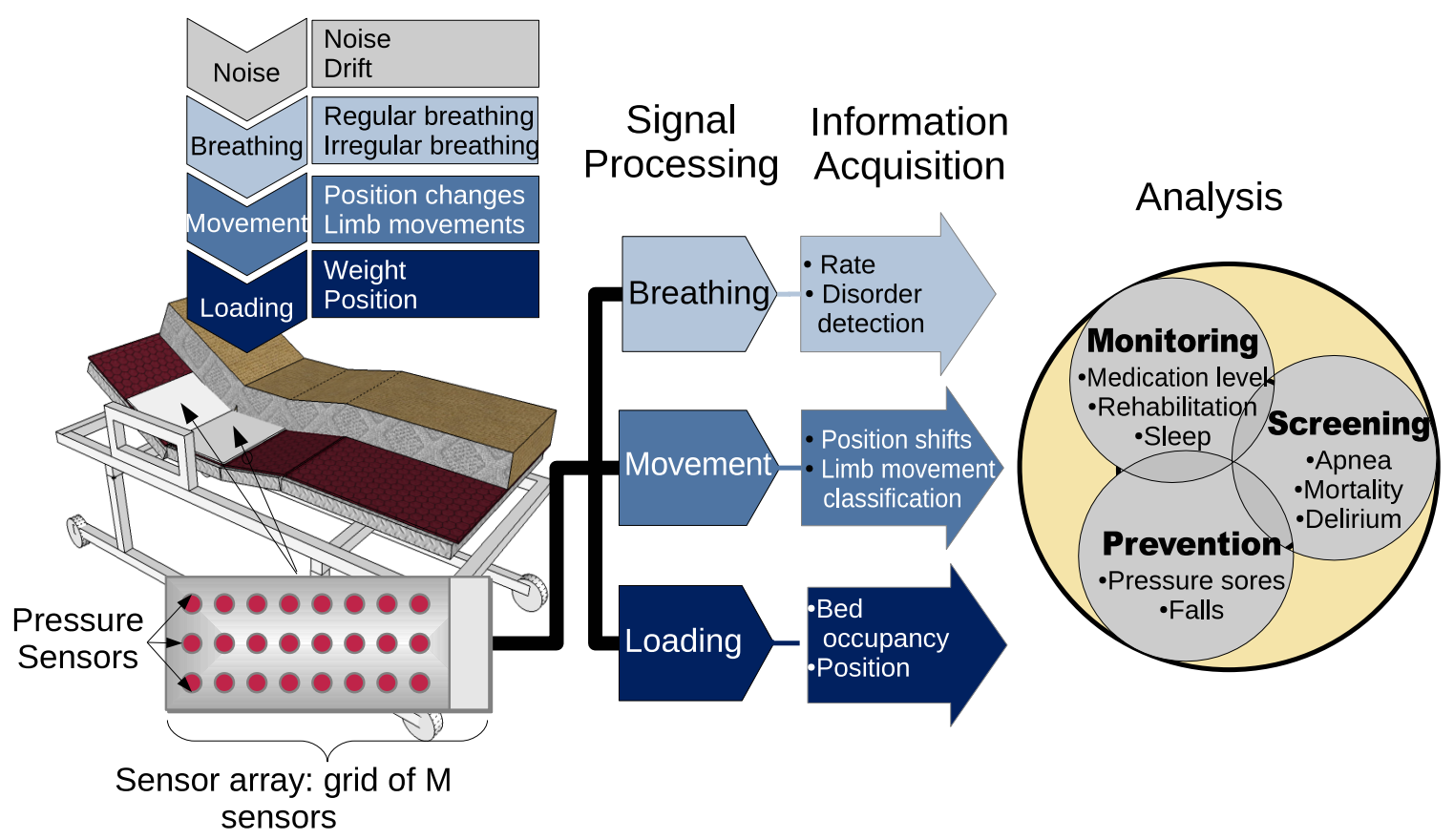

Figure 1.2: The sensorized bed can be used for prevention, screening, and monitoring

bed" concept provides unobtrusive monitoring of a bed occupant using pressure sensors. Fig. 1.2 shows this sensorized bed equipped with a pressure sensor array, as a component in an ambient system for prevention, screening, and monitoring. An array of pressure sensors below the mattress captures loading, movement, and breathing from the bed occupant with the intent to extract clinically useful information through appropriate processing. The middle stage of signal processing is the main topic for this thesis, with some consideration to information and analysis in the final contributory chapter.

This thesis examines the components of processing for signal fusion, including signal alignment by detecting polarity reversals that arise between sensors. Another component in signal alignment are correcting for delays between sensor signals. Breathing signal delay correction can be found within the scope of Daphne Townsend's Ph.D. thesis [46].

It is possible for ambient systems to pick up multiple source signals of the same 
type. For instance, two people sharing a sensorized bed produce two unique breathing signals and each would need to be separated from the other. However, the scope of this thesis is limited to a single bed occupant.

Although the thesis makes use of pressure sensors for experimental purposes, the developed methods are independent of the sensing modality. However, this modality does provide some limitation on achievable output. The usage of under-mattress pressure sensors allows extraction of breathing effort, but not an absolute respiratory volume. The scope of this thesis with respect to respiratory signal extraction and breath amplitudes is limited to relative breathing effort amplitudes. Analysis of breathing patterns is independent of absolute air volumes. Additional important aspects of ambient medical monitoring that reside outside the scope of this thesis include data transmission, storage, and security. Although challenges exist in each of these areas, they are not dealt with here.

\subsection{System Overview and Thesis Organization}

A fusion system designed to obtain an estimate of a real-world source signal unwraps the signal from the system response and reduces interference. From the model in Fig 1.1, the general signal architecture for ambient multisensor signal analysis is depicted in Fig. 1.3. Characterization mitigates the effect of the magnitude of the system response, alignment amends delays between signals and sign changes due to system response, and signal fusion joins the signals that were split across $K$ sensors.

This thesis is ordered in a bottom up approach, according to the flow shown in Fig. 1.3. However, before the signal extraction components are addressed, Chapter 2 reviews related research and pertinent open research questions. Chapter 3 then catalogues the system setup used for experimentation and details the experiments that were run to provide datasets for use throughout the thesis. The following four 


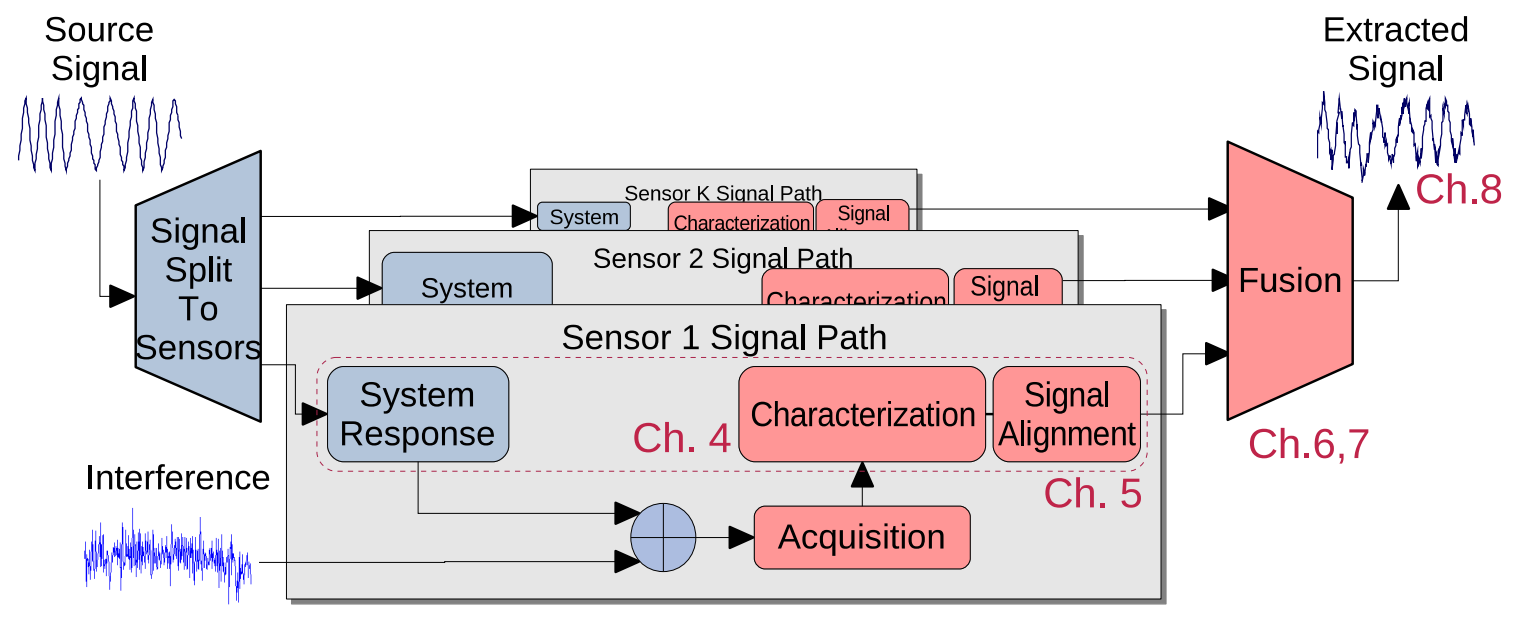

Figure 1.3: The system architecture for source signal extraction

chapters each deal with a component of source signal extraction labeled in Fig. 1.3. Chapter 4 examines characterizing and linearizing the system response. Chapter 5 studies methods to align signal by correcting reversals that flip signal polarity. Once the signals are aligned, fusion methods can be applied in Chapter 6. Chapter 7 considers enhancing fusion by employing an ensemble of fusion methods. Proposed techniques are applied to clinical data, collected from participants in a palliative care ward, in Chapter 8. Conclusions are provided in Chapter 9. An appendix provides background information about palliative care.

\subsection{Thesis Contributions}

The general contribution of this thesis is the development and optimization of algorithms to extract ambient breathing signals in suboptimal conditions. A number of specific contributions to the field of ambient physiological signal extraction were made for each of the stages of processing shown in Fig. 1.3.

The work completed for this thesis resulted in a number of publications with coauthors. For a complete detailing of the work involved and the division of labour, 
please see the Statement of Originality at the beginning of this thesis. The contributions listed here are all the original work of the author of this thesis.

\subsubsection{Contributions in Ambient System Response Characterization}

Contributions were made in the domain of sensor array measurement to quantify environmental response and sensor array response. Since many systems are tested in controlled laboratory settings, it is possible that use in real environments could be corrupted by dampened signals. Contributions were made to environmental response analysis for pressure sensor systems by investigating the impact of variable cushioning between the sensors and the individual to be monitored. Assessment was made of the power lost and the reduction in the number of sensors with available physiological signals. Power loss modeled according to mattress thickness showed that coil and foam mattresses attenuated breathing signals at a rate of $0.7 \mathrm{~dB} / \mathrm{cm}$ while a futon mattress attenuated them at $0.8 \mathrm{~dB} / \mathrm{cm}$. The study that considered these contributions is the first to quantify the impact of furnishings as a source of performance loss in ambient sensing. It also indicated the importance of a processing system that is robust to low signal to noise ratios and low signal availability when placing sensors beneath, rather than on top, of a mattress.

Not only is the environment responsible for the system response, but the sensor itself and the array configuration also factor in the response. Contributions were made to characterize the ambient sensor array through development of a theoretical model for Kinotex ${ }^{\mathrm{TM}}$ pressure sensors and linearization of signals using a neural network to take into account both non-uniform spatial response and non-linear sensor response. A further contribution was to introduce a shortened calibration protocol by training the neural network with modeled data. These methods allow a sensor array to 
provide estimation of applied force according to the location of the application. The contributions made to system response characterization are presented in Chapter 4, and published primarily in:

[47] M. Holtzman, A. Arcelus, I. Veledar, R. Goubran, H. Sveistrup, and P. Guitard. "Force estimation with a non-uniform pressure sensor array." In "Proc. 2008 IEEE Instrumentation and Measurement Technology Conf. (IMTC 2008)." pages 1974-1979 (2008).

[48] A. Arcelus, M. Holtzman, I. Veledar, R. Goubran, H. Sveistrup, and P. Guitard. "Contact location estimation from a nonlinear array of pressure sensors." In "Proc. 2008 IEEE Int. Conf. Instrumentation and Measurement Technology (IMTC 2008)," pages 1969-1973 (2008).

[49] M. Holtzman, D. Townsend, R. Goubran, and F. Knoefel. "Validation of pressure sensors for physiological monitoring in home environments." In "Proc. 2010 IEEE Int. Workshop Medical Measurements and Applications (MeMeA 2010)," pages 38-42 (2010).

Further, the application of this work is reflected in other publications, including:

[50] A. Arcelus, M. Holtzman, R. Goubran, H. Sveistrup, P. Guitard, and F. Knoefel. "Analysis of commode grab bar usage for the monitoring of older adults in the smart home environment." In "Proc. 2009 Ann. Int. Conf. IEEE Engineering in Medicine and Biology Society (EMBC 2009)," pages 6155-6158 (2009). 


\subsubsection{Contributions in Signal Alignment}

Signal alignment is an important pre-conditioning step for many fusion algorithms. A key stage of breathing signal alignment from bed-based sensors is correction of systemic signal polarity reversals. Contributions to signal alignment include developing and evaluating three methods of reversal detection including a correlation coefficient method, a method that assesses the sign of the slopes of the input signals, and an adaptive slope analysis technique. These last two fused rather than selected a reference signal.

Comparisons between methods were made using simulated signals with added drift, noise, and burst interference. These showed that the two slope-based methods were more robust to interference. Further, an assessment of fusing rather than selecting the reference demonstrated the impact of this fusion, particularly in AWGN noise, where up to $15 \%$ more sensor polarities were accurately assessed due to this fusion alone. Comparisons were also made using filtered and unfiltered experimental data. Lowpass filters were shown to be moderately effective at mitigating the effect of noise. The ultimate effect of applying new methods of reversal detection was to increase signal quality to acceptable levels in $8 \%$ more segments and improve respiratory rate estimates by $24 \%$, even in the relatively controlled laboratory conditions. For deployment in the less controlled home environment, the improvements offered by these algorithms could be even larger, leading to more reliable breathing signal monitoring. This work appears in Chapter 5. Related publications include:

[51] M. Holtzman, R. Goubran, and F. Knoefel, "Robust coherent sensor combining with decision directed reversal correction," IEEE Trans. Instrum. Meas., Submitted.

[52] M. Holtzman, A. Arcelus, R. Goubran, and F. Knoefel. "Breathing signal fusion in pressure sensor arrays." In "Proc. 2008 IEEE Int. Workshop Medical 
Measurements and Applications (MeMeA 2008)," pages 71-76 (2008).

[53] D. I. Townsend, M. Holtzman, R. Goubran, M. Frize, and F. Knoefel. "Measurement of torso movement with delay mapping using an unobtrusive pressure-sensor array." IEEE Trans. Instrum. Meas. 60(5), pages 1751-1760. (2011).

\subsubsection{Contributions in Data-Level Physiological Signal Fusion}

This work takes the novel step of describing many previously presented techniques of ambient breathing signal fusion as forms of linear diversity combining. The work presented herein marks the first time that maximal ratio combining has been applied to physiological signal fusion. This technique requires estimating signal and noise powers of the individual sensor signals, and three methods were advanced to perform this estimation. The first used a simple temporal measure of variance, while the second and third used spectrally-estimated signal and noise. The spectral estimation requires knowledge of the breathing frequency, assumed to be the frequency with the peak power in the respiratory band. The second method estimated the breathing frequency at each sensor individually. This individual estimation meant that sensors with interference reported a stronger signal than was warranted by selecting a frequency with a strong interference signal. To limit mistaking interference for signal, the third method pre-fuses the signal using equal gain combining, selects the breathing frequency using the fused signal, and then estimates the spectral signal and noise powers on the individual sensors.

Selection combining was also examined and a method of selecting the sensor using both signal to noise ratio and in-band signal power was presented. This selection method was used as a fusion method in its right and also applied to blind source 
separation. In blind source separation, multiple output components are often involved and selection combining was proposed to evaluate which output component held the breathing signal while rejecting components that held movement interference.

Comparisons were made between proposed and conventional methods of signal fusion in varying signal conditions by testing them with synthesized and real-world data. Eleven methods were tested by fusion of a breathing signal dataset with synthesized corruptions of added noise and drift interference, and datasets of movement artifact and disordered breathing. A final validation dataset was used to explore the methods in laboratory conditions. This was the same dataset that was used for analysis of reversal detection, which allowed evaluation across both stages of fusion. Fusion algorithms that do not require reversal detection can be superior to weighted summation methods (that require reversal detection) when the reversal detection stage was poorly executed. Maximal ratio combining and adaptive reversal detection weights both produced generally good fusion performance. A key finding was that, although these methods outperformed the others, they were not always the best technique, particularly when breathing signal availability was low. This work is found in Chapter 6 and resulted in these publications:

[54] M. Holtzman, R. Goubran, and F. Knoefel. "Maximal ratio combining for respiratory effort extraction from pressure sensor arrays." In "Proc. 2011 IEEE Int. Workshop Medical Measurements and Applications (MeMeA 2011)," pages 88-92 (2011).

[55] M. Holtzman, R. A. Goubran, and F. Knoefel. "Breathing sensor selection during movement." In "Proc. 2011 Ann. Int. Conf. IEEE Engineering in Medicine and Biology Society (EMBC 2011)," pages 381-384. Boston, MA (2011). 


\subsubsection{Contributions in Context-Aware Signal Fusion}

The discovery that some methods are sensitive to certain signal and environmental conditions led to the development of a system that could select the most appropriate fusion method for the conditions. An ensemble fusion method was instigated that fuses the signal with multiple fusion methods and then uses a trained classifier to assess the signal conditions and select the best method. Signal features were identified that could provide contextual clues regarding environmental and signal conditions and the system was implemented using a support vector machine (SVM). Candidate fusion methods were assessed and ultimately four of eleven methods were incorporated into the system. The SVM parameters were optimized and the best parameters were reported. Testing of the method was performed using a test set that was held back from the training set of corrupted and real-life signals. 55\% accuracy was achieved for selecting the best fusion method in degraded conditions, leading to a more robust fusion algorithm, with fewer undetectable breaths and a lower mean error. It was also tested on the validation dataset and the dataset used to evaluate multiple mattresses. The method was able to meet and exceed the fusion quality of the best method for each of these sets, even as the best method differed for each of the data sets. The details of this work are found in Chapter 7 and preliminary related work has been accepted for publication:

[56] M Holtzman and F Knoefel, "Ambient Context in Breathing Signal Fusion," in Proc. 2014 IEEE Int. Workshop Medical Measurements and Applications (MeMeA 2014), Portugal, Accepted.

\subsubsection{Contributions to Ambient Sensing in Clinical Care}

Two clinical fields that could benefit from ambient sensing were suggested in this work, cognitive medicine and palliative care. The specific parameters that could be 
extracted from a bed-based system to support clinical care in those fields were also detailed. Contributions were made to palliative care by validation of the technology for motion monitoring in an observer experiment, and by studying the respiratory parameters related to the end of life. Predictors of survival time based on breathing rates and breathing amplitude and interval irregularity were revealed. Tachypnea (fast breathing) was confirmed as indicative of approaching mortality, as all participants showed tachypnea in the week before death. These predictors could be used to enable early transfer of the patient to the home, better fulfilling their wishes for place of death. This is the first study of its kind to continuously monitor patients in a palliative care setting. These contributions are detailed in Chapter 8 and resulted in these publications:

[57] M. Holtzman, R. Goubran, F. Knoefel, and J. Pereira, "Measurement of respiratory changes in palliative care," in Proc. 2012 IEEE Instrumentation and Measurement Technology Conf. (IMTC 2012), pages 1472-1476 (2012).

M. Holtzman, F. Knoefel, J. Pereira, and R. Goubran, "Unobtrusive Monitoring of Respiratory Variability in Palliative Care: A Small Case Series," in Proc. Int. Congress on Palliative Care, 2012, vol. 28, pp. 219-219.

Related publications to enable clinical breathing analysis at home and supported the work for these contributions included:

[58] V. Joshi, M. Holtzman, A. Arcelus, R. Goubran, and F. Knoefel, "Highly survivable bed pressure mat remote patient monitoring system for mHealth," in Proc. 2012 Ann. Int. Conf. IEEE Engineering in Medicine and Biology Society (EMBC 2012), pages 268-271 (2012).

[59] D. Townsend, M. Holtzman, R. Goubran, M. Frize, and F. Knoefel. "Relative thresholding with under-mattress pressure sensors to detect central apnea." 
IEEE Trans. Instrum. Meas. 60(10), 3281-3289. (2011).

[60] D. Townsend, M. Holtzman, R. Goubran, M. Frize, and F. Knoefel. "Effect of windowing on central apnea detection." In "Proc. 2010 IEEE Int. Workshop Medical Measurements and Applications (MeMeA 2010)," pages 117-120. Ottawa, ON (2010).

[61] D. I. Townsend, M. Holtzman, R. Goubran, M. Frize, and F. Knoefel. "Simulated central apnea detection using the pressure variance." In "Proc. 2009 Ann. Int. Conf. IEEE Engineering in Medicine and Biology Society (EMBC 2009)," pages 3917-3920 (2009).

[62] A. Arcelus, M. H. Jones, R. Goubran, and F. Knoefel. "Integration of smart home technologies in a health monitoring system for the elderly." In "21st Int. Conf. Advanced Information Networking and Applications Workshops (AINAW 2007)," volume 2, pages 820-825. Niagara Falls, ON, Canada (2007). 


\section{Chapter 2}

\section{Background Review}

This chapter reviews previous research in the domain of ambient sensing that is relevant to this thesis work. The first section provides a characterization of the breathing signals that are the main focus of extraction. Factors involved in ambient system response are probed in Sec. 2.1, including sensor location and modality. With this in mind, the signal processing algorithms that have been applied to breathing signal extraction are scrutinized in Sec 2.2. An overview of clinical applications of bed-based ambient systems is provided in Sec. 2.3. Finally, the reviewed information is summarized in Sec. 2.4.

\section{$2.1 \quad$ Factors of System Response}

\subsubsection{Sensor Location}

Fig. 2.1 depicts the possible locations of bed-based ambient sensors. Bed-based sensors can be embedded in a specialized mattress [14,22,63-65], but separate sensing sheets or pads offer flexibility of placement and operation with existing furnishings. Such pads have been placed under the pillow $[21,32,66,67]$, on top of the mattress $[26,28,30,68-70]$, below the mattress $[16,17,23,24,31,71]$, below bed posts $[36,38$, 


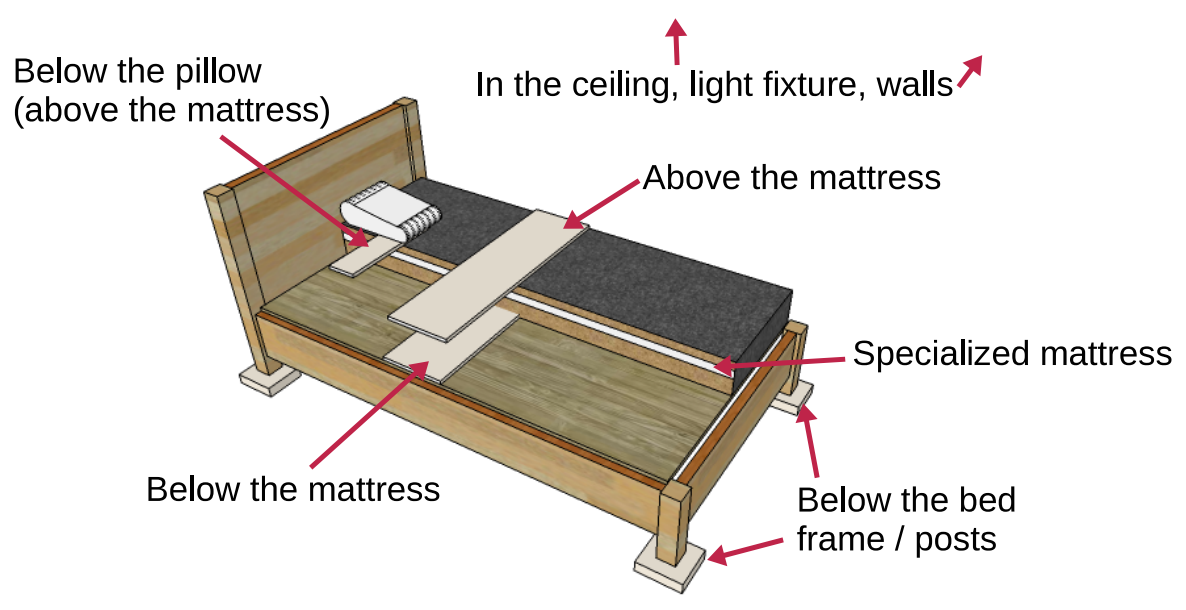

Figure 2.1: Possible locations of bed-based sensors

$39,41,42,72]$, and even embedded into the bed sheet above the body [73]. Instead of using the bed itself as the sensing platform, sensors have also been embedded into fixtures. Doppler systems and microphone sensors can be placed in a convenient spot in the environment surrounding the bed, such as in the ceiling light fixture [74].

An aspect considered for selecting a sensing location is visibility. For smart home technology to be accepted, it is important that the system is unobtrusive and private [75-77]. Smart home devices should not only be unobtrusive to users, but be "undetectable to the casual observer" [77]. Even a bed sensor on top of a mattress drew comments when it was unconcealed [76]. The optimal positioning for the desired physiological signal may also influence the sensing location. For instance, sensors may be placed near the expected chest and abdominal regions of the mattress for respiration analysis, or near the heart for cardiac measurement [26,28,30,68-70]. Strategic placement is especially important for single sensors or arrays that cover only a small area. Unless sensors are soft and conformable, they may be uncomfortable to sleep on above the mattress. Furthermore, routine sheet changes can disrupt sensors placed on top of a mattress or below pillows. Sensors placed below mattresses avoid these issues, but signals can be attenuated by the cushioning. 
Studying how this attenuation affects signal availability is relevant. There have been few explorations of how cushioning thicknesses and types affect signal attenuation. Four types of mattresses were considered by Watanabe and signal to noise ratios (SNR) were shown to be affected by the types [23]. Exploration of the factors that cause this variability would be useful.

Not only does the sensor location affect the signal, but the position of the bed occupant with respect to the sensor may also affect the signal. The effect of posture (e.g., supine, prone, lateral) [23,28] and sensor positioning [23,33] have been considered. An additional effect is signal reversal. In multisensor systems, a subset of sensor input signals can be reversed with respect to the rest of the inputs: some signals fall while others are rising and vice versa. Nishida et al. noted that reversals were common from their bed-based pressure sensors [15]. Breathing signals are established from small weight shifts, but while one sensor is loading, another is unloading. Reversals are either ignored by the system or must be detected. By ignoring them and absorbing the mismatch, system performance is degraded in applications that rely on signal coherency, such as weighted summation of sensor inputs. Methods of reversal detection are discussed in more detail in Sec. 2.2.3.

\subsubsection{Sensor Modality}

Physiological signals have been captured by sensors in the environment in a variety of ways, but most ambient sensing modalities fall broadly into five main categories: pressure systems, Doppler systems, audiovisual systems, thermal systems, and textile electrodes. Pressure systems are generally placed where an individual would exert pressure when they lie, sit, or stand, and have included many modalities of sensors including pneumatic sensors $[22,23,31,32,63,67]$, piezoelectric polymers $[24,30,33,73,78,79]$, load cells $[38,39,41,42,72]$, force sensitive resistors (FSR) [16-18,21,68,71], hydraulic 
sensors $[24,66,80]$, capacitive sensors $[14,69]$, indentation measurement potentiometers [65], electret films and foils [27,40], optical sensors [28,81], and strain gauges [82]. Doppler sensor modalities, including radio frequency transmitters/receivers [83-88] and ultrasound sensors $[63,89,90]$, are placed with a line of sight to the subject so that reflected waves capture bodily motion. Audiovisual systems use video [91-93], or microphones [74]. They are perhaps not as unobtrusive to users with privacy concerns as other modalities [75]. Thermal systems use temperature, either passively through thermistors in sheets or infrared sensors mounted under beds or in walls [19,94-97]; or actively by diffusing infrared light through a mattress and picking it up with a photo transistor [98]. An infrared camera system [99], is a crossover between an audiovisual system and a thermal system; the individual sensors are thermal in nature, but it is the complete "picture" that provides the motion monitoring. Textiles electrodes are woven into sensing strips or bed sheets $[26,100,101]$.

Although theoretical modeling of the sensor for linearization purposes is likely unique to the specific modality, the manifestation of the physiological signals through the sensors are often similar and thus the signal processing techniques involved for breathing signal extraction are comparable. Kortelainen et al. has noted that there were not any significant differences between using different pressure sensing modalities [43]. The following section reviews signal processing techniques and highlights areas of work.

\subsection{Ambient Sensor Signal Processing}

In Chapter 1, three stages of signal processing for extracting a source signal from ambient arrays were identified: conditioning, characterization, and fusion. Conditioning removes interference, usually by filtering. Methods of conditioning are usually targeted to specific methods of characterization and fusion. These last two are reviewed 
in Sec. 2.2.3 and Sec. 2.2.4, respectively.

\subsubsection{Breathing Effort Signals}

Breathing occurs in humans as both quiet, unconscious breathing, called eupnea, and forced or laboured breathing, called dyspnea [102]. Eupnea employs the muscles of the diaphragm and intercostals during expiration, with no active muscular contractions during expiration. On the other hand, dyspnea requires accessory inspiratory and expiratory muscles in addition to the intercostals and diaphragm, such as the abdominal muscles [102].

Given both of these two breathing types, breathing can be regular, with relatively stationary breathing amplitudes and intervals between breaths during eupnia; or nonstationary and non-deterministic in shape, amplitude, and interval during dyspnea. Generally, even during eupnea, there is non-stationary behaviour as central nervous system reflexes regulate the breathing cycle [103]. Furthermore, speech, sighs, coughs, and yawns may be interspersed in the breathing, and changes to rate and amplitude can occur suddenly, particularly where breathing is consciously changed. Signal processing that depends on stationary behaviour may work well enough with regular breathing, but may be challenged by irregular breathing.

\subsubsection{Signal Conditioning}

Input sensor signals can be conditioned to enhance the breathing components and suppress interference. A number of methods of conditioning are available, including filtering, wavelet transforms, and empirical mode decomposition.

Finite impulse response (FIR) and infinite impulse response (IIR) filtering have been used by researchers to reduce interference components outside of the spectral band of interest. FIR filters provide linear phase response, avoiding distortion caused 
by group delay [104]. A narrow respiratory band removes more interference than a wide one; however, restricting the band to be too narrow may mean missed breathing signals if the fundamental breathing frequency lies outside of the band.

Wavelets are useful for noise reduction in signals that exhibit non-stationary behaviour. Wavelets can be viewed as a type of low-pass filtering that separates a signal into low and high frequency components. By recursively repeating this separation on the low frequency component, a multiresolution wavelet decomposition is formed that a represents orthogonal frequency channels while retaining temporal information [105]. Some researchers have extracted the breathing signal using the output of a constant wavelet decomposition scale [73], [106]. However, Niizeki et al. commented that the waveforms produced were distorted. In that work, this was not a problem because their aim was "not at measuring accurate waveforms", but rather to measure breathing interval [73]. Using more than a single decomposition scale can produce a more accurate waveform and such a scheme has been used to reduce baseline drift [107]. However, movement artifact could influence the decision of which scales to include, ultimately reducing the accuracy of the output waveforms when movement was present [107].

Empirical mode decomposition (EMD) is another useful tool for nonlinear and non-stationary signals [108]. Similarly to wavelet analysis, EMD can be viewed as a multiresolution filter bank [109].

\subsubsection{Reversal Detection and Correction}

Detecting and correcting the reversals ultimately improves system performance.

When only two sensors are involved, they need only be compared to each other. When more than two sensors are involved, each sensor is compared to a reference signal. There also exist related bodies of work for phase reversal detection and correction in electroencepholography (EEG) focal area detection [110] and respiratory 
analysis [111]. Typically, one sensor is chosen from among the sensors to become the reference [15], [53]. However, the selection of a single reference sensor could be prone to error during interference. Just as signal fusion produces a more accurate signal, it may also produce a more accurate reference signal. Sensor fusion for reference signals has been applied by Wang et al. [112], although comparison of fusion to selection-based methods for reference signal generation has not been addressed.

Once a reference is chosen, the problem of reversal detection in sensor arrays is conventionally approached as a form of time delay estimation (TDE). Both Wang et al. [110] and Nishida et al. [15] used cross spectral phase analysis to compare each sensor to the reference, using the phase component at the frequency with the highest peak in the frequency band of interest. Wang et al. worked with 1024-point fast Fourier transformed (FFT) data from 5 second segments of EEG signals sampled at $200 \mathrm{~Hz}$, providing a resolution of $0.2 \mathrm{~Hz}$ [110]. Absolute phase differences greater than 100 degrees between the reference and sensor output indicated a reversal [110]. Nishida et al. worked with pressure sensors for breathing fusion. They did not detail the spectral transform, but did indicate that sensors with phase differences of $0.75 \pi$ to $1.25 \pi$ were considered as reversed, while those with phase differences of $-0.25 \pi$ to $0.25 \pi$ were considered as in phase and all other sensors were disregarded [15]. The spectrum of ambient sensors with strong breathing contains a large peak at the breathing frequency within the $0.1 \mathrm{~Hz}$ to $0.8 \mathrm{~Hz}$ range, with smaller peaks at harmonics of that frequency, and a possible peak at the heart beat frequency. However, for signals that have non-stationary periods, the breath peak may be smeared or there may be multiple peaks. When hampered by low SNR and spectral leakage from interfering signals, such as drift, the breath peak may be indiscernible. 


\subsubsection{Breathing Signal Fusion}

When multiple sensors provide a breathing signal, there is a need to combine the inputs from these sensors to produce a single representation of an individual's breathing.

\section{Homogeneous and Heterogeneous Data}

Combining data that comes from the same sensor is a homogeneous fusion, while combining data from different types of sensors or sources of information is heterogeneous data fusion. This thesis is primarily concerned with homogeneous fusion from one sensor modality.

\section{Fusion levels}

When the goal is to produce information from a group of signals, fusion can occur at a variety of points in the process: data-level, feature-level, and/or decision-level [113]. Data-level fuses the signals to produce a single time-series representation, feature-level extracts features from each signal and then fuses the features, and decision-level makes decisions based on each sensor signal and features extracted from each signal and then fuses the decisions. Often, if only a single specific aspect of the breathing signal, such as the respiratory rate or apnea detection, is desired, feature-level fusion may be appropriate. For example, Beattie et al. detected disordered breathing (apneas and hypopneas) from six load cells placed below the legs of a six-legged bed by extracting a set of features from each of the load cell inputs and combining all of these extracted features using a Bayesian classifier $[34,42]$. Respiratory rates may also be extracted in the same manner. For example, Mack et al. extracted respiratory rate from two sensing strips placed horizontally across a bed by calculating the rates from each strip and then averaging the results to produce a single respiratory rate [70]. Another method of feature-level respiratory rate fusion was introduced by the author of this 
thesis, using clustering and a weighted voting mechanism to fuse the respiration rates [52]. When more than one aspect of the signal is desired, feature-level fusion would result in fusion being performed for each desired aspect, unnecessarily increasing the complexity of the system.

Decision-level fusion would wait until a decision needs to be made to fuse the results. For example, in diagnosing sleep disturbed breathing, an apnea-hypopnea index (AHI) larger than a certain threshold provides a decision point for diagnosis. A decision-level fusion system would detect apneas at each sensor individually and also determine for each sensor if the AHI surpasses the threshold. Fusion would then be made on the decisions output from the individual sensors. This type of fusion was not encountered in reviewing work with bed-based analysis.

Although the breathing signal may be available from the sensors individually for feature-level fusion, data-level (or signal-level) signal fusion generally improves measurement accuracy [6]. For this second type of fusion, conditioned signals are combined to create a single respiration signal from which to evaluate respiratory information. When more than one respiratory parameter is desired, it may be more efficient to use a single signal rather than perform fusion operations for every desired parameter.

Data-level fusion methods in ambient systems have used the linear combining paradigm. Fig. 2.2 presents linear combining applied to data-level breathing signal fusion. In this method, sensor signals are conditioned by preprocessing, aligned, multiplied by a gain factor and then summed. Depending on the combining method, reversal correction is not always necessary. Gains are assumed constant over a short time period during which the signal itself is assumed to be relatively stationary. Signal fusion of breathing effort at the data-level can be broadly grouped into three categories of linear combining: sensor selection, weighted summation, and blind source separation. Linear combining is also used in communication systems for combining 


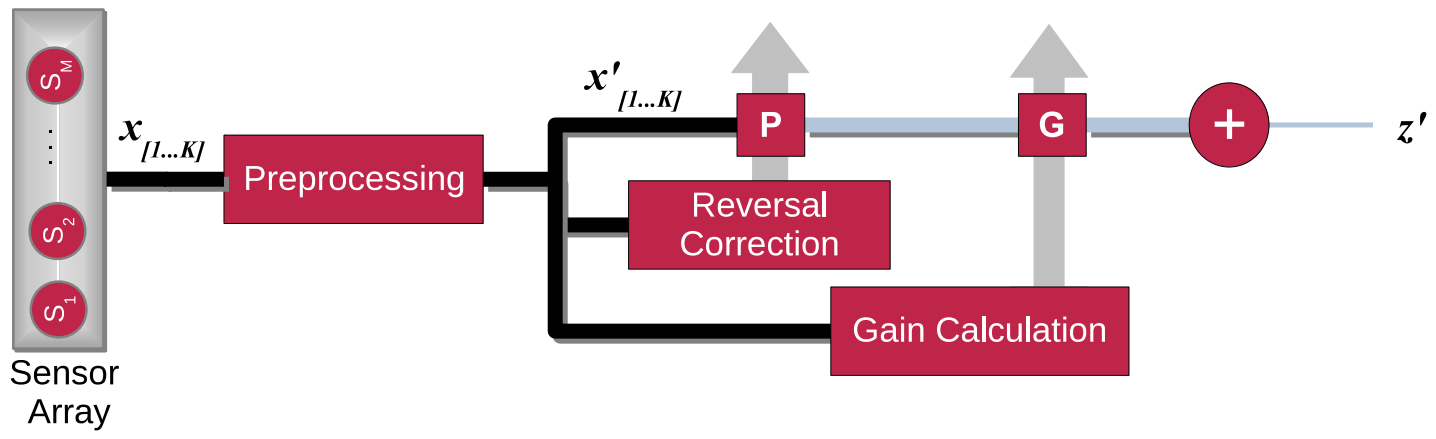

Figure 2.2: Block diagram of respiratory fusion as linear diversity combining

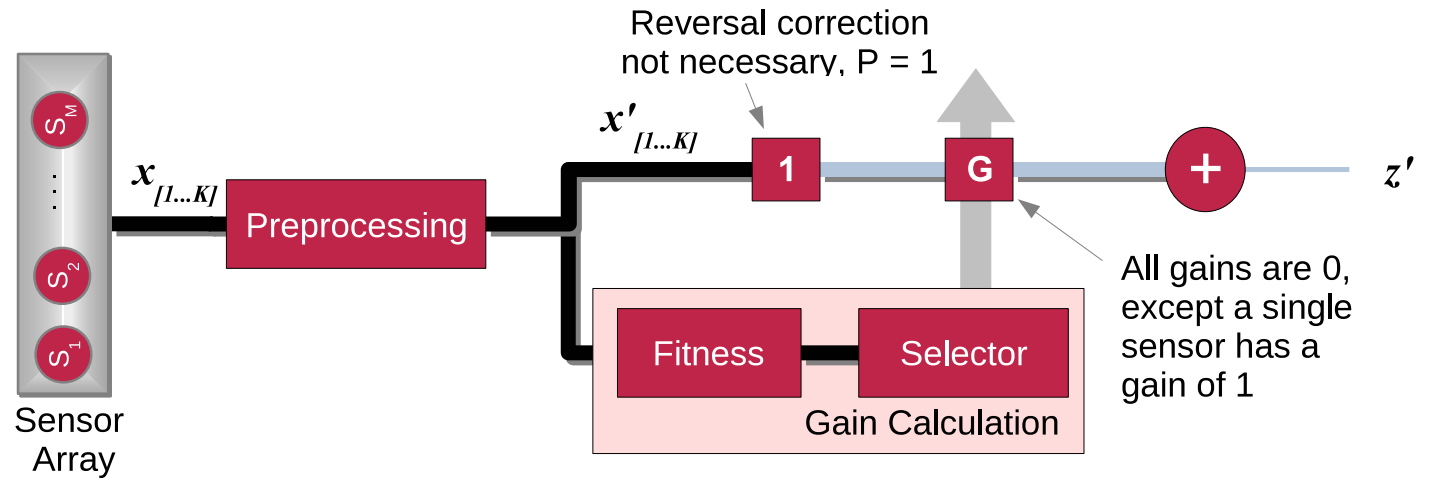

Figure 2.3: Block diagram of sensor selection as selection combining

signals received over multiple channels and termed linear diversity combining. A classic treatment of linear diversity combining by Brennan describes sensor selection and weighted summation methods [114], [115].

\section{Sensor Selection Combining}

Sensor selection combining represents the breathing effort signal using the input of a single sensor, chosen from amongst all of the sensors. Fig. 2.3 shows sensor selection as a linear combining method. For selection combining, all channels are weighted by 0 except for the single channel that has been selected, with a weight of 1 . Phase alignment correction is not necessary since no destructive interference will occur during summation. Let the selected sensor be denoted $k_{s}$ and the individual fitness scores 
be denoted $f_{k}$ :

$$
k_{s}=\arg \max _{k} f_{k}
$$

and

$$
g_{k}=\left\{\begin{array}{cc}
1 & \text { if } k=k_{s} \\
0 & \text { otherwise }
\end{array}\right.
$$

Spatial, temporal, and spectral methods of selection have been used. Sensor selection also encompasses systems where the selected sensor is predetermined and inputs from other sensors are ignored for the purposes of cardiopulmonary monitoring (although they may be used for other purposes), as in [69], [66], and [80].

To select a sensor spatially, Kimura et al. [20] classified the posture of the bed occupant first and then selected the sensor that was closest to the heart according to estimates of chest and head positions. However, posture classification before selection may be more complex than is warranted and errors in classification would impact the breathing fusion. Temporal methods analyze the fitness of the signals themselves by examining time-domain characteristics such as the minimum kurtosis [68], maximum signal amplitude [82] or maximum autocorrelation [116]. Autocorrelation makes use of the rhythmicity of the signal, which can also be inferred from the frequency spectrum. Niizeki et al. [73] and Vanderloos et al. [19] both selected the sensor with the highest total power in the respiratory frequency band, measured using the power spectral density (PSD) . Signals with strong breathing signals will include a peak in the PSD which will increase the power in that band.

Although there exists many methods of selecting an appropriate sensor for the purposes of breathing signal extraction, studies comparing the performance of the methods have not been made. The methods generally chose one feature for the selection criteria; hybrids of multiple features have not been examined. Furthermore, the signal spectrum could provide more information towards selection than just the 


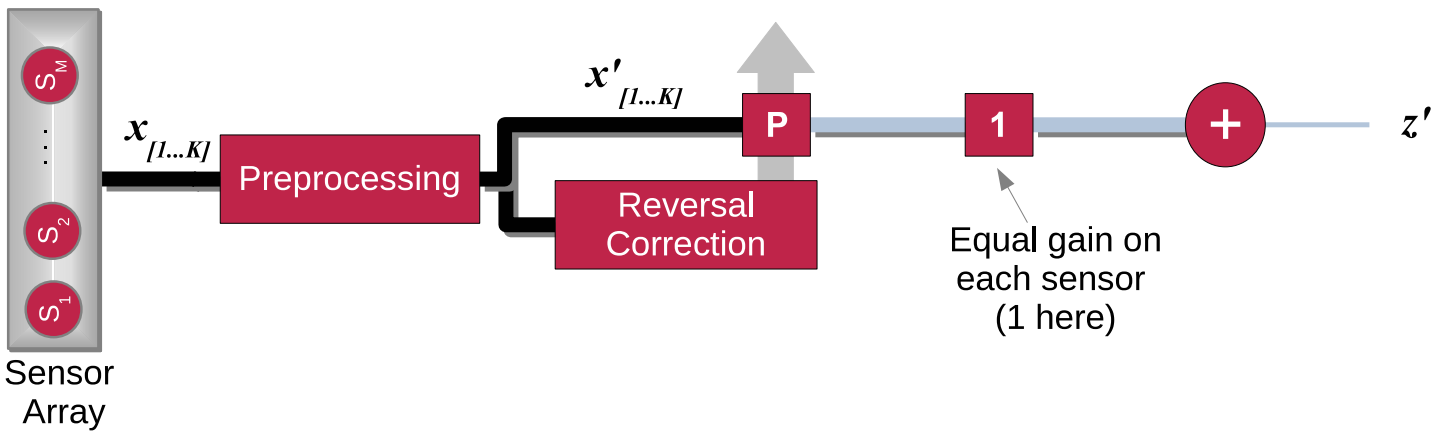

Figure 2.4: Block diagram of sensor summation as equal gain combining

total power in the respiratory band.

\section{Weighted Sensor Summation}

Instead of selecting one sensor, a breathing signal can be formed from the inputs of multiple sensors using a weighted sum of the sensor signals. Summation with equal weights, either using the sum or an average, is a simple method of fusion called equal gain combining (EGC) and is depicted in Fig. 2.4. For an EGC combiner, the gains are all set to the same value over all channels, usually 1 . In traditional communication systems, if all $K$ sensors have the same average SNR, $\gamma_{i n}$, then a $K$ sensor system increases SNR by a factor $1+(K-1) r^{2}$ over a single sensor system, where $\mathrm{r}$ is a dimensionless constant $0<r<1$ and is related to the distribution of SNR [114].

Straight summation has been performed in $[15,17]$. The sum of the squared differences [18] and the sum of absolute differences [21] avoid reversal correction, but provide two peaks per breath. Even without reversal correction, simple summation or averaging, may return an adequate breathing signal in some conditions [35].

By more intelligently weighting the sensors, it may be possible to emphasize sensors with strong breathing signals while de-emphasizing those without. Brink et al. [72] calculated weights using the centre of mass along the longitudinal axis of the bed. For a person lying straight along the bed on their back or front, this would 


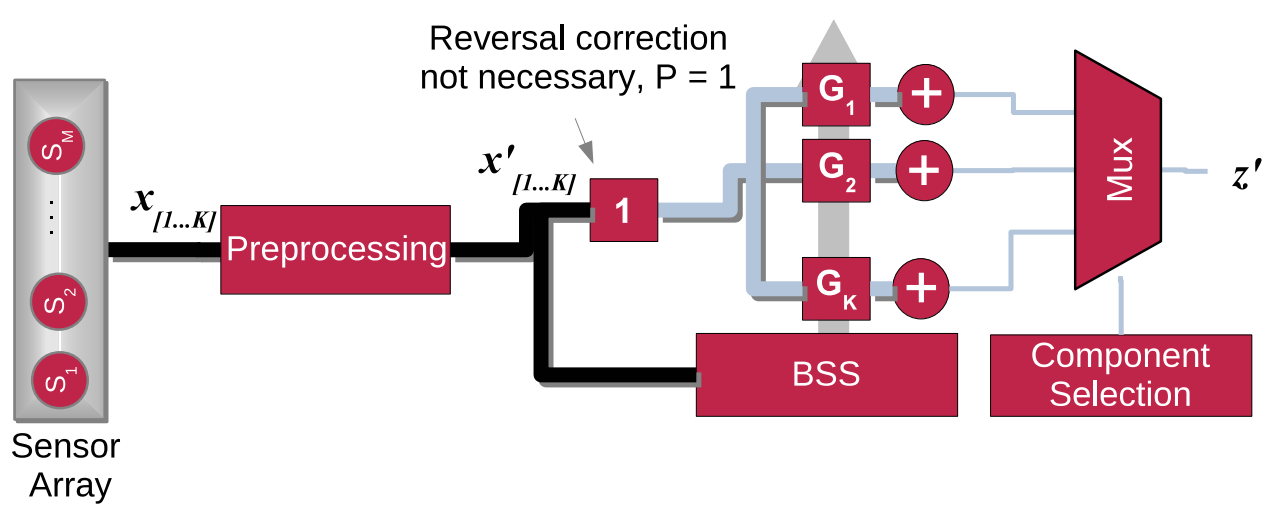

Figure 2.5: Block diagram of blind source separation as linear combining

pick up most of the weight shifts that occur during breathing, since breathing motion would not be expected to produce much side to side motion. However, an individual could lay diagonally on the bed, or bent in c-shape on his or her side, in which case there could be a significant lateral component that would be lost.

An important type of linear diversity combining that has not been applied to breathing signal fusion is maximal ratio combining (MRC). This method seeks to improve the SNR of the output signal by taking signal and noise power factors into account in the gain calculation. Without a priori knowledge of signal characteristics, it may be difficult to judge signal and noise power. How, and if, this method is applicable to ambient physiological sensing remains to be investigated.

\section{Blind Source Separation}

An approach to finding a more favourable weighting scheme that is indifferent to the person's position and posture is blind source separation (BSS). BSS uses the statistical analysis to separate signal sources and provides $K$ components for $K$ input signals. Fig. 2.5. Two varieties of BSS have been used for ambient multisensor breathing signal extraction: principal component analysis (PCA) and independent components analysis (ICA). 
Kortelainen et al. [43] used singular value decomposition (SVD), a type of PCA, to blindly separate breathing motion from interference using 160 sensors. SVD returns the eigenvectors of the covariance matrix of input sensor signals to find the principal axis of signal variation. For $M$ sensor signals, up to $M$ sets of eigenvector coefficients can be returned. The original signals multiplied by the coefficient weights form $M$ output signals that are linearly uncorrelated. Components are generally ordered from strongest to lowest, so that the component with the strongest variance (or eigenvector with the largest eigenvalue) is returned first. Breathing motion is the main cause of motion while a person is still, so the first component usually contains this signal. Since movement becomes the first component, the procedure was iterated with movement detection and removal when principal components changed within the epoch. There is complexity in detecting these changes that was not specifically detailed in [43], such as choice of window length and detection methodology. Furthermore, iteration may not be required, since the breathing may in fact already be available in a secondary or tertiary component of the PCA decomposition. This approach has not yet been considered.

Uchida et al. performed blind source separation using ICA to extract the respiratory signal from two sensors [67]. ICA assumes each sensor output is formed by a linear combination of multiple statistically independent and non-Gaussian sources and separates signals by finding mixtures that minimize the normality of their joint marginal distributions [117-119]. Independent component analysis outputs as many sources as sensors [118]. Uchida et al. expected three sources in the signals, namely noise, breathing, and the BCG, which would make the system underdetermined since there was not enough sensors for the number of sources [67]. They theorized that the BCG and the breathing would occupy one component and the noise the other, and used a lowpass filtering operation to separate the BCG from the breathing.

If ICA is incorporated in a multisensor signal with more than two or three sensors, 
the problem becomes overdetermined. Consideration of higher numbers of sensors and the selection of the output component should be investigated. Since ICA does not provide any ordering to the output sources, selection of the breathing signal from the two components is required, but this problem was not addressed by Uchida et al.

\section{Non-Linear Fusion Methods}

Linear combining methods are investigated in this thesis, but nonlinear techniques may also offer suitable fusion capability. Non-linear combining techniques usually transform the signal non-linearly, do linear combining on the transformed signals, and then inverse transform the result. For example, combining the intrinsic mode functions of an empirical mode decomposition has been performed to fuse breathing signals from ambient sensors [78]. Wavelets have been successfully used for multisensor image fusion [120-123] and similar strategies could be tested.

For either wavelet transforms or EMD to work well, without undue signal distortion, methods to select multiple decomposition levels (e.g. scales for wavelets, and intrinsic mode functions for EMD) for final combining are required. A single decomposition level may not contain the breathing signal throughout a segment, particularly when the breathing rate changes, switching the breathing signal from one decomposition level to another partway through the segment. Dai et al. selected decomposition levels based on spectral analysis [78], but spectral analysis is best used with stationary signals. To exploit the suitability of EMD to non-stationary signals, selection methods that do not rely on stationary signals would be useful.

\subsubsection{Coping with Movement}

Movement is the source of interference that is most often cited for causing problems for breathing analysis $[22,33,70,80]$. Movement artifact is a common occurrence in bed based systems, with between $3 \%$ and $32.5 \%$ of the night reported to be unavailable 


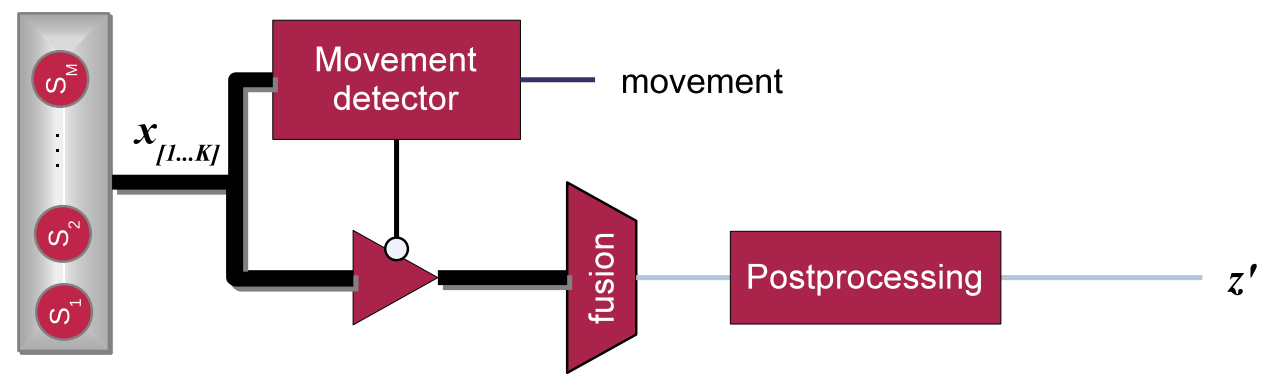

Figure 2.6: Conventional breathing analysis architecture

for monitoring due to movement $[22,23,43,66]$. Kortelainen et al. noted that it "varied strongly between subjects" [43] and most results show large inter-subject and intra-subject ranges of nocturnal movement.

Conventional breathing analysis architecture prioritizes movement detection over breathing extraction, to squelch breathing fusion and analysis during movements. Fig. 2.6 displays this architecture. Movement can be detected using preset thresholds [73], [68], but such thresholds must be high enough to allow for strong breathing signals when transmission to the sensors is strong and low enough to capture movements when transmission is weak. It is possible to capture the largest movements this way, but sensitivity to limb movements will either be low or the system will misconstrue strong breathing signals as movement. To remain sensitive to movement irrespective of signal amplitude, movement detectors can use statistical change detection to look for changes in the sample value distribution that are out of line with previous segments of data. Control limits and the generalized likelihood ratio (GLR) are both statistical tests for change detection that have been used for bed-based movement recognition $[38,80,81,124]$. principal component analysis can also be used to differentiate movements from breaths since breaths consistently occur along the same principal axis, while movements differ [43].

Statistical detectors tend to have false positive detections when sudden changes in breathing depth occur, such as breaths after apnea or single large sighs. For infrequent 
apneas or sighs, the loss of a few seconds of breathing data from a false detection could perhaps be tolerated. principal component analysis movement analysis may be more tolerant to sudden large breaths, but could also be similarly affected during apneas if the principal axis of breathing motion is lost. For subjects with frequent apnea episodes or breathing depth changes, both of these detector types may excessively discard breathing samples.

While movement artifact can be detected and rejected, in practice some interference is missed, particularly movements of low amplitude. Additionally, onset and offset detection mistiming may allow larger artifact to remain [38], [124]. To makes allowance for onset and offset detection mistiming, short periods before and after detected movements can also be discarded from analysis. For instance, Zhu et al. [80] halted breathing signal analysis not just during detected movement, but also 2.5 seconds before and after the detected movement. Mack et al. ignored full epochs if any movement occurred during the epoch [70]. Both of these approaches are effective at reducing mistiming problems, but worsen the problem of data loss due to false detections. Furthermore, Mack et al. found that many epochs with apnea were discarded due to the movement from arousals after apneas. To reduce false detections, sensitivity can be decreased, but this would result in further movement artifact in the breathing signals.

Assuming that there is undetected movement artifact that remains in a signal, it is important that a fusion process remain unaffected by this movement during the uncorrupted portion of a breathing segment. It would be useful to examine the impact of movement remaining in the breathing signal during fusion. 


\subsection{Clinical Applications of Bed-Based Sensing}

There are relatively few clinical studies of bed-based ambient systems, with most research still focussing on laboratory studies with healthy participants. Reviewed here are the clinical studies that have been executed and their field of clinical application.

The most obvious application for bed-based sensing is in the analysis of sleep, and most bed-based systems are aimed towards augmenting in-laboratory polysomnography or screening individuals for polysomnography from the comfort of their own beds. Such research is both relevant and useful. Sleep disorders are a major complaint of middle-aged and older adults, affecting a steady $20 \%$ of the population in all age cohorts over 45 years old [125].

Clinical experiments have investigated sleep architecture by extracting sleep timing parameters such as bed time and rise time [39,95], classifying sleep/wake segments $[37,126]$, and sleep staging of random eye movement (REM) versus non-REM (NREM) sleep $[31,40,127,128]$. Sleep disordered breathing has been identified through detecting obstructive apnea, hypopneas, and central apneas. [34, 42, 129, 130]. Finally periodic leg movements have been detected for screening of restless leg syndrome $[38,131,132]$. While many clinical experiments have been done with sleep, they have been all performed in a laboratory setting and have yet to proceed into the home environment.

Bed-based systems have also been used for rehabilitation. Sit-to-stand transfers of patients following a hip fracture or stroke have been analyzed [133,134]. Patients have also been monitored after a heart attack to see that they are getting proper bed rest and evaluate the progression of their movements in bed [96].

There are likely many medical fields that could benefit from ambient monitoring. Given that only two clinical domains of sleep medicine and rehabilitation have been trialed so far, the clinical applications of bed-based ambient systems have yet to 
scratch the surface of their potential.

\subsection{Summary}

Ambient sensors have been put in a variety of locations on, under, and around beds. How the environmental conditions dictated by these locations, impact the quality of signal extraction and the feasibility of obtaining the signal has not yet been addressed.

Few comparisons between methods for fusing ambient signals have been made. Components of the signal processing architecture could be isolated to determine how ambient conditions affect the processing stages. Signal alignment is a pre-conditioning step for a number of linear combining methods. In bed-based systems, correcting polarity reversals between sensor signals is the most important type of signal alignment, but has never been separately analyzed. Whether reversal detection could be improved by directly examining the rising and falling of the signals, rather than by oblique temporal and spectral methods of time delay analysis, is ripe for research.

Three categories of fusion methods were identified: selection combining, weighted summation, and blind source separation. There are open questions for each of these methods.

Selection combining: Can selection performance be improved by using more spectral information?

Weighted summation: Can MRC be applied to the linear combining of ambient signals, without a priori knowledge of signal characteristics?

Blind source separation Can ICA be applied when using more than two sensors, and how can the correct output component be selected in ICA or PCA?

Furthermore, comparing these methods to see how ambient conditions affect their efficacy has yet to be explored. 
Bed-based ambient systems have understandably focused on sleep monitoring, but other fields of medicine may also benefit from their application. In clinical care and home settings, ambient signal processing must be robust to irregular breathing and interference, and particularly motion.

The rest of this thesis explores these open research questions. Chapter 3 sets up the experiments and simulations that provide data for analysis in subsequent chapters. Chapter 4 explores environmental and sensor characterization, Chapter 5 focuses on reversal detection, Chapter 6 addresses signal fusion, and Chapter 7 considers adding context awareness to signal fusion. Applications of the bed-based sensing system are considered in Chapter 8, where the methods developed in the preceding chapters are then applied to clinical data. 


\section{Chapter 3}

\section{Experimental Setup and Data Collection}

This chapter details the technology and general setup for data collection. It also

develops a body of data for use in testing breathing signal extraction algorithms presented in later chapters. Two experiments with real participants were performed and detailed here. From the collected data, new data sets were created for specific scenarios of use. Basic usage sets included sets with clean respiration and posture information, sets with noisy respiration signals, and sets with a reduced number of sensors. Sets for testing irregular breathing and movement scenarios were also developed. Finally, the performance measures that are used in subsequent chapters for the evaluation of extracted respiratory signal quality are described.

\subsection{Sensor Technology}

\subsubsection{Pressure Sensor Mats}

The pressure sensor arrays used in all experiments were S4 Sensors Inc.'s (Victoria, B.C.) "Bed Occupancy Sensors" (BOS). These arrays contain 24 pressure sensors in a $90 \mathrm{~cm}$ by $24 \mathrm{~cm}$ package that is less than $1 \mathrm{~cm}$ thick. The actual sensorized area is $82 \mathrm{~cm}$ x $24 \mathrm{~cm}$; the remaining $8 \mathrm{~cm} \times 24 \mathrm{~cm}$ strip contains the electronics for analog 
to digital conversion, sampling, and transmitting the sensor data. Fig. 3.1 depicts the sensor mat.

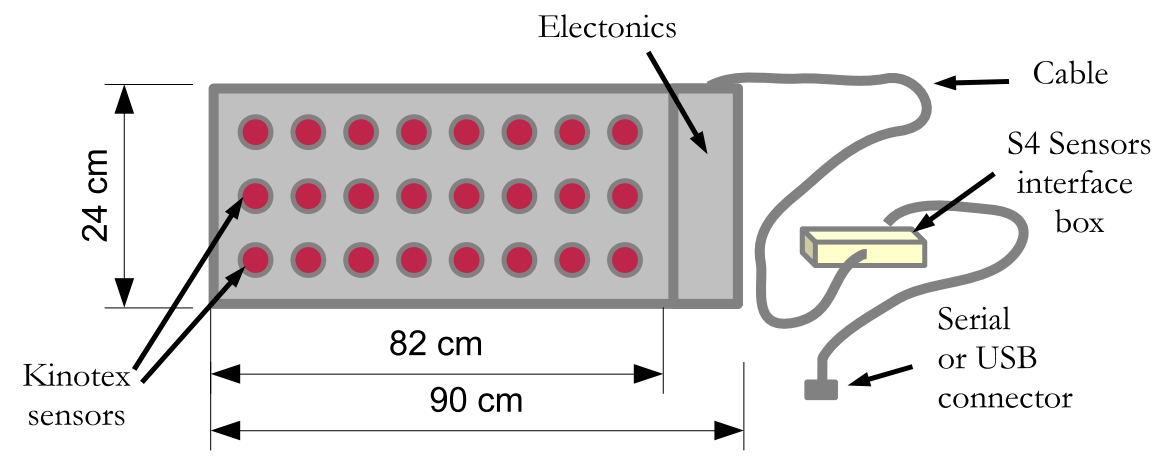

Figure 3.1: S4 Sensors BOS mat

\section{Kinotex ${ }^{\mathrm{TM}}$ Pressure Sensors}

Kinotex ${ }^{\mathrm{TM}}$ sensors populate the $\mathrm{S} 4$ Sensor's pressure sensor array. Their use in medical literature includes evaluation of body pressure towards pressure ulcer prevention [135], and to determine optimal postural support of a person in a wheelchair [136]. The BOS $^{\mathrm{TM}}$ sensor mat is comprised of 24 Kinotex $^{\mathrm{TM}}$ sensors. Kinotex ${ }^{\mathrm{TM}}$ pressure sensors are subject to the same problems as other pressure sensors: non-linearity, hysteresis, temperature dependence, and time-variance [137]. For example, one study recorded $0.9 \%$ creep during the third minute of a trial, and $30 \%$ hysteresis [138]

\subsubsection{Braebon Respibands}

The Braebon respiband system is made up of two respiratory inductance plethysmography (RIP) sensor belts, worn around the abdomen and the chest, and the Q-RIP interface box. RIP belts outputs are linearly proportional to the cross section of the thorax inside the loop [139]. The leads from the two belts plug into the Q-RIP interface box which provides filtering and amplification of the signals, providing a 0 to 1 $\mathrm{V}$ output with a mean of $0.5 \mathrm{~V}$. 
To sample these bands, the Braebon outputs were connected to a LabJack Corp. (Lakewood, CO, USA) U12 data acquisition device for analog to digital conversion and sampling. Data samples were input to a laptop from the LabJack device through a universal serial bus (USB). Fig. 3.2 presents a pictorial of the components in the respiband acquisition system.

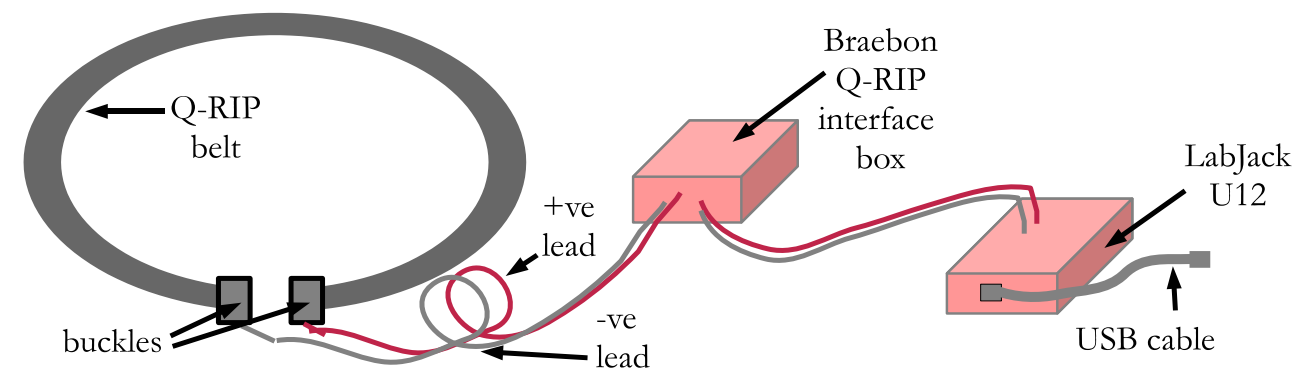

Figure 3.2: Respiband setup: band, interface box, and data acquisition board

\subsection{Data Acquisition and Analysis Software}

The software required during data acquisition and analysis included commercial software and software written by the author. The main platform for data conversion and analysis of recorded data was MATLAB (MathWorks), Version 7.8.0.347 (R2009a).

\subsubsection{Acquisition and Data Conversion}

Input from the sensors through their respective interface boxes were through serial or USB ports and controlled by commercial software specific to the interface box. All data acquisition software ran on the Microsoft Windows operating system (NT and $\mathrm{XP})$.

S4 Sensors Inc's proprietary software controlled the pressure sensors and collected the 11-bit, $10 \mathrm{~Hz}$ sampled data from the COM ports. The samples from all of the sensors on a single sensor mat were recorded by this software to comma separated 
values (CSV) files that included a timestamp for every sample. DAQFactory Express (Azeotech Inc., Ashland, OR, USA) software configured and collected Braebon respiband data to CSV files from the LabJack interface box, also with timestamps. The bands were configured to be sampled at $20 \mathrm{~Hz}$ (Multi-mattress experiment) and $100 \mathrm{~Hz}$ (Multi-participant experiment).

Software was written by the author in C to capture annotations during experiments. The software saved each annotation, along with a timestamp, to a CSV file. After data acquisition, the various CSV files from sensors and annotations were aligned according to the timestamps. The respiband data were resampled to match the sample times from the sensor mat as closely as possible. Pressure sensor data from multiple mats were merged, and the merged files were converted to binary files in MATLAB format.

\subsection{Experimental Data}

Two experiments were run to collect data for testing with the methods throughout the thesis. These two experiments are described here. Other experiments were pertinent to a single chapter and are described in the relevant chapter.

\subsubsection{Ethics Approval}

Ethics approval for all experiments in this work is covered by three ethics clearances, under two separate research ethics boards (REB). The first is with the Carleton University Research Ethics Board and the second is Bruyère Continuing Care Research Ethics Board. At Carleton, approval was granted for an application entitled "Presence, weight, and activity monitoring using a pressure pad". This approval covers the first two experiments, which were conducted on campus in the DSP research laboratory. It also provides for secondary data analysis of data collected under the 


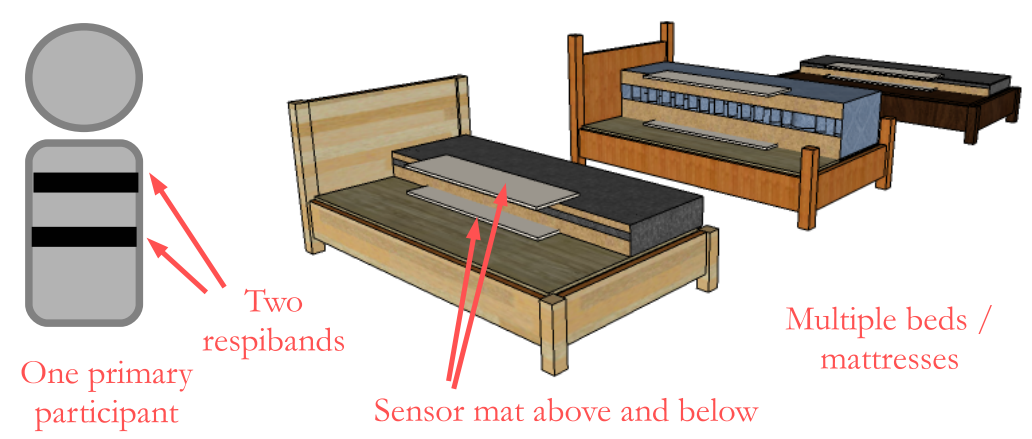

Figure 3.3: Components in the multi-mattress experiment

Bruyère Continuing Care REB clearance. The Bruyère Continuing Care Research Ethics Board, granted approval to two applications, entitled "Pilot Study Bed Sensor Array: Can it Provide Clinically Relevant Data in Palliative Care?" and "The Use of Pressure-Sensitive Mat Technology to Monitor Palliative Care Patients." These two approvals cover data recorded at Elisabeth Bruyère Hospital's Palliative Care Ward for experiments run in Chapter 8.

\subsubsection{Multi-mattress Experiment}

This experiment was aimed at comparing the effect of mattress type and thickness on output and validating algorithms across mattress types. This experiment was co-investigated with Daphne Townsend.

Fig. 3.3 depicts the multi-mattress experiment and the three categories of mattresses tested: foam, coil, and futon. Nine mattresses were tested with thicknesses ranging from $15 \mathrm{~cm}-35 \mathrm{~cm}$. Table 3.1 details these mattresses. Two pressure sensor mats were placed in the bed. One was placed above the mattress and the second was placed underneath the mattress, directly below the first. They were placed in a longitudinal orientation and centered below the pillow.

A single primary healthy control participant, a female researcher, wore two 
Table 3.1: Multi-mattress experiment: Mattress types and thicknesses

\begin{tabular}{|l|r|c|c|c||c|}
\hline & Description & Type & $\begin{array}{c}\text { Mattress } \\
(\mathrm{cm})\end{array}$ & $\begin{array}{c}\text { Pillow-top } \\
(\mathrm{cm})\end{array}$ & $\begin{array}{c}\text { Total } \\
(\mathrm{cm})\end{array}$ \\
\hline \hline 1 & deluxe & coil & 23.0 & 12.0 & 35.0 \\
\hline 2 & superior & coil & 18.0 & 15.0 & 33.0 \\
\hline 3 & mid-range & coil & 18.0 & 5.0 & 23.0 \\
\hline 4 & economical & coil & 18.0 & 0.0 & 18.0 \\
\hline 5 & foam & foam & 15.2 & 0.0 & 15.2 \\
\hline 6 & foam cushions & foam & 15.2 & 0.0 & 15.2 \\
\hline 7 & sofa bed & foam & 15.2 & 0.0 & 15.2 \\
\hline 8 & institutional & foam & 15.2 & 5.0 & 20.2 \\
\hline 9 & futon & futon & 15.2 & 0.0 & 15.2 \\
\hline \hline
\end{tabular}

respibands across the abdomen and chest during this experiment. The participant had a weight of $49 \mathrm{~kg}$ (107 lbs), and a height of $162 \mathrm{~cm}$ (5'4"). She followed a script of five actions: thirty seconds of deep breathing, thirty seconds of shallow breathing, a 10-20 second simulated apnea, an arm movement, and three leg twitches. Before and after every action, at least thirty seconds of quiet breathing was established. The script was performed once for each of three postures: supine, prone, and lateral (left or right). The full script, including all postures, lasted around 20 minutes.

\subsubsection{Multi-Participant Experiment}

This experiment was aimed at acquiring data for validation of algorithms in healthy populations. Fig. 3.4 depicts this experiment. A single institutional mattress on a low platform was used for this experiment. This mattress did not appear in the multi-mattress experiment. Two sensor mats were placed side-by-side, longitudinally below the mattress, centered below the pillow area for a total of 48 under-mattress 


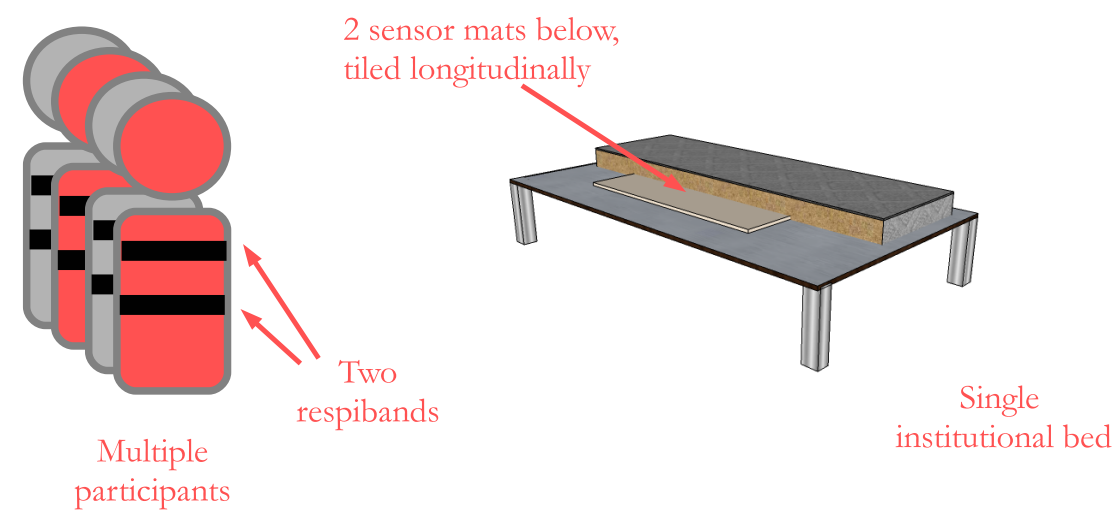

Figure 3.4: Components of the multi-participant experiment

Table 3.2: Multi-participant experiment: Participant information

\begin{tabular}{|r|r|c|c|c||c|}
\hline ID & Set & $\begin{array}{c}\text { Gender } \\
(\mathrm{M} / \mathrm{F})\end{array}$ & $\begin{array}{c}\text { Age } \\
(\text { years })\end{array}$ & $\begin{array}{c}\text { Weight } \\
(\mathrm{kg})\end{array}$ & $\begin{array}{c}\text { Height } \\
(\mathrm{cm})\end{array}$ \\
\hline \hline P02 & Train & Male & 33 & 76 & 170 \\
\hline P23 & Train & Female & 27 & 48 & 162 \\
\hline P44 & Train & Male & 32 & 90 & 172 \\
\hline P49 & Validate & Female & 27 & 64 & 170 \\
\hline P61 & Validate & Male & 27 & 95 & 180 \\
\hline P82 & Validate & Female & 31 & 62 & 162 \\
\hline P89 & Validate & Female & 24 & 63 & 162 \\
\hline P95 & Validate & Female & 30 & 66 & 170 \\
\hline \hline All & 3T/5V & $5 \mathrm{~F} / 3 \mathrm{M}$ & $28.9 \pm 3.1$ & $70.7 \pm 15.7$ & $168.9 \pm 6.2$ \\
\hline \hline
\end{tabular}

sensors.

Eight healthy participants wore respibands around their abdomen and thorax while performing a list of manoeuvres on the mattress with pressure sensors located below. Table 3.2 describes the participants, who were identified by a random two digit identification number and assigned to either a training (first three participants) or validation set (last five participants). 
Participants were requested to follow a script of breathing motions and muscular movements with two minutes of quiet breathing before and after each item. The script was: thirty seconds of deep breathing, thirty seconds of shallow breathing, move head, move head back, move arm, move arm back, move leg, move leg back, three leg twitches 10-15 seconds apart. and thirty seconds of simulated disordered breathing. The script was performed for each of four postures, with posture order selected by the participant: supine, prone, lateral left and lateral right. Once all four postures were completed, the participants were asked to lie quietly on the bed in postures of their choosing for the remainder of a two hour session. This last portion usually lasted around 20 minutes. During the experiment, a researcher annotated all movements and breathing patterns that were seen.

\subsubsection{Experimental Data Sets}

A number of data sets were extracted from the recordings made during the multipleparticipant experiment. Fig. 3.5 depicts the data sets that were created. To create data sets, all of the recordings from the experiment were segmented into 30 second epochs. The epochs overlapped by 27.5 seconds, with 2.5 seconds (25 samples) between the start of each epoch. This interval was chosen so that every epoch advanced by approximately half of a breath. 18123 epochs were established in the complete base set. 1470 epochs (8\%) were discarded since they either did not include any time in bed or did not have valid respiband data.

\section{Training, Movement, and Disordered Breathing Sets}

The first three participants were assigned to comprise the training set data for a total of 6937 epochs. The intent of this set was parameter optimization and/or supervised learning. Furthermore, from training set, breathing and movement-infected breathing sets were created. 


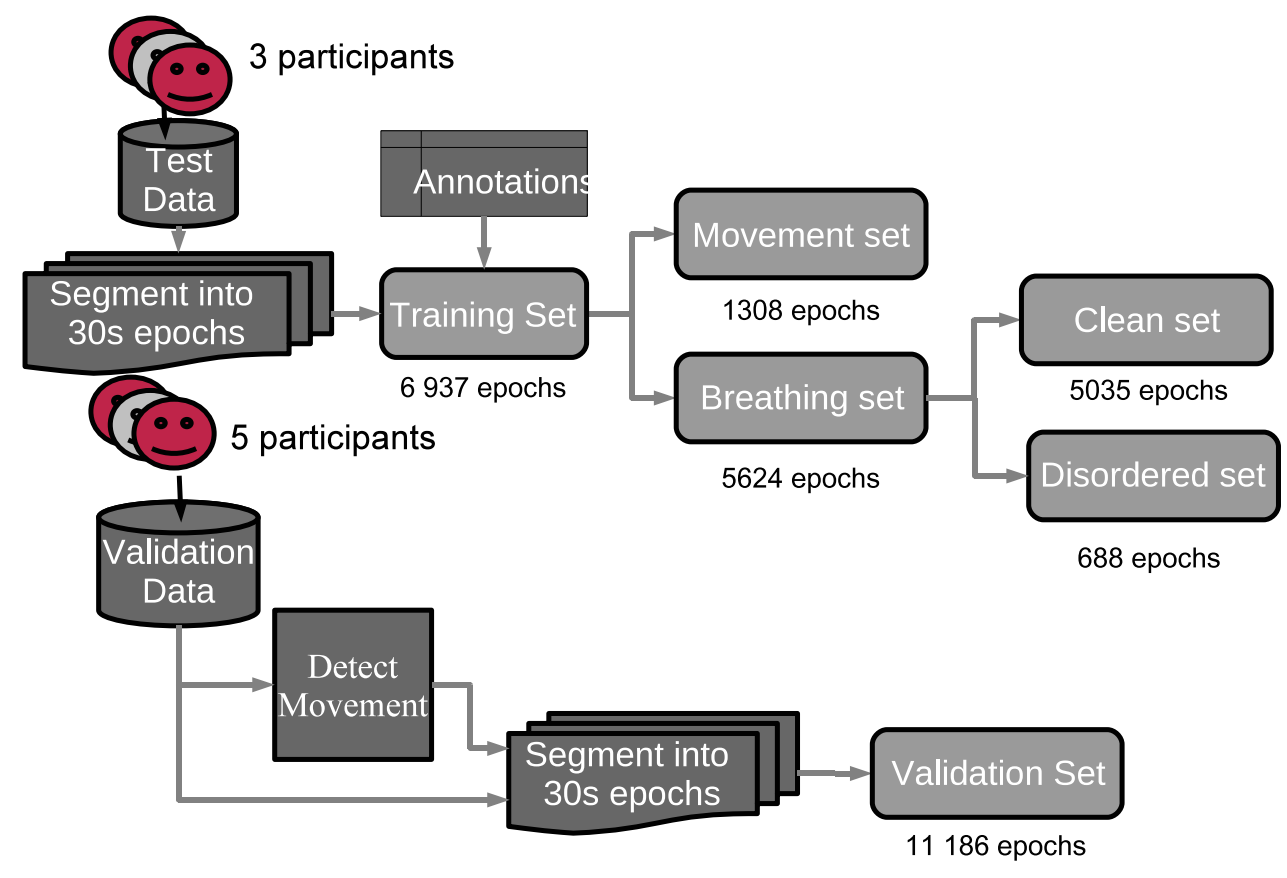

Figure 3.5: Data sets extracted from participant data

Movement annotations were used to split the training set data into 1308 epochs containing movement (18.9\%), 5624 epochs with valid respiband data and no movement $(72.0 \%)$, and a handful of discarded epochs due to unknown participant state.

The valid epochs with no movement comprised the breathing set. This set was further subdivided into clean and disordered sets. The clean set held epochs that contained only one type of breathing (regular, deep, or shallow) with 5035 epochs. Epochs in which breathing changed between regular and deep or shallow, and those in which disordered breathing was attempted by the participant, were assigned to the disordered set with 688 epochs.

\section{Corrupted Sets}

The clean respiration set with 5035 epochs introduced in the previous section, Section 3.3.4 was used to create simulated corrupted sets, to test basic ambient challenges of interference and reduced sensor availability. Fig. 3.6 depicts the three sets that 
were created by artificial corruption of these clean epochs. Details of each of these

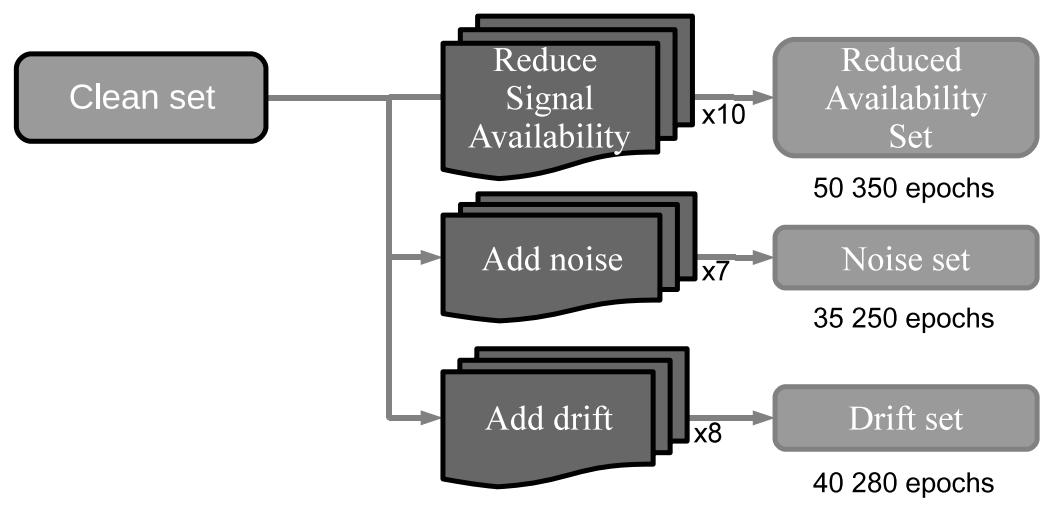

Figure 3.6: Data sets created by artificial corruption of clean respiratory epochs

corruptions follow.

To model reduced availability of breathing signals, $N_{k}$ of $K$ sensors were randomly chosen to be kept, while replacing the other $K-N_{k}$ sensors with a dummy AWGN noise signal. Note that the total number of sensors is kept constant, but the number of active sensors is reduced. $N_{k}$ was varied in 10 steps $\left(N_{k}=\right.$ $[1,2,3,5,7,12,15,20,30,48])$ and a total of 50350 epochs (5035 clean epochs $\mathrm{x} 10$ $N_{k}$ ) were created in the set.

To test the effect of interference, two corrupted data sets were created, noise and drift. The first set modeled reduced signal power with respect to noise power by adding additive white Gaussian noise (AWGN) to the epoch in proportion to each sensor's original standard deviation to provide a desired signal to noise ratio (SNR). Seven SNR were targeted (SNR $=[-22,-20,-17,-14,-10,0,10] \mathrm{dB})$, so that a total of 35245 epochs (5035 clean epochs x 7 SNR levels) were created in the set. The second set introduced drift into the epochs by adding AWGN that was lowpass filtered with cutoff frequency $0.07 \mathrm{~Hz}$, scaled in ratio to the variance of each sensor signal, forming a signal to drift ratio (SDR). The lowpass filter was a 256th order finite impulse response (FIR) filter with a cutoff frequency of 0.08 $\mathrm{Hz}$, designed using the window method with a Hamming window. Eight SDR ratios 
$(\mathrm{SDR}=[0.010 .10 .51241050] \mathrm{dB})$ were created for a total of 40280 epochs (5035 clean epochs $\mathrm{x} 8 \mathrm{SDR}$ ) in the set.

\section{Validation Set}

To validate algorithms that may have been tuned using the previously described test sets, a validation set was created from the data of the remaining five participants of the multi-participant study. As would occur in real-world usage, movement was not recorded from the manual annotations, but was instead automatically detected using a movement detection algorithm based on control limits, detecting movement when data points in a sliding window go outside of the mean \pm three standard deviations [124]. The raw data with the movement detections were segmented into epochs as described previously in Sec. 3.3.4. 11186 epochs comprised this set.

\subsection{Evaluation of Fused Respiratory Signals}

Respiratory signals are compared with respiband outputs in many sections of this thesis, notably in chapters 5, 6, and 7. In this work, the sum of the two RIP bands is used as the reference for relative breathing effort measurement. The sum of two RIP bands placed around the chest and abdomen was most similar to a gold standard pneumotachometer when compared to inductance plethysmography (IP) or a single respiband signal [140], Tidal volume can be accurately estimated using RIP sensors when properly calibrated in a controlled setting [141], [142], but accuracy decreases after positions changes [143], [144], so they are best used as semi-quantitative measures for tidal volume measurements [143]. Although RIP bands are alternative (but not recommended) sensors for airflow, calibrated or uncalibrated dual thorocoabdominal RIP belts are recommended for respiratory effort monitoring in sleep medicine [145]. Furthermore, they are preferred over piezoelectric or polyvinylidene fluoride (PVDF) 
belts for their documented ability to detect subtle respiratory effort [145].

\subsubsection{Comparison to Respiband Signals}

Output respiration signals and the respiband sum were post-processed similarly. First, segments between muscular movements were detrended and segments with movement were set to 0 . Movement occurrence was assigned according to the annotation files for the test data, and according to detected movements for the validation set. Then, the signals were filtered with a 128th FIR lowpass filter with cutoff frequency 0.8 $\mathrm{Hz}$, designed using the window method with a Hamming window. Edge effects from filtering were reduced by mirroring the first and last 128 samples of the 300 sample epoch before filtering and cropping to length once filtered.

To assess the quality of the output breathing signals, comparisons were made between the algorithmic output and the respiband standard using Pearson's correlation coefficient [146], denoted $r$. This coefficient provides a number in the range $[-1,1]$ and represents the strength of a linear relationship between the outputs of the proposed combiner and the respiband sum. A correlation of 1 indicates perfect increasing linear relation, -1 a decreasing linear relation, and numbers close to 0 indicate no linear relation. The strength of the relationship, but not the direction, is important in this case, so the absolute value of the correlation was used, $|r|$. This is akin to aligning reversals prior to comparison. Furthermore, a small phase difference can exist between the breathing signals from the respibands and the pressure sensors, so the two signals were first realigned, to a maximum of 2.5 seconds, using cross-correlation.

Along with the correlation coefficient, the percentages of $r>0.7$ are reported to provide an interpretation of the percentage of acceptable signals. While the 0.7 value is not an intrinsic cutoff, it provides signals with a coefficient of determination of $r^{2}>0.49$, so that generally half of the variation can be explained by the breathing signal. 
Pearson's correlation coefficient is a biased estimator of correlation, tending to slightly underestimate actual correlation due to a variance dependent on $r$ [147]. To reduce the effect of variance dependent on $r$, the Fisher transformation is often applied [147-149]. The idea is to transform the data to a domain where the variance is not dependent on $r$, calculate the desired statistic in the transformed domain, and then remap the interval to the regular domain using the inverse transform. While this method is useful for constructing confidence intervals with appropriately smaller upper intervals than lower intervals [147], it tends to overestimate bias correction when combining multiple correlation coefficients. Simple averaging without transformation is more accurate [150].

In this work, mean correlations, $|\bar{r}|$, were calculated following simple averaging. Plots involving mean values show confidence intervals using error bars. Confidence intervals of $|\bar{r}|$ at the $95 \%$ level $\left(\mathrm{CI}(|\bar{r}|)_{95}\right)$ were constructed using the one sample $t$ confidence interval [149].

$$
\begin{aligned}
C I(|\bar{r}|)_{100(1-\alpha)} & =\left(c_{\text {low }}, c_{\text {high }}\right) \\
& =\left(|\bar{r}|-t_{\alpha / 2, N_{e}-1} \frac{s}{\sqrt{N_{e}}},|\bar{r}|+t_{\alpha / 2, N_{e}-1} \frac{s}{\sqrt{N_{e}}}\right)
\end{aligned}
$$

For these intervals, $s$ is the sample standard deviation, calculated from the square root of the variance of the $N_{e}$ samples of $|r| . t_{\alpha / 2, N_{e}-1}$ denotes the $t$ distribution, with $\alpha / 2=0.025$ for the $95 \%$ level. The $t$ confidence interval requires a normal distribution for $|\bar{r}|$, which is provided by appealing to the Central Limit Theorem [149].

Since the Central Limit Theorem is only applicable when the available number of data points is sufficiently large, a second method of calculating $\mathrm{CI}(|\bar{r}|)_{95}$ was used when the available number of epochs $N_{e}$ was low. For $N_{e}<30$, bootstrap confidence intervals could be obtained using the MATLAB function bootci with 100 bootstrap 
samples. However, there were few calls to this method, as analyses that were performed in this work usually included more than 30 samples.

Significance of the results are tested using both paired and unpaired Student's $t$ tests with a confidence level of $95 \%$. When looking for improvements in the results for new algorithms over other algorithms, right tailed tests are performed. In some instances, multiple $t$ tests are performed to compare a method to multiple other methods. In such instances, the multiple comparison problem arises and the probability of committing at least on type I error by rejecting the null hypothesis when there is actually no difference in the mean rises. While there are methods to reduce this

chance, usually by increased stringency in the confidence level necessary to reject the null hypothesis, it is suggested that these methods are not consistent and that the original $t$ test is still the most powerful test [151]. In this thesis, no adjustment to the confidence level will be made for multiple comparisons, but $p$-values greater than 0.001 will be specified to provide information on the strength of evidence for rejecting a null hypothesis.

\subsubsection{Comparison to Respiband Respiratory Rates}

A secondary test to breathing quality analysis may also be applied by evaluating the impact of the extracted breathing signal quality on respiratory rate estimation. Respiratory rate was estimated from the average breathing interval as the reciprocal of the average breathing interval and multiplied by 60 to provide breaths per minute (BPM). To estimate the average breathing interval, individual breaths were detected and then the lengths of the intervals between detected breaths were measured in seconds. Intervals during which movement artifact occurred were discarded and the remaining intervals were averaged.

Breath detection followed a method proposed by Wilks et al. [152]. This method uses the beginning of upward and downward trends in the data to detect the breaths, 
eliminating those that do not alternate between upward and downward. A one second refractory period was also applied.

Power spectral methods for respiratory rate estimation, using the location of the fundamental frequency in the power spectrum, were also considered. However, benchtop testing revealed that the detection and interval method described above provided better agreement, particularly when there was movement in the epoch.

Rate estimation was applied to both the respiband signals and the output fused signals. Rate error was computed as the absolute difference between the two rate measurements. Also documented were: the availability of the respiratory rate (i.e. at least two breaths were detectable in the signal) expressed on a percentage basis, the mean absolute rate difference in BPM, the median and mean absolute errors expressed as a percentage of the breathing rate, and the percentage of rate estimations with absolute difference less than 1 BPM. Bland-Altman plots [153] to compare the two respiratory rate estimates were also useful in analysis. 


\section{Chapter 4}

\section{Characterization of System Response}

This chapter explores characterizing system response towards assessing feasibility

of signal extraction and linearizing its response. Ambient systems in homes will encounter a variety of furnishings and layouts. The unknown signal dampening that can exist between the sensor and the individual to be monitored may attenuate signals appreciably. This is explored in Sec. 4.2.

The material presented in Sec. 4.2 appeared in [49]. The work presented for array linearization (Sec. 4.3) was part of a larger work examining estimating contact force and location along grab bars. The work was included in three publications $[47,48,50]$.

\subsection{Introduction}

Previous research studies using pressure sensors to monitor physiological signs, such as breathing and heart rate, have often been in controlled laboratory or institutional settings. The effects of diverse living environments, where unobtrusiveness is achieved by embedding pressure sensors inside or below furniture, has yet to be explored. The following section quantitatively examines signals from sensors placed below a variety of mattress types and compares the response to signals from sensors placed on top of the mattress. Both available respiratory power and cardiac power are assessed. 
Not only does the environment in which sensors are placed affect response, but the sensor array itself plays a role. In Sec. 4.3, non-linear sensor response as well as nonuniform spatial response is explored. To this end, a theoretical model is proposed and applied to the specific sensors used for experimentation in this thesis. A calibration method, using this theoretical model, is then proposed for sensor arrays featuring non-linear and non-uniform spatial response.

\subsection{Experiment \#1: Environmental Response of Unknown Signal Dampening}

Data from the multi-mattress experiment, detailed in Sec. 3.3.2, was used in this experiment. The data was segmented by type of motion present into muscular movement segments respiration segments, and ballistocardiogram (BCG) segments. These last occurred during simulated apneas, when the cardiac signal was not overwhelmed by the respiration signal.

Signal powers of respiration and BCG segments were calculated according to the unbiased sample variance of each sensor signal during the segment:

$$
P=\frac{1}{N-1} \sum_{i=0}^{N-1}|x(i)-\bar{x}|^{2},
$$

where $x(i)$ is the sensor signal at the $i$ th sample of the $N$ samples in the segment, and $\bar{x}$ is the mean value over all $x(i)$.

The signal power loss due to attenuation was calculated. For each segment, a reference signal containing the strongest signal amongst all the sensor signals from above the mattress was chosen. Similarly, a reference signal was chosen from below the mattress. The average fraction of power available from the bottom reference signal with respect to the top reference signal was calculated as the mean power loss, 


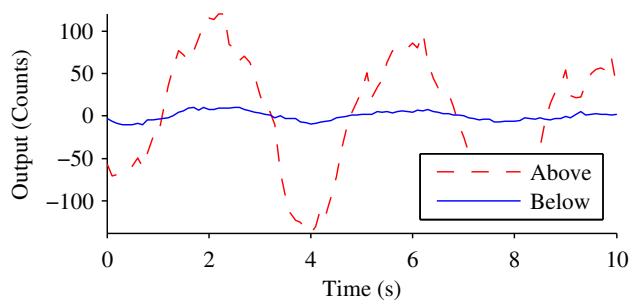

(a) Respiratory signal

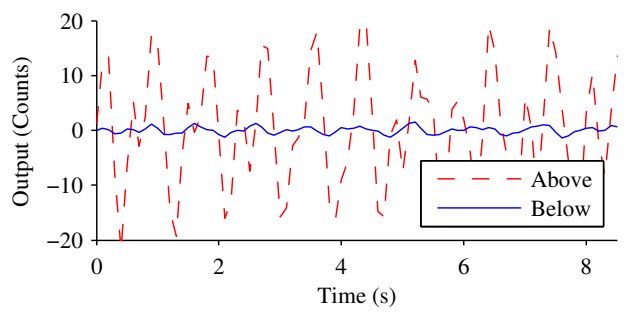

(b) BCG

Figure 4.1: Ten seconds of typical respiratory and cardiac signals recorded from above and below the deluxe mattress

$L$ across all $S$ segments. $L$ is measured in decibels:

$$
L=10 \log \left(\frac{1}{S} \sum_{s=0}^{S-1} \frac{\max \left(P_{\text {above }}\right)}{\max \left(P_{\text {below }}\right)}\right) .
$$

To compare power loss discounting the mattress thickness $t_{m}$, the power loss per centimetre of thickness was calculated as:

$$
\bar{L}_{\mathrm{cm}}=\frac{L}{t_{m}}
$$

The number of sensors from which the physiological signals were detectable also sheds light on the availability of these signals. The signals were defined as available when the signal power was greater than the noise power. Noise power was determined for each sensor from the variance of the sensor signals prior to loading.

\subsubsection{Results}

While the respiratory and cardiac signals were available under the mattress, their signal powers were attenuated. Typical respiratory signals and BCG signals sensed above and below a mattress are compared in Fig. 4.1. Fig. 4.2 plots the power loss $L$ as a function of the thickness of the mattresses and the power loss per centimetre of thickness of the component materials. As mattress size increases, generally more 


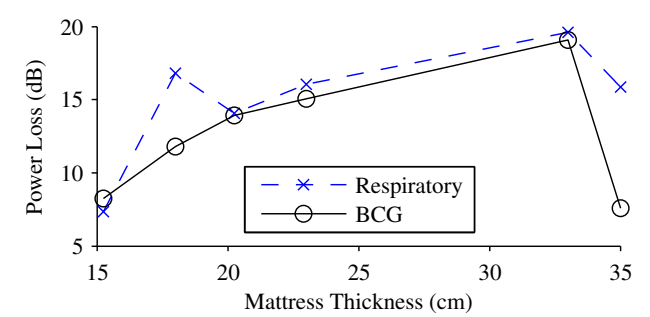

(a) Mean power loss with respect to mattress thickness

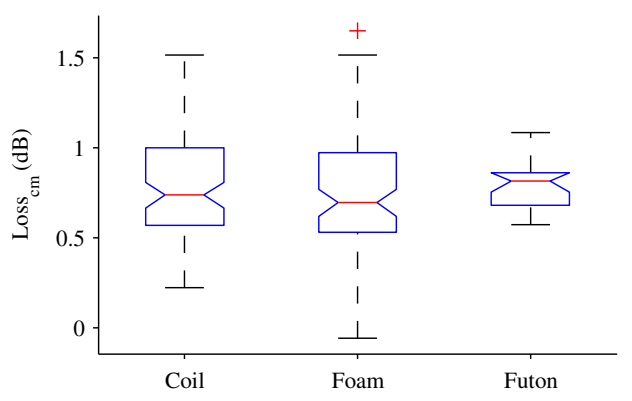

(b) Normalized loss by mattress material

Figure 4.2: Signal power loss with respect to thickness and mattress material

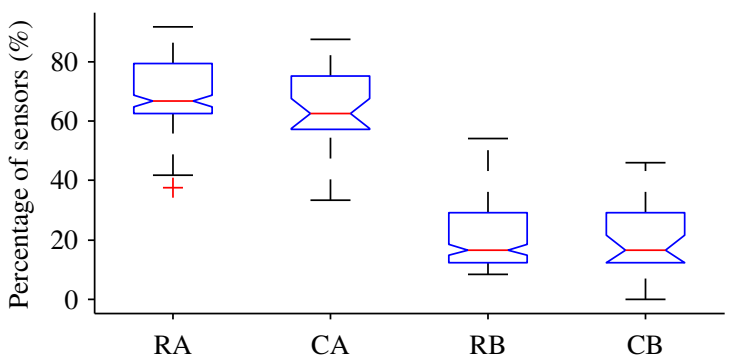

Figure 4.3: Boxplot representing the percentage of sensors with physiological signals above and below the mattress $(\mathrm{RA}=$ respiration above, $\mathrm{CA}=$ cardiac above, $\mathrm{RB}=$ respiration below, $\mathrm{CB}=$ cardiac below)

power is lost, although there are outliers, which may be due to the mattress construction and its component material(s). After normalizing for thickness, the futon showed the highest power loss per centimetre of thickness, with foam and coil both near a median of $0.7 \mathrm{~dB} / \mathrm{cm}$, but futon at $0.8 \mathrm{~dB} / \mathrm{cm}$.

The mattress distributed the load to more sensors. However, the signal loss due to the mattress overrode the load distribution effect. Fig. 4.3 shows a dramatic drop in the number of sensors reporting physiological signals below the mattress. On average, $69 \% \pm 12 \%$ of the sensors detected the respiratory signal above the mattress, and $22 \%$ $\pm 10 \%$ below. In all instances, at least two sensors detected the respiratory signal. Chen et al. [33] reported a wide range of positive predictivity on detected breaths $76 \%$ - 99\% when a differential pressure sensor was positioned in varying locations 
under a mattress of unreported type. The results here show much lower breathing signal availability, but the sensors here detect pressure over a smaller spatial area.

The BCG was available from at least one sensor for all mattresses and postures from above the mattress, and in $97 \%$ of cases from below. This compares closely with the over $97 \%$ positive predictivity reported by Chen et al. [33].

\subsubsection{Discussion}

Sensors placed below a mattress rather than above are less obtrusive, while retaining the potential for physiological signal monitoring. However, the signals can be heavily muted and may feature less distinct localized behaviour. More sophisticated signal processing algorithms may be necessary to cope with the lower signal to noise ratios than would be required from sensors located above the mattress.

The futon, which had the lowest signal transmission, represents the most dense of the mattress types. Watanabe et al. [23] also reported the lowest signal to noise ratios from a futon compared to three other mattress types. It is likely that the available power was not only affected by the thickness, but also the density of the material of the mattress. A model of signal transmission that includes both material and thickness could be useful to determining if a given mattress would be unable to reliably transmit the physiological signals.

Cardiac signal availability was assessed by the existence of a peak in the relevant spectral band. With this metric, it is possible that the system overestimates the availability of the cardiac signal, since small unconscious movements made during apneas would lead to over-estimating the cardiac signal power.

When multiple sensors are used, not only does each sensor detect differing breathing amplitudes, but also the number of sensors exposed to the breathing signal varies. An ambient system must cope with low levels of captured breathing signal power compared to noise power and a varying numbers of sensors with detectable signals. 

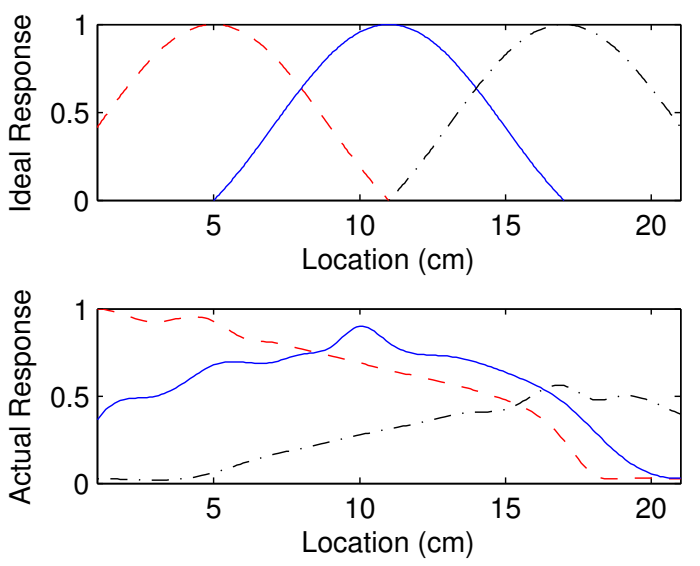

Figure 4.4: Ideal response of sensors in an array (top) and response of actual sensors in non-uniform pressure sensor array (bottom)

\subsection{Characterization of Non-Uniform Sensor Ar- ray Response}

In an array configuration, spatial response differences between sensors play a role in system response. Ideally, normalized sensor response in an array is spatially uniform from sensor to sensor, with distinct overlap and uniform gain [154]. However, membrane deformation, sensor manufacturing variability, wear and tear, or other conditions may contribute to a non-uniform spatial response. Fig. 4.4 depicts the spatial impulse response from an idealized array in the top plot, while the bottom plot depicts the response from an array with non-uniform spatial response, differing gain responses to the same applied force, increased sensitivity some locations, and edge effects. In this type of array, small displacements of weight can affect force estimates. Indeed, such spatial dependencies have been observed before from pressure sensor arrays [155], [156].

A theoretical non-linear model of the sensor is created to characterize the sensor response in the following section. Using this theoretical model, non-uniform spatial response is addressed in Sec. 4.3.2. 


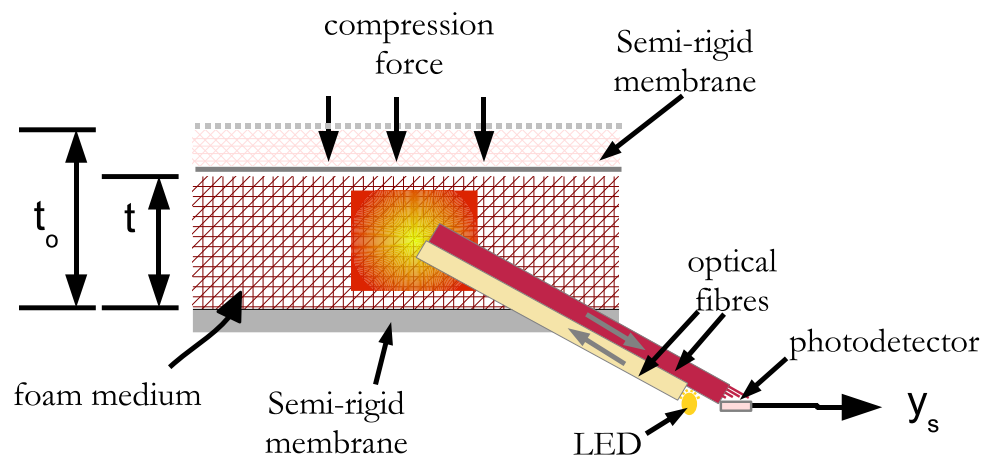

Figure 4.5: The Kinotex ${ }^{\mathrm{TM}}$ Sensor

\subsubsection{A Theoretical Model for Kinotex ${ }^{\mathrm{TM}}$ Sensors}

The Kinotex ${ }^{\mathrm{TM}}$ sensors introduced in Sec. 3.1.1 have a very non-linear response. This response can be modeled theoretically by examining the materials and physics of the sensor.

Fig. 4.5 presents a cutaway view of a Kinotex sensor. In this sensor, a transmitting optical fibre introduces light into a foam medium. Some of this light is transmitted through the foam, while the rest is scattered and reflected. A second receiving fibre, placed directly next to the transmitting fibre, receives the reflected light and returns it to a photodiode for conversion into voltage. As the foam is compressed, its density increases, decreasing the transmittance and increasing the amount of reflected light.

The same principle that is used by the Kinotex ${ }^{\mathrm{TM}}$ sensor has been used in industrial processes to accurately measure foam thickness [157]. When light shines through a uniform foam medium, the intensity of light that is transmitted through the medium can be expressed by Lambert's Law:

$$
I=I_{0}\left(e^{-\alpha t_{f}}\right)
$$

where $\alpha$ is the absorption coefficient, $t_{f}$ is the foam thickness, and $I_{0}$ is the intensity of the light applied to the foam. 
For the Kinotex ${ }^{\mathrm{TM}}$ sensor, reflected rather than transmitted light is measured, but a similar model can be applied. If all of the light that is not transmitted is reflected, the response, under pressure, is the full original light intensity minus the transmitted light:

$$
I=I_{0}\left(1-e^{-\alpha \overline{t_{f}}}\right) .
$$

Here, thickness is replaced by the normalized foam thickness, referenced from the initial thickness, $t_{0}$, and new thickness $t_{f}: \overline{t_{f}}=t_{f} / t_{0}$. Pressure affects normalized foam thickness according to [158]:

$$
\frac{t_{f}}{t_{0}}=(1-r) e^{-a_{c} p}+r,
$$

where $r$ is the foam's compressibility factor, $a_{c}$ is the foam's compliance factor, and $p$ is the applied pressure.

By substituting the normalized thickness from (4.6) into (4.5) and collecting constants into new constant factors, the equation relating input pressure to output voltage can be reduced to:

$$
\ln \left[x_{0}-x\right]=c_{1} e^{-c_{3} p}+c_{2},
$$

where $c_{1}, c_{2}$, and $c_{3}$ are unknown constants, voltage outputs $x$ are proportional to light intensities $I$, and $x_{0}$ is the zero-value voltage. A value for $y_{0}=2.8 \mathrm{~V}$ was set from the maximum output voltage that occurred across numerous sensors. For a contact area $A$ that is small and constant, $f=p A$, and force can be substituted for pressure in the model, with the constant $c_{3}$ absorbing $A$.

To linearize the sensor response, the inverse model of 4.7 can be applied to the inputs from the sensors. This inverse model is a strictly increasing continuous nonlinear function:

$$
\hat{f}^{\prime}=p^{-1}(x)=-\frac{1}{c_{3}} \ln \left(\frac{\ln \left[x_{0}-x\right]-c_{2}}{c_{1}}\right) .
$$




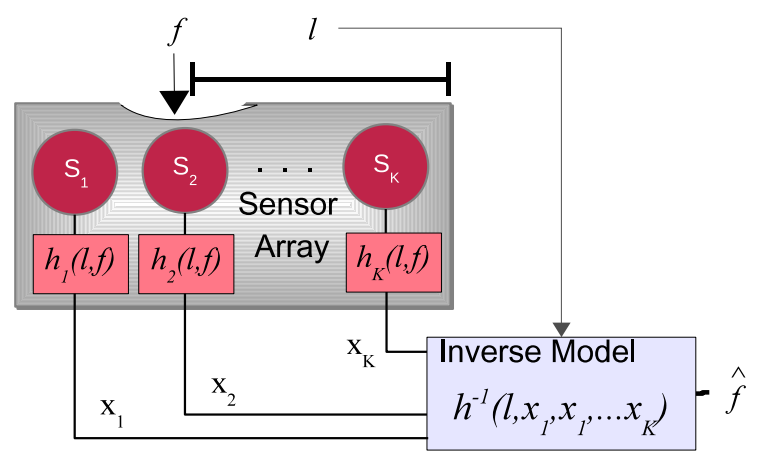

(a) Linearization architecture

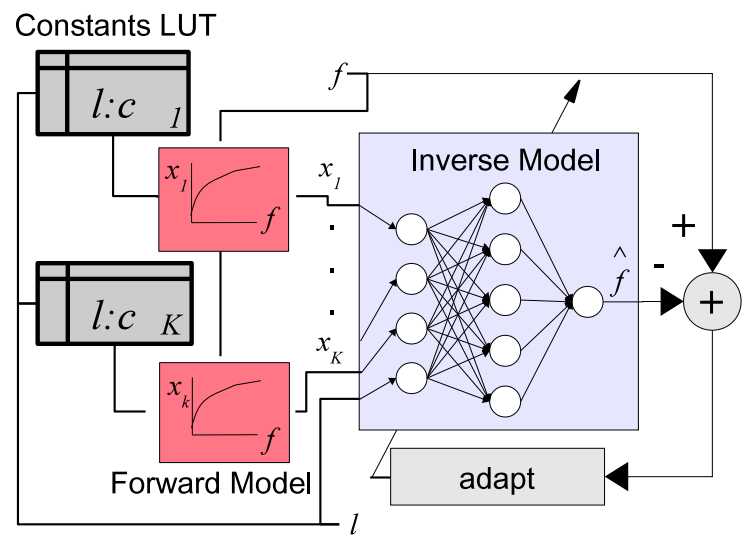

(b) Training the ANN with modeled data

Figure 4.6: Block diagrams of linearization through modeled calibration data

To fit the forward model, a set of constants was determined for each sensor individually at the given location of applied force. This provided a non-linear model curve for each sensor and location and the constants for this curve were saved in a lookup table. Using the curves, artifical calibration data can be created by pairing points along the curve. To find the constants, calibration data was fit to (4.7) using a nonlinear curve fitting routine. MATLAB's fminsearch function performs multidimensional nonlinear minimization, and was used to find $c_{3}$. Once the best fit for this constant was found, the other constants were found through least squares optimization.

\subsubsection{Method: Calibrating with Non-Uniform Spatial Re- sponse}

The problem of estimating applied force from the pressure sensor signals is examined. Fig. 4.6 presents a block diagram of force estimation from an array with both nonuniform spatial response and non-linear sensor response.

The pressure sensor array consists of multiple pressure sensors positioned far enough apart so that loads are expected to be exerted between sensor elements or covering just one element, while individual sensors may respond to forces applied a 
number of sensors away. The sensors convert the applied force to a voltage. Modeling conversion of known forces into voltages is considered the forward model. To estimate the force applied to the sensor array, it was necessary to create an inverse model of the array response. By using this model, acquired voltages were converted back to applied force. Artificial neural networks (ANN) have been used to linearize pressure sensors affected by both the intrinsic non-linear response and a second source of non-linearity [159]. While the second source of non-linearity was temperature in that method, here it was applied to the spatial response by providing the location as an input to the ANN. Furthermore, the ANN can be used to fuse the results from an array of sensors to estimate the applied force by also using all of the array inputs. Normalized sensor values were input to an ANN, along with the location of application, and the ANN computed the estimated applied force.

An ANN must be trained first with known input and output tuples. These tuples need to cover the full range of expected force and location estimates. This can be done by taking a large number of calibration points, over many force and location pairs. However, as this type of calibration is time consuming, fewer calibration points were obtained for the training set. Synthetic data was created between these points through interpolation of the theoretical model to augment the set. Constants were fit to the calibration data to form the model and then modeled data was input to the ANN for training. Fig. 4.6b shows this training.

Interpolating the training data can be done between the weight values or across the locations. Splining across the location values was first trialed, but tended to make the ANN worse at predicting force than if only the few calibration points were used to train the ANN. Instead, the developed optical pressure sensor model was used to interpolate data points across the force values. A forward model was fitted to each sensor output at each of the locations given by the basic set and the model is sampled at regular intervals of force to determine the expected output of the sensor 


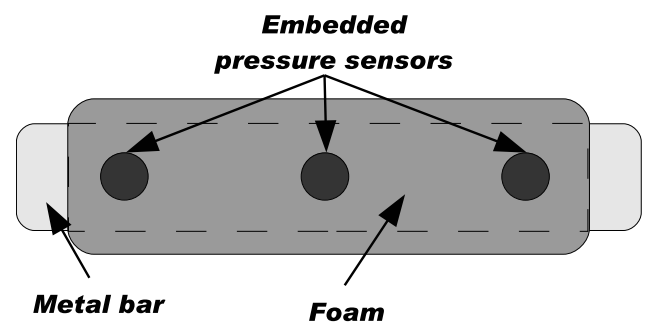

(a) Top View

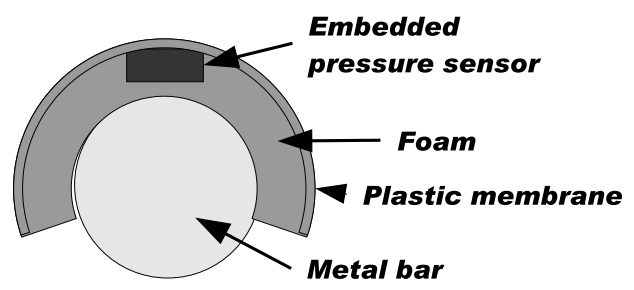

(b) Cutaway View

Figure 4.7: Top and cutaway views of the array

under these forces. It contained more unique forces than the basic set, but at the same locations.

\subsection{Experiment \#2: Linearization of Non- Uniform Sensor Arrays}

To assess the calibration methods, a non-uniform one-dimensional array of optical pressure sensors was formed and installed across a metal bar. Calibration and test set data were collected from the array and developed methods were used to linearize the response.

Three Kinotex ${ }^{T M}$ sensors, $0.9 \mathrm{~cm}$ in diameter, were embedded in polyurethane foam and affixed to the top side of a metal bar. The length of the bar was $21 \mathrm{~cm}$ and the sensors were placed at $5 \mathrm{~cm}, 11 \mathrm{~cm}$ and $17 \mathrm{~cm}$. The array and bar were then covered in a sheet of semi-rigid plastic, such that the force applied at any location along the bar would activate at least one neighbouring sensor in the array. Fig. 4.7 presents a diagram of the array on the bar.

Calibration data was acquired by using known weights hung from a $1 \mathrm{~cm}$-wide ribbon, looped over the bar. The ribbon was moved along the bar in $1 \mathrm{~cm}$ intervals from locations $1 \mathrm{~cm}$ to $21 \mathrm{~cm}$ along the bar. This was repeated three times with different weights, whose masses were $1 \mathrm{~kg}$ (weight $9.8 \mathrm{~N}), 2.5 \mathrm{~kg}(24.5 \mathrm{~N})$, and $5 \mathrm{~kg}$ 
(49 N). This created 63 input location and force pairs. At each weight and location, 50 output samples were recorded at $10 \mathrm{~Hz}$ (1 second of data). A further 150 samples were recorded without any applied weight to obtain data with a force of $0 \mathrm{~N}$.

Test data was then acquired by recording 50 samples at each of 30 randomly chosen location and weight pairs. Location values of $4.9 \mathrm{~cm}, 10.2 \mathrm{~cm}, 13.5 \mathrm{~cm}, 18.7 \mathrm{~cm}$, and $20.0 \mathrm{~cm}$, each with applied weights of $0 \mathrm{~N}, 4.9 \mathrm{~N}, 9.8 \mathrm{~N}, 14.7 \mathrm{~N}, 29.4 \mathrm{~N}$, and $39.2 \mathrm{~N}$ were sampled. The location values were chosen randomly, although it was ensured that all but one of the weight-location pairs would not be found in the calibration data.

To evaluate the theoretical sensor model without spatial response, the 200 weight and output calibration tuples at the location directly on top of each sensor were used to fit the model. This fit was then compared to a linear and second order polynomial fit. The training sets for the ANNs split the calibration samples, averaged over each location and force, into training and validation sets. The basic set had 84 tuples, split into 80 tuples for training and 4 tuples for validation. The model-based synthetic ANN training set contained 496 training tuples and 30 validation tuples. After optimizing, the ANNs were constructed with four input nodes, nine hidden nodes and one output node. The activation functions were a hyperbolic tangent in the hidden layer and a linear function in the output layer.

The ANNs were trained for a maximum of 50 epochs and their outputs were verified after every epoch against the validation set. Once validation set error started rising, indicating overfitting, the training was halted. The network with the median test error was selected for result reporting.

The proposed ANN method was compared to a spatially uniform model that ignores spatial non-uniformity. Using an idealized response function, it remaps the output according to the location of force and then uses the theoretical sensor model to estimate applied force. Output applied force was averaged from the two closest sensors. These were also compared to an ANN trained only on the calibration points 


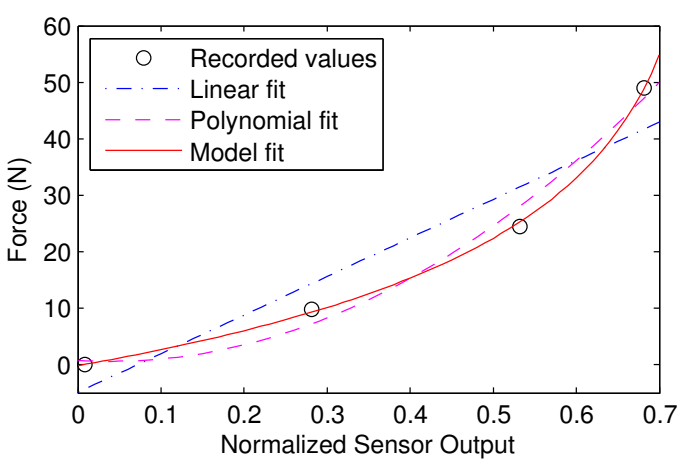

(a) Example models of inverse response

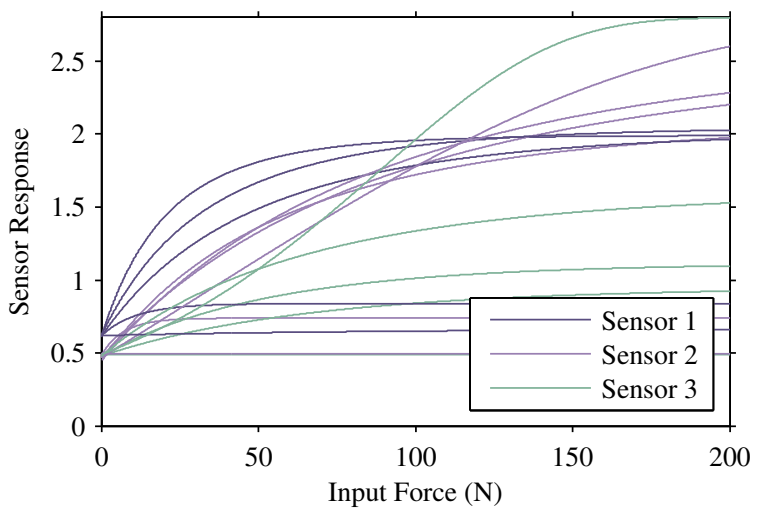

(b) Forward theoretical models of pressure sensor response observed along the bar

Figure 4.8: Forward and inverse models of sensor response. Left: theoretical responses along the bar. Right:Examples of inverse modeling

instead of the synthetic modeled data.

\subsubsection{Results: Calibration}

Fig. 4.8b shows some of the response curves for the forward model of the fitted theoretical constants across the bar and Fig. 4.8a shows the modeled pressure sensor response to forces applied on top of the sensor. The linear fit, second order fit, and theoretical model fit are shown. The three sensors were each modeled in this manner, and the theoretical models provided better fitting than the polynomial models. However, the response of each sensor varied considerably and they each required a unique fit.

Force estimation bias and root mean square error (RMSE) were measured, comparing three methods of inverse modeling. The spatially uniform model uses only the calibration values taken from directly on top of each sensor to model that sensor and then assumes an ideal response to model the effect of the location. The second and third use an ANN to model the response, with the second one using just the calibration data points for training and the third one using synthetic data from the fitted 
Table 4.1: Error statistics for given methods

\begin{tabular}{|r|c|c|}
\hline Method & Bias [N] & RMSE Error [N] \\
\hline \hline Spatially Uniform Model & 1.91 & 3.6 \\
\hline Basic ANN & 0.25 & 4.0 \\
\hline Model-Trained ANN & 1.32 & 2.5 \\
\hline
\end{tabular}

models at the calibration point locations. These are displayed in Table 4.1. Bias is a measure of the mean error and indicates if a method is biased towards reporting forces that are higher or lower than the actual force. A small amount of RMSE is expected across all methods due to systemic noise in the sensor outputs. The bias and RMSE error can also be viewed as the mean and standard deviation of the error. All of the methods were positively biased, indicating that the estimated force underreported the actual applied force, especially for the spatially uniform model method. However, the theoretical model had better accuracy than the polynomial method, but the model-trained ANN performed best, by taking spatial response into account. The basic ANN, using only calibration data for training, was poorest at estimating force.

\subsubsection{Results: Consequences for Breathing Fusion}

If linearization is not performed, fusion must be performed on the non-linear input signals. Fig. 4.9 shows how a breathing signal shape and amplitude can be influenced by the basic loading on the sensor, using the modeled response curve of sensor 2 at $20 \mathrm{~cm}$. The input breathing effort signal is shown with time on the y-axis in order to align the input force with the input of the plot above it, to show the deviation due to mean loading along the response curve. Of particular interest are the two input sensor signals loaded at 100 and $180 \mathrm{~N}$. While they were produced by the same input breathing signal and are aligned in time, their shape makes it seem as if one is 


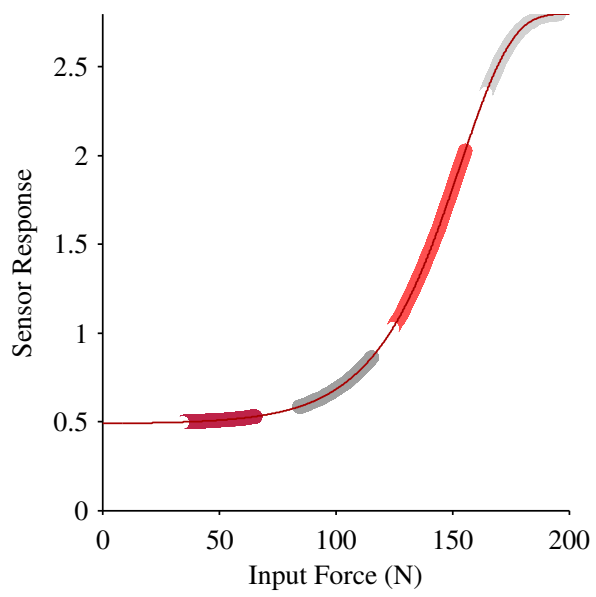

(a) Sensor response

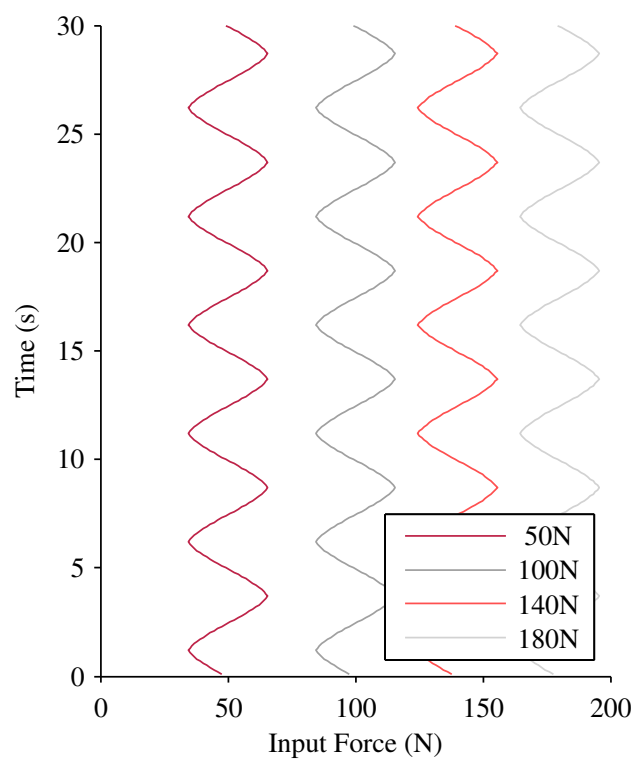

(c) Breathing effort

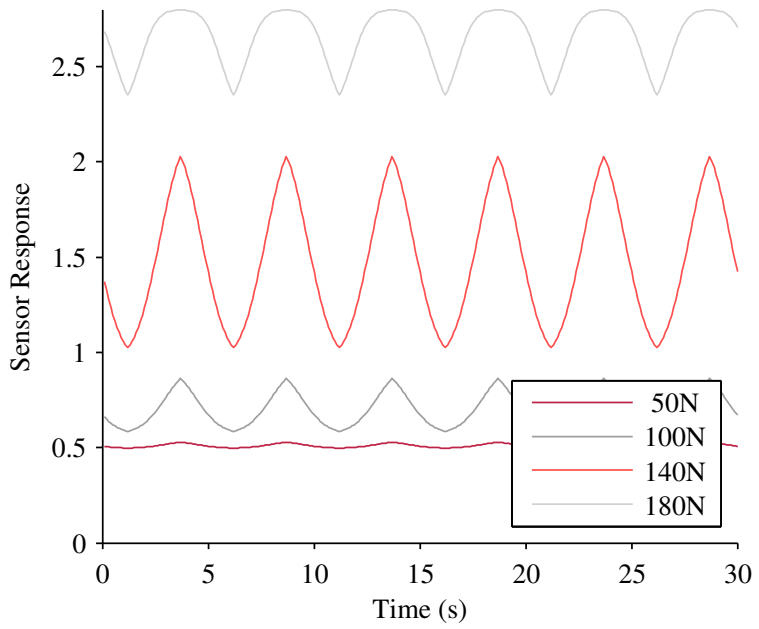

(b) Input sensor signals

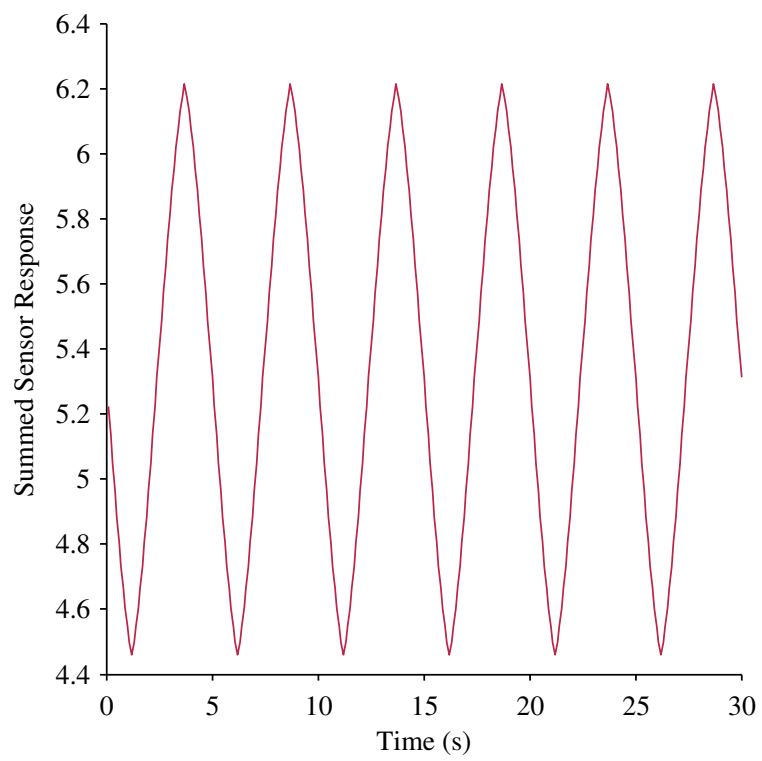

(d) Fused (summed) breathing signals

Figure 4.9: Effect of sensor response on the shape of a simulated breath signal according to four values of mean loading: [50, 100, 140, 180] N. 
polarity reversed (flipped) and out of phase by a quarter period. Polarity reversals are common during breathing on the sensor array in bed. The small weight shifts that produce breathing add load to one sensor while reducing load on another. However, for this case reversal should not be considered.

The fusion by simple summation of all of these sensor signals results in a signal that is less non-linear than the average input as the non-linearities tended to cancel each other. With a diversity of loading levels on the bed, a summed output signal may better represent the input breathing effort than individual sensors would.

\subsubsection{Discussion}

The response of Kinotex ${ }^{\mathrm{TM}}$ sensors to applied pressure or force contains two sources of non-linearity: the response of the light to foam thickness and the response of the foam to applied force. By fitting the total response to a theoretical model, a closer fit is achieved than with generic polynomial models.

Methods of obtaining a force estimate from a pressure sensor array that exhibits non-uniformity were investigated. While it can be expedient to ignore the spatial response and assume uniformity, more accurate results were obtained by modeling the spatial response using an ANN. However, it was important that the ANN be trained on enough data, either through longer calibrations or by synthetically creating data by fitting a theoretical model to the data and sampling the model.

Calibration enables accurate pressure or force measurement, but the manual work required to perform the calibration procedure is lengthy. Every calibration data point requires waiting for a settling time and an adequate number of samples for averaging before moving on to the next location. Although interpolating the data allows fewer calibration points, resolution and accuracy is ultimately determined by the number of points. For two dimensional arrays, the calibration procedure would be exponentially longer. If the technology is to be deployed on a large-scale basis, such manual work 
would not be cost effective. Rather than explore these methods, the rest of this thesis will assume uncalibrated, non-linear data. By developing processing algorithms that use the sensor outputs as semi-quantitative measures of breath amplitude, which do not require exact measurements of pressure, the difficulties and time consumption of calibration can be avoided.

The manufactured BOS sensor pad used for bed-based experimentation shows more spatial uniformity than the ad-hoc array configuration here. However, the non-linear effects are certainly present, and breathing signals with the sensor pad sometimes did show the types of signal shapes shown in Fig. 4.9. The next chapter explores breathing signal alignment. Since polarity reversal were observed to be more common than signal delays between sensors and there is a risk of improperly flipping and delaying signals due to the non-linear shapes of the curves, only reversals will be considered.

\subsection{Summary}

The experiments run in this chapter explored characterization of a sensor array and of signal loss in ambient environments. Some of the conditions that create variability in an ambient system were observed, including differentials in the response of sensors, unknown signal to noise power, and the availability of a given physiological signal at a given sensor. Both availability of breathing and breathing power diminished as a function of mattress size, although composition of the mattress and its density may play a role as well. When developing algorithms for physiological monitoring, it is important not only to test an algorithm in a laboratory environment, but also to make sure that it is robust to these real-life conditions. Sensor response can be affected by a number of non-linearities and non-uniformities, including the sensor material properties and the spatial response between sensors. While it is possible 
to calibrate a sensor array to linearize the response, calibration is a time consuming proceduce. It was discovered that uncalibrated outputs could produce aligned signals that seemed unaligned by virtue of the sensor response. During signal alignment, delay measurements between sensors may be suspect. 


\section{Chapter 5}

\section{Reversal Detection}

Chapter 2, Sec. 2.2.3 raised the requirement of alignment of multisensor signals by reversal detection and correction. This chapter examines improving reversal detection by directly addressing reversals. Furthermore, it explores the impacts of fusing rather than selecting the reference signal that is required in this process. The impact of preconditioning by lowpass filtering is also assessed.

This chapter proposes methods of reversal detection and correction that directly assess reversals, rather than conventional techniques that use more oblique methods of time delay estimation. The multisensor signal reversal model is first constructed in Sec. 5.2. The signal model is applied to reversal detection using correlation in Sec. 5.3.1. A correlation coefficient method was tested in [52] and was subsequently enhanced by Daphne Townsend to add time delay alignment to the reversal detection. The latter technique was published in [53]. Sec. 5.3.2 addresses the multisensor signal reversal model using short-term trend analysis with fused reference signals. Both segment-based and adaptive methods are proposed. A preliminary version of the adaptive short-term trend algorithm was published in [52].

Parameter selection in the presence of additive white Gaussian noise (AWGN), drift interference, and varying signal periods is explored by simulation with generic periodic signals in Sec. 5.4. Simulation is also used to compare the resistance of the 
optimized algorithms and conventional traditional cross-spectral phase (Spec. Phase) analysis to varying levels of interference and jitter in the signal period.

Sec. 5.5 revisits the parameters selection for application to breathing signal monitoring. Furthermore, the effect of pre-conditioning the signals and fusing the reference signal are also quantified. Results are validated with experimental data for comparison across algorithms.

\section{$5.1 \quad$ Introduction}

When multiple respiratory signals are combined using summation, they need to be coherent, both aligned in time and in polarity. Fig. 5.1 depicts two polarity-reversed outputs from a pressure sensor array, once detrending removed the loading component. The sensor locations are indicated by the larger and brighter red and gray

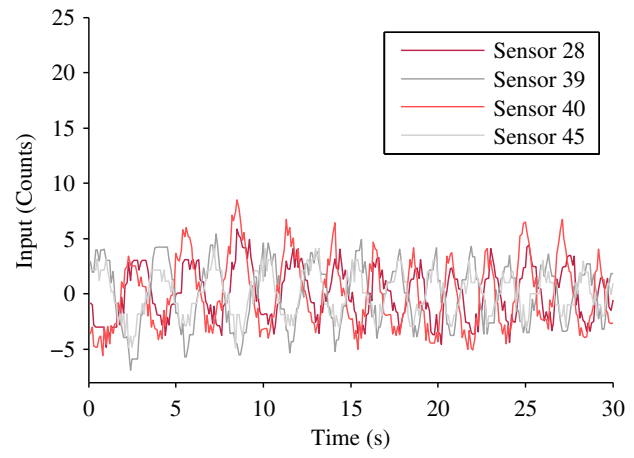

(a) Signals

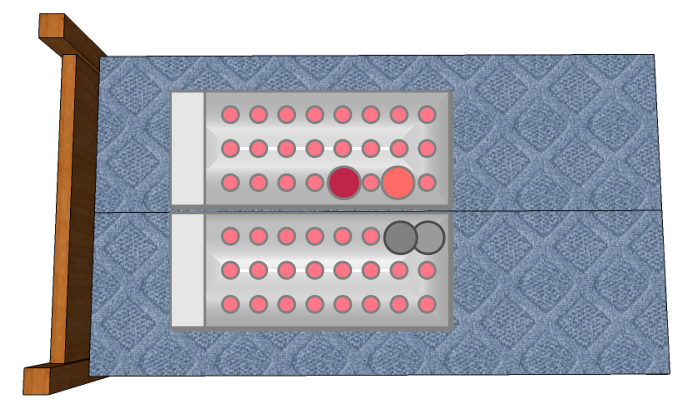

(b) Sensor Locations

Figure 5.1: Two detrended respiratory signals from a pressure sensor array with similar amplitude but reversed polarity

circles, corresponding to the colours of the plot. The red signals rise together while the gray ones fall together. Although the participant is prone, a side-to-side reversal seems in effect, possibly because of a slight twisting of the body towards one side to breath. 


\subsection{Multisensor Reversal Detection Signal Model}

Let $z(t)$ be the underlying signal of interest under measurement by $K$ sensors. Its sampled version is $\mathbf{z}=\left[z\left(t_{1}\right) z\left(t_{2}\right) \ldots z\left(t_{N}\right)\right]$. The input $\mathbf{x}_{\mathbf{k}}=\left[x_{k}(1) x_{k}(2) \ldots x_{k}\left(t_{N}\right)\right]$ from sensor $k$ is modeled by

$$
\mathbf{x}_{\mathbf{k}}=p_{k} g_{k}(\mathbf{z})+\epsilon_{\mathbf{k}}+v_{k} .
$$

The response of the sensor and other systemic responses that take place between the signal origination and the digital system input is encapsulated by $g_{k}(\cdot)$. This function must be strictly increasing in order to preserve stimulus response and is often non-linear for most sensor types. Reversals are modeled by polarity variables $p_{k} \in\{-1,0,1\}$, where 1 indicates no reversal, -1 indicates a reversal, and 0 indicates the signal is not available from that sensor. Interference is represented by $\epsilon_{\mathbf{k}}$, including sensor noise, drift, and burst interference. A baseline DC constant is provided by $v_{k}$.

The goal is to find a polarity $p_{k}^{\prime} \in\{-1,1\}$, where $p_{k}^{\prime}=C p_{k}$ for all $p_{k} \neq 0$ and $C$ is constant $\in\{-1,1\}$. Selection of $p_{k}^{\prime}$ is generally performed by generating a single reference $\mathbf{x}_{\mathbf{r}}$, comparing it to each $\mathbf{x}_{\mathbf{k}}$, and selecting $p_{k}^{\prime}$ according to a binary

comparison output. With $p_{k}$ appropriately selected, all $\mathbf{x}_{\mathbf{k}}^{\prime}=p_{k}^{\prime} \mathbf{x}_{\mathbf{k}}$ are coherent and an approximation $\mathbf{z}^{\prime}$ of the original $\mathbf{z}$ can be formed by summation:

$$
\mathbf{z}^{\prime}=\sum_{k} p_{k}^{\prime} \mathbf{x}_{\mathbf{k}} \underset{\sim}{\sim} \mathbf{z}
$$

If $C=-1, \mathbf{z}^{\prime}$ will be reversed from $\mathbf{z}$ and inspiration is indicated by a falling signal and expiration by a rising signal. If the $p_{k}$ are chosen poorly, the output signal may still approximate $z$, but would represent a poorer approximation as destructive interference occurs and the interference signals are relatively stronger. 


\subsection{Methods of Reversal Detection}

\subsubsection{Correlation Analysis for Reversal Detection}

A related field for reversal detection is in detection of paradoxical motion from respibands when asynchrony occurs between thoracic and abdominal effort. Paradoxical motion is an indicator of obstructive apnea, occurring in one study in $91 \%$ of patients during obstructive apneas [160]. Prisk et al. [161] examined six methods to detect asynchrony from the two traditional respiband signals in the time-domain and concluded that cross correlation and maximum linear correlation performed best. In reversal detection, only the sign of the correlation coefficient is required rather than complete cross correlation.

To detect reversals with the correlation coefficient, a reference sensor is first identified, as in Nishida et al.'s method for reversal detection [15]. Pearson's correlation coefficient is then calculated to compare the reference sensor input to all other sensors. Let $r_{k}$ be the coefficient between the reference sensor's input $x_{r}$ and the $k$ th sensor input $x_{k}[146]$ :

$$
r_{k}=\frac{\sum_{j=1}^{N_{s}}\left(x_{r}(j)-\overline{x_{r}}\right)\left(x_{k}(j)-\overline{x_{k}}\right)}{\sqrt{\sum_{j=1}^{N_{s}}\left(x_{r}(j)-\overline{x_{r}}\right)^{2}} \sqrt{\sum_{j=1}^{N_{s}}\left(x_{k}(j)-\overline{x_{k}}\right)^{2}}} .
$$

The numerator represents the covariance between the two signals and the denominator represents scaling each by their standard deviations. Positive correlations infer the sensor inputs are both rising or falling, while negative ones infer one is rising while the other is falling. Thus, $p_{k}$ can be declared as the sign of $r_{k}, p_{k}=\operatorname{sgn}\left(r_{k}\right)$.

Although Pearson's correlation coefficient measures linear relationships, the sign of non-linear relationships can be properly detected, as long as the relationship still 
is strictly increasing or decreasing. Fig. 5.2 depicts correlation as a linear regression between the reference and the sensor signals [146]. Sensor 28 is used here as the reference signal and all are scaled first by their standard deviations. The correlation of the

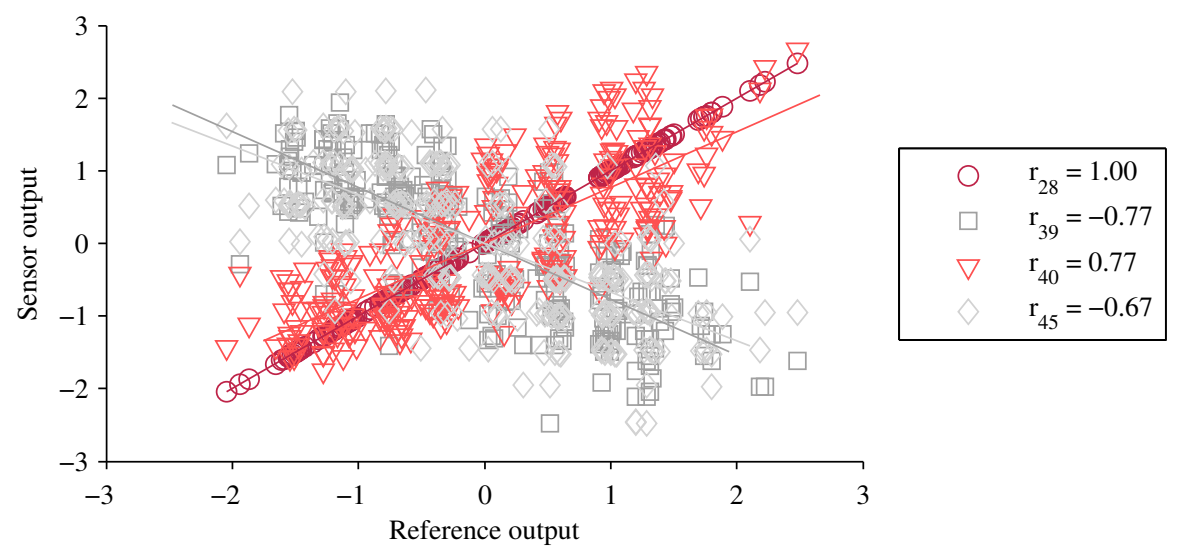

Figure 5.2: Pearson's correlation coefficient as a standardized linear regression

reference signal, sensor 28, with itself is a one-to-one mapping and results in perfect correlation and a slope of 1 . The relationship of sensor 40 to sensor 28 is positive with a correlation coefficient of 0.77 . The other two have negative relationships with sensor 28 and slope down, with correlation coefficients of -0.77 and -0.67 .

Two scenarios can affect the quality of reversal correction using correlation. Firstly, if a poor reference is chosen, such as one without strong breathing motion, then reversal detections will be poor. Secondly, this technique can be disrupted by movements, particularly weight shifts. Fig. 5.3 depicts two sensor signals with different polarities, affected by a head turning movement at $t=26$ seconds. Sensor 16 is likely under the upper chest and sensor 25 is likely to the side of the chest or abdomen. Note that the head movement affects both sensors, even though sensor 25 is not very near the head.

Fig. 5.4 depicts the standardized linear regression and resulting correlation coefficient. The extreme values that occur during the movement push the linear regression of sensor 16 with sensor 25 to a positive slope and to a positive correlation. The 


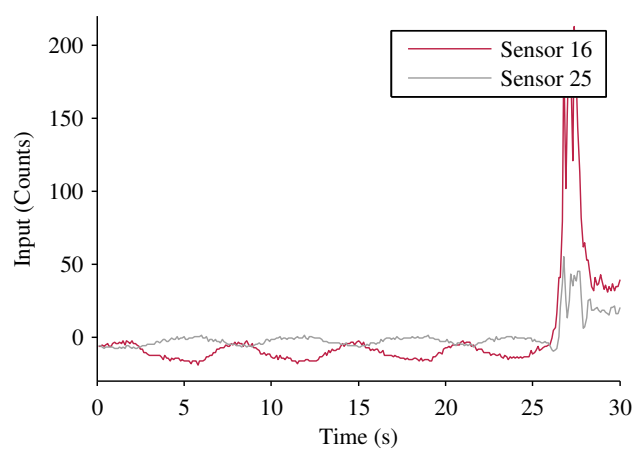

(a) Signals affected by movement

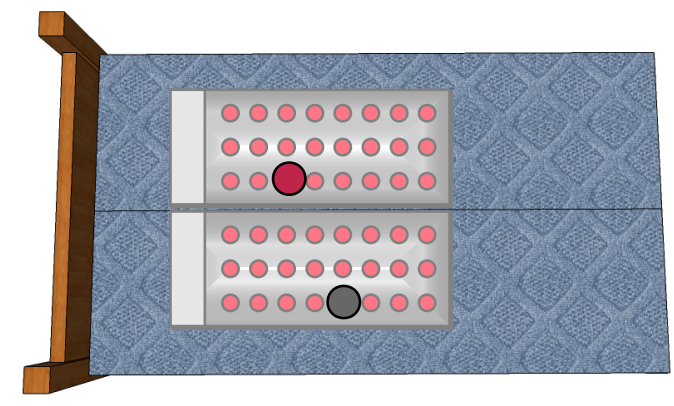

(b) Sensor Locations (red $=$ sensor 16 , grey $=$ sensor 25

Figure 5.3: Sensor signals affected by movement

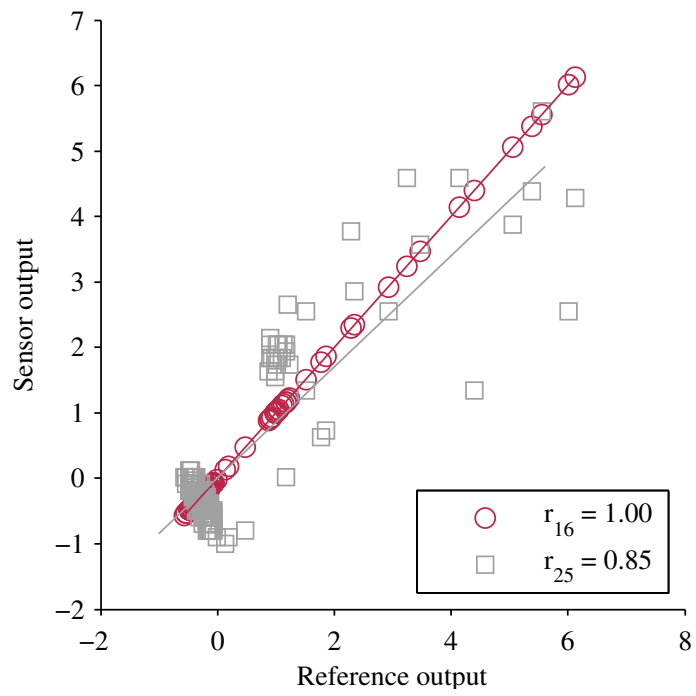

Figure 5.4: Pearson's correlation coefficient as a standardized linear regression during movement 
negative relationship that occurs for the majority of the epoch is only represented by the small cluster near the origin.

The correlation method works well when there is a readily identifiable reference sensor and the subject is breathing quietly. With well-detected movement artefact, corrupted samples could be ignored and would not affect the reversal detection. However, not all movement samples may be detected and other interference such as noise and drift can also obstruct correlation analysis.

\subsubsection{Decision Directed Slope Reversal Detection}

A strategy is considered for multisensor systems to detecting reversals by analyzing the trends in short sliding windows of data. Furthermore, to eliminate reference sensor selection error and make use of the redundant information on the sensors, the reference signal is constructed by fusing sensor signals rather than selecting a single sensor. Fusion is performed using a decision-directed method and the method is labeled as "decision directed slope reversal detection" (DDSRD). Decision-directed techniques have met with success in multi-input applications where ground truth is unknown [162] and are used in a variety of applications including telecommunications [162], [163], voice detection [164], and air traffic control [165].

DDSRD uses the slope of the sensor inputs and the reference to indicate whether a signal is rising or falling. It is helpful that by working with slopes, differing baseline DC values can be ignored and $\mathbf{x}_{\mathbf{r}}$ need not be constructed since only the sign of its slope is required. In this manner, the magnitudes of $\mathbf{x}_{\mathbf{r}}$ and $\mathbf{x}_{\mathbf{k}}$ are never directly compared, providing better immunity to drift, short-term interference bursts and varying signal power. With noisy data, estimating slope using a short window of data rather than the derivative provides a smoothed trend. Fig. 5.5 depicts a block and flow diagram of the DDSRD method, which iteratively refines $p_{k}$ by calculating the trends, updating the reference, and correcting signal polarities. 


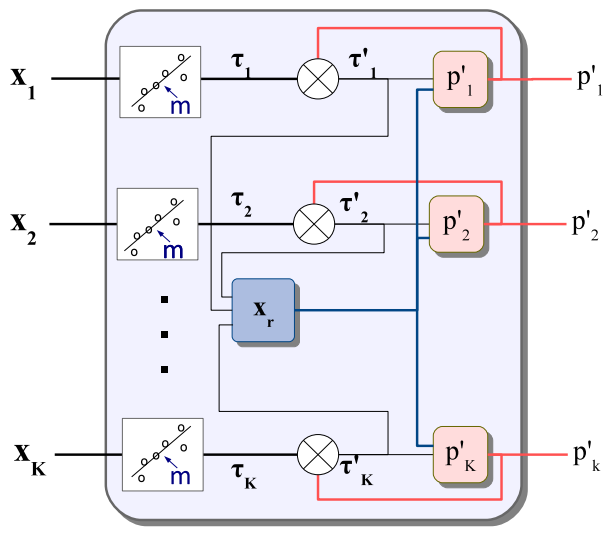

(a) Block Diagram

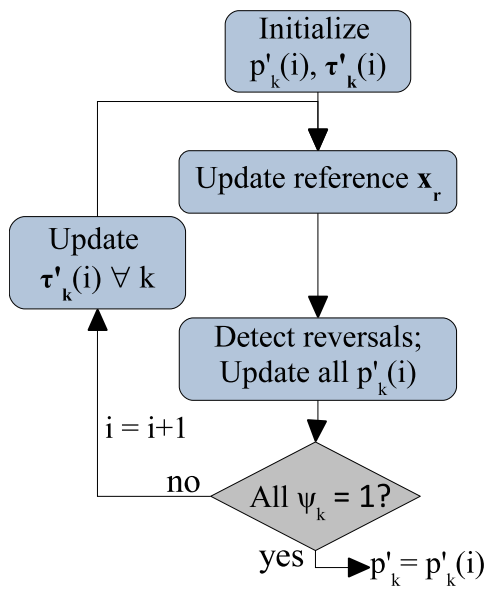

(b) Flow Chart

Figure 5.5: The DDSRD method uses the linear trends $\tau_{k}\left(t_{n}\right)$ to iteratively refine $p_{k}$

\section{Efficient Short-Term Linear Fit}

A local trend $\tau\left(t_{n}\right)$ is calculated at every sample $n$ from a linear regression of a sliding data window of length $R$, centered around $n$. Let $j=[0 \ldots R]$ to indicate the sample numbers in the window. Let $b(j, n)=x(t-R / 2+j)$ represent the data in the window and let $a(j)=\frac{j-C_{1}}{C_{2}}$ represent sample numbers normalized by $C_{1}$ and $C_{2}$ to zero mean and unitary standard deviation, with $C_{1}=R / 2$ and $C_{2}=\sum_{j}\left(j-C_{1}\right)^{2}$. The simple linear regression is formed as follows:

$$
\tau\left(t_{n}\right)=\frac{\sum_{j=0}^{R-1}(a(j)-\bar{a})\left(b(j, n)-\bar{b}\left(t_{n}\right)\right)}{\sum(a(j)-\bar{a})^{2}} .
$$

Normalized $a(j)$ reduces the regression to:

$$
\tau\left(t_{n}\right)=\sum_{j=0}^{R-1} a(j)\left(b(j, n)-\bar{b}\left(t_{n}\right)\right) .
$$


For $t<R$, the signal is prepadded and for $t>N-R$ the signal is padded by mirroring the beginning and end samples respectively. A final step normalizes the slope to the standard deviation of the data in the window, so that areas without a strong trend produce low slopes, irrespective of the signal power:

$$
\bar{\tau}\left(t_{n}\right)=\frac{\tau\left(t_{n}\right)}{\sqrt{\left(\overline{b\left(j, t_{n}\right)}\right)^{2}-\overline{b\left(j, t_{n}\right)^{2}}}} .
$$

The standard deviation can be realized by square route of the windowed variance.

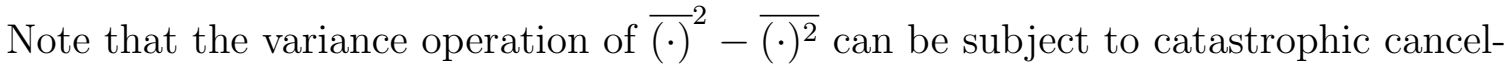
lation in fixed point arithmetic, so care must be taken, but it provides a method of variance calculation that can be efficiently implemented.

The next two steps of reference signal formation and reversal detection are iterated to refine $p_{k}^{\prime}$, using $\bar{\tau}_{\mathbf{k}}$.

\section{Reversal Detection}

Let $i$ be the current iteration and $p_{k}^{\prime}(i)$ be the polarity estimate used during that iteration. Corrected slopes are $\tau_{\mathbf{k}}^{\prime}(\mathbf{i})=p_{k}^{\prime}(i) \bar{\tau}_{\mathbf{k}}$. During the first iteration, $p_{k}^{\prime}(i)$ is initialized to 1 (no correction), and so $\tau_{\mathbf{k}}^{\prime}(\mathbf{1})=\bar{\tau}_{\mathbf{k}}$. The corrected slopes are summed to produce a reference trend $\tau_{\mathbf{r}}(\mathbf{i})=\sum_{k} \tau_{\mathbf{k}}^{\prime}(\mathbf{i})$. The reference is the signum of this trend:

$$
\mathbf{x}_{\mathbf{r}}(\mathbf{i})=\operatorname{sgn}\left(\tau_{r}\right)=\operatorname{sgn}\left(\sum_{k} \tau_{\mathbf{k}}^{\prime}(\mathbf{i})\right)
$$

This equation is effectively a weighted vote using the corrected slope as the weight, giving more credence to sensors with strong slopes and less to sensors which are not strongly linear at a given $t_{n}$. Since the slopes have been normalized, the higher the slope, the stronger the trend and the less likely that the slope sign is due to interference rather than signal progression. 
The error between the reference slope sign and the sensor's sign is weighted by the local signal slope and corrected by the factor $\psi_{k}(i) \in\{-1,1\}$ :

$$
e_{k}\left(t_{n}, i\right)=x_{r}\left(t_{n}, i\right)\left|\tau_{k}\left(t_{n}\right)\right|-\psi_{k}(i) \tau_{k}^{\prime}\left(t_{n}, i\right)
$$

This weighting once again provides for strongly sloped areas to have more sway than less sloped areas. The $\psi_{k}(i)$ factor is optimized by the binary selection of $\psi_{k}(i)=1$ or $\psi_{k}(i)=-1$, minimizing $\sum_{t_{n}} e_{k}^{2}\left(t_{n}, i\right)$. Finally, $p_{k}^{\prime}$ is updated by the optimal $\psi_{k}$ :

$$
p_{k}^{\prime}(i+1)=\psi_{k}(i) p_{k}^{\prime}(i)
$$

The two steps of reference formation and reversal detection are iterated until detected polarities no longer change between iterations $\left(\psi_{k}(i)=1 ; \forall m\right)$ or an upper limit of iterations is completed. During the first iteration, the collective slope can be quite noisy as it is pulled in each direction by the mismatched sensor polarities. However, as the polarities are solidified by iteration, the collective slope smoothes and better represents the underlying signal.

Fig. 5.6 shows all the calculated slopes and reference trend $\tau_{r}\left(t_{n}\right)$ for the two sensors during the example movement epoch. For the most part, sensor 16 decides

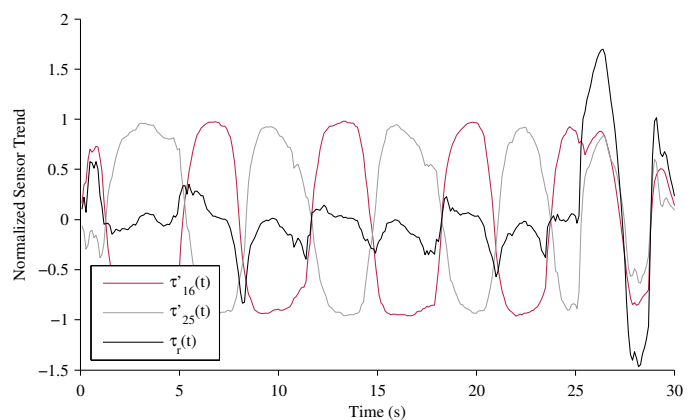

(a) Before Iteration

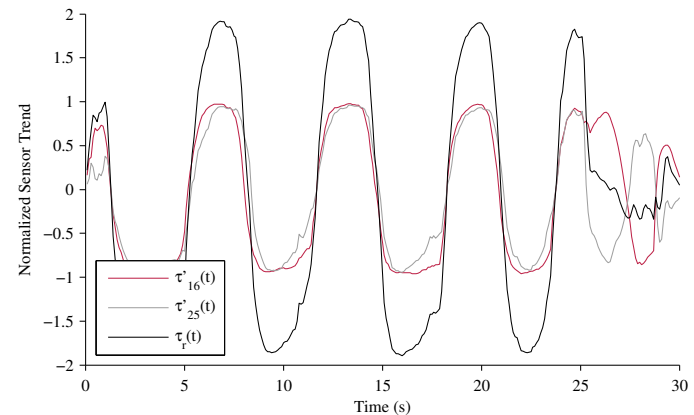

(b) After Iteration 1

Figure 5.6: Normalized slopes and voted direction during movement 
the direction, likely because the noise present in its output is lower with respect to the breathing amplitude.

After the $p_{k}$ is chosen for both sensors, the corrected output is shown in Fig. 5.7. In this example, the reversals are corrected after a single iteration. However, multiple

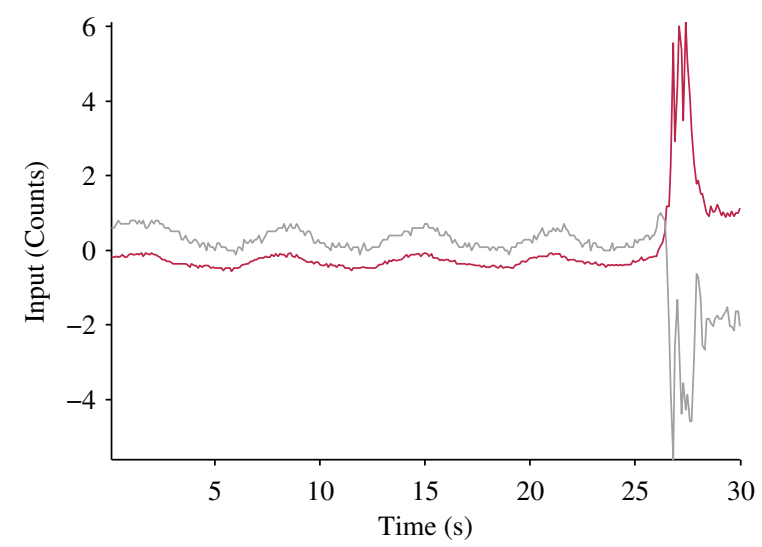

Figure 5.7: Reversals corrected by the short-term DDSRD

iterations are sometimes needed to correct all of the reversals.

Linear fit is a form of correlation to a linear reference [146]. DDSRD can be viewed as a form of correlation analysis where the signals are not compared to each other, but instead to a short-term linear reference.

\subsubsection{Selected Reference Slope Reversal Detection}

To isolate the impact of the fused reference, a method is proposed that follows the same methodology as DDSRD, but selects rather than fuses the reference. For selected reference slope reversal detection (SRSRD), the decision directed portion of DDSRD is replaced by sensor selection, as in the Corr. method. The sign of the selected sensor trend, rather than the decision directed sign, is input to the polarity reversal detection block. 


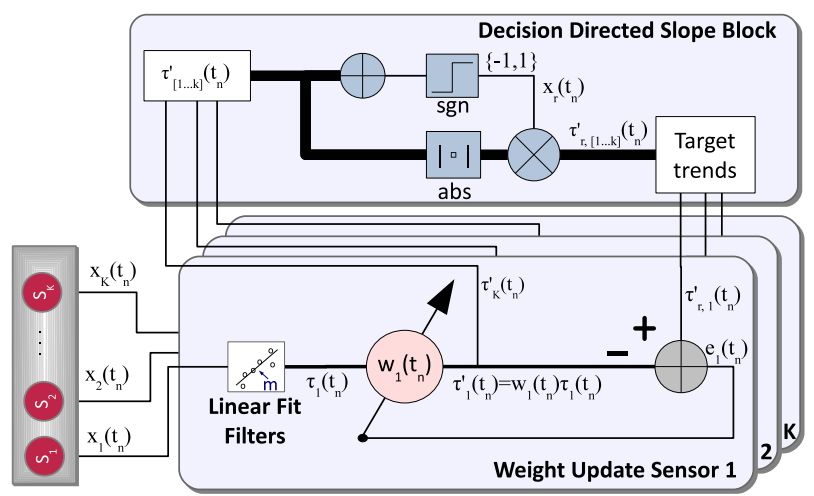

Figure 5.8: Block diagram of online adaptation for polarity weight $w_{1}\left(t_{n}\right)$. A collective slope direction is formed from the sign of the summed weighted input slopes to produce the target slope $t_{1}\left(t_{n}\right)$.

\subsubsection{Adaptive Trend Analysis for Reversal Detection}

Both the correlation method and the proposed short-term trend methods require a full segment of recorded data and are most appropriate for use with pre-recorded data. In this section, the short-term trend methods are modified for efficient online, adaptive processing. The new method is labeled as "Adaptive Reversal Detection" (Adapt RD). A key difference in the adaptive version is that it updates a continuous weight $w_{k}\left(t_{n}\right) \in[-1,1]$, instead of the discrete polarity $p_{k}^{\prime}$. Fig. 5.8 is a block diagram of an adaptive system for DDSRD. For the DDSRD method, the linear fitting of the previous $R$ samples of input can be implemented using two moving average filters: one to perform the fit and one to normalize to the input power.

The input signal trend is multiplied by the current $w_{k}\left(t_{n}\right)$ and fed to the decision directed trend block to form the desired target trend: $\tau_{r, k}\left(t_{n}\right)=x_{r}\left(t_{n}\right)\left|\tau_{k}^{\prime}\left(t_{n}\right)\right|$.

The error is defined as the difference between the target slope and the corrected slope:

$$
\begin{aligned}
e_{k}\left(t_{n}\right) & =\tau_{r, k}\left(t_{n}\right)-\tau_{k}^{\prime}\left(t_{n}\right) \\
& =x_{r}(t, i)\left|\tau_{k}\left(t_{n}\right)\right|-w_{k}\left(t_{n}-1 m i\right) \tau_{k}\left(t_{n}\right) .
\end{aligned}
$$




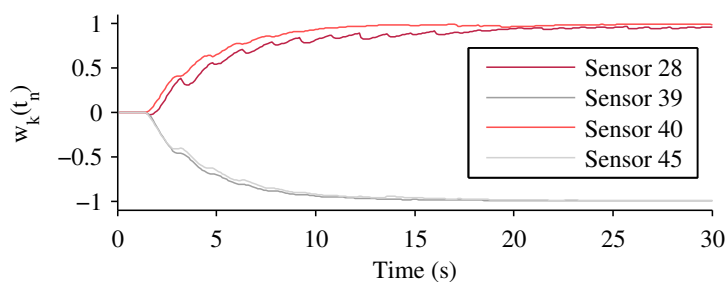

(a) Adaptation of weights $w_{k}\left(t_{n}\right)$

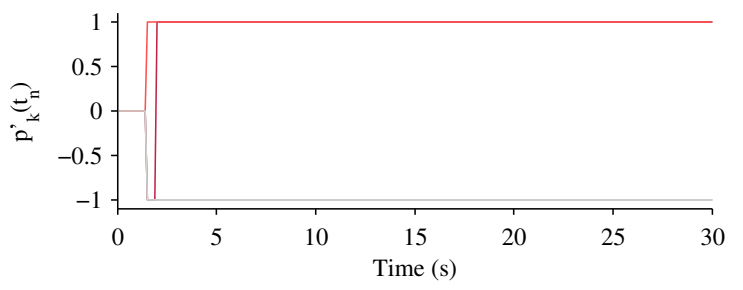

(c) Polarities $p_{k}^{\prime}$

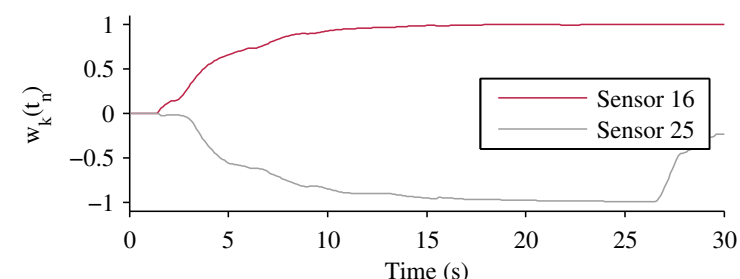

(b) Adaptation of weights $w_{k}\left(t_{n}\right)$ with movement

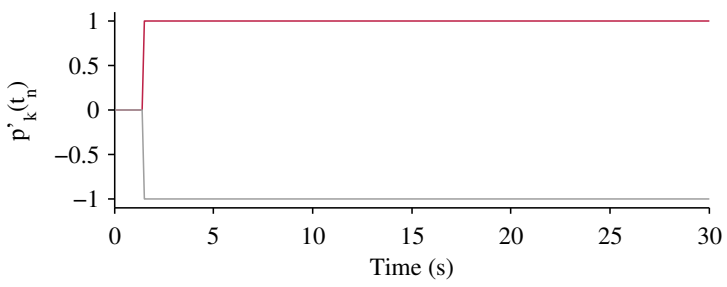

(d) Polarities $p_{k}^{\prime}$ with movement

Figure 5.9: Examples of weight and polarity sign adaptation for the Adapt RD method $(R=15, \mu=0.05)$

This error equation is that same as (5.8), but with a continuous update weight instead of the discrete polarity. Least mean squares is used to adapt the weight [166], parameterized by $\mu$, the step size parameter:

$$
w_{k}\left(t_{n}\right)=w_{k}\left(t_{n}-1\right)+\mu e_{k}\left(t_{n}\right) \tau_{k}\left(t_{n}\right) .
$$

After applying (5.12) to update $w_{k}\left(t_{n}\right)$, any weights outside the $[-1,1]$ interval are reset to a minimum of -1 and maximum of 1 . Polarities $p_{k}^{\prime}$ are the signs of $w_{k}\left(t_{n}\right)$ : $p_{k}^{\prime}=\operatorname{sgn}\left(w_{k}\left(t_{n}\right)\right)$.

This adaptive method can be used online for continuous polarity adjustment or for segment-based processing by passing the segment or a sub-segment through the adaptive analysis. For segment-based processing, $p_{k}^{\prime}$ is set as the sign of the final updated weight at the last sample $n=N: p_{k}^{\prime}=\operatorname{sgn}\left(w_{k}\left(t_{N}\right)\right)$. Fig. 5.9 exemplifies the adaptive process for both the clean and movement examples using the input signals shown in Fig. 5.1 and Fig. 5.3, respectively. The movement occurring at the end of a 


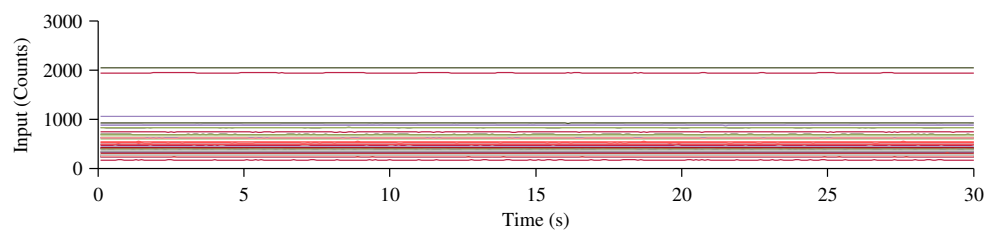

(a) Detrended signals

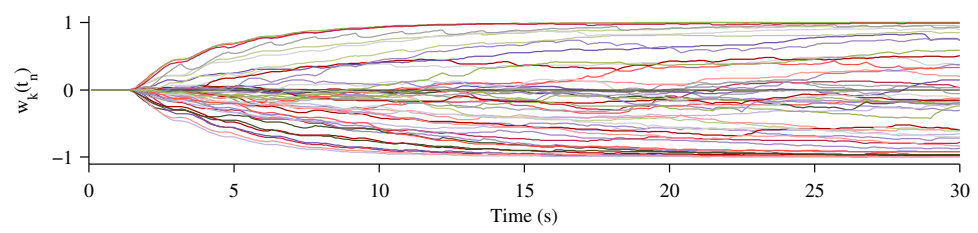

(b) Adaption of weights $w_{k}\left(t_{n}\right)$

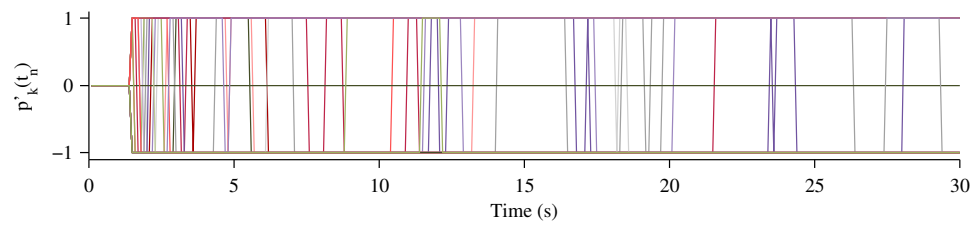

(c) Polarities $p_{k}^{\prime}$ from the adapted weights

Figure 5.10: Large array example adaptation, including noisy signals and the $p_{k}=0$ case on some sensors

segment is the worst case scenario for this method. In the example, the polarities do not change, but the weight of sensor 25 is affected. A longer movement could affect the output polarity.

When Adapt RD is applied to signals from a 48-sensor array, with noisy signals and including the $p_{k}=0$ case on some sensor, an adaptation like the one shown in Fig. 5.10 occurs. This is not as clean cut and shows that many of the sensors constantly change $p_{k}^{\prime}$. However, a core group of signals converge quickly and do not change. These sensors are more likely to hold valid breathing signals.

\subsection{Experiment \#1: Simulation}

The algorithms were first analyzed using simulated signals, to explore their response to generic stimuli. By using the generic stimuli, the effects of specific corruptions could 
be investigated without the confounding effect of multiple sources of interference that occur in experimental data. Parameter selection was first examined, and then a thorough analysis of the effects of interference was undertaken.

\subsubsection{Testing Protocol}

Computer simulated datasets were constructed to model the effects of interference, non-stationary signal periods, and non-linearity. Data segments were created using the signal model in (5.1), starting from a unique zero mean, unitary power base signal $z\left(t_{n}\right)$, with a randomly chosen initial phase. A triangle wave superimposed on a sinusoid and normalized to unitary power was constructed to produce $z\left(t_{n}\right)$. The average period $T$ was jittered by $\mathrm{J} \%$ from period to period to produce a quasiperiodic signal. To model the response of multiple sensors, $\mathbf{z}$ was scaled by a signal-to-noise ratio (SNR) parameter and then replicated $K$ times to create signals $\mathbf{z}_{\mathbf{k}}$ for $k \in(1, K)$. $20 \%$ of the replicated signals were set to 0 to represent $p_{k}=0$ and $40 \%$ of the remaining were reversed by multiplying the scaled $\mathbf{z}_{\mathbf{k}}$ by $p_{k}=-1$. Varying these

proportions was not found to significantly alter results. Unique interference and baseline DC values were added to each $\mathbf{z}_{\mathbf{k}}$ to form signal vectors $\mathbf{x}_{\mathbf{k}}$.

Interference consisted of three components: a noise signal, simulated as AWGN with a constant power; a slow-moving drift signal, whose power was parameterized by a desired signal power to drift power ratio (SDR); and burst interference, parameterized by burst length $L_{b}$. Drift consisted of AWGN, lowpass filtered with cutoff $w_{c}=0.044 \mathrm{rad} /$ sample $\left(f_{c}=0.07 \mathrm{~Hz}\right.$ at sampling frequency $f_{s}=10$ samples $/$ second $)$. Burst interference consisted of a short burst of AWGN noise starting within the segment at a randomly chosen sample and lasting for $L_{b}$ samples. Its power was scaled according to $L_{b}$ and SNR. These interference signals and baseline values were added to clean signals. Quantization noise was included by rounding the resulting signal values to the closest integer. 
A single data set comprised 750 unique data segments, and each segment consisted of 300 samples $\left(N_{s}=300\right)$ from 25 sensors $(K=25)$. With $K=25$, there were 12 $p_{k}=1,5 p_{k}=0$, and $8 p_{k}=-1$. Four main datasets were created, in which subsets were created by varying each of the parameters of SNR, SDR, $L_{b}, J \%$, and $T$, in isolation under conditions of a given noise and drift power. Sets labeled "low noise, low drift" had an SNR of 10 (10 dB) and SDR of 400 (26 dB); "high noise, low drift" had an SNR of 2 (3 dB) and SDR of 400 (26 dB); "low noise, high drift" had an SNR of $10(10 \mathrm{~dB})$ and SDR of $2(3 \mathrm{~dB})$; and "very high noise, very high drift" had both an SNR and an SDR of $1(0 \mathrm{~dB})$. Defaults for the other parameters were $L_{b}=0$, $\mathrm{J} \%=5, T=40$, and $\sigma_{N L}=0$.

Examples of varying the parameters are shown in Fig. 5.11 using the "low noise, low drift" set. Note that the displayed signals are from separate segments and may not share the same initial phase.

Reversal detection performance was assessed by detection accuracy. Accuracy was defined as the percent of correct polarities, where $p_{k}=p_{k}^{\prime}$. Since $p_{k}=0$ was not detected, sensors with $p_{k}=0$ were ignored. For segments with accuracy less than $50 \%, C=-1$ was assumed, and correct polarities for those segments were $p_{k}=-p_{k}^{\prime}$. Thus, the minimum attainable accuracy was $50 \%$.

\subsubsection{Parameter Selection}

The short-term trend methods all require a window length $R$ to be chosen and the adaptive methods have to consider the additional $\mu$ step size.

\section{Window Length $R$}

Fig. 5.12 shows the effect of the window length parameter, $R$, in the midst of drift and noise. All of the methods were affected by noise and drift to some extent. Noise 


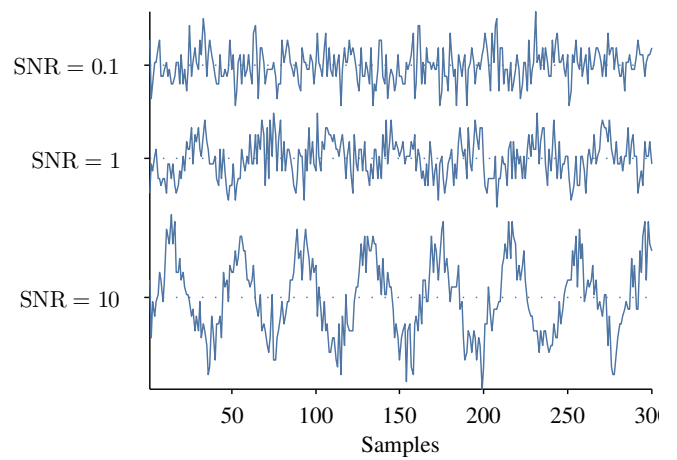

(a) Varying SNR (noise)

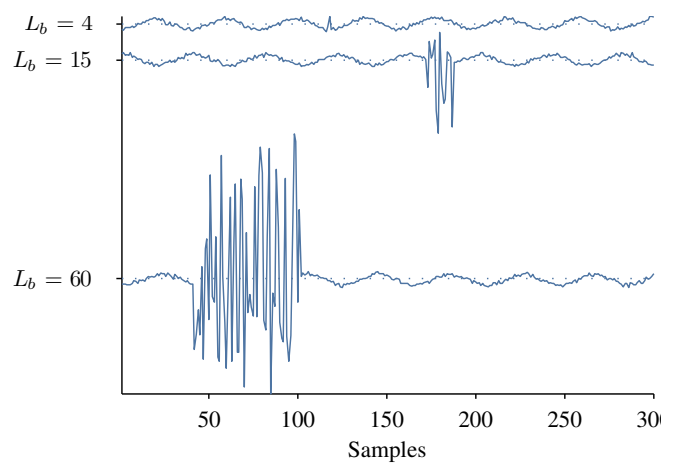

(c) Varying $L_{b}$ (burst interference)

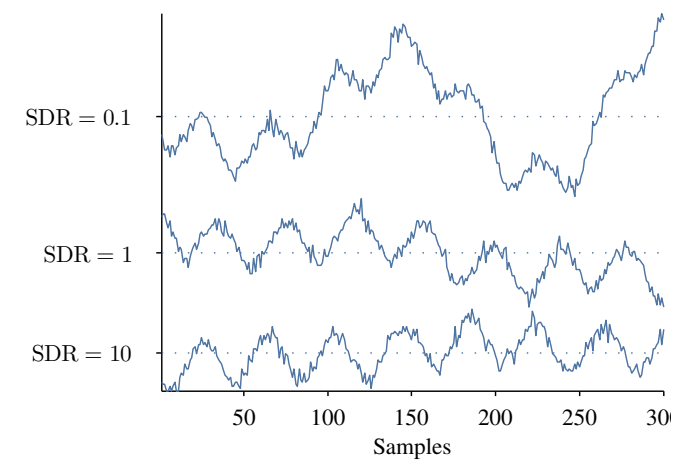

(b) Varying SDR (drift)

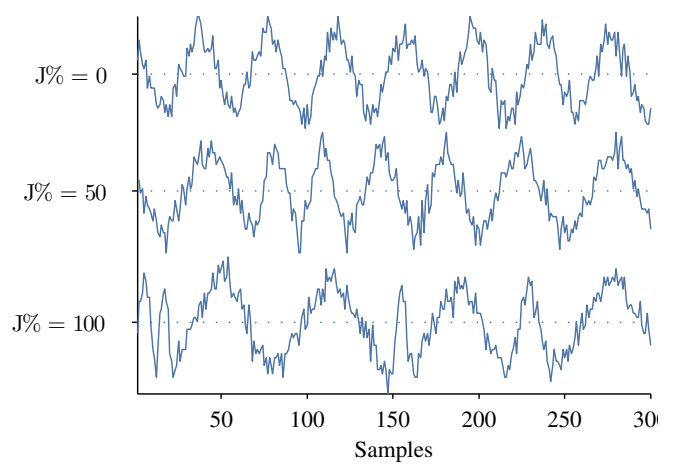

(d) Varying J\% (signal period jitter)

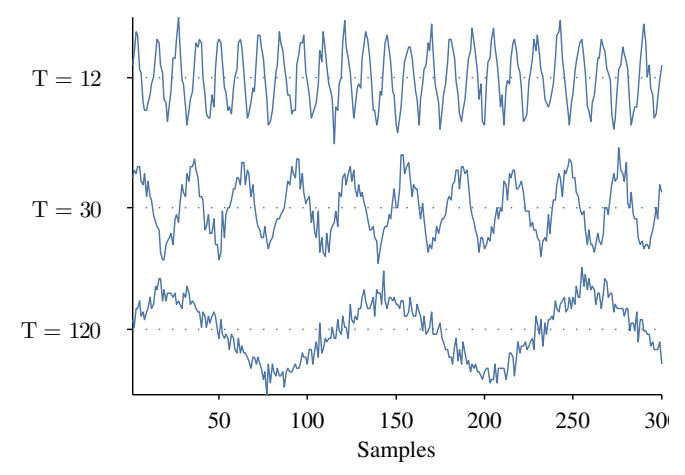

(e) Varying $\mathrm{T}$ (period)

Figure 5.11: Simulated signals with varying parameters 


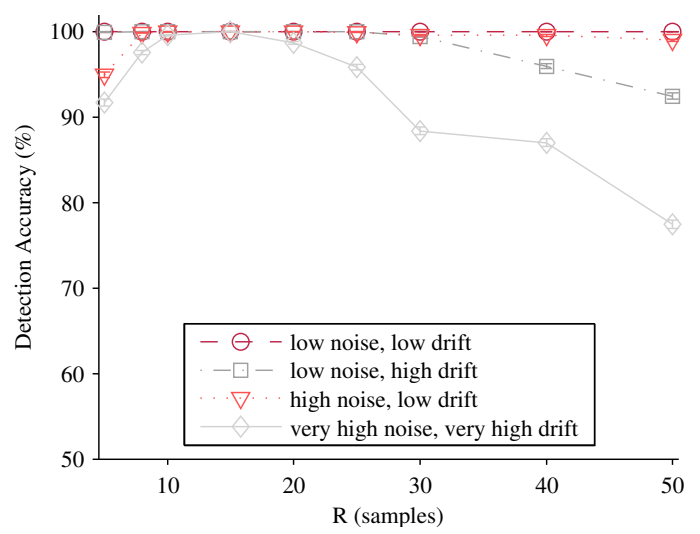

(a) DDSRD

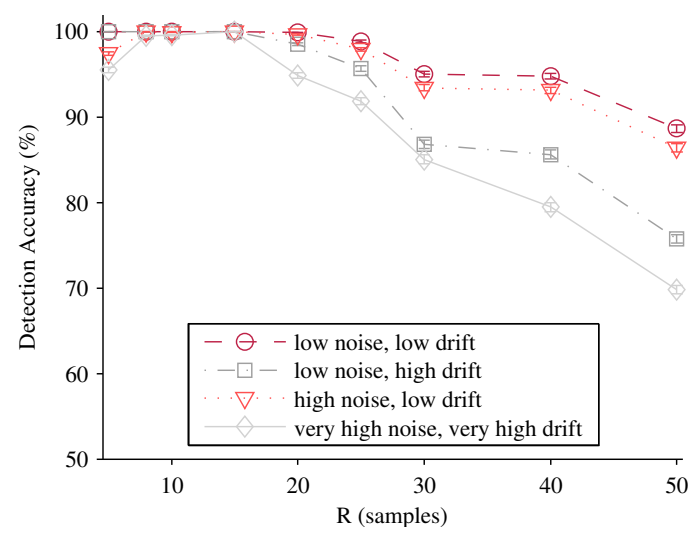

(b) Adaptive DDSRD

Figure 5.12: Accuracy as a function of window length parameter $\mathrm{R}$ in the presence of noise and/or drift

had the effect of decreasing performance for short window lengths, but had less effect at higher $R$. Drift had the opposite effect as it hampered performance for the longer window lengths. When drift and noise were combined, the best choices of $R$ were between 10 and 20 samples. The adaptive variants were less sensitive to noise at short window lengths, perhaps because of the effect of the learning rate smoothing out the noise. Pre-conditioning by lowpass filtering could fulfill this same function for the non-adaptive versions.

The period of the underlying signal is envisaged to affect the optimal value of R. Fig. 5.13 shows the effect of the rate on the optimal choice of $\mathrm{R}$ for the DDSRD method in strong noise and drift. As the window length increased past the signal period, accuracy dropped. For $R>T / 2$, every window includes both rising and falling data, and the slope sign depends on the relative amplitude of the rise versus the fall. Where noise or drift interference was strong enough to overshadow the difference between these amplitudes, errors would have occurred. As $R$ increased, decisions either way were due mainly to the noise or drift. The adaptive methods showed the same effect under the period change. 


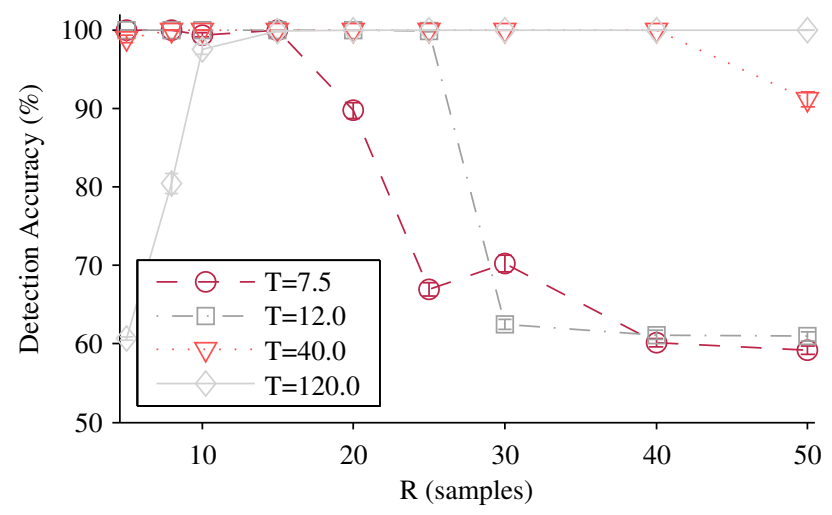

Figure 5.13: Accuracy of polarity reversal detection as a function of window length parameter $R$, varying the period of the underlying signal in strong noise and drift

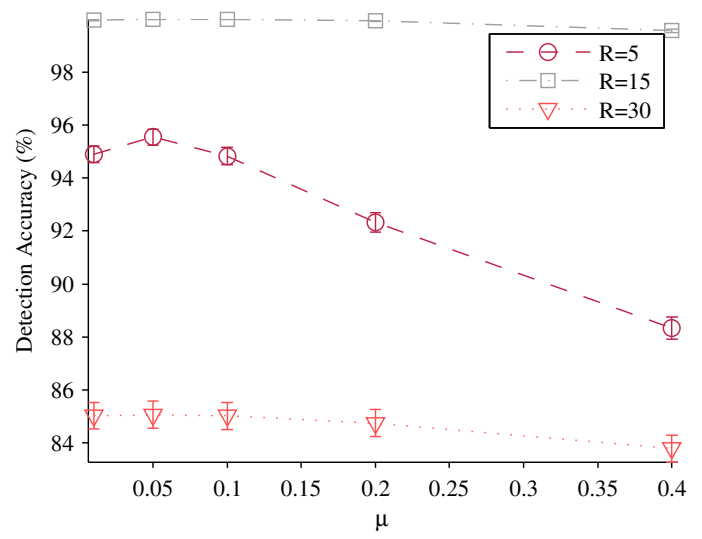

Figure 5.14: Reversal detection accuracy respibands as a function of learning rate $\mu$, varying window length $R$

\section{Learning Rate $\mu$}

For the adaptive methods, the learning rate parameter $\mu$ must also be chosen. Fig. 5.14 shows the effect of different learning rates on the reversal detection accuracy, varying $R$. There is a falloff in performance when the learning rate is too fast ( $\mu$ is too big), with better values less than 0.1. Analysis of the performance of the algorithms for varied signal periods showed that the period did not have a discernible effect on the choice of learning rate for either algorithm.

The best window lengths and learning rate selected for both algorithms were $R=15$ and $\mu=0.05$ respectively. 


\subsubsection{Simulation Analysis}

With parameters of $R=15, \mu=0.05$, and a maximum iteration limit of 10 , the methods were run with simulated data to analyze the effect of three factors: interference, fused reference signals, and non-stationary signal periods. Comparison was also made to the cross-spectral phase (Spec. Phase) method.

\section{Interference}

The effects of interference were assessed for each of the methods. Fig.5.15 shows the effects of AWGN noise on the signals in the presence of low and high drift powers. The

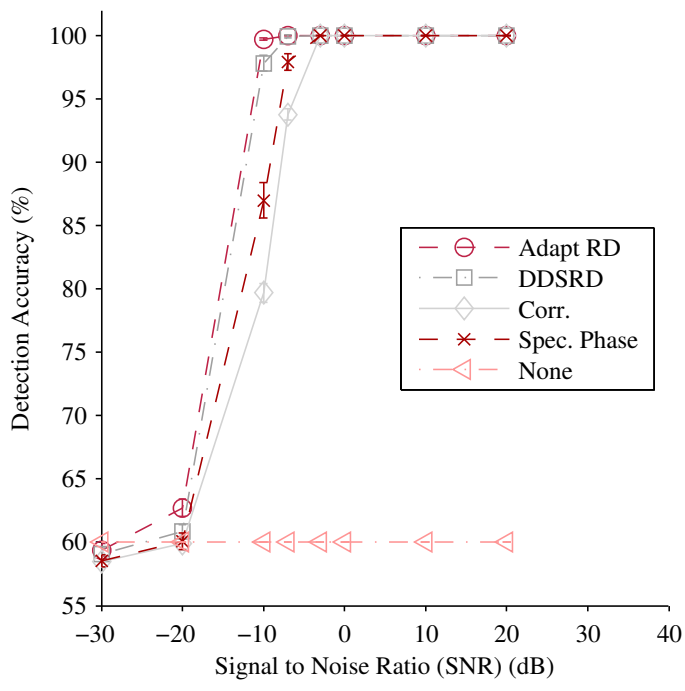

(a) Low drift power

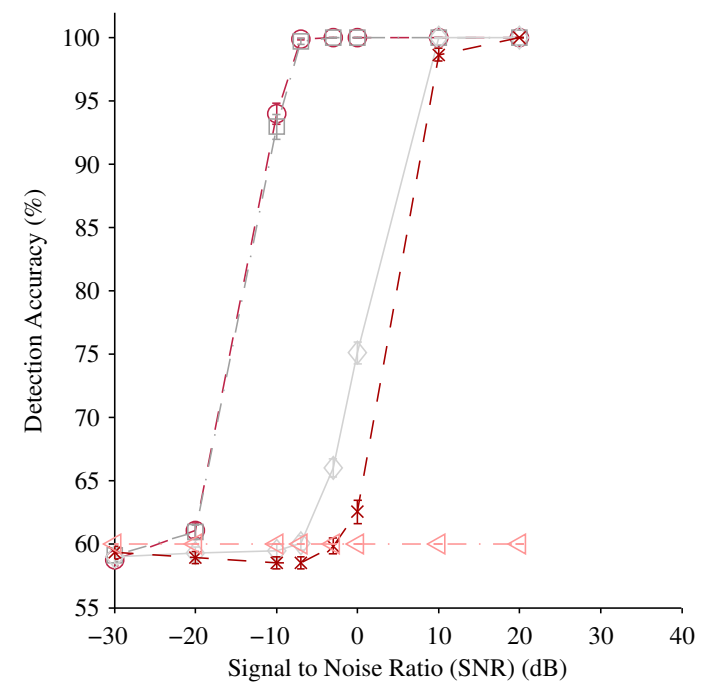

(b) High drift power

Figure 5.15: Effects of SNR on mean percentage of correct polarities: (a) Low Drift (b) High Drift

deleterious effect of noise is amplified by the high drift. The Corr. and Spec. Phase methods are particularly affected by this interference. Even at an SNR of $10 \mathrm{~dB}$, their performance starts to drop. The same performance drop is seen, but at lower values of SNR, for the short-term trend methods. Once SNR reaches -20dB, none of the algorithms perform any better than no reversal detection. In fact, some perform 
slightly worse.

The effect of drift and burst interferences are shown in Fig. 5.16, in conditions of low noise power and for the burst interference, low drift power. The short-term trend

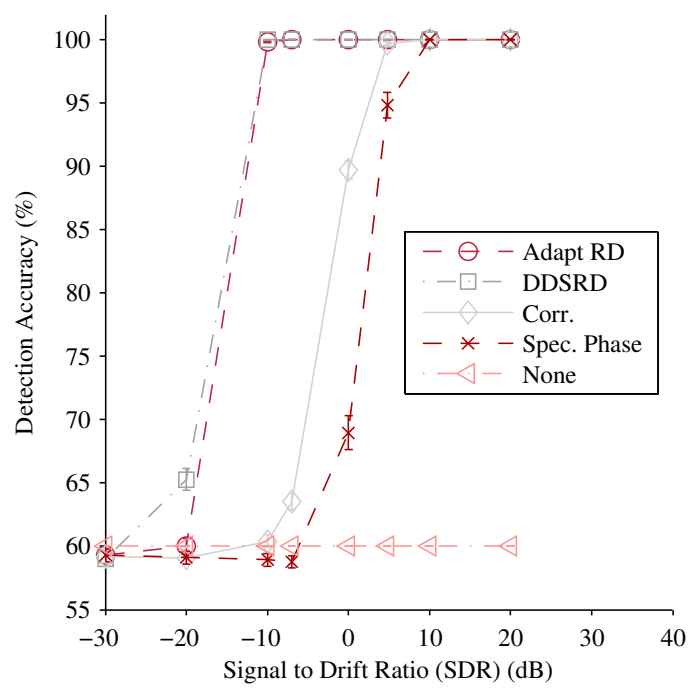

(a) Effect of drift with low noise power

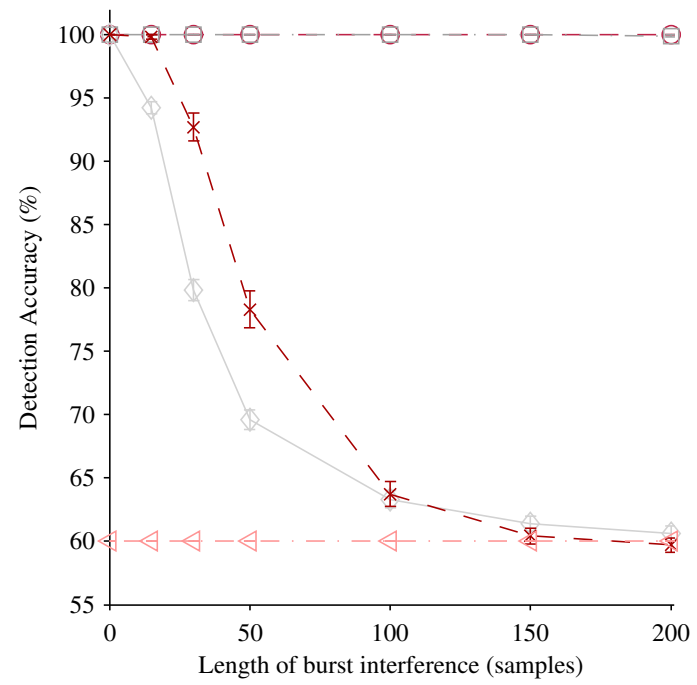

(b) Effect of burst interference with low noise and low drift

Figure 5.16: Effects of interference on mean percentage of correct polarities: (a) Drift (b) Burst Interference

methods were quite resistant to both drift and burst interference. Drift likely affects the Spec. Phase method due to spectral leakage obscuring the frequency estimation and phase discrimination. The correlation method is affected by drift once the power of the drift exceeds the signal power. As posited in Sec. 5.3.1, the correlation method did perform poorly in the presence of movement. The Spec. Phase method was also affected by burst interference to a slightly lesser degree. In high drift and noise conditions, similar results were observed, albeit with a quicker rate of degradation, particularly for the correlation method. 


\section{Effect of the Fused Reference During Reversal Detection}

In comparing the effect of fusion and selection for reference formation, the effects of noise, drift, and burst are revisited for the SRSRD method. The top plots in Fig. 5.17 show the effects of these interference sources on SRSRD, with the DDSRD and Spec. Phase methods shown for reference. At the bottom of the figure, the
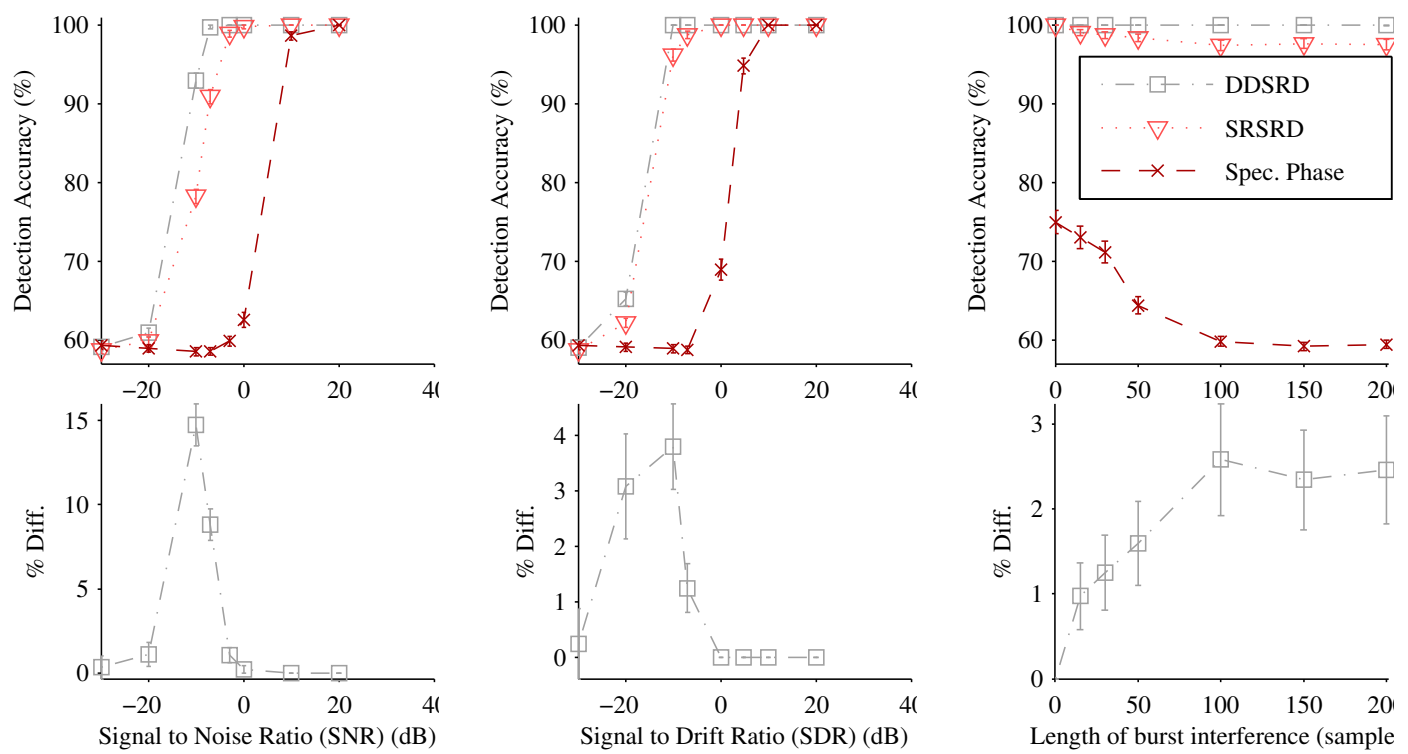

(a) Effect of SNR (high drift(b) power) power

(c) Effect of burst (very high noise and drift powers)

Figure 5.17: Effect of reference fusion on polarity accuracy

performance improvement solely attributable to combining rather than selecting the reference (improvement $=$ DDSRD - SRSRD) is shown. In high drift conditions with varying SNR, the effect of the fused reference is particularly strong, improving the mean detection accuracy by as much as 15\%. The effect of SNR in the low drift condition is not shown, but takes the same shape and ranges up to $6 \%$. The effect of SDR with high noise is also not shown, but is the same as for low noise power. Both the SNR and SDR plots show that the reference provides extra immunity to the effect of these conditions. Similarly, as burst length increases, the effect of the 
reference increases. The effect with burst interference when SNR and SDR are low is negligible.

\section{Effect of Non-Stationary Periods}

When jitter was introduced in higher amounts into period lengths, the Spec. Phase method was affected, as shown in Fig. 5.18. This applied only to low quality input

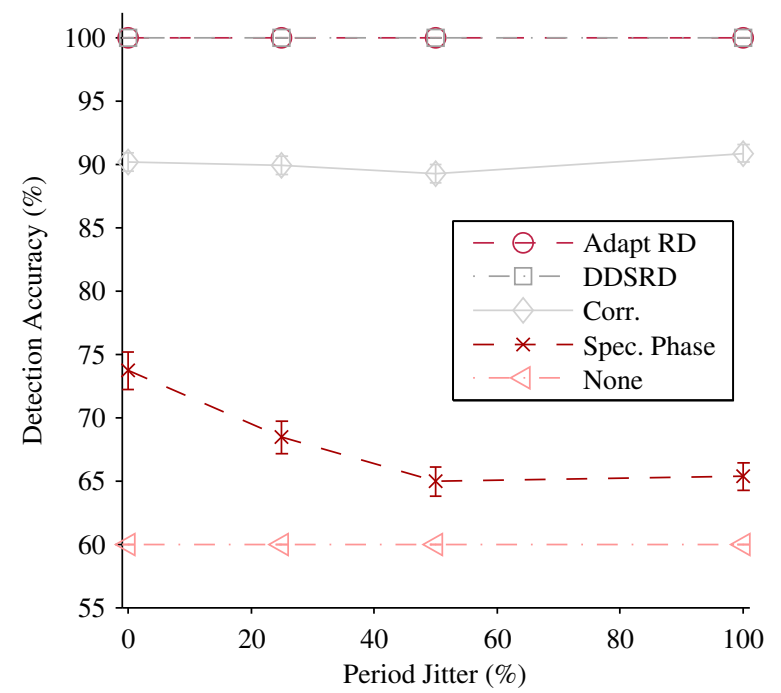

Figure 5.18: Effect of non-stationary periods on accuracy of correct polarities (low SDR, low SNR)

signals, with large noise and drift corruptions. The effect was negligible without those corruptions.

\subsubsection{Discussion}

Noise hampers the performance of all of the analyzed methods of reversal detection, but drift is especially destructive, especially when it is coupled with noisy signals. Motto et al. argued that that noise, changing amplitudes and changing breathing rates would affect the results using cross correlation for asynchrony analysis between thoracic and abdomnial respibands [104]. Indeed, this is supported by the results here 
which show correlation analysis most prone to error when noise or burst interference is present. By using the proposed trend methods, sensitivity to interference was shown to decrease.

Using longer windows for DDSRD was also effective for smoothing the trends to reduce the impact of interference. This is demonstrated by the higher detection accuracy when the window length $R$ is adjusted from a very short window to one with more than ten samples, shown in Fig. 5.12. However, there was a trade-off between immunity to noise and immunity to drift when increasing R. Also, window lengths should not extend beyond the expected period of breathing signals.

DDSRD makes use of a fused reference signal. Wang et al. [112] also used a fused reference signal by iteratively averaging all inputs for EEG spike reversal detection, however the improvement of fusion over reference selection was not addressed. Here, reference fusion outperformed reference selection, particularly at the low signal to interference levels explored in Fig. 5.17. The benefit derived from fusing sensors to create the reference signal is twofold. Firstly, redundant information available across multiple sensors from the underlying signal increases the accuracy of the reference. Secondly, selection between sensors for an appropriate reference is avoided. This process of selection may also be hindered by interference.

The adaptive and block-based DDSRD methods fared similarly in most tests, and a system developer would feel free to select whichever one of these two methods fits their system best. It was shown in the examples of Fig. 5.9 and Fig. 5.10 that the Adapt. DDSRD method could be affected by interference. While individual polarities were certainly affected, this method was less affected than all other methods to the presence of strong noise (Fig. 5.15). It was slightly more affected by drift than the DDRSD method, but less than other methods (Fig. 5.16). It also showed minimal susceptibility to burst interference. 


\subsection{Experiment \#2: Breathing Signal Reversal Detection}

To inspect behaviour of the methods in real-life ambient conditions, experimental results were obtained for bed-based breathing analysis, making use of the data sets outlined in Chapter 3, Sec. 3.3.4.

\subsubsection{Testing Protocol}

Since the interference present in the experimental data may alter the optimal parameters, parameter optimization was applied once again, using the training set. This time, preconditioning by filtering was also considered during optimization, observing results achieved with both lowpass filtered and unfiltered input signals. The lowpass filter had a cutoff frequency of $0.8 \mathrm{~Hz}$, designed as a 128th order finite impulse response (FIR) filter, using the window method with a Hamming window. A FIR filter was chosen rather than an infinite impulse response (IIR) filter, such as Butterworth or Chebyshev, to keep the phase linear and avoid distortion caused by group delay [104].

With selected parameters, comparison was made between reversal detection methods in conditions of rest and movement by processing the clean respiration set and the movement set. Since these data sets are based on the same data as the training data, further testing was pursued using the validation set described in Sec. 3.3.4. The proposed short-term trend methods were again compared to the Corr. and Spec. Phase methods.

Reversals are impractical to manually annotate, especially for small signals that are too noisy to visually assess. The main goal is to provide phase aligned signals that can be summed to provide a respiration signal, and so the methods were evaluated for 
the experimental data by comparing the summed reversal-corrected sensor signals to the respiband standard. Poor reversal correction incurs destructive interference that results in a poorer breathing signal. As detailed in Chapter 3, Sec. 3.4, evaluation of output signal quality was performed using the mean absolute correlation coefficient $(|\bar{r}|)$ between the respiband signal and the summed breathing.

The interquartile ranges of the mean SNR and SDR of the sensor inputs in each epoch were calculated to gauge typical conditions. Breathing signal power was roughly approximated as the mean variance of moving detrended five-second windows. Five seconds was chosen because the longest breathing interval in the normal breathing range of 12 to 20 breaths per minute (BPM) is five seconds. Noise power was approximated as the mean variance of moving, detrended one second windows. Drift power was estimated as the variance of the full epoch less the breathing signal power. SNR was then the breathing signal power divided by the noise power, and SDR was the breathing variance divided by the drift power.

\subsubsection{Results: Parameter Selection}

To choose $R$ for this application, training data were analyzed using varying window lengths, from a half second wide to six seconds wide. Fig. 5.19 shows the correlation of the output respiration to the respibands for DDSRD and Adapt RD methods as a function of $R$. The unfiltered case is also shown for DDSRD, while for adaptive DDSRD, $\mu$ was varied. The results here are similar to those found in simulation. In contrast to the simulation results, there is a strong effect of noise for $R<20$, especially for the unfiltered case. This may be partially attributable to the cardiac pulse BCG which provides interference with a period of 10 samples at 60 BPM. Short movements could also play a role. The optimal $R$ moves up to 20-30 samples (2-3 seconds), rather than between 10 and 20 (1-2 seconds). Lower values of $\mu$ appear to be better here than in simulation, again likely due to smoothing out the cardiac 


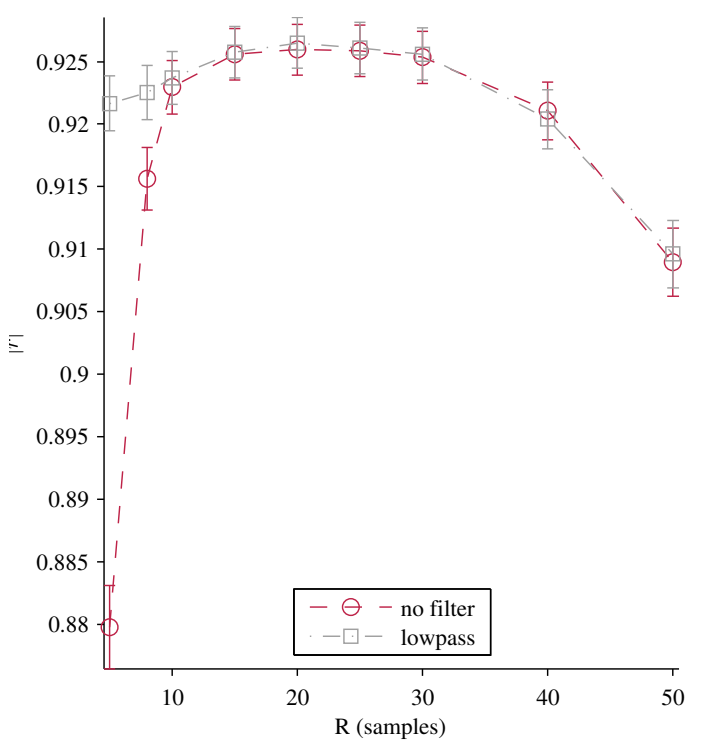

(a) DDSRD

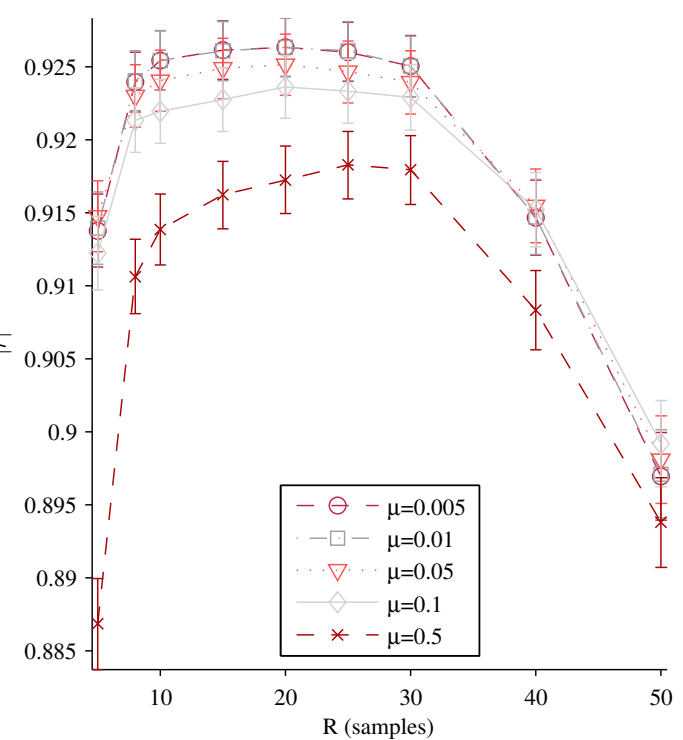

(b) Adaptive DDSRD

Figure 5.19: All experimental training data: accuracy according to window length parameter $R$. Basic and filtered pre-conditioning schemes shown for non-adaptive methods, $\mu$ for the adaptive method.

component in the signal. An $R$ of 20 samples ( 2 seconds) and $\mu$ of 0.01 were selected and lowpass filtering was applied for all subsequent analysis. While the difference between the filtered and unfiltered case was minimal (0.0005), paired Student's $t$ test confirmed it to be significant $(\mathrm{p}=0.019)$.

\subsubsection{Results: Movement Corruption}

Fig. 5.20 shows the effect of movement corruption on the methods using the clean and movement test sets. Epochs were binned by movement length: "none" for the clean set with no movement corruption, "short" for movements less than one second, "mid" for movements between one and two seconds, "long" for movements between two and three seconds, and "very long" for movements between three and four seconds. $80 \%$ of the samples in the test had no movement, and the distribution of the remaining movement-corrupted samples by duration is shown on the right. The numbers of 


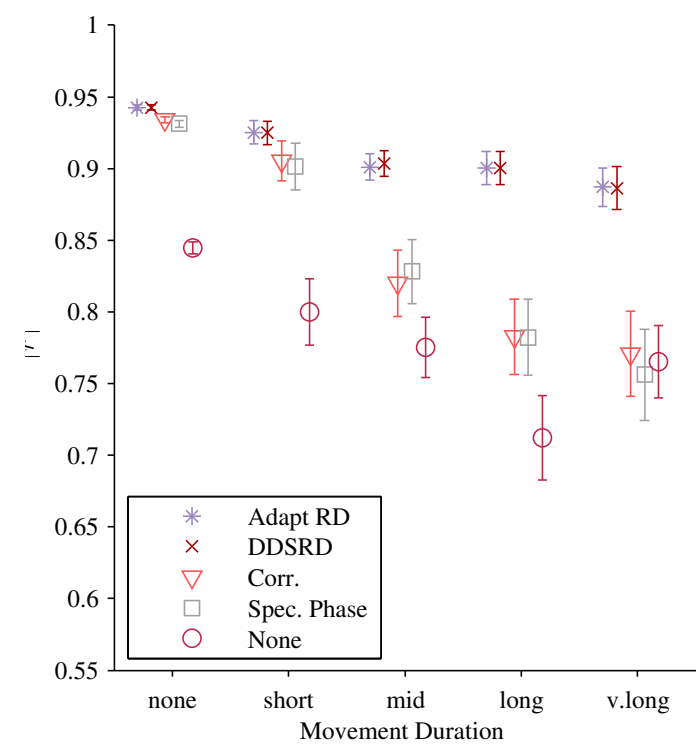

(a) Signal quality degredation due to movement corruption

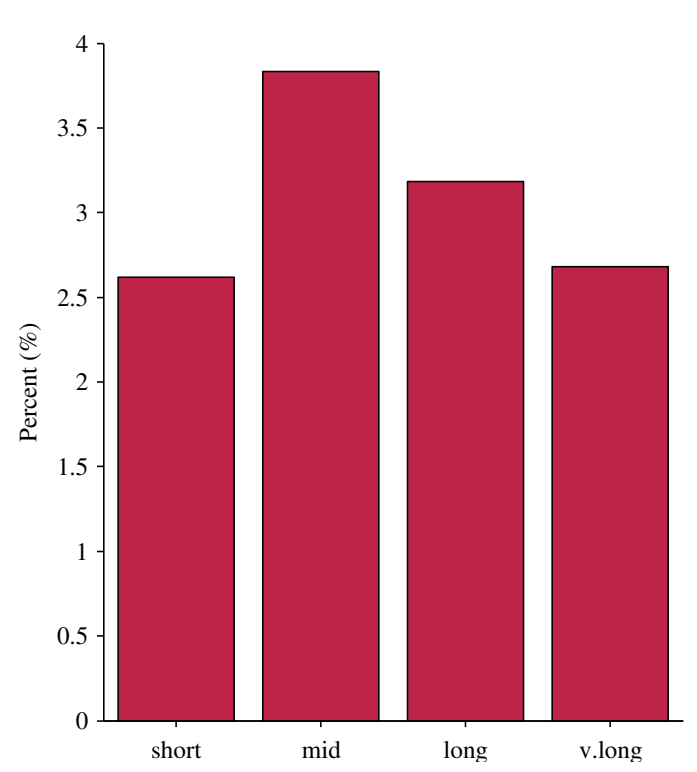

(b) Histogram of movement durations

Figure 5.20: Effect of movement corruption on signal quality

epochs in each bin are relatively equal, although mid length movements are more likely to occur than the others.

As expected, increased movement length caused both the Corr. and Spec. Phase methods to drop more precipitously in signal quality than the short-term trend methods. Notwithstanding the applied method of reversal correction, movement affects the signal quality according to the length of the movement. This can be recognized by the almost linear descent of the signal quality of the "None" method. Although samples with movement artifact are not considered when evaluating the signal quality, some residual effect is still present.

\subsubsection{Results: Validation}

The interquartile ranges of mean SNR and SDR were 2.7 to 9.0 (4.4 to $9.6 \mathrm{~dB}$ ) and 0.8 to 4.5 (-1.0 to $6.6 \mathrm{~dB})$, respectively. Table 5.1 provides test results for the validation set test records and Fig. 5.21 depicts these results through boxplots. The first 
Table 5.1: Performance of reversal correction applied to validation records

\begin{tabular}{|r||c|c|c|c|}
\hline & \% $\mathbb{1}$ & $|\bar{r}|$ & C.I.(low, high) & $r \geq 0.7(\mathbf{\%})$ \\
\hline \hline Adapt RD & 42 & 0.888 & $(0.885,0.890)$ & 90.7 \\
\hline DDSRD & 33 & 0.887 & $(0.885,0.890)$ & 90.7 \\
\hline DDSRD no filter & 33 & 0.885 & $(0.882,0.888)$ & 90.1 \\
\hline SRSRD & 38 & 0.884 & $(0.881,0.886)$ & 89.9 \\
\hline Corr. & 38 & 0.848 & $(0.845,0.852)$ & 83.8 \\
\hline Spec. Phase & 38 & 0.839 & $(0.836,0.843)$ & 81.5 \\
\hline None & 0 & 0.727 & $(0.723,0.731)$ & 63.9 \\
\hline \hline
\end{tabular}

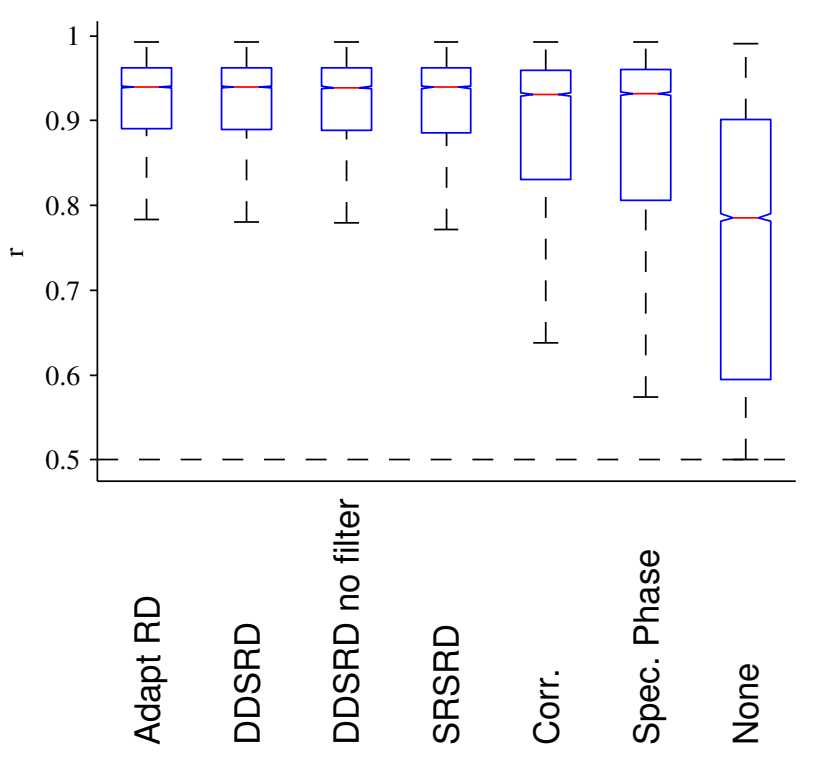

Figure 5.21: Boxplots of output signal quality by method. Median quality is represented by the line across the box, confidence intervals of the median by the extent of the notches, and interquartile ranges by the lengths of the boxes. The extents of the data above and below the first and third quartile are shown by the whiskers. 


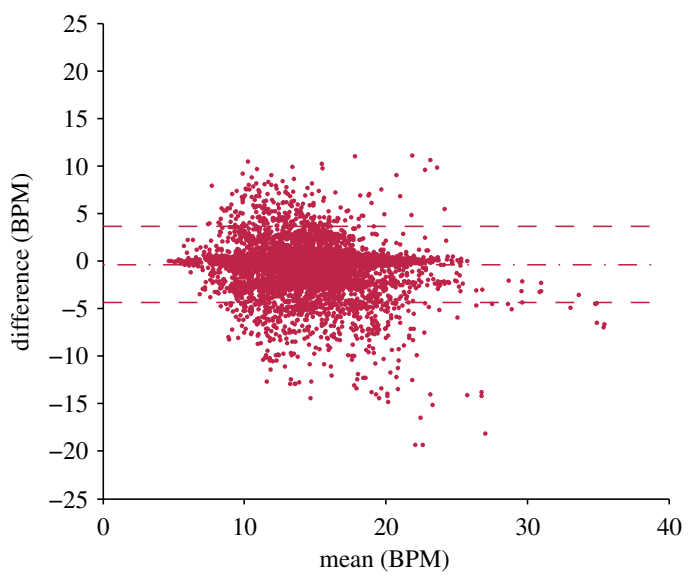

(a) Spec. Phase

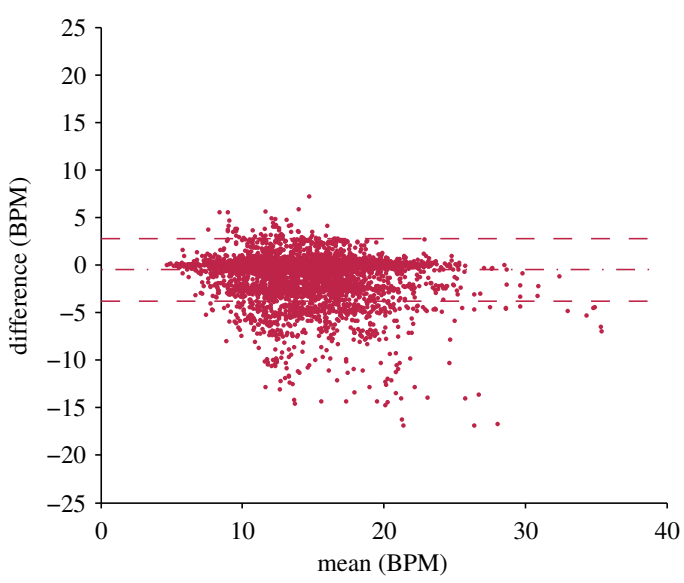

(b) Adapt RD

Figure 5.22: Bland-Altman plots of extracted respiration rates comparing respiband and ambient sensors for Spec. Phase and Adapt RD methods

table column lists the mean percentage of sensor signals that were reversed in each epoch. The number of reversals performed did not seem to correlate with performance. Although their results were very close, and their confidence intervals the same, the improvement of Adapt RD over DDSRD was significant at a $95 \%$ confidence level when paired using Student's right-tailed $t$ test $(p=0.024)$. The paired improvement between the filtered and unfiltered versions of DDSRD, as well as the fused and selected reference signals (DDSRD vs SRSRD) were also significant $(p<=0.001)$. DDSRD and Adapt RD also performed better than the Corr., Spec. Phase methods $(p<=0.001)$.

The validation set shows that the improvements offered by the short-term trend analysis methods during interference translate into gains during real usage. An increase in respiband correlation of over 0.042 was achieved by the short-term trend methods over the Spec. Phase method in this set, resulting in $9 \%$ more output breathing signals with qualities above 0.7 .

Fig. 5.22 depicts Bland-Altman plots for the respiratory rates calculated from the extracted signals. Breathing rates ranged from just below five BPM, when the partic- 


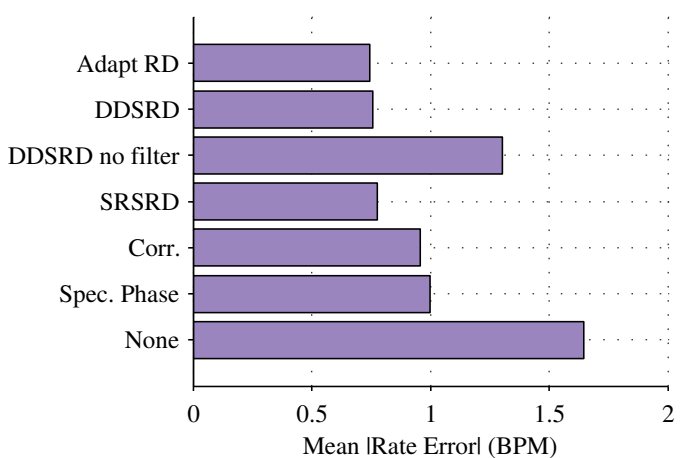

(a) Respiratory Rate Error (BPM)

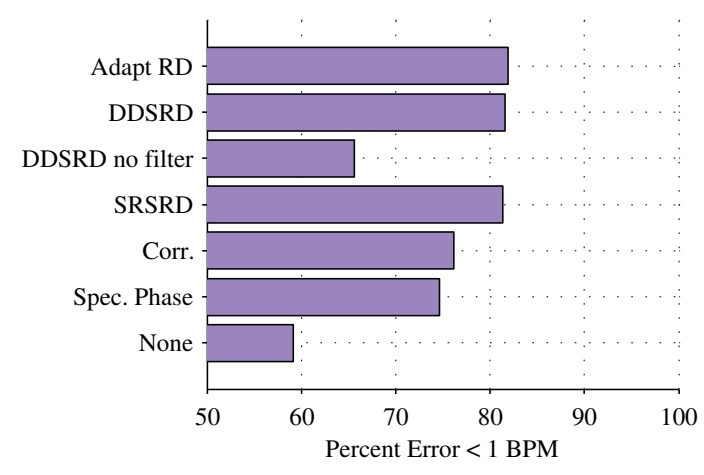

(b) Rate estimates within 1 BPM (\%)

Figure 5.23: Respiratory rate agreement with respibands by reversal detection method

ipants were asked to breathe deeply, to $35 \mathrm{BPM}$ when they breathed shallowly. The Adapt RD method provides a narrower difference at any breathing rate, although it was slightly more likely to overestimate the rate than underestimate it. The resulting mean respiratory rate error and the percentage of estimates within 1 BPM are shown in the barcharts of Fig. 5.23. Mean rate errors for spectral phase were 1.00 BPM, compared to 0.76 for DDSRD. This represents a reduction in rate estimate error of almost $25 \%$, which increased the number of epochs with less than a 1 BPM error from $75 \%$ to $82 \%$. Lowpass filtering has a much larger effect on the respiratory rate error than on the signal quality. Note that all algorithms, except the no-filter DDSRD method, had lowpass filtering applied, so comparison between that method and non-DDSRD algorithms are not valid; the other algorithms would likely also produce higher rate errors without the filtering.

Table 5.2 compares the impact of respiratory rate extraction by participant. Mean breathing rates tended to be slightly on the low side of the expected 12 to 20 BPM range, with two participants just above a median of $12 \mathrm{BPM}$. This is partly accounted for by the fact that the participants were resting quietly. The participants were a strong source of variability in the results, with P49 consistently displaying half the rate error as P95. P95 was the heaviest participant and also the only male in the validation 
Table 5.2: Statistics of respiratory rate estimation after reversal corrections using Adapt RD, listed by participant

\begin{tabular}{|r|c|c|c|c|c|c|}
\hline & P49 & P61 & P82 & P89 & P95 & All \\
\hline \hline Median respiband rate (BPM) & 16.60 & 16.32 & 14.75 & 12.68 & 12.15 & 14.78 \\
\hline Median Adapt RD rate (BPM) & 16.73 & 16.90 & 15.00 & 13.22 & 12.33 & 15.18 \\
\hline \% not available & 0.38 & 0.30 & 0.80 & 0.51 & 1.12 & 0.61 \\
\hline Mean |error| (BPM) & 0.46 & 1.10 & 0.75 & 0.74 & 0.67 & 0.74 \\
\hline Median |error| (\%) & 0.70 & 1.01 & 1.11 & 1.61 & 1.19 & 1.06 \\
\hline Mean |error| (\%) & 3.38 & 8.33 & 5.61 & 6.83 & 6.27 & 6.08 \\
\hline \% |error| $<1$ BPM & 90.99 & 73.44 & 81.29 & 79.08 & 85.02 & 81.95 \\
\hline \hline
\end{tabular}

set. However, the results were not correlated with weight; the two participants with the best results, P49 and P95, were also the second and third heaviest.

\subsubsection{Discussion}

The problem of reversal correction was approached in this thesis in its own right, rather than as a subset of TDE. This approach allowed the problem to be directly addressed as the optimization of the sign of the slope instead of the more oblique delay measurement or spectral phase analysis. The method is waveform independent, robust to interference from various sources, and applicable to periodic, quasiperiodic, and aperiodic data alike. While the linear slope was used here, other trend analysis techniques, such as Cox Stuart trend tests [167], could be applied following a similar methodology. Cox Stuart trend analysis was also trialed with this data and showed similar results to the linear trend DDSRD method. Results were not shown here as they made the plots busier and more difficult to read, without significantly adding to the discovered knowledge. However, in some systems, Cox Stuart trend analysis might be a simpler test to implement. 
The experimental results were consistent with results obtained during simulation. The range of mean SNR and SDR values of the experiment both fell within simulation regions where the DDSRD method differentiated itself from the the Corr. and Spec. Phase methods, although not at that point from the DDSRD method (see 0-10 $\mathrm{dB}$ range in Fig. 5.15 and Fig. 5.16a). This is reflected in the experimental results with a clear gain over the Corr. and Spec. Phase methods, but a closer result to the DDSRD method.

The shape of the parameter optimization curve of the experiment (Fig. 5.19), with a droop over higher values of $R$, suggests the effect of drift: droop occurred as drift was increased during simulation in Fig. 5.12. The effect of noise with very low values of $R$ seen in that simulation was not as pronounced in the experiment due to the lowpass filtering that was applied to preprocess the signals. This lowpass filtering was shown to decrease respiration rate error. Analysis of the effect of lowpass filtering on other algorithms was not done, but could yield interesting results.

In applications with higher drift and noise, it is expected that the DDSRD method could further distinguish itself from Corr., Spec. Phase, and even SRSRD. Drift encountered in other applications would depend on the sensor type being used and the environmental conditions such as temperature fluctuations. Even within the application of bed-based monitoring, higher SDR and SNR values are possible. Slow relaxation while falling asleep could cause a drift-like signal. Furthermore, as seen in Chap. 4, installation below thicker mattresses could affect SNR.

Window lengths that are appreciably less than the shortest expected period of breathing signals have been conventionally used for analysis of breathing. For example, Wilks et al., detected infant breaths with a window length of 0.24 seconds [152], to correspond to one quarter of a 60 BPM infant respiratory cycle. Moyles et al. used window lengths of 1.875 seconds [168] with adult breathing. This length is just over half of the three second period of 20 BPM. Analysis of window lengths with respect 
to period (Fig. 5.13) showed the importance of choosing lengths that are smaller than the period. A window length of 2 seconds (20 samples) was selected here, which is less than half of the 5 second period of 12 BPM and still less than the 3 second period of 20 BPM.

The removal of the filtered components of the interference reduces the impact of noise and interference from signals like the heart rate. There is a statistically significant $(p<0.001)$ advantage to pre-filtering the signals with a lowpass filter for experimental data. This achieves an improvement gain of $2 \%$, enough to justify the incremental increased in complexity.

The signal model introduced in Sec. 5.2 is valid when systemic signal delays between sensors are much less than one half of the fundamental period of the signal. For higher frequency signals with measureable propagation delays, $g_{k}(\cdot)$ includes a phase offset. In addition to reversal correction, signal alignment would also require a matching delay correction. The phase coherence problem then becomes a TDE problem in addition to the reversal problem that is considered here. Townsend et al. provided a method of both reversal and delay estimation using correlation analysis [53]. Similar delay detection could be applied in the context of decision directed reversal detection by secondary correlation analysis of $t_{k}(n)$ and $m_{k}^{\prime}(n)$, although care would be needed in the presence of movement since the correlation method is sensitive to movement. Furthermore, in the previous chapter, it was discovered that non-linearity could masquerade as signal reversals and delays of one quarter period. If delay correction is applied, only short delays less than the quarter period should be considered.

The improvements offered by the short-term trend algorithms in the controlled laboratory setting of the experiment may be be more substantial in care settings. There, thicker or denser mattresses could appreciably lower signal quality, while movement may be accented by more time spent in bed while awake. The improvement by using DDSRD over cross-spectral phase analysis was small but significant in the validation 
data.

The correlation method can be modified to ignore periods of movement by simply dropping samples during detected movement. However, movement detection is not always accurate, and some movements may be missed. The spectral phase method is also vulnerable to movement, particularly since movement also occupies the respiratory spectral band and corrupts the phase estimates at the breathing frequencies. The spectral phase method would be difficult to modify in order to ignore periods of movement. In scenarios where movement is expected to occur frequently, the proposed trend methods offer protection from the effect of undetected movement.

The results highlight the importance of aligning the sensor signals before summing them to extract a respiratory signal. All of the methods of reversal detection and correction provide a marked improvement in the output respiratory signal when compared to not providing any phase correction. However, periods of movement, noise corruption, or reductions in the number of sensors do affect method performance.

Improvement to combining performance may be possible by detection of the $p_{k}=0$ case, setting those sensor inputs to a constant zero. However, although some methods were trialed, they generally resulted in worse combining performance than without this detection. It is desirable that the fusion stage is robust to reduced availability, where the inclusion of the $p_{k}=0$ sensors as $p_{k}= \pm 1$ does not inhibit good fusion.

While the main field of application here is towards coherent combining of sensor inputs, the methods may also be useful in other applications where reversals suggest a latent condition. For example, in biomedical sensing of cardiopulmonary signals, reversals between thoracic and abdominal breathing signals can indicate paradoxical motion caused by diaphragmatic paralysis or obstructive apnea [169]. In this work, it was assumed that all reversals were systemic rather than physiological in nature, but physiological reversals may be possible. Discerning physiological reversals would be of interest to clinical applications, but it is currently not known if this is possible. 


\subsection{Summary}

Detecting signal reversals is an important step for many fusion algorithms. Three methods were proposed to detect reversals from ambient sensors: the correlation coefficient method (Corr.), decision directed slope reversal detection (DDSRD), and an adaptive version of DDSRD (Adapt RD). These all offered improvements over spectral phase analysis in conditions of interference and for non-stationary signals. The proposed correlation coefficient sign method improved reversal detection for nonstationary signals, but was hampered by interference. On the other hand, short-term trend analysis methods of DDSRD and Adapt RD showed resiliency to both conditions of interference and non-stationary behaviour. Compared to spectral phase analysis, this provided $9 \%$ more signal segments with signal qualities above 0.7 , which reduced respiratory rate errors by $24 \%$ and increased the number of segments with errors less than 1 BPM by $7 \%$. 


\section{Chapter 6}

\section{Respiratory Signal Fusion by Linear Combining}

This chapter examines breathing signal fusion as a linear combining scheme, where individual sensor inputs are weighted by a scalar before summation. Three categories of linear combining are emphasized: sensor selection, weighted sensor summation, and blind source separation. These combining techniques were introduced in the background review of signal processing (Chap 2, Sec. 2.2.4) and methods for each are category are developed and proposed in this chapter, Secs. 6.2.2, 6.2.3, and 6.2.4. Emphasis is on conditions of low input signal quality, reduced signal availability, and disorder in the signal periods. To this end, conventional and new methods were compared and contrasted under each of these conditions.

Methods for each of the three fusion categories are proposed. Sec. 6.2.2 offers an improved selection combining method, for situations where selection combining is desirable. This method is specifically designed to be robust to movement and noise and was published in [55] and submitted to [51]. Sec. 6.2.3 investigates applying diversity combining and specifically maximal ratio combining (MRC), towards selecting optimal gain factors. This technique was published in [54]. Blind source separation methods are explored in 6.2.4. A principal component analysis (PCA) method that 
selects the best principal component is proposed, and independent component analysis is also applied with principal component analysis for dimension reduction and selection combining for component selection. In addition, the method of adaptive DDSRD reversal detection developed in the previous chapter (Sec. 5.3.2) is extended to a blind source separation method in Sec. 6.2.4

\subsection{Introduction}

The three fusion techniques of sensor selection, weighted summation, and blind source separation all fit the linear combining paradigm. That is, they make use of the following model for signal fusion:

$$
z^{\prime}\left(t_{n}\right)=\sum_{k=1}^{K} g_{k} x_{k}^{\prime}\left(t_{n}\right)
$$

$x_{k}^{\prime}\left(t_{n}\right)$ represents the input signal, $x_{k}\left(t_{n}\right)$, after it has undergone signal conditioning. $k$ denotes the sensor number. The output signal, $z^{\prime}\left(t_{n}\right)$, is constructed from the sum of the signals, each first multiplied by a gain factor $g_{k}\left(t_{n}\right)$. The gains differ according to the linear combining method that is used, and the gain value, $g_{k}$, can be different on every sensor.

\subsection{Methods}

Before attending to fusion methods, spectral estimations of signal power, noise power, and in-band power from quasiperiodic signals with unknown frequency are addressed. These spectral parameter estimates are used throughout the chapter to support measures of signal strength. With these in hand, fusion methodologies were developed. 


\subsubsection{Spectral Parameter Estimation}

To extract spectral parameters of signal power, noise power, and in-band power, three spectral estimates are first made: power spectral density, fundamental frequency, and noise spectral density. The frequency band of interest for respiratory signals is 0.07 $\mathrm{Hz}$ to $0.8 \mathrm{~Hz}$, corresponding to roughly from four to fifty breaths per minute (BPM).

Mean removal or detrending is an essential preprocessing step, as a DC value creates significant spectral leakage. Once signals are detrended, the PSD is estimated using a modified (windowed) periodogram, zero padding the samples of the epoch/segment to a length that is a higher power of two for spectrum interpolation and fast fourier transform (FFT) efficiency. Windowing widens the respiratory signal lobe in the PSD, but reduces the spectral leakage. Reducing spectral leakage will ensure that noise estimation in subsequent analysis is more accurate. The Hanning window is used here, since it provides a good trade-off between lobe width and spectral leakage.

Spectral parameter estimates are made under the assumption that there exists a peak in the power spectrum that corresponds to the fundamental frequency of the signal of interest. The fundamental frequency is set as the frequency with the highest peak in the PSD within the respiration band.

The noise spectral density is required for both noise power and signal power estimation. Fig. 6.1 shows the PSD of a respiratory signal from one sensor. The main signal lobe is located as centered at the fundamental respiration frequency, with a width given by two times the $3 \mathrm{~dB}$ width of the Hanning window, considering the interpolation width provided by zero padding. Fig. 6.1a highlights the main signal lobe.

Noise is found in the bins within the respiratory range, but outside of the main signal lobe and its possible harmonics. The noise spectral density $N_{0}$ is estimated as 


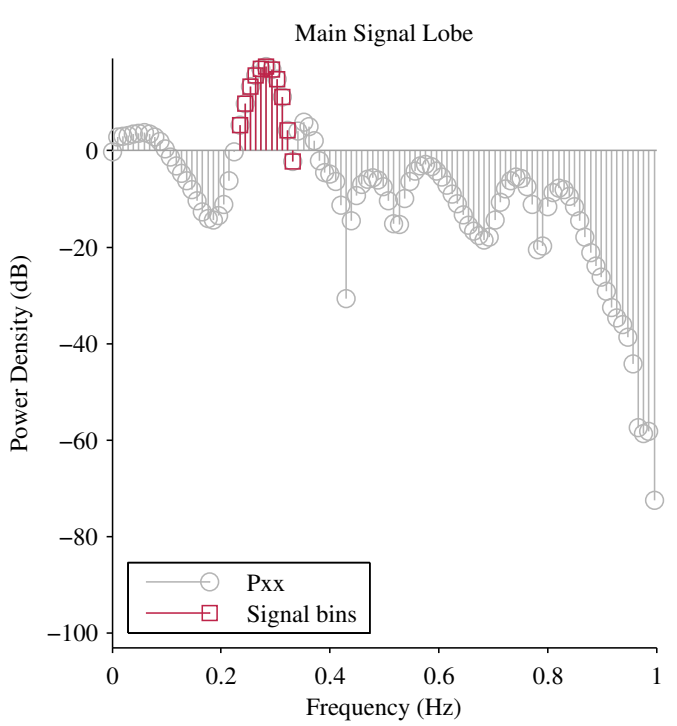

(a) PSD, highlighting the main signal lobe

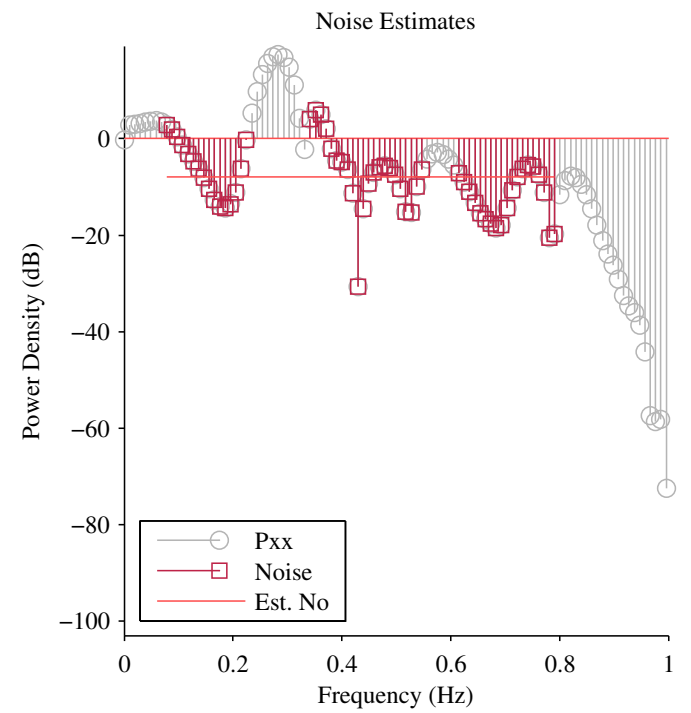

(b) PSD, highlighting the included noise bins and estimated $N_{0}$

Figure 6.1: Locating the signal and the noise components in the PSD

the median value in the noise bins. Using the median, rather than the mean, guards against spectral leakage from the main signal lobe affecting the estimate. Fig. 6.1b highlights the included noise bins and $N_{0}$ value. This estimation of noise spectral density assumes that noise is white (uniform), which does not hold when coloured interference, such as movement, is present. Even so, noise density increases with coloured interference too, and the estimate may serve for many use cases.

Finally, the three spectral parameters of interest can be extracted. To estimate the total noise power $N$, the noise spectral density is integrated across the entire respiratory band. The signal power $S$ is estimated by integrating the power densities of the main signal lobe and subtracting the noise power present in the lobe. The total power in the respiratory band $P_{r}$ is estimated by integrating all in-band power densities (all highlighted bins in Fig.6.1).

The primary assumption made that there exists a single peak in the spectrum belonging to the signal of interest is valid when changes in period within the signal 


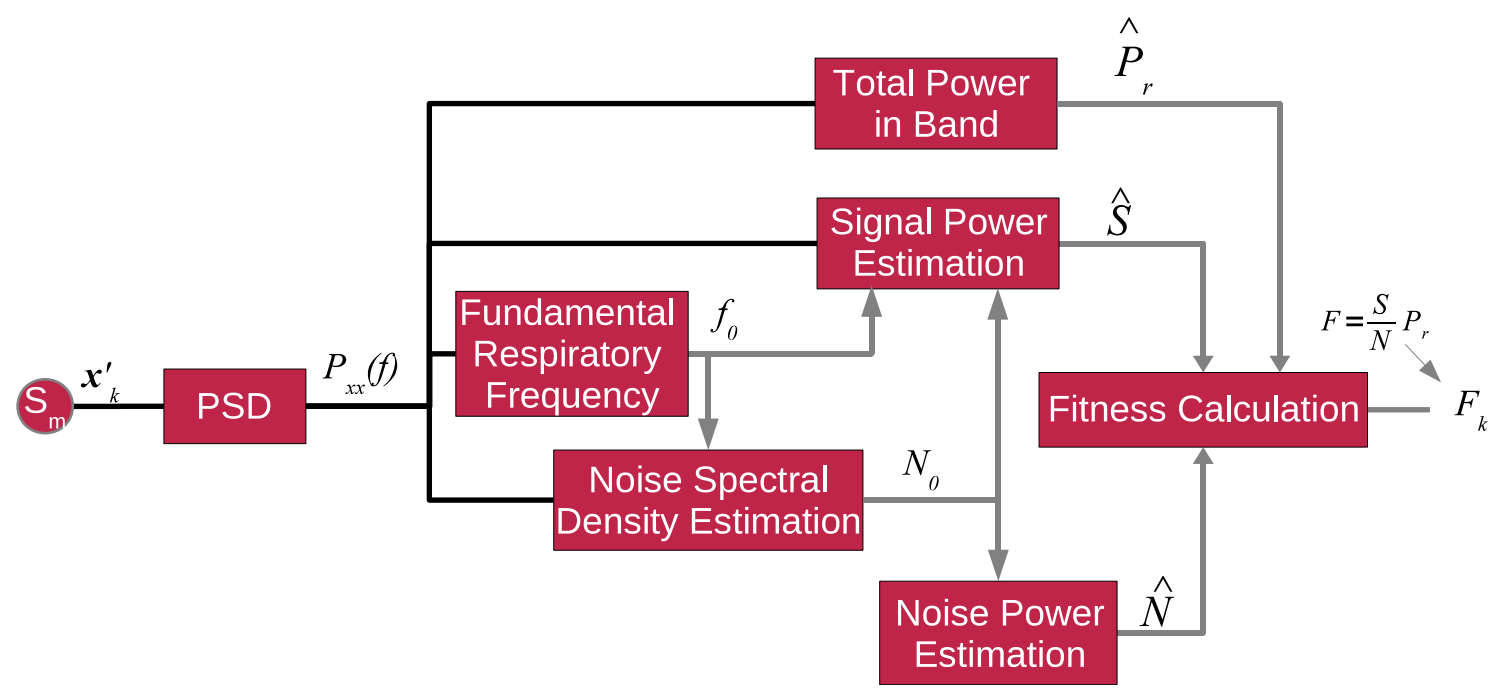

Figure 6.2: Block diagram of spectral ratio fitness calculator for selection combining

segment are small. However, for highly varying signal periods, there may be multiple signal lobes and spectral parameters may not be well estimated since part of the signal power will be assigned as noise. Nonetheless, for comparison between sensors of the same input signal, the same poor assignment occurs at each sensor and relative parameter values may still hold. A final caveat is that at low signal powers, interference can masquerade as a signal peak and lead to parameter estimation error.

\subsubsection{Methods of Selection Combining}

Current selection combining algorithms use a single feature of the breathing signal to characterize the fitness. Here, an approach will be described that makes use of two spectral features, making it less sensitive to both movement and breathing irregularity. The proposed spectral ratio selection method takes advantage of the periodicity of the breathing signal to discern the breathing signal from movement. Fig. 6.2 shows the block diagram of the fitness calculator for this method.

Signal power, $S$, and noise power, $N$, and total in band power $P_{r}$ are estimated. as described previously in Sec. 6.2.1. Recall that $P_{r}$ has been used previously in 
selection combining by Niizeki et al. [73] and Nishida et al. [15] as it own fitness measure. Here, this measure is combined with the SNR to form a spectral ratio.

The spectral ratio fitness at the $k$ th sensor, $F_{k}$, is calculated as:

$$
F_{k}=\frac{\hat{S}_{k}}{\hat{N}_{k}} \hat{P}_{r_{k}}
$$

Strongly rhythmic signals with strong powers stand out compared to signals with

noise and movement corruption in the $\frac{S}{N}$ term. When breathing is not rhythmic, the $P_{r}$ component allows the method to fall back to the spectral power method. This method is labeled "Sel. (SNR-PSD)" in the rest of this chapter, while the method using only $P_{r}$ is labeled "Sel. (PSD)".

In addition "Sel. (SNR-PSD)" and "Sel. (PSD)", a third method using only SNR is also proposed for the sake of comparison. This method is the same as the spectral ratio method, without the $\hat{P}_{r}$ term and is labeled "Sel. (SNR)."

\subsubsection{Methods of Weighted Summation}

Maximal ratio combining (MRC) [114], [115] is a type of signal combining that has not yet been applied to breathing signal fusion and is proposed here to increase the SNR of the output breathing signal. MRC is designated as "maximal" since the output SNR of the signal is maximized in traditional communication diversity systems with deterministic signals and Raleigh fading environments. MRC summation weighting is given in (6.3):

$$
g_{k}=\frac{\sqrt{S_{k}}}{N_{k}}
$$

where $S$ is signal power and $N$ is noise power. It can be shown that this maximal SNR is the sum of all the input SNR in the traditional system [114]. Let $\gamma_{k}$ denote 


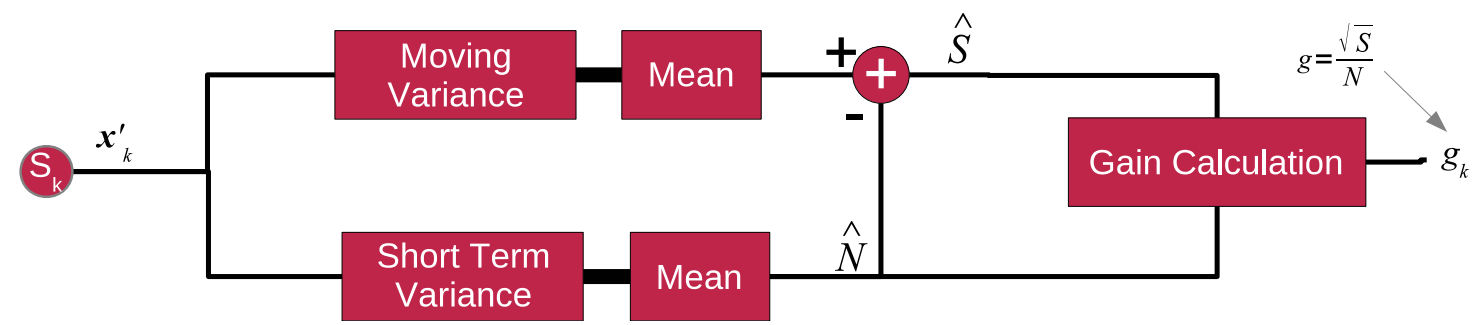

Figure 6.3: Block diagram of method to calculate MRC gain from variance

the SNR of sensor $k$. Then the output $\gamma \mathrm{SNR}$ is

$$
\gamma=\sum_{k=1}^{K} \gamma_{k}
$$

If all $K$ sensors have the same average SNR, $\gamma_{i n}$, then an $K$ sensor system increases SNR by a factor of $K$ over a single sensor system [114], [170]:

$$
\gamma=K \gamma_{i n}
$$

In ambient environments, with non-deterministic signals, the estimation of signal power and noise power is more difficult than in the traditional application of MRC in communication signal processing. Methods to estimate these parameters are required before MRC can be applied. Three methods of calculating the gains are detailed in the next sections. The first uses the temporal signal to estimate the signal and noise power from its variance while the second uses spectral power estimates. The third is an extension of the second and uses an estimate of the signal provided by an EGC combiner to first estimate the respiratory frequency before calculating MRC gains.

Power in a zero-mean signal can be calculated from its variance. Fig. 6.3 proposes a method for estimating signal power and noise power this way. If we assume that short term signal fluctuations are due to noise, but longer term fluctuations come from the respiratory motion, then noise power can be calculated by short term variance 
and signal power from longer term variance.

A short window of length $W_{S}$, is applied to capture noise fluctuations. A length of one second was selected as it usually represents less than a quarter cycle during regular breathing and even when breathing extremely quickly (up to 60 BPM), it represents less than a full breathing cycle. The samples inside the window are detrended to remove the longer fluctuation from the respiratory signal and the variance, $v_{N 1}$, is calculated on the detrended signal. This process is repeated by sliding the window along, sample by sample, until $E-W_{S}$ variances have been calculated, where $E$ is the epoch or segment length over which the gains are being calculated. The mean of these variances is the estimated noise power.

A longer window, of length $W_{L}$, is applied to capture the respiratory action. Fifteen seconds is chosen as this window size, to contain at least a single breath if the subject is breathing very slowly (4 BPM), or multiple breaths at higher breathing rates. Again, the windowed signals are detrended and variances, $v_{S i}$, calculated before sliding the window over by a sample and repeating the process $E-W_{L}$ times. A total power estimate $P_{t}$ is the mean of the calculated variances, so the signal power estimate is

$$
\begin{aligned}
S & =P_{t}-N \\
& =\left(\frac{1}{E-W_{L}} \sum_{i=1}^{E-W_{L}} v_{S i}\right)-\left(\frac{1}{E-W_{S}} \sum_{i=1}^{E-W_{L}} v_{N i}\right)
\end{aligned}
$$

Note this algorithm has been improved from the one published in [54], as it takes into account the noise power present in the respiratory window.

Signal and noise power can also be calculated from the power spectral density (PSD), as detailed in Sec. 6.2.1. Fig. 6.4 is a block diagram of a system to calculate MRC gains using the PSD. This method is labeled MRC (PSD). 


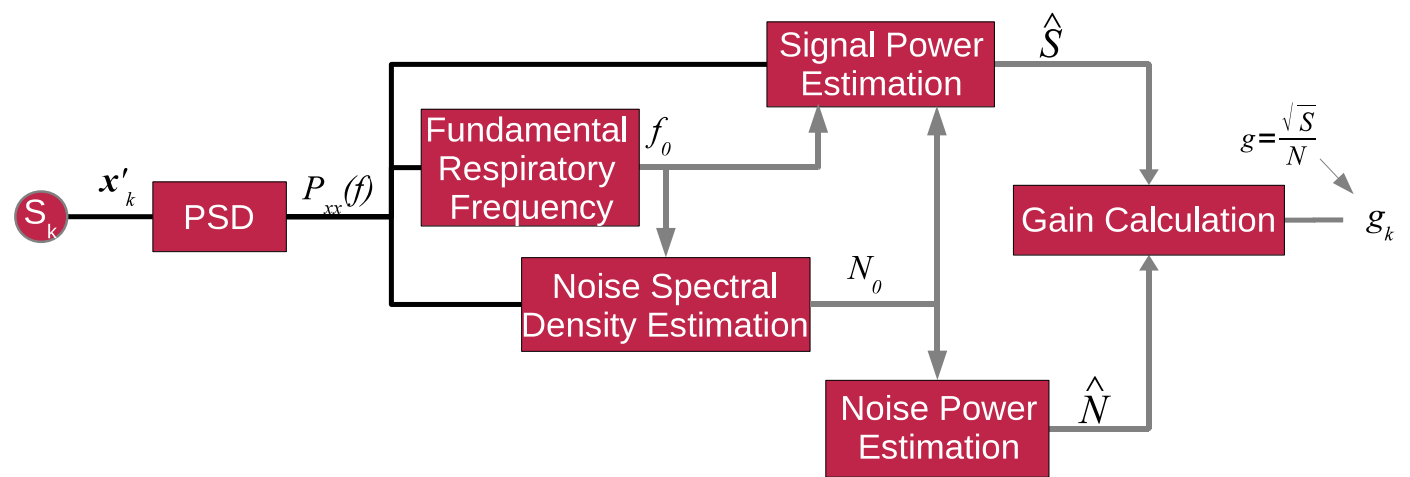

Figure 6.4: Block diagram of method to calculate MRC gain from power spectral density

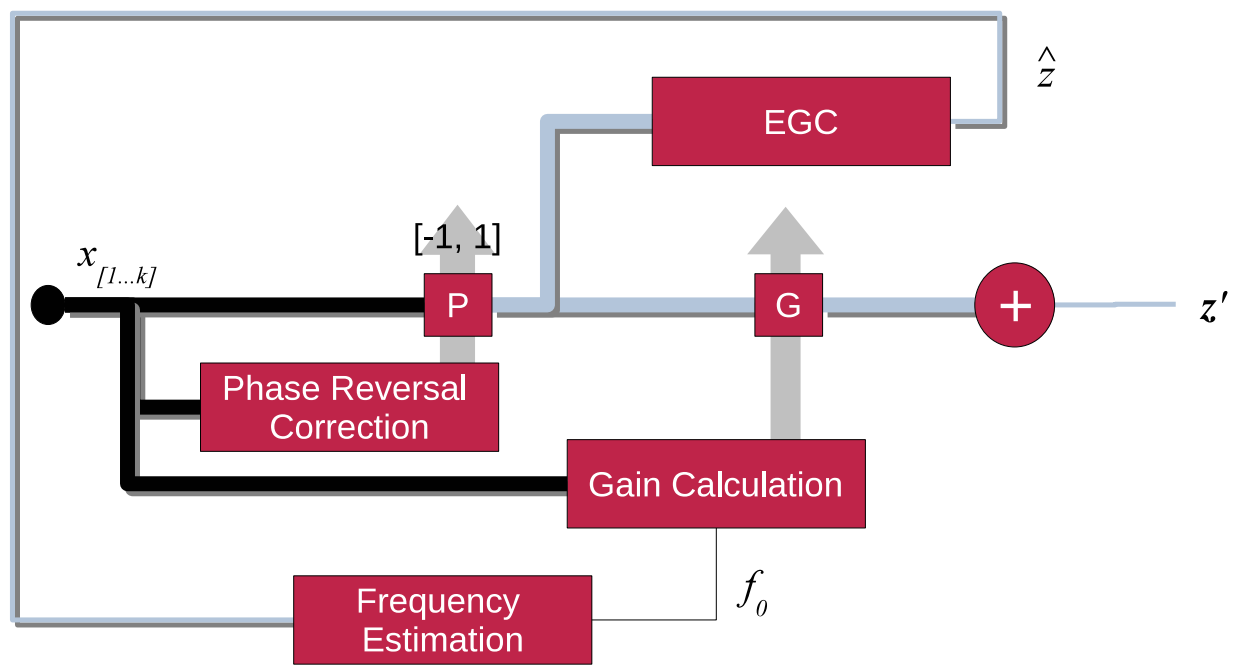

Figure 6.5: Block diagram of MRC (PSD+EGC) method. EGC is used to estimate the respiratory frequency.

The MRC method presented in the previous section treated each sensor independently; fundamental frequencies were calculated for each sensor individually. For sensors with weak or non-existent breathing signals, the chosen fundamental frequency may belong to interference rather than breathing. Erroneous signal and noise estimates ensue.

Here, the MRC method is extended by producing a frequency estimate first using an initial signal estimate by EGC. Fig. 6.5 presents this method, labeled MRC $(\mathrm{PSD}+\mathrm{EGC})$. The initial signal estimate is obtained using EGC, a simple but effec- 
tive combining method that does not require complex operations. MRC gains at each sensor can then be calculated using the estimated fundamental frequency, rather than each sensor calculating their own.

\subsubsection{Methods of Blind Source Separation}

Blind source separation allows separation of signal sources without knowing about the signals. Three methods of blind source separation are proposed. The first uses PCA, the second combines principal component analysis and independent component analysis, and the third applies the adaptive trend analysis from the previous chapter as a form of principal components analysis.

\section{Principal Component Analysis}

PCA for breathing signal fusion was reviewed in Sec. 2.2.4. Previous work extracted respiratory motion from just the first component, but required movement detection and iteration when movements were detected [43]. Assuming however that the breathing signal is simply part of another component allows this to be simplified, although it does require that a signal is selected from among the first few components each time. This method is applied here, using the selection combining methods of Sel. (SNRPSD) outlined in Sec. 6.2.2 to select the best candidate component since preliminary testing showed it to be most resilient of the three tested selection methods. This is effectively a hybrid system of PCA and selection combining and is labeled "PCA (Sel.)" and depicted in Fig. 6.6. Selection of the final output was made between the first three PCA components. 


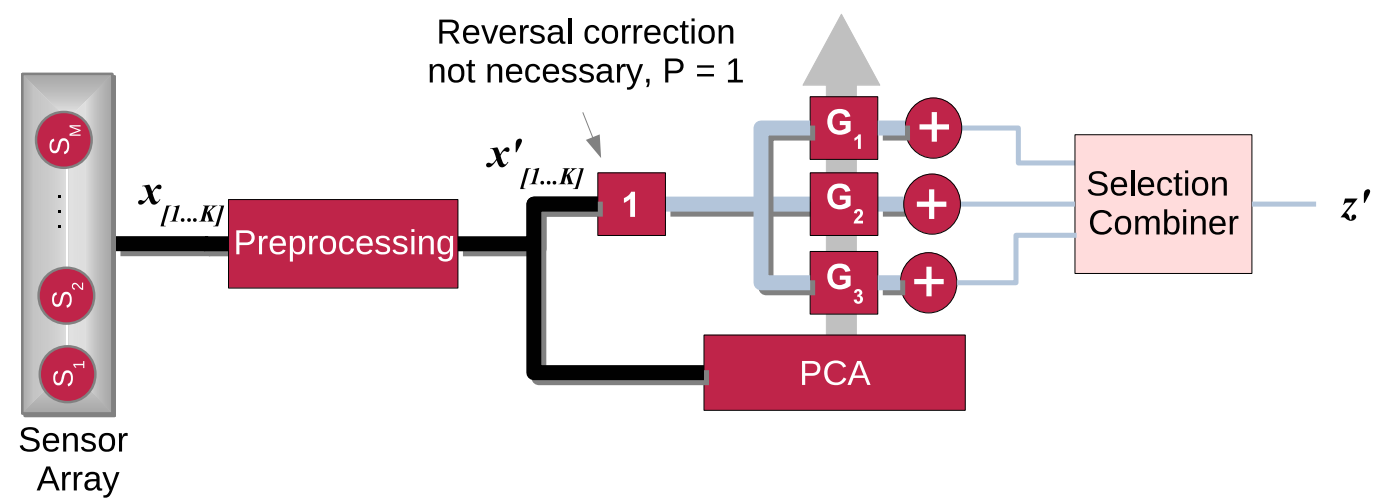

Figure 6.6: Block diagram of PCA with component selection

\section{Independent Component Analysis}

Here we build on the work of Uchida et al. [67], who applied independent component analysis (ICA) from just two sensors, to examine ICA from many sensors. For an array that includes more than three sensors, the ICA problem becomes overdetermined. That is, the number of sensors $K$ is larger than the number of sources, $N_{\mathrm{SRC}}$. To reduce dimensionality, PCA is often applied first [119], [171]. For $N_{\mathrm{SRC}}$ sources, the first $N_{\text {SRC }}$ principal components are collected. With the number of dimensions reduced to the number of expected sources, ICA is applied. This method is labeled "ICA-PCA" and shown in Fig. 6.7. As in PCA, selecting the appropriate output is required and was done here in the same manner as PCA Sel. ICA with PCA dimension reduction can be implemented using Hyvarinen's "FASTICA" algorithm, which provides a practical optimization of ICA components separated by minimizing mutual information [119]. These algorithms are available as a package for MATLAB.

\section{Decision Directed Trend Analysis}

The method presented in the preceding chapter (Sec. 5.3.4) for adaptively determining the polarities for reversal detection can be used for combining as well, and can be 


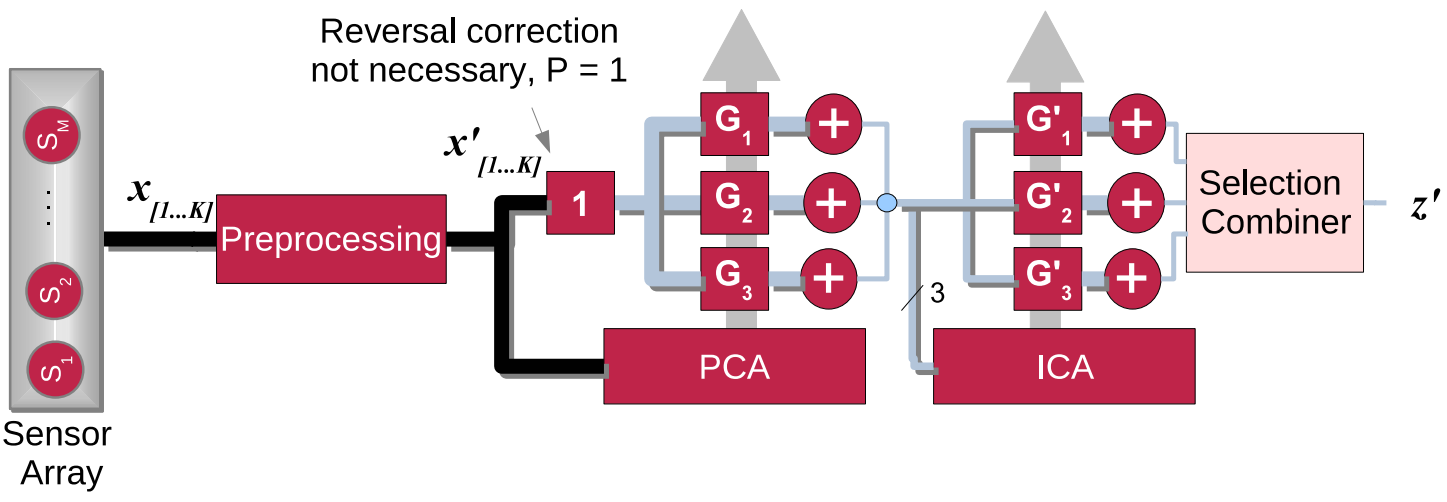

Figure 6.7: Block diagram of ICA using PCA for dimension reduction and selection combining to select the final signal

viewed as analogous to adaptive PCA, but making use of correlation rather than covariance. The output of that adaptive process provided a set of weights whose sign indicated phase reversal. The weights themselves also give an indication of how correlated each of the signals are to the general trends in the data. By using the weights as gains, not only can phase reversals be corrected, but signals with more correlation to the general trends in the data are emphasized. While this is an adaptive process, constant adaption of fusion weights would affect the breathing signal amplitude. For applications that analyze signal amplitude, weights should be updated and applied on a segment-wide basis, rather than sample by sample. To apply it on a segment, adaptation is run over the first $L$ samples and the weights at the end of this adaptation are used. Alternatively, for offline processing, any $L$ samples within the segment can be used.

\subsection{Experiment \#1: Signal-Level Fusion in Ad- verse Conditions}

The ambient environment and quasiperiodic breathing signals were hypothesized to play a role in the performance of fusion methods. In this experiment, the effects 
of interference, signal availability, and non-stationary behaviour were evaluated by comparison between the proposed and conventional methods of signal-level fusion.

Three types of interference were studied, namely white noise, drift, and semicorrelated burst interference from movement artifact. There effect of reduced signal availability was also explored. To probe these behaviours, the test sets created in Chapter 3 were put to work. The corrupted sets of the "Reduced Availability Set", the "Noise Set", and the "Drift Set", as well as the intrinsically disordered sets of the "Movement Set", and the "Breathing Set" were used.

Each of the epochs in the test sets were input to each of the fusion algorithms after phase alignment using DDSRD of the previous chapter. The resulting output quality was assessed by comparison to the respibands through the correlation coefficient $r$. Movement was ignored during evaluation but no mitigation of movement was performed in the input signals.

For the movement set, movement epochs were binned by length of movement. Length of movement can be related to movement intensity. Not only do longer movements at equal powers produce more movement energy in an epoch, but longer movements actually usually correspond to stronger movement power too. The bins were: short $<1 \mathrm{~s} ; 1 \mathrm{~s}<=$ mid-short $<2.5 \mathrm{~s} ; 2.5 \mathrm{~s}<=$ mid-long $<5 \mathrm{~s} ; 5 \mathrm{~s}<=$ long. Breathing irregularity was also binned for the breathing set, this time using the standard deviation of the breathing intervals. The higher the variability in these intervals, the more disorder and non-stationary behaviour appears in the data.

In addition to the selection To examine the selection accuracy of each algorithm, the selected sensors were compared to the optimal sensor selection, the sensor with the best correlation to the respibands. 


\subsubsection{Results}

For each evaluated test set, four sets of plots are shown. The first three show the methods grouped in fusion categories of selection, diversity combining, and blind source separation, respectively. The last displays the best new methods for comparison across categories.

\section{Interference}

Interference in the form of noise, drift, and movement were studied. As the SNR was increased, Fig. 6.8 shows the effect on the correlation to respibands. All methods show reduced signal quality as noise is added, but in particular, the advantage over EGC enjoyed by the MRC (PSD) and MRC (Var) methods is narrowed until no advantage is perceived as noise is added. As noise is added, the MRC (PSD+EGC) method maintains its advantage over EGC. In fact, the gap widens slightly at high levels of noise. Sensor selection is the most disadvantaged when noise is present, and continues to drop as noise is added.

As drift in the sensor signals is increased, Fig. 6.9 shows the effect on the correlation to respibands.

As movement energy increases in the epochs, Fig. 6.10 shows that all correlations to the respiband decrease. Although optimal selection resulted in stable signal quality, the selection methods, and in particular the Sel. (SNR) method, deteriorated. The Sel. (SNR-PSD) method in this instance is better than the sum of its parts, faring better than both the Sel. (PSD) and the Sel. (SNR) method.

From the diversity methods, EGC was the best performer. This method blindly sums all of the signals and the movement does not affect its choice of gain. It does still lose some ground, perhaps one or two of the sensors are affected by movement after the movement has been declared finished. The MRC (PSD+EGC) method is 

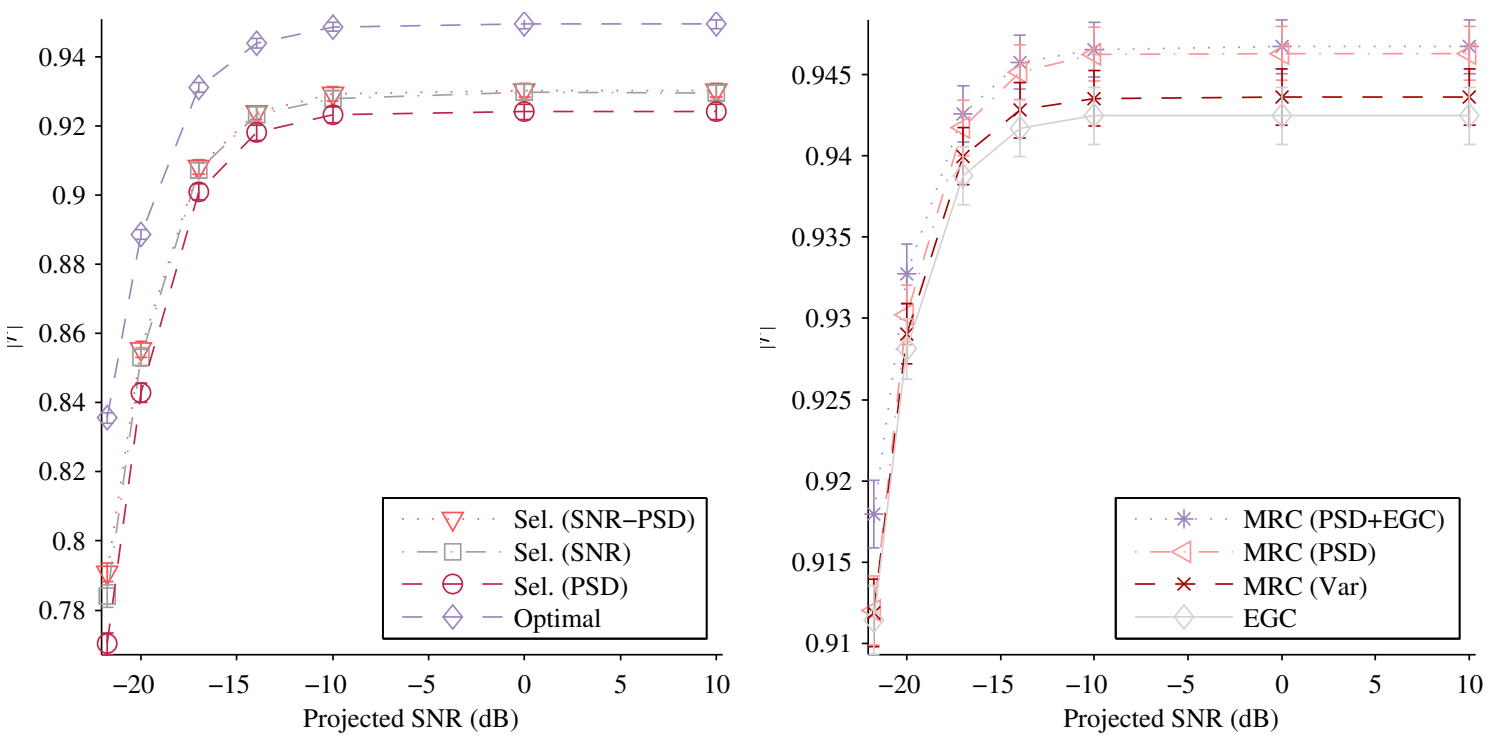

(a) Selection methods in noise

(b) Linear diversity methods with noise: MRC (PSD) and MRC (Var) methods have degraded performance in noise, but MRC(PSD+EGC) improves upon their performance

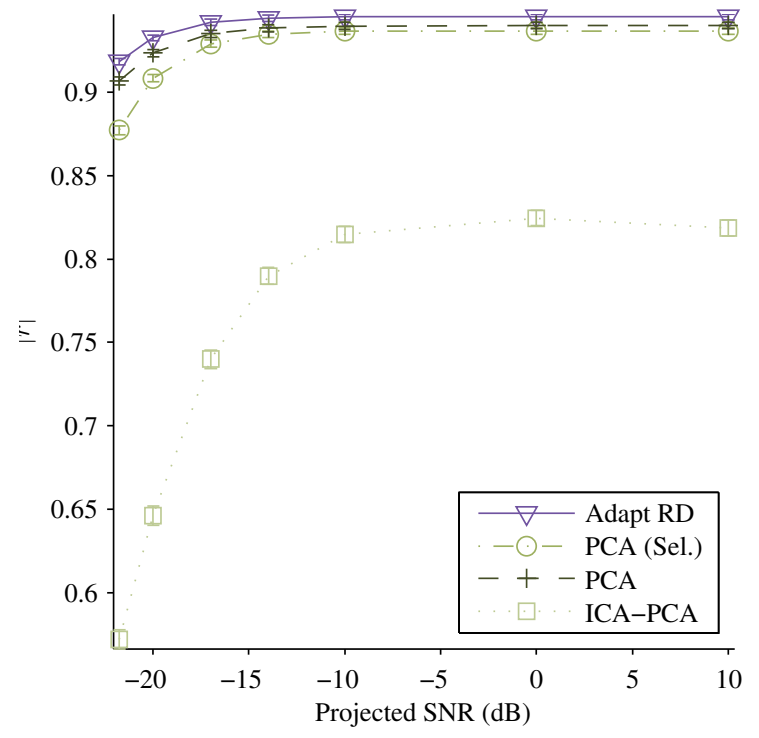

(c) Blind source separation in noise

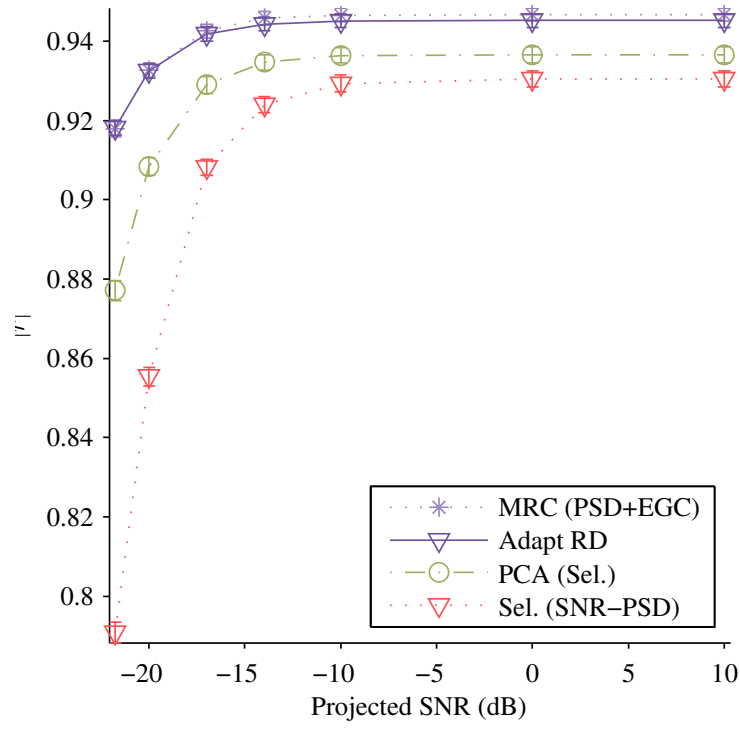

(d) Comparison of all new methods in noise

Figure 6.8: Mean correlation coefficient of method outputs compared to respibands by percentage of added noise corruption 


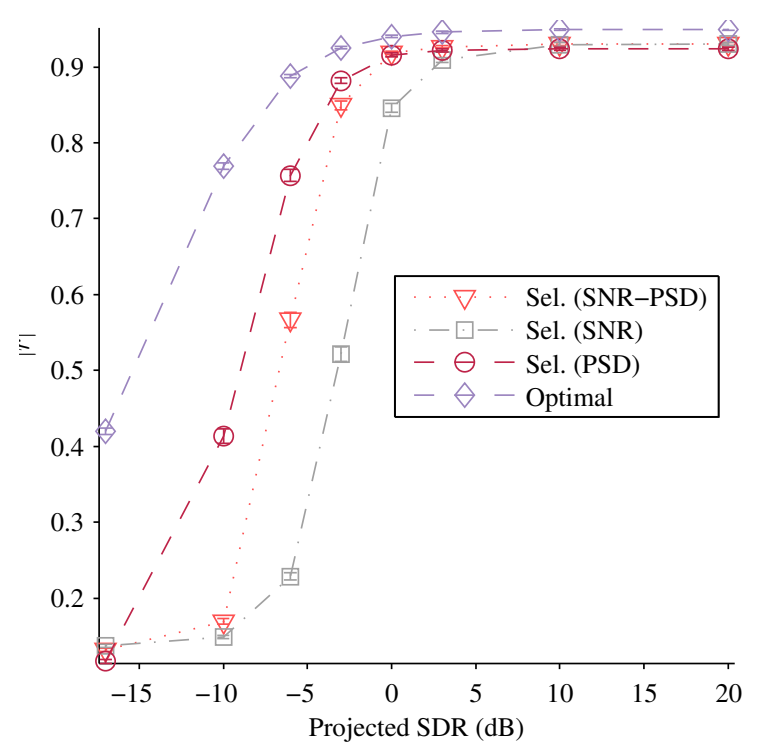

(a) Selection methods in drift

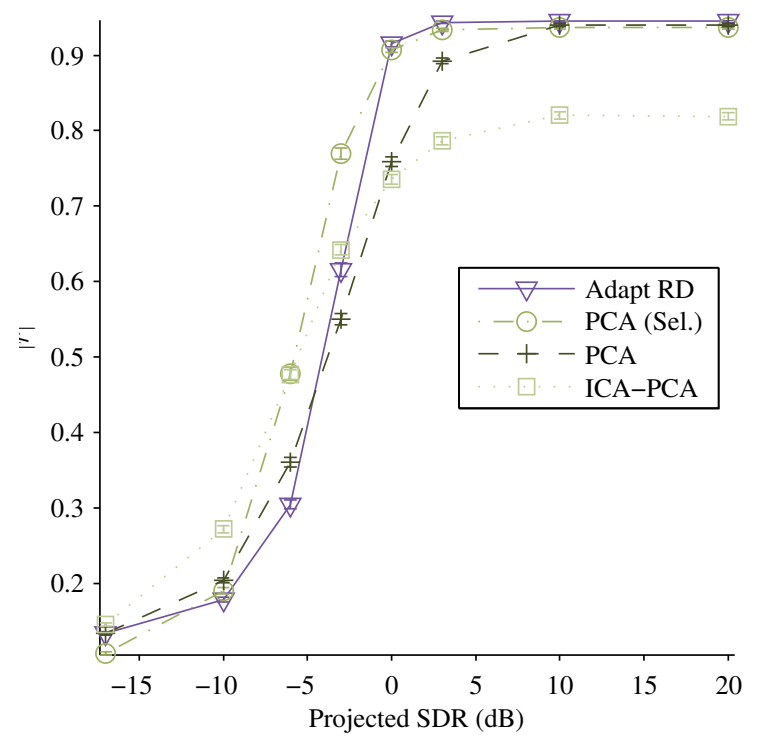

(c) Blind source separation in drift

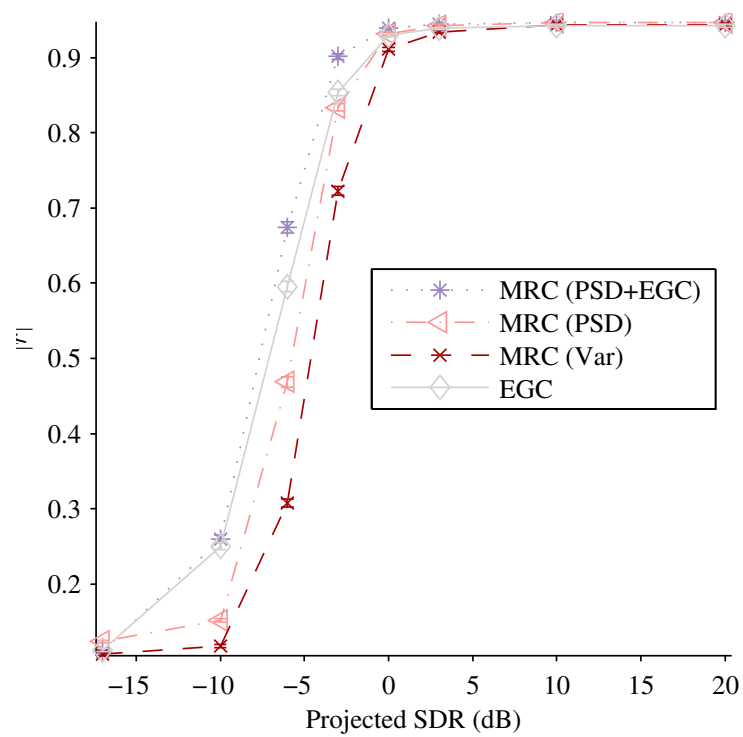

(b) Linear diversity methods with drift: MRC (PSD) and MRC (Var) methods have degraded performance in drift, but MRC(PSD+EGC) improves upon their performance

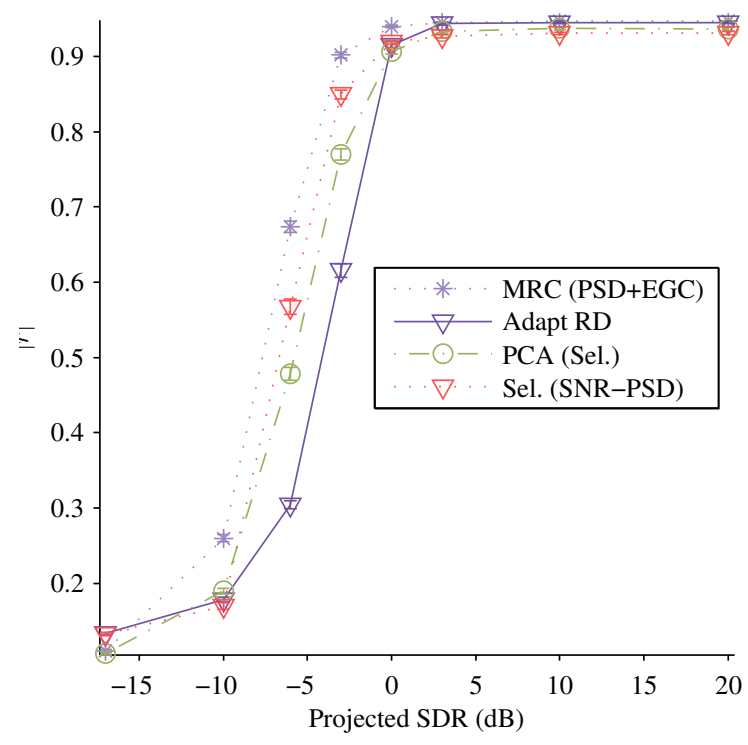

(d) Comparison of all methods in drift

Figure 6.9: Mean correlation coefficient of method outputs compared to respibands by percentage of added drift corruption 


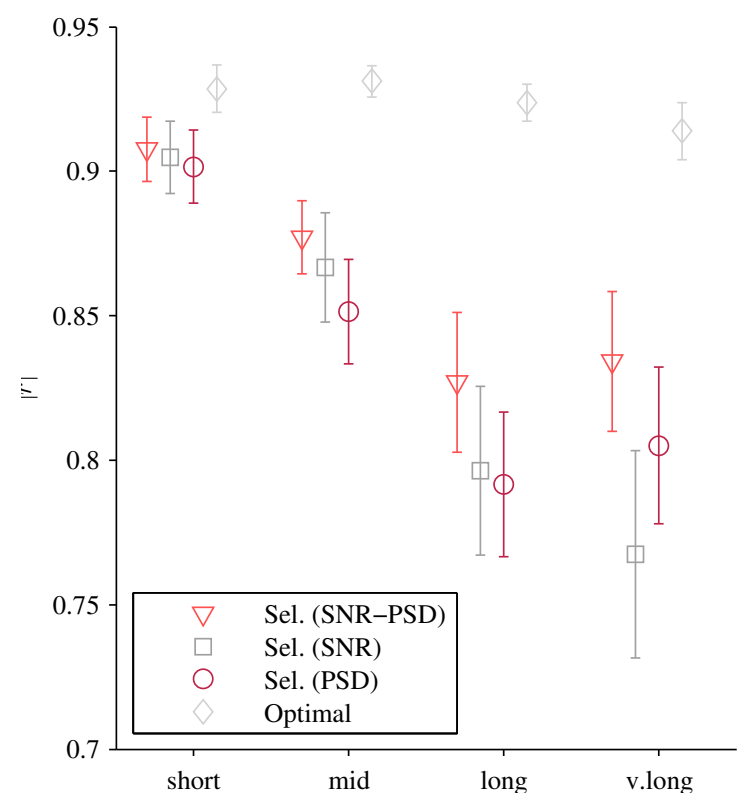

(a) Selection methods during movement

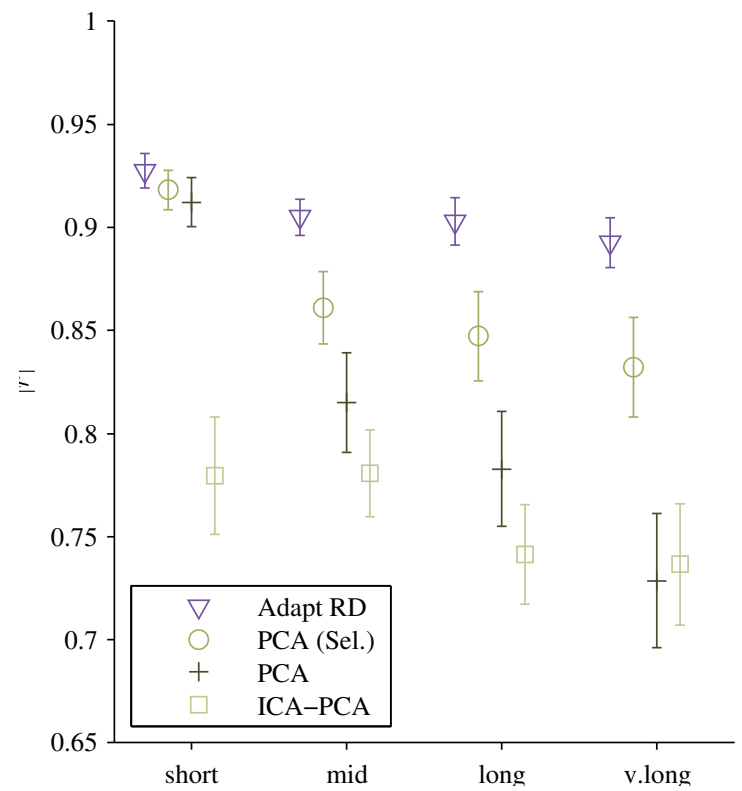

(c) Blind source separation during movement

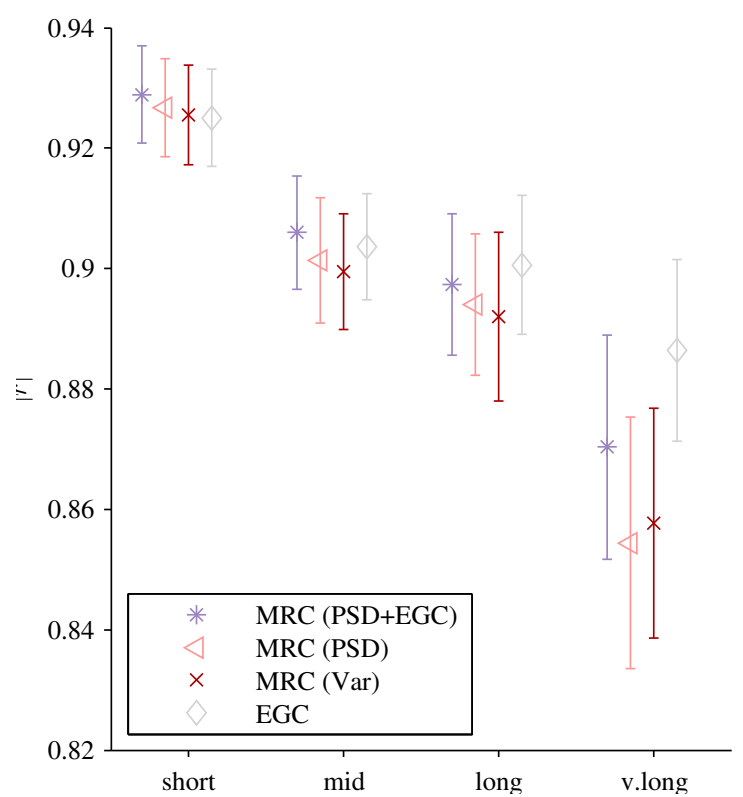

(b) Linear diversity methods during movement

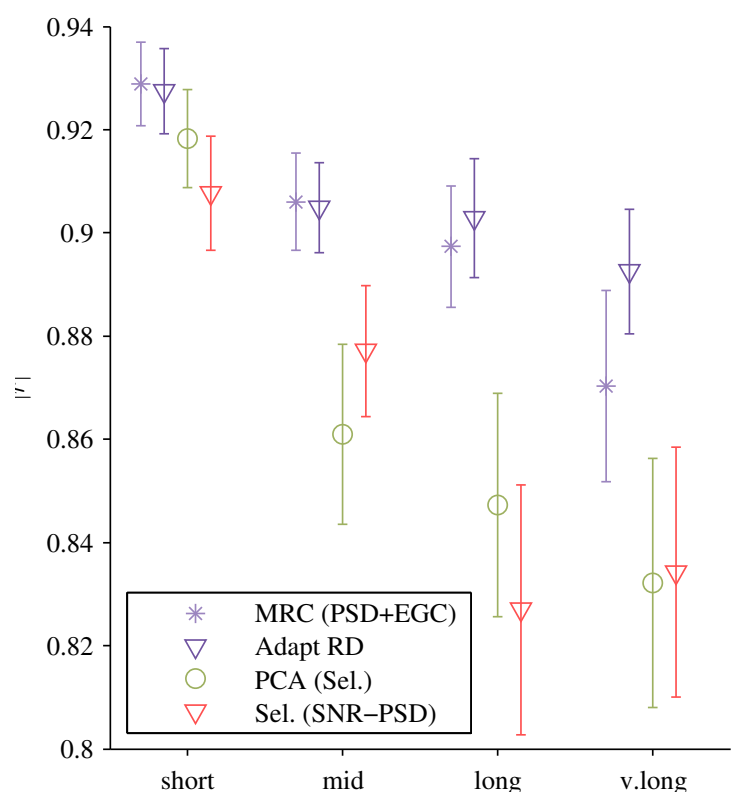

(d) Comparison of all new methods during movement

Figure 6.10: Mean correlation coefficient of method outputs compared to respibands by duration of movement (short $<1 \mathrm{~s} ; 1 \mathrm{~s}<=$ mid-short $<2.5 \mathrm{~s} ; 2.5 \mathrm{~s}<=$ mid-long $<5 \mathrm{~s} ; 5 \mathrm{~s}<=$ long) 
able to put its EGC input to good use and overcome some of the spectral problems that hinder the MRC (PSD) method during movement.

The blind source separation methods were the least affected by movement. The advantage of selecting the PCA component is seen here, where PCA drops while PCA (Sel.) is able to hold some of its ground. The Adapt RD method is very resistant to the movement and only drops a small amount.

\section{Availability}

As the number of sensors with a good breathing signal is varied, Fig. 6.11 shows the effect on the correlation to respibands. As in theoretical expectation for the SNR, the selection method levels off and increases at a lower marginal rate for every added sensor than EGC. MRC provides better results than EGC. However, the method of obtaining the signal and noise power estimate makes a difference, as SNR climbs at a slightly higher rate for MRC (PSD) and MRC (PSD+EGC) than for MRC (Var).

\section{Variability in Breathing Period}

Fig. 6.12 displays how variability in the breathing period affects the fusion methods. Generally, all methods are equally affected. However, the spectral-based selection methods are slightly more affected than the others and drop off more quickly than the Adapt RD and MRC (PSD+EGC). The SNR method is particularly affected by breathing irregularity and just a few added samples of irregularity reduced its advantage over the Sel. (PSD) method. The PCA (Sel.) method is also affected since it depends on selection. In this instance, without interference, PCA without selection performs better. It is somewhat surprising that the MRC (PSD+EGC) method does not drop off too, given its reliance on spectral estimations. 

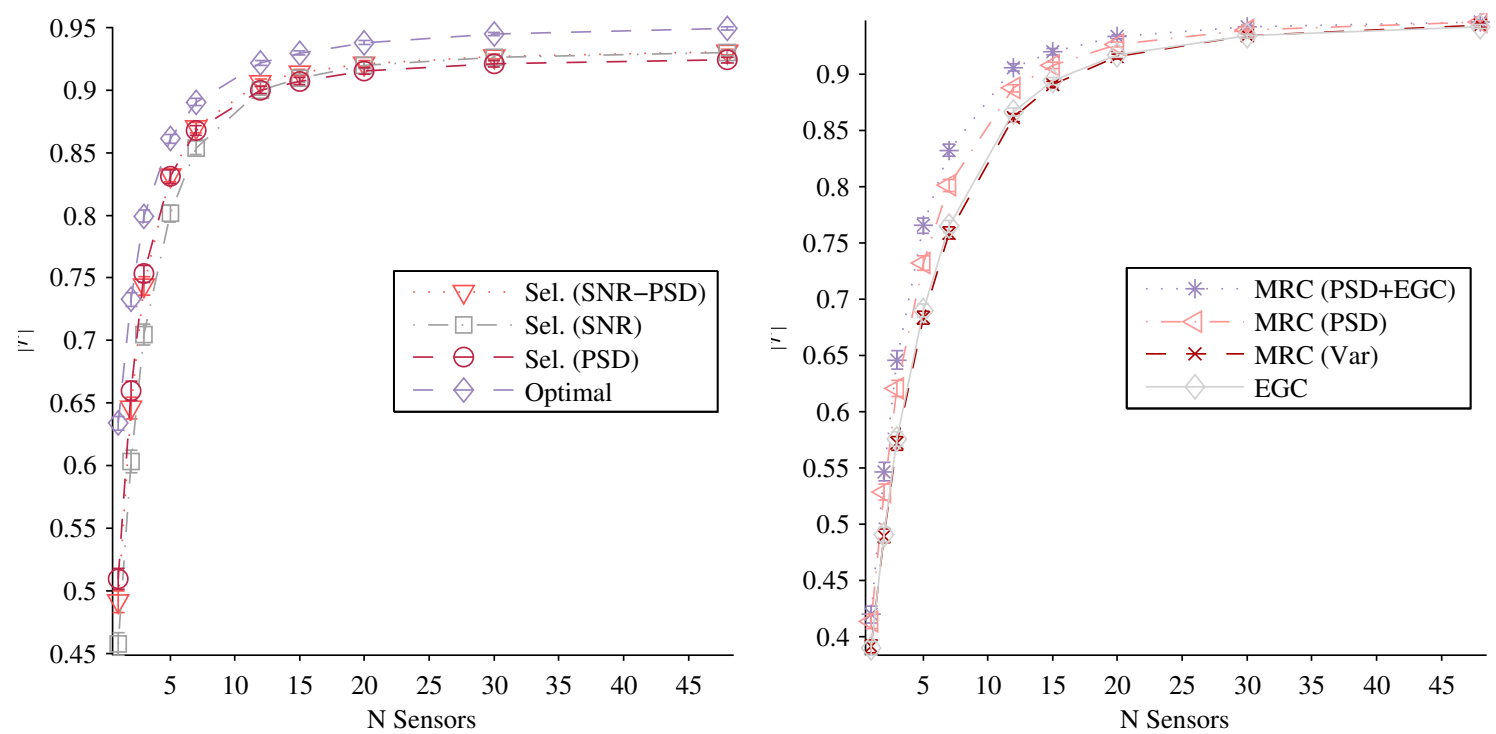

(a) Selection methods for reduced availability

(b) Linear diversity methods with reduced availability: MRC (PSD) and MRC (Var) methods have degraded performance with reduced availability, but MRC(PSD+EGC) improves upon their performance
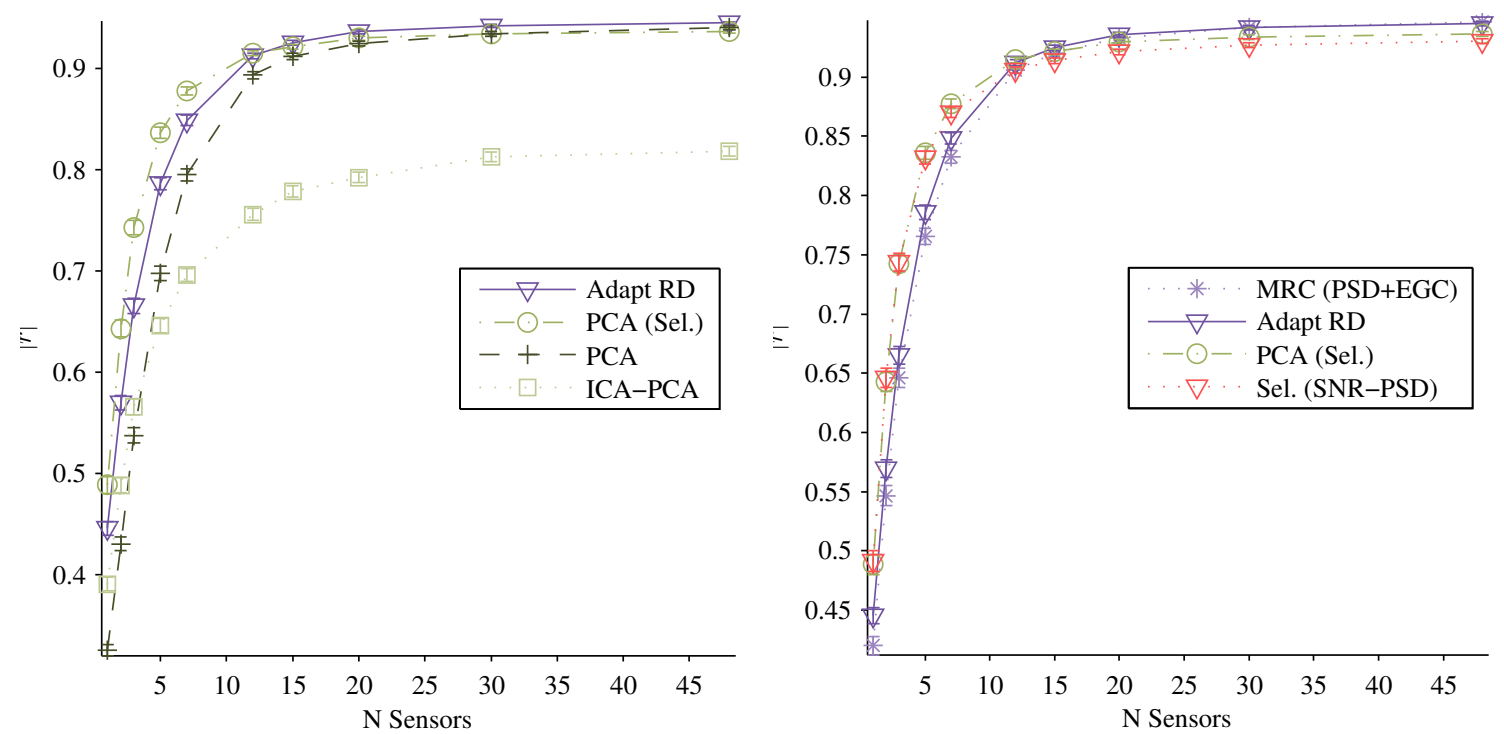

(c) Blind source separation with reduced avail-(d) Comparison of all methods for reduced availability ability

Figure 6.11: Mean correlation coefficient of method outputs compared to respibands by number of sensors with valid breathing signals 


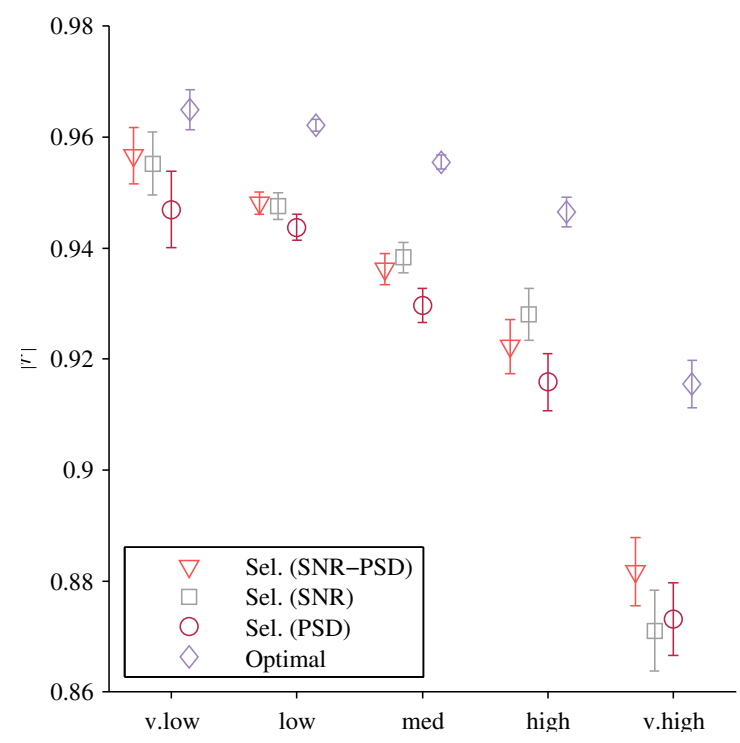

(a) Selection methods

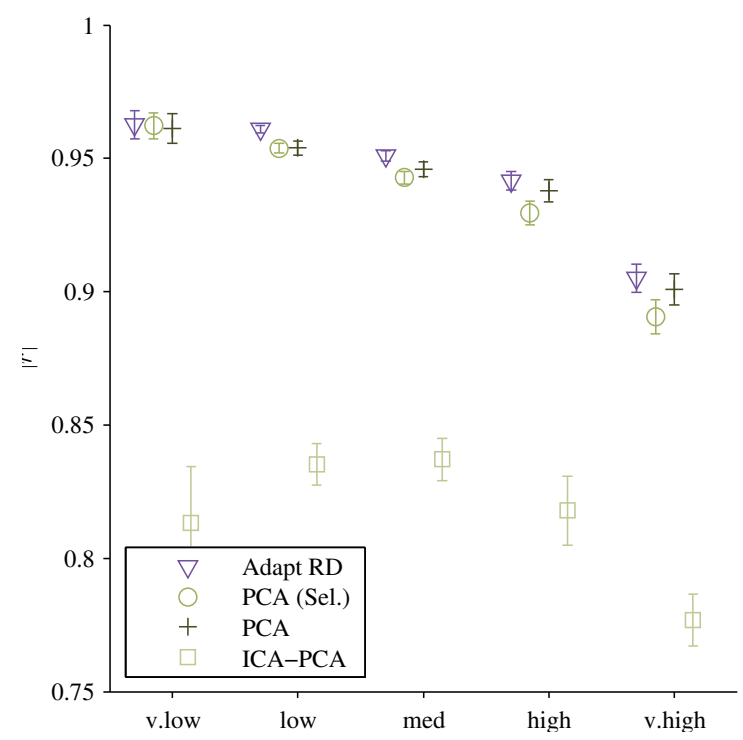

(c) Blind source separation methods

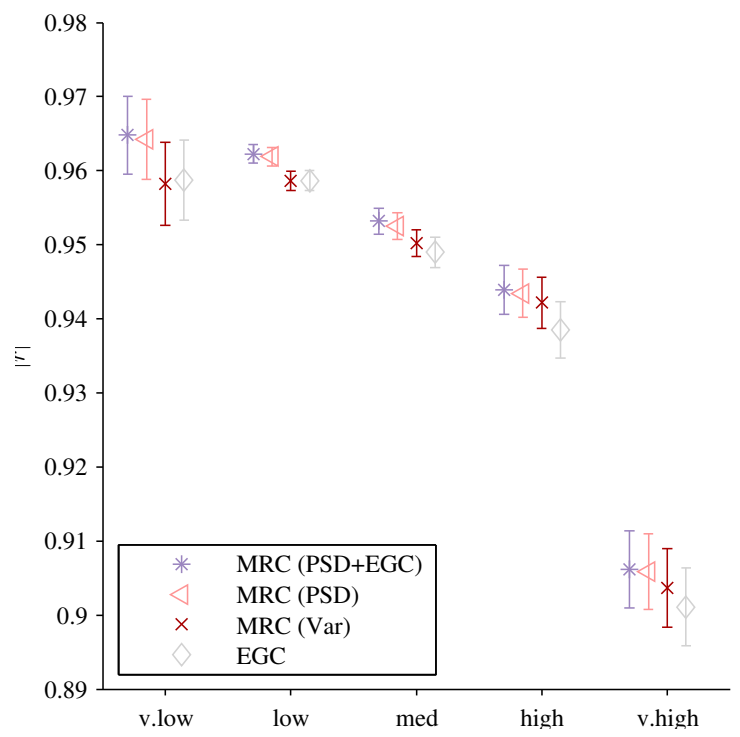

(b) Linear diversity methods

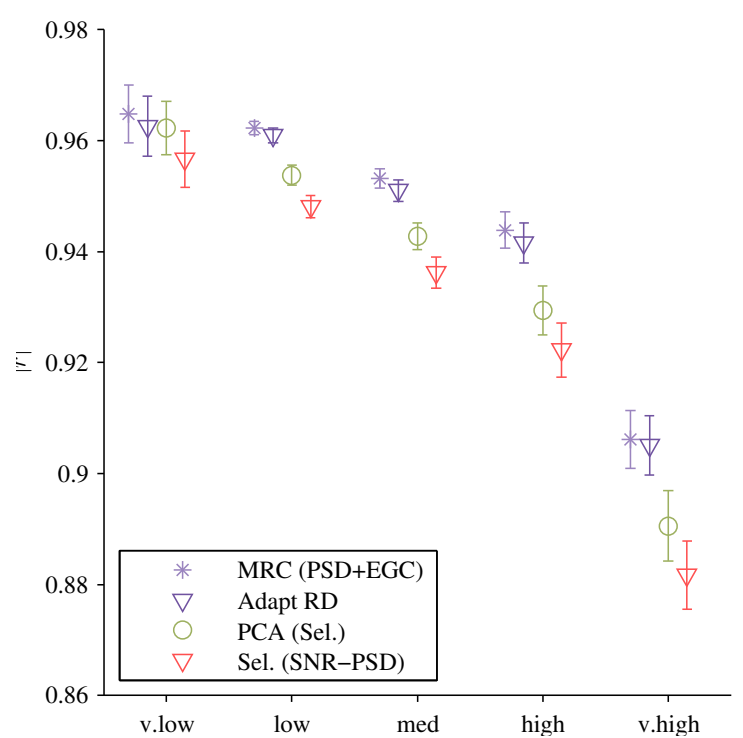

(d) Comparison of all methods

Figure 6.12: Mean correlation coefficient of method outputs compared to respibands by amount of period variability, measured by the standard deviation of the breathing intervals of the respibands (v.low $<1.5 \mathrm{~s} ; 1.5 \mathrm{~s}<=$ low $<3 \mathrm{~s} ; 3 \mathrm{~s}<=$ med $<4.5 \mathrm{~s}$; $4.5 \mathrm{~s}<=$ high $<6 \mathrm{~s}$; v.high $>=6 \mathrm{~s}$ ) 


\subsubsection{Discussion}

The MRC (PSD+EGC) and Adapt RD methods provided better estimates of the breathing signal overall. For patients who are constantly moving, such as those with restless leg syndrome, and for those with breathing disorder, these two methods are especially appropriate. However, if environmental conditions are poor, it is sometimes beneficial to choose another method. Both methods are not as good when signal availability is low, and Adapt RD can be affected by drift.

In stationary conditions, MRC yields the optimal weights to maximize SNR. In communications systems, where the signal and noise powers of harmonic signals at known frequencies are being measured, this optimality generally holds. However, in this application, determining the signal and noise power is not as clear-cut. Spectral SNR estimates for non-harmonic, quasi-periodic signals with multiple sources of interference are only at best relative measurements. Spectral estimates may not accurately assess what is "signal" and what is "noise". The MRC methods presented here adopt a model of a respiratory signal that is mostly sinusoidal with negligible signal power in the harmonics. Spectral signal estimates included only the power in the fundamental lobe and ignored the power contained in harmonics, which could contain appreciable power. It is also possible to get false estimates due to periodic, undetected movements, or the systemic variance in the periodogram. Similarly, irregular breathing rates that smear the signal power across many frequencies may also result in a poor signal power estimates.

In as much as the spectral signal and noise estimates are not necessarily accurate, they tended to improve results compared to temporal measures. This was seen generally by comparing MRC (PSD) and the MRC (Var) method. MRC (PSD) did have poorer results when movement artifact was present, which was likely due to a fallacious peaks appearing in the PSD. Selection of such a peak as a fundamental 
frequency leads to poorly assigned signal gains. By using the frequency estimate from EGC, MRC (PSD+EGC) negated MRC (Var)'s advantage during movement. Movement may violate the assumption of uncorrelated and stationary noise that is required in $\mathrm{MRC}$, but this was not shown to be particularly a problem for MRC $(\mathrm{PSD}+\mathrm{EGC})$.

Generally, the selection methods perform poorly compared to the methods that make use of more sensors. However, there are a few scenarios where this method may be preferred. If the bed occupant is small and is expected to cover few of the sensors, sensor selection is practical. Conversely, if the bed occupant is obese and many of the sensors will saturate, leaving few with an available respiratory signal, sensor selection is also useful. Finally, if simplicity is the main objective, the selection method does not require reversal detection and is simpler than PCA (Sel.).

Of the selection methods, the spectral ratio method provided a good trade-off for performance when tested in conditions of both breathing irregularity and movement artifact. For all selection methods, there is still room for improvement compared to the correlations achieved by choosing the optimal sensors, particularly during strong movement. However, since the optimal selection method is retrospective, it has the advantage of hindsight when selecting the breathing signal. It is unlikely that any real-world algorithm would would do as well. Nevertheless, in the presence of limb movements, there may be some merit to its performance since there could be sensors unaffected by localized movements. Future work at improving sensor selection could yield big improvements.

The ICA-PCA method performed poorest of all of the methods. This method expects the signals to contain a linear mixture of the source signal, which is not the case. While the concept of a single respiratory effort signal is assumed, respiratory effort is made by a number of muscles in the body, principally the intercostals and 
the diaphragm. The single effort signal source does not actually exist. Additionally, sensor responses can be non-linear and non-uniform according to the location of loading with respect to the sensor, and vary from sensor to sensor, as explored in Chapter 4. Furthermore, the required three signal sources may not always be present in the signal. Indeed, the BCG may not have been often available and for clean records without appreciable interference, only a small noise signal would have been seen. Some tests were run with only two signal sources, but this did not measurably improve ICA-PCA performance. Chen et al. dismissed ICA as being unsuited to ambient breathing fusion because of the delays that were present in the signal between the sensors. Certainly delays are sometimes apparent between signals and contribute to the problem, although signal alignment is a more solvable problem [53] than the unknown non-linearity introduced in each signal.

The problem of differing breathing effort signals dependent on the sensor location may also affect the optimality of MRC combining, which requires that the multiple signals to be combined hold "closely similar copies [of] the desired signals [114]". However, the somewhat-similar copies were enough for MRC-PSD to produce one of the best results in most circumstances. The results of the signal availability test set highlight the advantage of having multiple sensors carry a somewhat-similar copy of the desired signal. All algorithms provided marked improvements as the number of sensors carrying the signal increased. When designing a bed-based sensor array system, spatial resolution is often chosen with position and posture classification in mind; however, it can also be advantageous to increase the number of sensors in an array to produce breathing signals of better quality. It is important to add more sensors by increasing the density of sensors beneath the torso rather than just adding to overall array dimensions. A larger number of sensors clustered beneath the torso would allow more sensors to carry breathing signals, but sensors added near the feet would add movement artifact without the benefit of supplemental breathing signals. 
In conditions with few sensors carrying the desired signal, an algorithm should allow for weightings of zero or very close to zero at sensors not carrying the signal. Otherwise, adding these signals into the output only adds noise. Although the MRC $(\mathrm{PSD}+\mathrm{EGC})$ does allow for very small weightings, the inherent variance of the power spectrum estimate means that some sensors will have an artificially high weighting.

Furthermore, since the breathing signal is not stationary, the power spectral method may underestimate the signal power, leading to artificially low weighting on the few sensors carrying the signal. Similarly, the selection method of Sel. (SNR) is adversely affected by these inaccuracies in SNR estimates.

\subsection{Experiment \#2: Validation of Experimental Results}

To see the impact of the fusion methods on regular analysis, the "Validation" set was analyzed. All of the 11 methods tested in the previous experiment were validated against the validation data set described in Sec. 3.3.4.

\subsubsection{Results}

The mean respiration rate error and the percentage of estimates within 1 BPM are shown in the barcharts of Fig. 6.13. This generally matched the signal quality results: methods with higher $|\bar{r}|$ estimated respiratory rate more accurately.

Table 6.1 provides a resume of results for the output signal quality of the validation set records.

Boxplots of all of the quality outputs were constructed for comparison between the methods, shown in Fig. 6.14. Student's right-tailed t-tests appraised the significance of the quality improvement of the top method, Adapt RD against all other methods, 


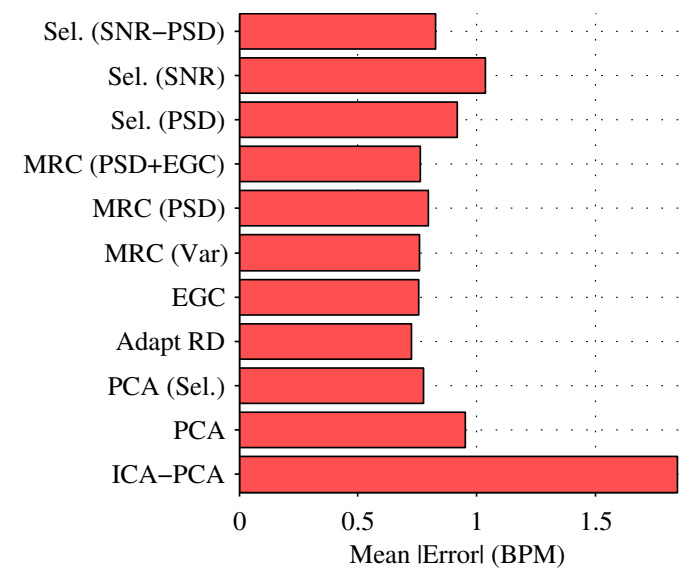

(a) Respiratory Rate Error (BPM)

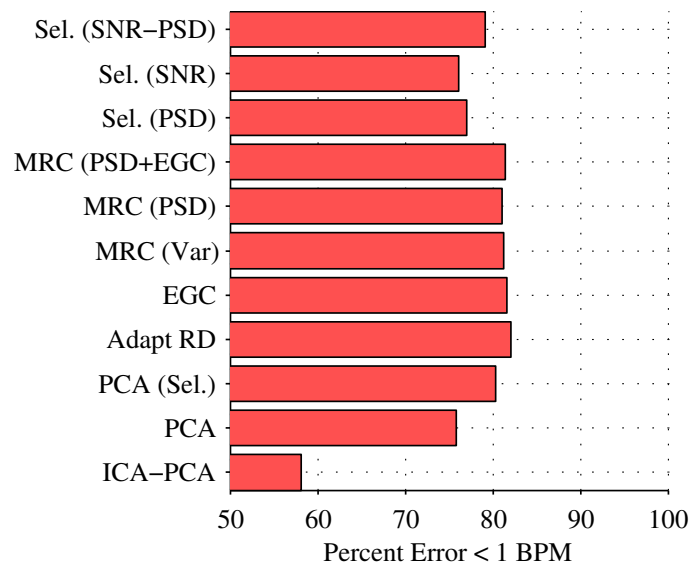

(b) Rate estimates within 1 BPM (\%)

Figure 6.13: Barcharts of respiratory rate agreement with respibands by fusion method

Table 6.1: Performance of breathing fusion methods applied to validation records

\begin{tabular}{|r||c|c|c|}
\hline & $|\bar{r}|$ & C.I.(low, high) & $r \geq 0.7(\mathbf{\%})$ \\
\hline \hline Sel. (SNR-PSD) & 0.864 & $(0.861,0.867)$ & 87.4 \\
\hline Sel. (SNR) & 0.846 & $(0.842,0.849)$ & 84.2 \\
\hline Sel. (PSD) & 0.848 & $(0.845,0.851)$ & 85.4 \\
\hline MRC (PSD+EGC) & 0.889 & $(0.886,0.892)$ & 90.4 \\
\hline MRC (PSD) & 0.887 & $(0.885,0.890)$ & 90.0 \\
\hline MRC (Var) & 0.884 & $(0.881,0.887)$ & 90.1 \\
\hline EGC & 0.887 & $(0.885,0.890)$ & 90.7 \\
\hline Adapt RD & 0.892 & $(0.889,0.894)$ & 90.7 \\
\hline PCA (Sel.) & 0.874 & $(0.871,0.877)$ & 88.1 \\
\hline PCA & 0.844 & $(0.840,0.847)$ & 82.6 \\
\hline ICA-PCA & 0.733 & $(0.729,0.737)$ & 64.3 \\
\hline
\end{tabular}




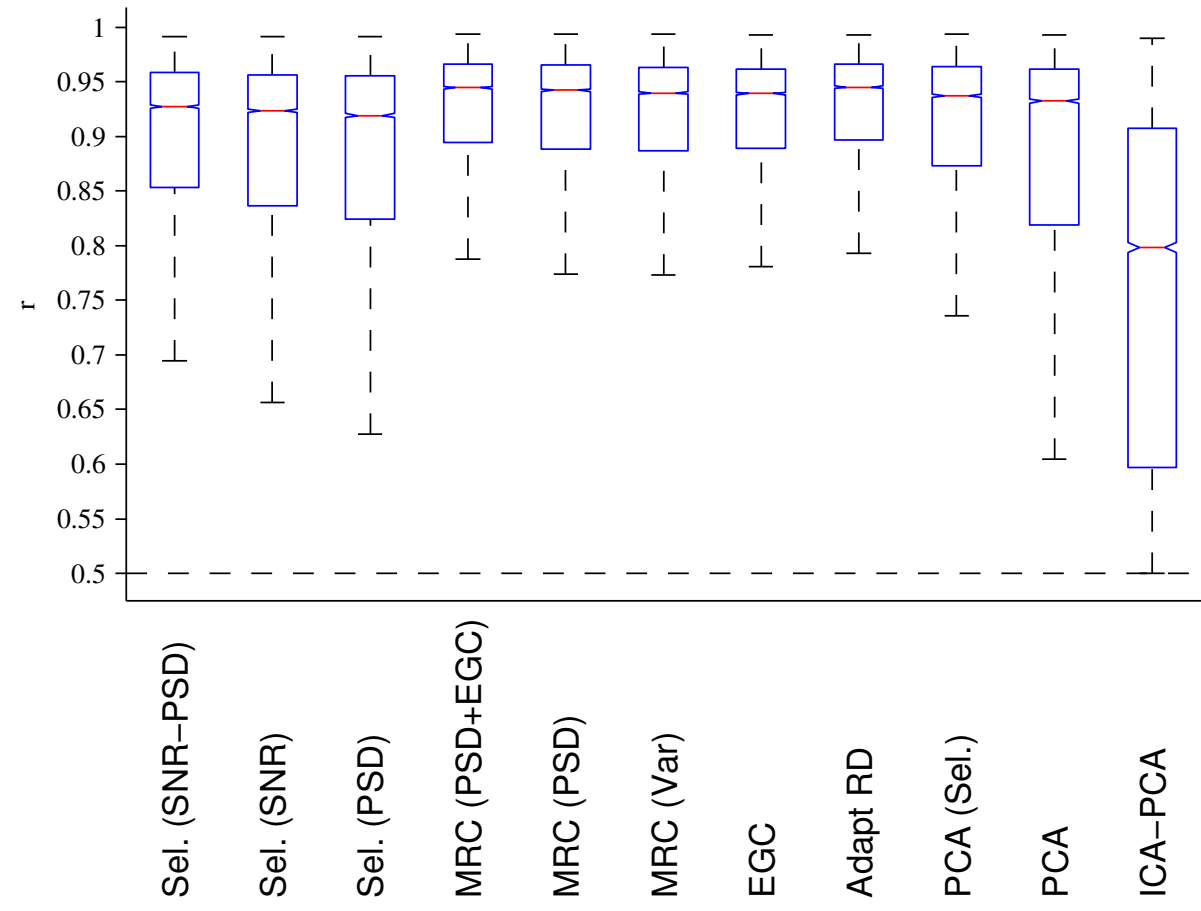

Figure 6.14: Boxplots of output signal quality by fusion method 


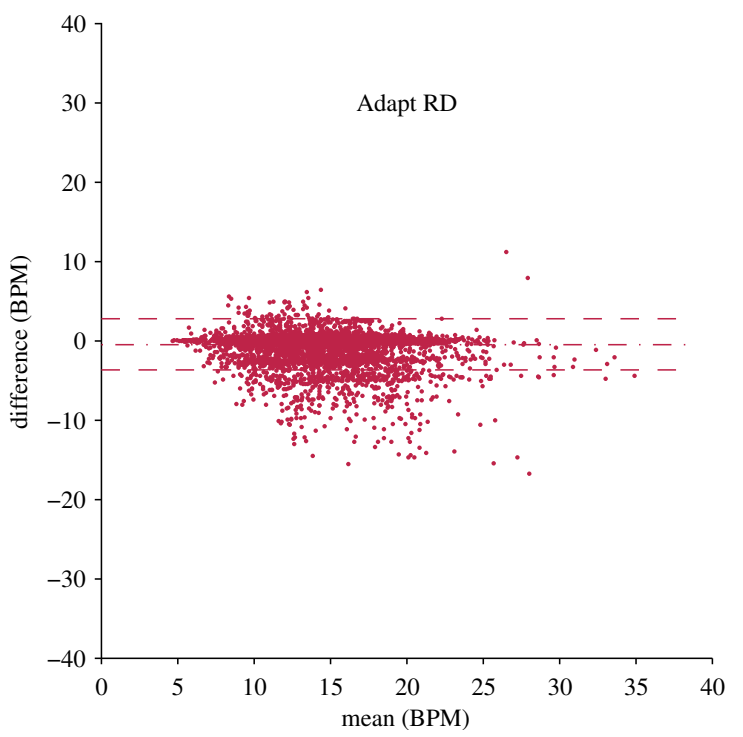

(a) Adapt RD

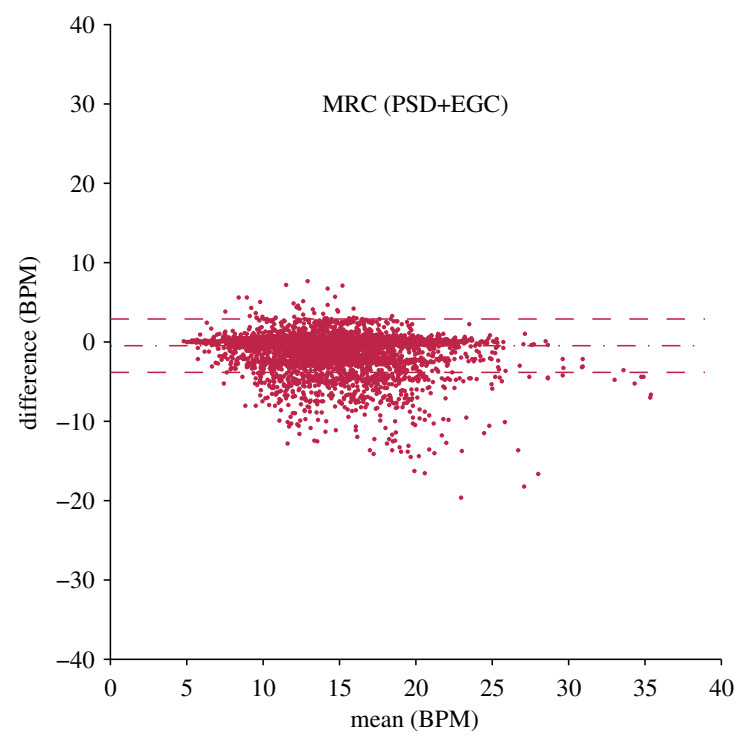

(b) MRC (PSD+EGC)

Figure 6.15: Bland-Altman plots of extracted respiration rates comparing respiband and ambient sensors for Adapt RD and MRC (PSD+EGC) methods

rejecting the null hypothesis of no improvement at $p=0.05$. The pairwise difference for $|\bar{r}|$ between Adapt RD and all methods was significant for all methods $(p<=0.001$ for all but MRC (PSD+EGC), which was at $p=0.002)$.

Fig. 6.15 depicts Bland-Altman plots for the respiratory rates calculated from the extracted signals. These results are very similar, but the Adapt RD method does display an advantage over the MRC (PSD+EGC) method at the highest respiratory rates, where all of the points higher than $30 \mathrm{BPM}$ lie inside its standard range. At lower rates, however, it seems to produce outliers that are a little further away from the center than the MRC (PSD+EGC) method does.

Table 6.2 compares the impact of respiratory rate extraction by participant for the Adapt RD method. Median absolute error was 0 for all participants. Variability between participants was again pronounced, with P49 producing over $90 \%$ of its epochs within 1 BPM, while P61 produced just below $72 \%$ within 1 BPM and was the only one with a mean absolute error above 1 BPM. On the other hand, P61 did 
Table 6.2: Statistics of respiratory rate estimation from signals fused using Adapt $\mathrm{RD}$, listed by participant

\begin{tabular}{|r|c|c|c|c|c|c|}
\hline & P49 & P61 & P82 & P89 & P95 & All \\
\hline \hline Median respiband rate (BPM) & 16.60 & 16.32 & 14.75 & 12.68 & 12.15 & 14.78 \\
\hline Median Adapt RD rate (BPM) & 16.73 & 16.90 & 15.03 & 13.17 & 12.27 & 15.18 \\
\hline \% not available & 0.21 & 0.39 & 0.89 & 0.55 & 1.32 & 0.65 \\
\hline Mean |error| (BPM) & 0.45 & 1.08 & 0.78 & 0.69 & 0.62 & 0.73 \\
\hline Median |error| (\%) & 0.61 & 1.02 & 1.05 & 1.50 & 1.05 & 0.99 \\
\hline Mean |error| (\%) & 3.33 & 8.17 & 5.79 & 6.36 & 5.75 & 5.88 \\
\hline \% |error| $<1 \mathrm{BPM}$ & 90.69 & 73.22 & 81.24 & 80.00 & 85.46 & 82.09 \\
\hline \hline
\end{tabular}

not have any epochs without discernible breaths.

\subsubsection{Discussion}

Most of the fusion methods compared here, except perhaps ICA-PCA, offered satisfactory results for combining breathing signals of the validation records. It is interesting to juxtapose the signal qualities of this experiment (Table 6.1) to those of the previous chapter (Table 5.1). Since the EGC results of this chapter and the DDSRD results of the previous chapter represent identical algorithms, the results can be compared. Although EGC was shown to be superior to selection combining here, when combined with less successful methods of reversal detection, such as spectral phase or correlation, its performance was inferior. This is perhaps why selection combining is a somewhat popular combining method for bed-based systems. Despite the fact that Adapt $\mathrm{RD}$ is in both tables, it represents slightly different algorithms. In the previous chapter it used only the sign of its weights. Making use of the magnitude of the weights improved $|\bar{r}|$ by 0.05 .

The improvements offered by the magnitude could also be seen in the rate results. 
The rate results can also be compared between chapters in Table 5.2 and Table 6.2. These tables are largely similar, but mean and median error both had incremental decreases. Mean error nudged lower from 0.74 to 0.73 . Another $0.1 \%$ of records fell within the 1 BPM error range, representing more than 550 more records within that range (of the $11000+$ records tested).

Breathing rate estimates using ambient systems can be quite precise. Kortelainen et al., used PCA to extract the respiratory intervals within an average error of just $1.5 \%$ [43], which is similar to the median error reported in table 6.2. Average errors here were larger, averaging almost $6 \%$, but this represented a $0.73 \mathrm{BPM}$ mean absolute error, which is lower than the error reported by Aubert and Brauers [27] of 1.25 BPM from a single wide area sensor $(30 \mathrm{~cm}$ x $60 \mathrm{~cm})$. Mack et al., working with a single differential sensor, reported breathing rate error as a standard deviation of the error, at 2.1 BPM. In this work, the standard deviation of the error was 1.65 BPM. The higher number of sensors that were used in Kortelainen's analysis (160 sensors) and here (48 sensors) would have likely improved the SNR of the breathing signal. Again, the improvement gained by increasing the numbers of sensors may be seen.

This chapter examined linear combining methods of data-level fusion, but nonlinear fusion methods could also be examined in future work. Wavelet signal fusion and EMD analysis are non-linear combining technique that may be suited to these non-stationary signals.

\subsection{Summary}

Three broad categories of ambient signal linear combining for breathing signal fusion were analyzed: sensor selection, diversity combining, and blind source separation. To this end, spectral estimations of signal and noise power were detailed and used in new methods in all three broad categories. Eleven methods of linear combining 
were compared. The methods that performed best for fusion of real-life breathing signals and that were most resilient to simulated signal degradations were a diversity combining method, maximal ratio combining (MRC (PSD+EGC)) and a blind source separation method. The latter was based on the adaptive method of reversal detection developed in the previous chapter, Adapt RD. These two methods provided an overall better estimate of the breathing signal. By applying Adapt RD here, mean respiratory rate error fell another $5 \%$, compared to the best fusion results of the previous chapter. However, in specific scenarios other algorithms performed better. For instance, sensor selection and principal component analysis methods performed well when few sensor signals carry available breathing signals. Furthermore, these two methods did not require a separate reversal detection stage prior to fusion. Reversal detection can make or break the benefits derived from diversity combining algorithms; it was revealed that sensor selection performs better than these methods when reversal detection is poor. 


\section{Chapter 7}

\section{Ensemble Fusion by Environmental}

\section{Context Awareness}

In the previous chapter, a number of fusion methods were analyzed towards extraction of quasiperiodic signals from ambient sensors. Although some methods were generally better than others, there was no free lunch: no method always produced superior

results. Depending on the interference and intrinsic signal conditions present with the input signals from the sensors, one method might produce superior results in one situation but poor results in another. For example, the proposed MRC (PSD+EGC) method performed better than other methods when movement artifact was present in the signal, but saw diminished performance when few sensors carried the breathing signal.

This chapter proposes to take advantage of this diversity of performance and tap into each method's strengths. By using an ensemble of fusion methods and selecting the best method for the given conditions, a more robust system is possible. A manuscript of this work, albeit using a linear classifier rather than the SVM presented here, has been accepted for publication in [56]. 


\subsection{Introduction}

Many sources of signal degradation and interference exist in the ambient sensor environment. Fig. 7.1 displays some of the many environmental and signal factors that can affect the input signal from sensors and ultimately impact the fusion performance. These include factors related to the sensors themselves, such as noise and

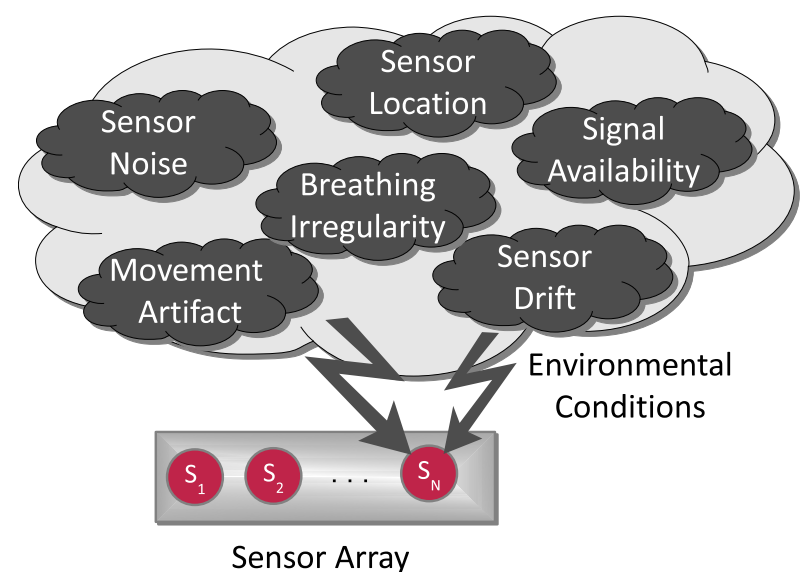

Figure 7.1: Environmental conditions affecting fusion methods and its output signal quality

drift, factors related to the location of the sensors, such as signal availability, factors related to interference from other signals in the environment, and factors related to the real-world physical signals to be extracted, namely non-stationarity in its period (quasiperiodic behaviour), irregular signal shapes, and changing amplitudes.

If the optimum method for every epoch was known a priori, significant improvements could be made to the combining performance. However, the choice can only be made by examining the factors and choosing the expected best. The problem becomes not only how to make use of multiple fusion methods, but also how to estimate the conditions present in the data due to the environmental situation of each sensor and the intrinsic signal properties. The sensor signals themselves must be mined to provide this information. 
The proposed method mines the sensor signals to provide the environmental context. The proposed method of ensemble signal fusion using environmental and signal context is outlined in Sec. 7.2, including a description of the fusion blocks, the contextual features, the classifier, and the training procedure. Optimization of the classifier is then described in Sec. 7.3.

\subsection{Method: Ensemble Signal Fusion using Envi- ronmental and Signal Context}

Ensemble methods allow many weak methods to achieve superior performance, as long as there is diversity among them [172]. Generally, ensemble systems refer to the use of multiple classifiers. Ensemble methods have been used for fusion tasks, but these have been for feature level fusion [173]. Here, a single classifier is implemented with multiple combining/fusion methods to perform data-level fusion.

The block diagram of the proposed contextual selection system is depicted in Fig. 7.2, making use of an ensemble of fusion methods. A "fusion agent" is presented as

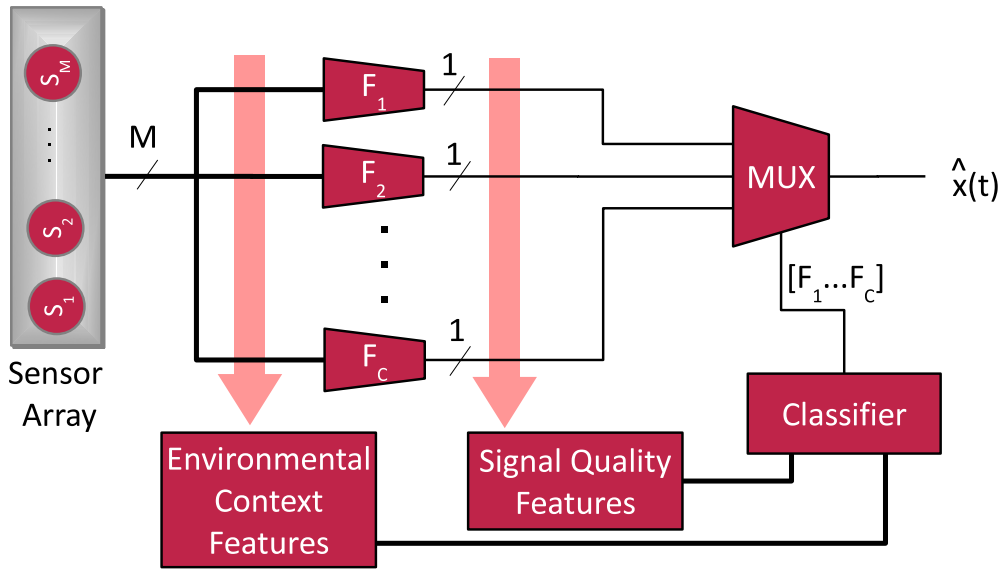

Figure 7.2: Block diagram of ensemble fusion using environmental and signal context

an individual fusion block which takes $M$ inputs from the sensor array, applies signal 
conditioning that is appropriate for its fusion method and outputs its representation of the original signal. These are labeled $F_{1}, F_{2} \ldots F_{C}$ in the diagram. The output signals from each of the fusion agents are in turn fused using a selection based method. Rather than maximizing a fitness function to select the fusion agent, a trained classifier selects the most appropriate one, given environmental context features and signal quality features.

The candidate fusion agents used in this work are the 11 fusion methods appearing in the previous chapter.

\subsubsection{Contextual Features}

To estimate the conditions present in the data due to the environmental situation

of each sensor and the intrinsic signal properties, features can be extracted from the sensor signals. To provide information regarding the environmental conditions of interference, the following features were calculated:

move\%: Percent of the record with detected movement

maxSNR: maximum estimated signal to noise ratio (SNR) in $\mathrm{dB}$

meanSNR: mean estimated SNR in dB

meanrange: mean range of the signal in the record

maxSDR: maximum estimated signal to drift ratio (SDR) in $\mathrm{dB}$

meanSDR: mean estimated SDR in $\mathrm{dB}$

Maximum and mean values refer to statistics collated across all input sensors. To provide information regarding the signal quality, the following features were extracted:

SNR: Mean output SNR in dB 
mri: Mean interval between breaths detected in the record

sri: Standard deviation of the interval between breaths detected in the record

nsri: Standard deviation of the interval between breaths detected in the record, normalized to the mean interval

These signal quality features were extracted from the output of each fusion agent, so for every record, there are $C$ mri and $C$ sri features. All of these features were chosen as being non-specific to a given array size or orientation.

Scaling of features is an important consideration, as most classifiers prefer normalized features to unbounded values. This keeps any one feature from overpowering the others and standardizes the inputs. Scaling was performed to a $[0,1]$ range, by subtracting the minimum value and dividing by the total range. There were a few outlying SNR values, and so SNR was clipped from -15 to $+50 \mathrm{~dB}$ before scaling. Scales were determined from the training set, without knowledge of the scales of the test data. It is therefore possible for test data to go beyond the $[0,1]$ range.

\subsubsection{Classification}

The approach outlined here is independent of the type of classifier used. To achieve good results, the chosen classifier must be able to generalize the problem, given input training samples. In this work, an support vector machine (SVM) classifier is used. Comprehensive tutorials on SVM classifiers are available in [174] and [175].

SVM classifiers divide the data space using hyperplanes, somewhat like linear classifiers. Unlike linear discriminants, they maximize the margin between the classification boundary and the training points nearest to it [174]. These training points are called the support vectors. For datasets without clean boundaries, that are nonseparable, a cost parameter is added to allow misclassifying some samples while 
minimizing the cost [176]. SVM's provide generally accurate classification performance [175]. Although they are based on linearly separation, a kernel function provides non-linear mapping so that they may be used to classify non-linear boundaries $[176]$.

\subsection{Optimization}

It is important to avoid the "curse of dimensionality". That is, although additional agents or features provide more information to the classifier about the task at hand, higher complexity makes it easier to overtrain the classifier. Overtrained classifiers represent the specific trained set, but do not generalize the problem. Reducing the number of classes to discriminate helps to reduce complexity and improves general classification accuracy.

To acheive good results, the classifier must be able to generalize the problem, given the input training samples. To achieve good generalization, without overfitting the data, regularization is required. In this section, we outline methods of regularization that were applied to the problem.

\subsubsection{Fusion Agent Optimization}

Selecting only the best fusion agents for inclusion in the classification task reduces the complexity of classification. This can be seen by considering a random classifier. Let $C_{\text {all }}$ be the total number of fusion agents available. If the prior probability of any fusion agent being the best in a given situation are equal between agents, the

probability of randomly selecting the correct one is $\frac{1}{C_{\text {all }}}$. If only $C$ fusion agents are used instead, where $C<C_{\text {all }}$, the classification accuracy increases. However, it is important that the $C$ fusion agents are selected wisely. Classification accuracy may increase, but if the classifier is choosing among $C$ poor fusers, output fusion 
performance is limited by their fusion ability.

To optimize the fusion agents, agents were selected sequentially according to their performance on training data. First, the agent that had the best overall performance was chosen. Then, one at a time, candidate agents were evaluated for addition to the set of selected agents by five-fold cross-validation, including only the selected agent set and the candidate agent. After every agent was evaluated, the agent that resulted in the highest performance was selected and added to the set of selected agents. At the next iteration, only the remaining agents were evaluated. This continued until all candidate agents were ordered. Concurrently, the best possible performance possible using only the selected agents was evaluated as the optimal result in addition to the performance of a five-fold uniform random selection. This latter provided a lower bound of performance while the former was an upper bound. Since classifier accuracy will tend to fall with increased output classes, the output signal quality was used to judge performance rather than classification accuracy. Once agents were ranked, the resulting performances were evaluated and the top agents were selected.

\subsubsection{Classifier Optimization}

When training an SVM, there are two main areas of optimization: the kernel choice and the associated parameters. The first is choosing the kernel function that to remap a linear space into a non-linear separation. A radial basis function (RBF) is a practical default choice [177], although other choices such as a polynomial or sigmoidal functions are also popular. RBFs only require tuning a single parameter, gamma. $\gamma$ determines the radial width, which decreases as $\gamma$ increases:

$$
k(x, y)=e^{\left(-\gamma\|x-y\|^{2}\right)} .
$$


This parameter must be optimized, alongside the "cost" parameter. As outlined in Sec. 7.2.2, the cost is a weighting given to misclassified points in non-separable data. By judiciously tuning these parameters for the desired application, good generalization of the training data occurs and leads to an accurate classifier.

A grid-search method was used to tune the parameters. Pairs of $\gamma$ and cost were set along a grid and five-fold cross-validation was used to estimate the performance of the classifier according to both accuracy and mean output signal quality. Values of $\gamma$ ranged exponentially (base 2) from 0.125 to 16 , while cost ranged exponentially (base 2) from 1 to 256.

\subsection{Experiment}

To test the hypothesis that an ensemble of fusion agents can provide better fusion, an experiment was run using all of the fusion agents presented in the previous chapter.

Training data sets were created from the simulated corruption records including noise, drift, and reduced signal availability, as well as from the main training set of uncorrupted records. To balance the dataset, the same number of records was retrieved from each set using uniform random sampling. 6500 records were selected by uniform random sampling, without replacement, from each set. A total of 26 000 records were available for training. To allow for testing with corrupted data, the training data was split into two along a randomly sampled 90\%/10\% split. The larger set of 23400 records remained for training, while the smaller set of 2600 records was left for final testing.

Testing was performed in MATLAB using LIBSVM, an open source library for SVM classification with a MATLAB interface [178]. Due to the large number of records in the training data and the time required to train a classifier, a subset of records (5000) was randomly selected for fusion agent selection and parameter 

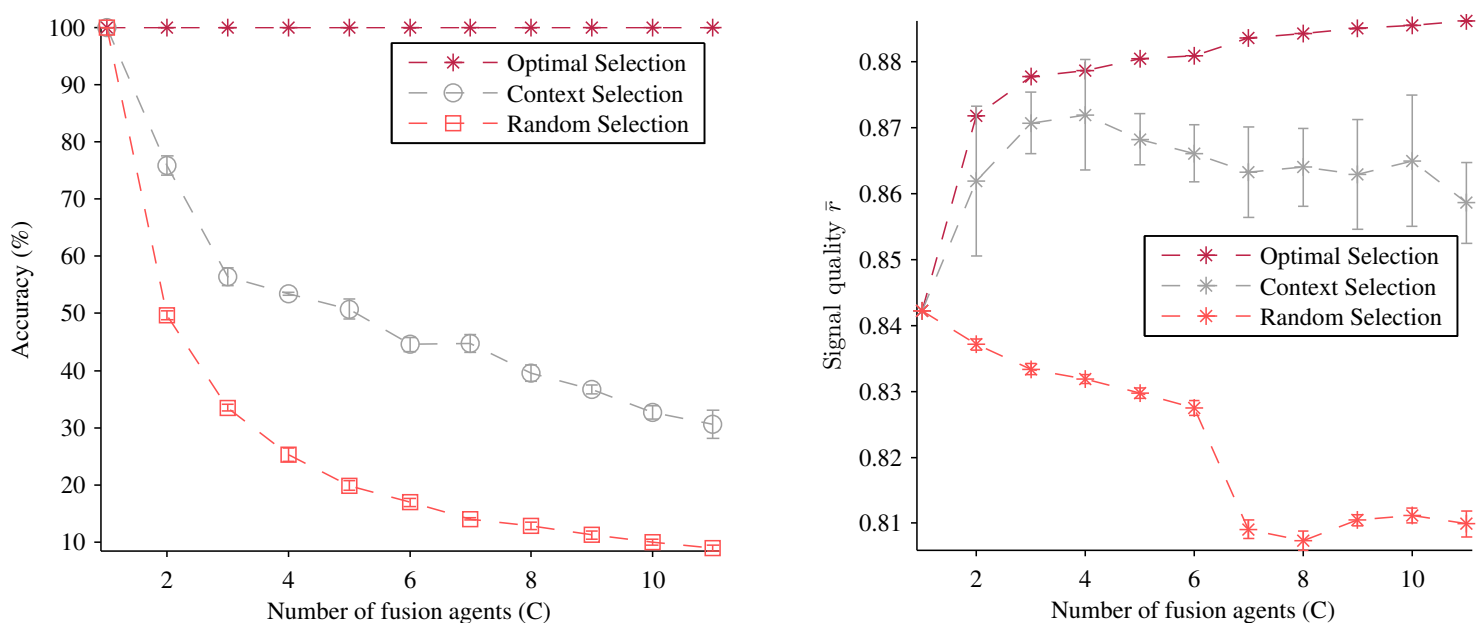

(a) Classification accuracy as a function of the (b) Output signal quality as a function of the number of fusion agents

number of fusion agents

Figure 7.3: Classification accuracy drops as the number of fusion agents increases, but output signal quality improves - up to a point.

optimization.

As in the previous chapters, data from the five participants not included in the previous data was used for a final test. In addition, the data from the multi-mattress experiment was revisited and the results with that set were also reported.

\subsubsection{Results}

\section{Fusion Agent Optimization}

For every added agent, classification accuracy drops as there is more probability of a selection error. Even so, the quality of the final output signal need not decrease and may improve, even with poorer classification performance. Fig. 7.3 shows how classifier accuracy drops as fusion agents are added to the ensemble yet signal quality initially rises. The classifier provides signal quality performance that far exceeds the random selection, but still has room to improve towards optimal selection. The drop of random selection accuracy at $C=7$ can be attributed to adding the ICA-PCA 
classifier, which has generally poor fusion performance, but does provide the best option in a handful of records.

Once the complexity of the classification task is too high, with too many added agents, signal quality decreased. $C=4$ was selected as the number of agents to use. It could be argued that given the confidence intervals overlap, three would also be a valid choice. Error bars based on the variance of errors on cross-validated data are invariably biased [179]. The error bars shown represent the standard deviation of the cross-validation evaluation, which does not take into account the number of samples tested for each fold and may overstate the interval. The selected agents were, in order of selection (agent label $c$ in brackets): "MRC (PSD+EGC)"(3), "Sel. (PSD)"(1), "Adapt RD"(4), and "EGC"(2).

Table 7.1 breaks down the fusion agent optimization by signal quality performance

$|\bar{r}|$, the percentage of the records in the set that a given method was the best, the ranking of the fusion methods, and the labels $c$ given to the chosen methods (a label of 0 indicates the method was not selected). The qualities listed here differ from the quality of the methods in the validation data of the previous chapter (Table 6.1), since this dataset emphasizes poor conditions. The rankings do not align with the listed quality since the best results are achieved when agents cover diverse conditions. For example, MRC (PSD) provides better performance than Sel. (PSD), but is likely to perform well in the same conditions that MRC (PSD+EGC) does. The first three selected methods were each from the three major categories of combiners: weighted summation combiners, selection combiners, and blind source separation combiners.

\section{Parameter Optimization}

Fig. 7.4 shows the effect of the cost and $\gamma$ parameters on classification accuracy by displaying the results of the grid-search. Classification performance was generally poorer for higher values of cost and $\gamma$, however this pattern did not extend to the 
Table 7.1: Evaluation of fusion agents from training data: general signal quality, percentage of records as top agent, computed ranking, selection label, and percentage of records as top agent among selected agents

\begin{tabular}{|r|c|c|c|c|c|}
\hline & $|\bar{r}|$ & \% Best & Rank & $c$ & Top \% Best \\
\hline \hline Sel. (SNR-PSD) & 0.824 & 1 & 5 & 0 & 0 \\
\hline Sel. (SNR) & 0.794 & 7 & 11 & 0 & 0 \\
\hline Sel. (PSD) & 0.832 & 17 & 2 & 1 & 26 \\
\hline MRC (PSD+EGC) & 0.841 & 17 & 1 & 3 & 37 \\
\hline MRC (PSD) & 0.826 & 8 & 10 & 0 & 0 \\
\hline MRC (Var) & 0.807 & 8 & 6 & 0 & 0 \\
\hline EGC & 0.826 & 4 & 4 & 2 & 9 \\
\hline Adapt RD & 0.823 & 12 & 3 & 4 & 27 \\
\hline PCA (Sel.) & 0.828 & 15 & 9 & 0 & 0 \\
\hline PCA & 0.794 & 7 & 8 & 0 & 0 \\
\hline ICA-PCA & 0.702 & 4 & 7 & 0 & 0 \\
\hline
\end{tabular}

signal quality. The best region was to keep the parameters in a mid-range, and extend one of them further than the other. Selected parameters were the pair generating the best signal quality, with cost $=18.5$ and $\gamma=1.25$.

Fig. 7.5 shows that optimizing for classification accuracy directly increases output signal quality, although in some areas of the grid, a bifurcation occurred. This could be because the classifier was increasing accuracy between the top methods, where signal quality differences were small, at the expense of letting some of the records with larger quality differences be misclassified. 


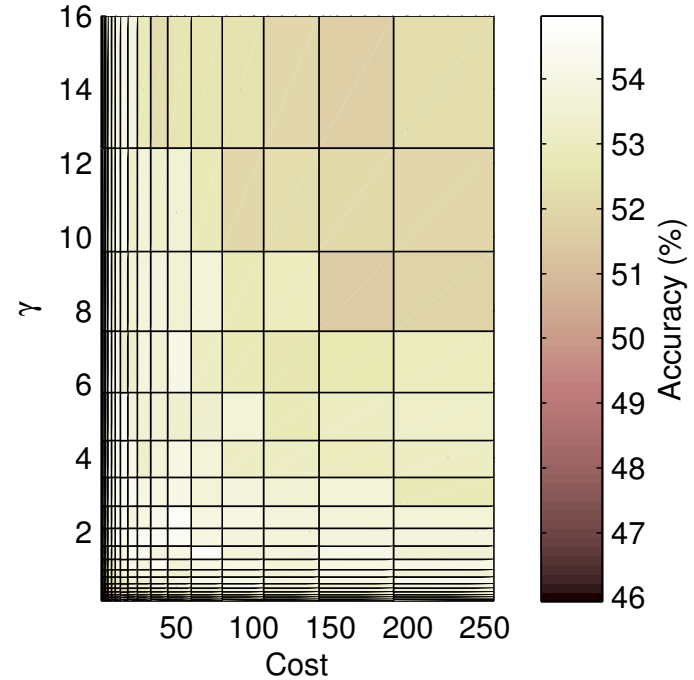

(a) Classification Accuracy

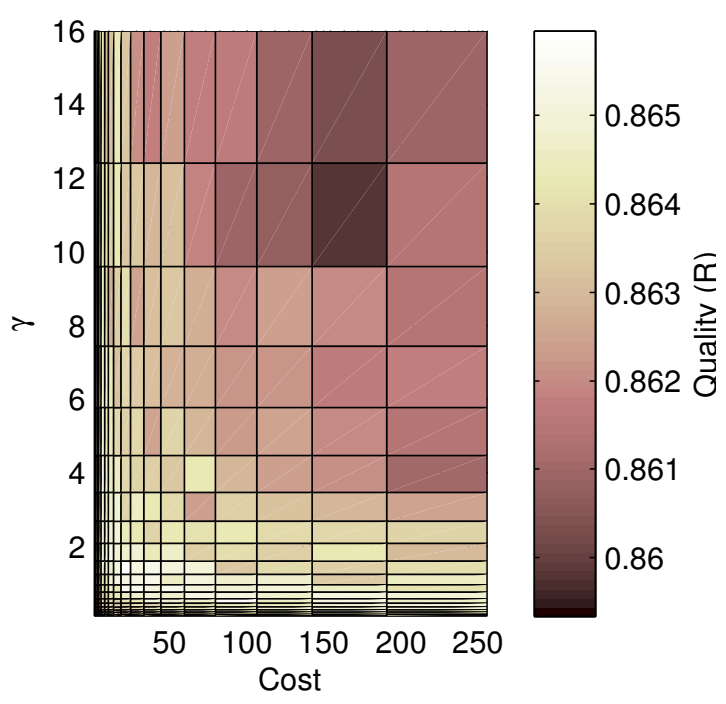

(b) Output signal quality

Figure 7.4: SVM parameter selection: Classification accuracy and signal quality according to cost and $\gamma$

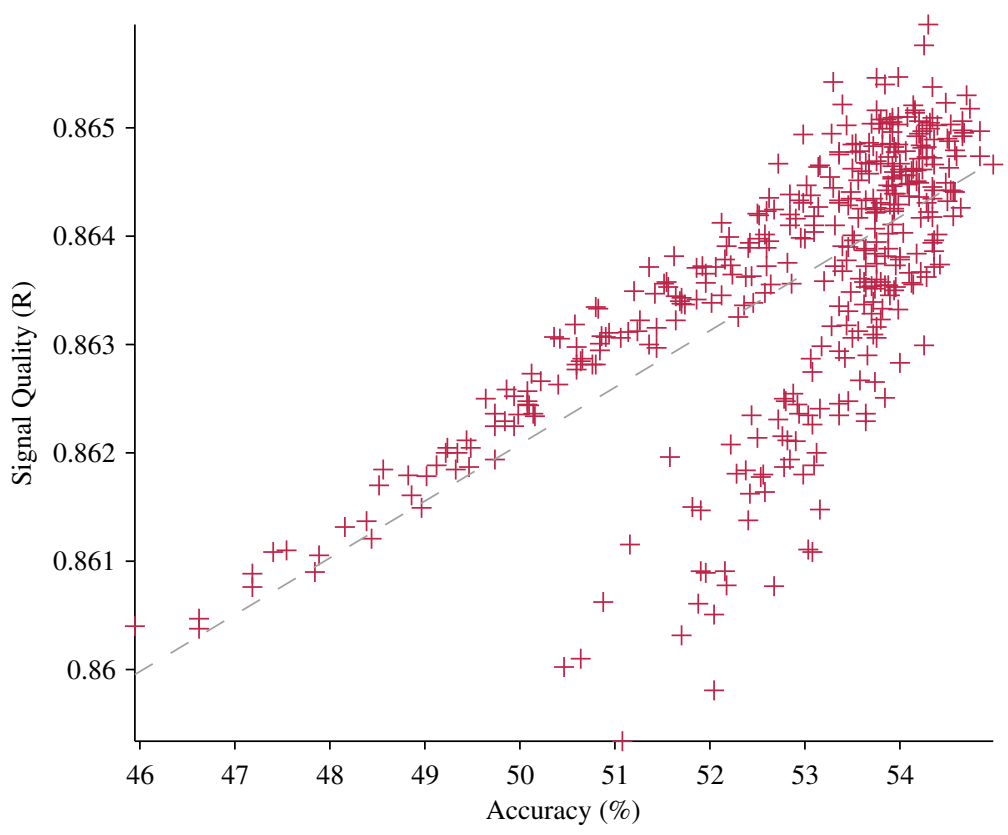

Figure 7.5: Output signal quality with respect to classification accuracy: signal quality rises with classification accuracy 


\section{Test and Validation Performance}

Table 7.2 displays the results of testing classifiers trained with the full training data on the test sets. The synthetic test set refers to the $10 \%$ of training records set aside for final testing and includes synthetic corruptions. Multi-participant refers to the validation data set from the last five participants of the multi-participant experiment, also used for testing in the previous chapters. Optimal methods and random choice methods are also shown for comparison. Finally, Adapt RD and MRC (PSD+EGC) are also included to show the improvement gained by ensemble fusion over picking just one of these. The random classifier results were from random selection of the four selected agents, iterated 100 times over the test sets. The contextual method shows

Table 7.2: Performance of fusion methods applied to the test sets

\begin{tabular}{|c|c|c|c|c|c|c|}
\hline & \multicolumn{2}{|c|}{ Synthetic } & \multicolumn{2}{|c|}{ Multi-Participant } & \multicolumn{2}{|c|}{ Multi-Mattress } \\
\hline & (Acc. \%) & $(\mathrm{R})$ & (Acc. \%) & $(\mathbf{R})$ & (Acc. \%) & $(\mathbf{R})$ \\
\hline Optimal & 100.0 & 0.884 & 100.0 & 0.909 & 100.0 & 0.897 \\
\hline Context & 55.2 & 0.872 & 40.0 & 0.893 & 41.2 & 0.877 \\
\hline Adapt RD & 26.7 & 0.831 & 30.8 & 0.892 & 18.4 & 0.870 \\
\hline $\mathrm{MRC}$ & 36.7 & 0.849 & 35.6 & 0.889 & 40.2 & 0.875 \\
\hline Random & 25.4 & 0.839 & 24.6 & 0.879 & 24.9 & 0.863 \\
\hline
\end{tabular}

large improvements over choosing either MRC or Adapt RD for the test set with added synthetic corruption. It is able to correctly select the best fusion agent for each record over $50 \%$ of the time. In this set, Adapt RD shows poorer performance than random selection as the inclusion of drift records degrade its results and the other agents provide better performance. For the multi-participant experiment, the contextual ensemble method provides $10 \%$ better accuracy than selecting Adapt RD, which leads to an incremental improvement in signal quality, and edged up the percentage 
of good quality records $(|\bar{r}|)$ from $90.7 \%$ to $90.8 \%$. The multi-mattress experiment had MRC (PSD+EGC) as the single top method rather than Adapt RD. Again, the contextual ensemble was able to depend on the known conditions and select better agents.

Fig. 7.6 shows the confusion matrices for the SVM output, comparing the selected fusion agent to the target agent. From the confusion matrices, it can be seen how

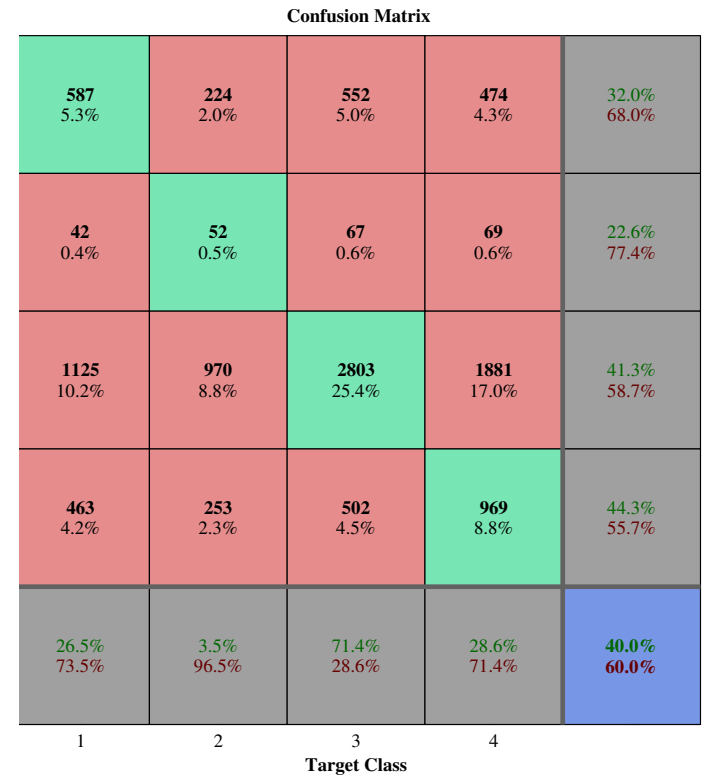

(a) Multi-Participant

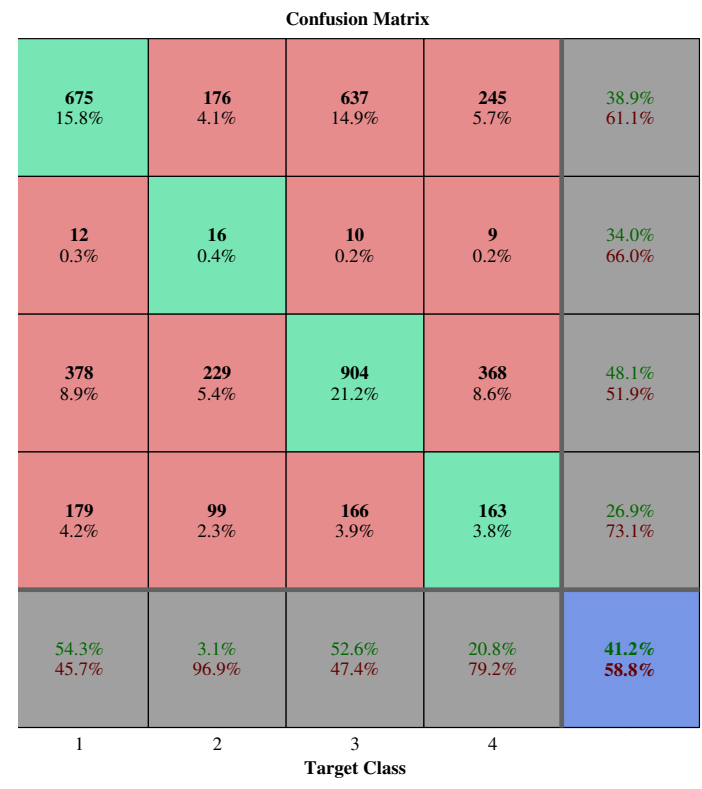

(b) Multi-Mattress

Figure 7.6: Confusion matrices for selected agents performance over the test sets. The columns indicate the target agents and the rows the output agents. The diagonals represent the true positives. Ordered from left to right, top to bottom: 1. Sel. (PSD), 2. EGC, 3. MRC (PSD+EGC), 4. Adapt RD. The grey row along the bottom and column on the left represent totals.

the contextual classifier chooses MRC (PSD+EGC) and Adapt RD more often for the multi-participant experiment, but Sel. (PSD) and MRC (PSD+EGC) more often for the multi-mattress experiment. There are still more incorrect selections rather than correct ones. Given the overlapping data present in the sets data, it perhaps 
not surprising that it is not always able to choose the optimum value. For falsely classified data, it is possible that the optimum agent for a particular record is not measurably better than the selected agent.

Table 7.3 compares the impact of respiratory rate extraction for the context method between all three experimental test sets. Compared to the multi-participant

Table 7.3: Statistics of respiratory rate estimation from signals fused using ensemble fusion with contextual awareness for the multi-participant experiment

\begin{tabular}{|r||c|c|c|}
\hline & Synthetic & Participant & Mattress \\
\hline \hline Median respiband rate (BPM) & 15.35 & 14.78 & 14.07 \\
\hline Median context rate (BPM) & 15.42 & 15.13 & 14.46 \\
\hline$\%$ not available & 4.19 & 0.41 & 0.52 \\
\hline Mean |error| (BPM) & 0.61 & 0.71 & 1.02 \\
\hline Median |error| (\%) & 0.85 & 1.01 & 1.48 \\
\hline Mean |error| (\%) & 4.52 & 5.69 & 10.48 \\
\hline$\%$ |error| $<1 \mathrm{BPM}$ & 88.48 & 82.82 & 77.26 \\
\hline
\end{tabular}

results in the previous chapter, mean BPM error has nudged down by $2.8 \%$ from 0.73 (Table 6.2) to 0.71 , although median error as a percent of the respiratory rate has increased by $2.0 \%$. The percentage of epochs with error less than 1 BPM has nudged up. The number of epochs without detectable breathing has fallen, from $0.65 \%$ to $0.41 \%$, representing just over 25 more epochs from the $11000+$ records. The multimattress experiment has a larger mean absolute rate error than the multi-participant experiment, but $1.02 \mathrm{BPM}$ still represents an improvement over both Adapt RD at 1.06 BPM and MRC (PSD+EGC) at 1.15 BPM and provides 1.5\% more epochs with rates within $1 \mathrm{BPM}(77.3 \%$ vs. $75.7 \%$ and $75.4 \%$ respectively). 


\subsubsection{Discussion}

As with other ensemble methods, the ensemble of fusion agents was able to outperform the best single agent. By selecting between agents based on contextual information mined from the sensor inputs and the agent outputs, output signal quality improved in difficult conditions. Creating features that provide good clues regarding the possible conditions in the data is essential to success. The features that were chosen here were able to supply the classifier with the information required to make an informed decision for many ambient conditions and signal characteristics. Even in the relatively clean data of the multi-participant and multi-mattress test sets, the classifiers encountered conditions that required each of the fusion agents to be selected at least once. Furthermore, the fusion agents that worked best for each of these sets were different and the contextual classifier was able to improve over the best constant method in both of these situations.

The use of an SVM, a relatively advanced classifier, performed better than a simpler linear classifier. The feature sets showed that the classification boundaries were not linearly separable and by using the SVM, non-linear boundaries were created. Initial testing using a linear classifier showed that performance for corrupted data was also improved, but by a smaller margin. Furthermore, performance for less-corrupted sets, such as the multi-participant and multi-mattress sets were not improved over the top methods, although fusion performance was still generally good, matching at least the second-best method.

Optimization of the classifier was performed to select top fusion agents and good parameters. Further optimization could improve results, such as studying whether a different kernel is more appropriate or doing feature selection. SVM classifiers do not suffer as much as other classifiers might from the "curse of dimensionality" since their use of maximized margins helps protect against overfitting and they use a simple linear 
decision rule [174]. However, overfitting can occur by choosing the SVM parameters poorly and some feature selection has been shown to offer improvements [180]. In the feature set used here, there was some overlap in the features that may be unnecessarily complicating the classification without offering additional information. In particular, the nsri feature is a combination of the mri and sri features. Even the optimizations that were performed could be improved since exhaustive searches were not performed. A particle swarm method of simultaneous feature selection and parameter selection could find a more optimal combination [181]. A feature that was not used here, but that could offer insight into environmental conditions is the posture of the bed occupant, inferred from the data through a posture classification algorithm. Assessing and improving the accuracy of the features themselves when data corruption is present may also realize a more robust system.

The final trained classifier is sensitive to the corruptions present in the training data. It was found during fusion agent optimization that the selection of fusion agents depended on which data sets were presented during training. For instance, if movement data from the base training set was not present during training, the system did not learn that this interference was important and disregarded the fusion agents that worked better during movement. Additionally, when the number of training points for one type of condition was reduced compared to other conditions, the classifier was less likely to optimize for that condition. An equal number of training points from each type of condition is preferable. However, if the application is expected to suffer from one condition more than others, it could be useful to weight the training set towards that condition.

In this work, the fusion of fusion agent outputs was accomplished using a selection method. It would be possible to use a weighted output method so that the final output is a weighted combination of the fusion agents' outputs. In the previous chapter, weighted combining was shown to perform generally better than selection combining. 
For example, an ensemble of regression agents could be used to learn the expected signal quality for the conditions and the output predicted quality used to weight the fusion.

The ensemble method was used here to choose the best fusion agent, but it could also be used in a similar manner on the methods presented in Chapter 5, to choose an optimal reversal detection method. This could be used to improve reversal detection and the fusion methods that rely on this detection, namely the diversity combining methods of MRC and EGC.

When signal quality is not paramount, the implementation of multiple fusion methods and a classifier may be too complex. However, given the medical application, where critical health decisions may be made based on the extracted breathing signal, added complexity may be justified. The issue of what is "good enough" arises. While the mean signal quality improvement is assessed here, it is the switchover of epochs from poor to adequate signals that reduce the possibility of making bad medical decisions. Every additional 1\% of epochs with good signal quality translates into an extra five minutes a night of solid monitoring. For the difficult conditions present in the test data here, contextual selection supplied respiratory rates within 1 BPM of the respibands for $2 \%$ more of the night. While this might not represent a large portion of the night, it may be that this portion contains the most salient information, since difficult fusion conditions go hand-in-hand with disordered breathing. Not only may this extra monitoring time provide salient information that is missed otherwise, but it also reduces the burden of detection of poor epochs. A poor signal may be worse than no ambient monitoring at all if the poor signal quality is not recognized and medical decisions are made from the poor signal. For example, misdetecting breaths from interference peaks while apneas are actually present could provide a false negative for sleep disordered breathing and screen a patient away from receiving sleep medicine care. 


\subsection{Summary}

An ensemble of fusion methods, or fusion agents, was assembled, selecting between each possible fusion agent given the conditions present in the input and output signals. Ranking and selecting fusion agents resulted in four agents chosen, with at least one from each of the general categories of agents presented in the previous chapter. The ensemble-based context selection method was able to more accurately fuse corrupted records than the top fusion agent, MRC (PSD+EGC), providing the best signal quality possible in over $50 \%$ of the test records, compared to just over $35 \%$ for $\mathrm{MRC}$ $(\mathrm{PSD}+\mathrm{EGC})$. For the multi-participant and multi-mattress experimental data, it was able to select the best algorithm $40 \%$ of the time, and nudged up signal quality and the number of records with less than $1 \mathrm{BPM}$ error for both. While the increase was minimal, the best pre-selected method for each set varied. With contextual selection, results were consistently good across all sets. When conditions are expected to be good, similar to laboratory conditions, it may not be worth the added complexity to

implement an ensemble fuser. However, for medical applications and in scenarios of unknown signal conditions, it provides a more robust system. 


\section{Chapter 8}

\section{Applications in Clinical Care}

This chapter applies the ambient monitoring system to observing actual patients, either at home or in an institution. The incorporation of ambient technology into clinical care is discussed, with emphasis on two potential clinical applications: cognitive function, and palliative care. The application in palliative care is further developed in two pilot studies with participants from the Palliative Care Ward at Elisabeth Bruyère Hospital. The first study examined patient motion, where researchers watched and recorded the movements of palliative care participants around the clock. These annotations could be compared directly to motion information extracted from collected sensor data. The second study performed long-term breathing analysis. Breathing signals were extracted from palliative care participants, with comparison to overnight data from healthy participants.

Preliminary long-term breathing analysis of palliative care patients was published

in [57] and [182]. Apnea detection via the pressure variance appears in [59-61]. Preparatory work regarding incorporating ambient technology in the home appears in $[58,62]$. 


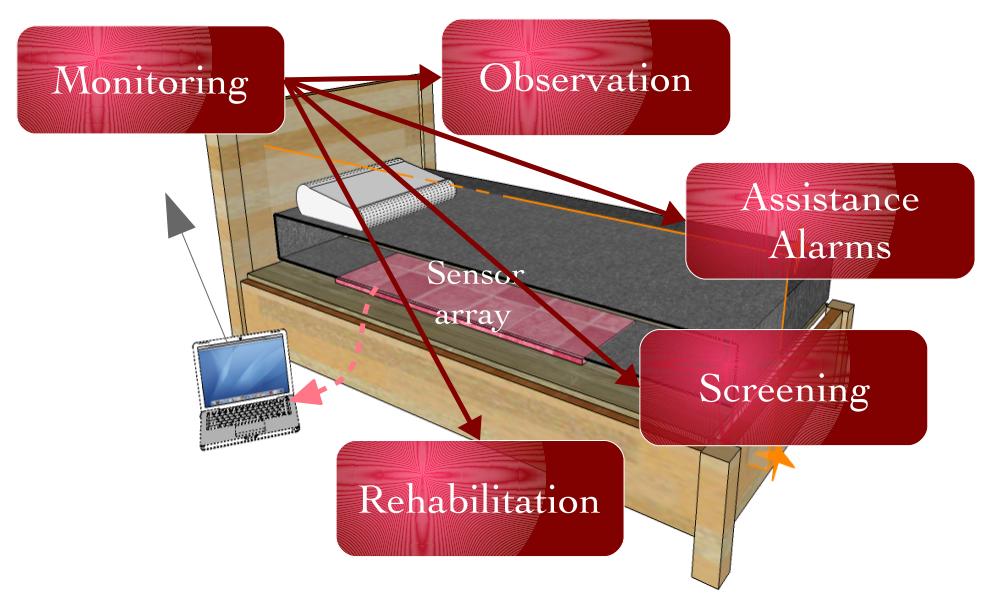

Figure 8.1: A sensorized bed for ambient care

\subsection{Incorporating Ambient Technology into Clini- cal Care}

Bed-based sensing is a natural medical monitoring fit. Benefits can be realized for both home care and institutional care, since beds are common to homes, hospitals and other institutions. Healthy individuals spend almost one third of their lives in bed [183], and as healthy people fall sick, they spend a larger proportion of their day in bed. Ambient health monitoring offers the potential for long-term observation, screening, rehabilitation, and even alarm activation for assistance, as depicted in Fig. 8.1.

Sleep medicine has understandably been a primary target application for researchers working with bed-based ambient systems. However, two overlooked fields of clinical care that could benefit from ambient systems are cognitive medicine and palliative care. The role of ambient technology for each of these clinical care fields, and specifically how the bed-based sensor system could be incorporated, is detailed in the following sections. 


\subsubsection{Applications in Cognitive Medicine}

Wai et al. proposed monitoring of cognition impaired adults with a pressure sensing bed [71] in order to prevent bed sores from lying in bed too long. However, much more is possible in this field, both for monitoring mild cognitive impairment (MCI) and dementia.

MCI has been receiving attention in recent research because it is an intermediate state between normal healthy aging and dementia, such as Alzheimer's disease [184]. Capturing the conversion between the states of MCI and dementia is critical for prompt initiation of medication, thus slowing the progression of cognitive decline. Current monitoring of patients with MCI is infrequent, typically with a six-month to one-year follow-up. This check up includes a short cognitive test, but does not capture subtle changes that can occur over the period. An ambient monitoring system has the potential to capture many of these changes. These include changes in sleep architecture, sleep disturbances, and impairment of lower limb function.

Alterations in sleep architecture are characteristic of cognitive impairment. These changes include five behaviours: nighttime wandering, circadian rhythm disturbances, increased sleep latency, increased sleep duration, and increased daytime napping [185]. Sensorized beds have often been applied to studying sleep architecture to capture all of these behaviours, using either simple bed occupancy monitoring or more sophisticated sleep staging techniques $[37,39,40,95,126-128,186]$.

Sleep disordered breathing is a sleep disturbance that has been associated with cognitive impairment [187], [188]. This thesis has demonstrated the feasibility of monitoring breathing signals with ambient bed-based systems, while apnea-specific events have also been detected in related research [189] and other clinical research $[34,42,129,130]$. Another sleep disturbance related to cognitive impairment is restless leg syndrome, which is associated with nocturnal agitation behaviours in patients 
with dementia [190]. Periodic leg movements such as those the comprise restless leg syndrome have been detected with bed-based systems [131].

Not only can restless legs be detected, but impairment of lower limb function can also be observed. Limb impairment can be both predictive of future cognitive decline and dementia [191], [192], and increases the probability of early Alzheimer's disease compared to MCI [193], [194]. The time required to stand from a sitting position can uncover this type of impairment [195]. Sit-to-stand (STS) duration has been found to be significantly longer for those with cognitive impairments compared to cognitively healthy individuals [196]. Bed exits provide a chance to measure the duration of this task at least once a day using an ambient monitoring system. STS tests with a sensorized bed have been demonstrated with older adults [133].

Patients who have moved from MCI to dementia would also benefit from ongoing screening of sleep disturbances. Sleep disturbances both exacerbate cognition difficulties [197], [198] and extend negatively to the caregivers of older adults with cognitive impairments [194]. The gold standard for diagnosis of sleep disorders is polysomnography with EEG. However, this is not appropriate in older patients with dementia [199-201]. The current practice for patients with cognitive impairment is to use wrist-worn actigraphy to derive sleep patterns [202], with caregiver reports playing a complementary role [203] [204]. However, sensors requiring no compliance or user maintenance are perhaps more suited to cognitive impairment.

\subsubsection{Monitoring in Palliative Care}

As the population ages, medical services will face a surge of patients dying or facing terminal diseases. Palliative care needs are growing consequently. In this area, patients are increasingly showing a preference for being cared for at home [205] and dying with dignity at home [206-208].

Up to this point, physiological monitoring in palliative care has been necessarily 
unsophisticated, where the use of conventional obtrusive sensors precludes the main goal of patient quality of life and comfort. By introducing non-contact sensing, insights into the last days of life could be revealed. Some breathing patterns that would be revealed represent normal end-of-life phenomena. However, prompt identification of breathing patterns that represent addressable pathology could improve symptom control and comfort [209-211]. A variety of breathing patterns are regularly observed at the end of life. These include: apnea (flow cessation), hypopneas (flow reduction), hyperpnea (deep breathing), tachypnea (rapid breathing), periodic breathing (crescendo and decrescendo patterns of breathing depth), and Cheyne-Stokes respiration (crescendo and decrescendo of breathing depth, followed by an apnea).

The ability to predict short survival time could be used to start the process of discharge to home earlier, before transferring the patient becomes too difficult. High respiratory rates, as well as breathing irregularity and breathing pattern changes are partial indicators for the last days of life [212], [213]. Periodic breathing is correlated with mortality in chronic heart failure patients [214]. Weight loss is another possible estimator for survival time [212], [215]. All of these parameters are measurable with bed-based ambient sensors.

While palliative care lends itself well to bed-based ambient sensing, there are repercussions to the signal processing required to reliably monitor breathing. Signal processing architecture must expect breathing irregularity. Furthermore, it must cope with additional movement from nursing care and the activities of daily living that are increasingly performed in bed as illness worsens.

Although foundational work has demonstrated the ability of ambient systems in laboratory settings, there are fewer studies with ambient systems observing participants in clinical care at home or in institutions. No such studies targeting palliative care have been done. The following two sections present studies of palliative care. 


\subsection{Experiment \#1: Motion Monitoring in Pallia- tive Care}

To learn more about the activities that take place while in bed in palliative care, a study was run to both quantify and qualify the motion by palliative care patients.

\subsubsection{Methods}

Two patients in the palliative care ward at Elisabeth Bruyère Hospital, in Ottawa, ON, Canada, were monitored by a 50-element pressure sensor array beneath the bed. Simultaneously, a researcher at the hospital observed and noted their movements. The notes were used to annotate the data and to timestamp their bed occupancy status and movement. The patients were labeled "PalA" and "PalB". PalA was 81 years old and female, PalB was 55 years old and male, and both had lung cancer. 14 hours of recordings were collected for each participant. The spanned time from first to last recorded data was longer due to a couple of short non-recorded interludes for both participants and a full day of stoppage between recordings for PalB.

\section{Signal Processing}

To detect and classify movements, two detectors were implemented, a gross movement state machine and a limb movement detector. These are shown in Fig. 8.2. The gross movement state machine segmented the data according to one of three states:

$\{S$ :out-of-bed, position shift, rest $\}$ with two events and their complements: $\{E$ :shift detected, bed occupied\}. Since it is impossible to exit or enter a bed without shifting position, no transition was required between the rest and out-of-bed states. All states also have self-loops (not shown), which were the default action. Events were triggered by simple range, mean, and standard deviation operations on sliding windows of data. 


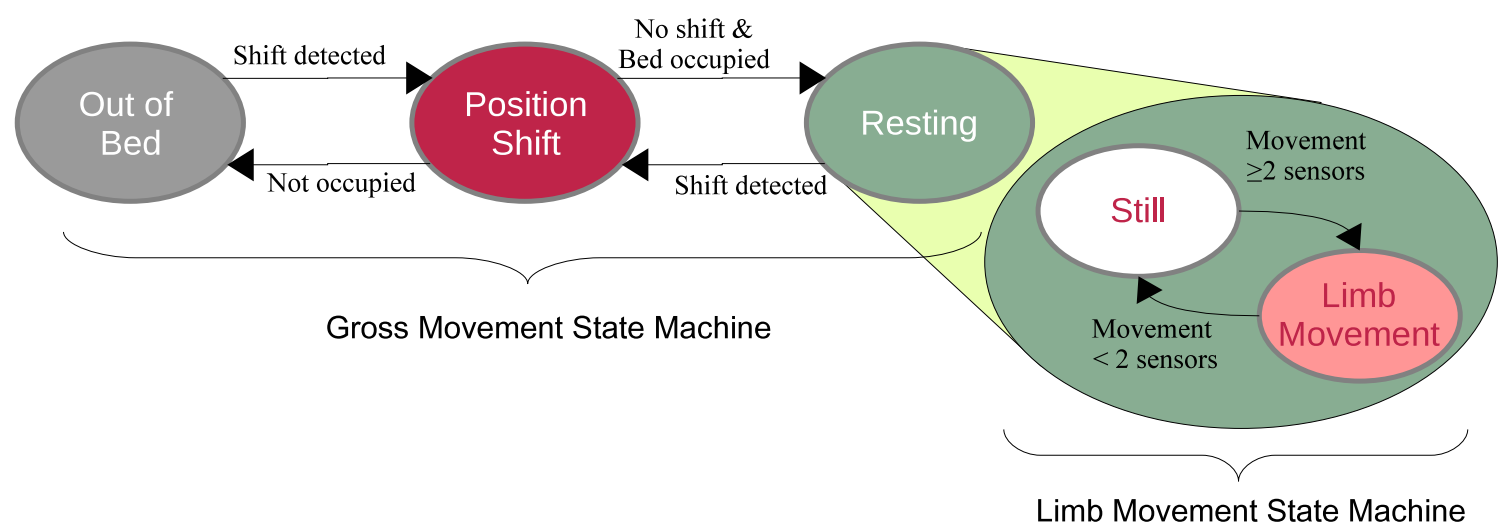

Figure 8.2: A finite state machine for segmenting data by bed occupant status

Substantial changes in pressure occur at multiple sensors during a position shift, so shifts were readily identified using the difference between the maximum and minimum value in the sliding window of length $L$. To catch many combinations of shift magnitudes and weight distributions, three thresholds on the range were used and a shift was declared if any of the thresholds were exceeded. The first compared the range at any sensor to a large threshold, $h_{\text {large }}$. The second required a minimum number of sensors $S_{\text {shift }}$ to surpass a medium threshold $h_{\text {med }}$. The last used a threshold $h_{\text {sum }}$ across the sum of all sensor ranges. To ensure this last one does not get tripped when strong breathing is present, the sensor range sum must also exceed six standard deviations above the mean range sum.

Occupancy was detected if $S_{\text {occup }}$ sensors were either loaded or active. Loading was declared once the mean in the sliding window exceeded $h_{\text {zero }}$; activity was declared when its standard deviation exceeded $h_{\text {act }}$. Since the zero values of sensors were not available and this value varies considerably between sensors, a best guess for $h_{\text {zero }}$ had to be made. $h_{\text {zero }}$ was set to the minimum of either $110 \%$ of the minimum value in the full data record or $20 \%$ of the maximum possible value. This second possibility ensures that a data record where a given sensor is never unloaded is not falsely detected as unloaded. 
Table 8.1: Threshold parameter settings

\begin{tabular}{|r||c|c|c|}
\hline Parameter & Maximum & Value & \% of Maximum \\
\hline \hline$h_{\text {med }}$ & 3072 & 400 & $10 \%$ \\
\hline$h_{\text {large }}$ & 3072 & 600 & $20 \%$ \\
\hline$h_{\text {sum }}$ & $3072 * 50$ & 2000 & $<1 \%$ \\
\hline$h_{\text {act }}$ & 3072 & 2 & $2 \%$ \\
\hline$S_{\text {shift }}$ & 50 & 3 & $6 \%$ \\
\hline$S_{\text {occup }}$ & 50 & 20 & $40 \%$ \\
\hline \hline
\end{tabular}

Parameter values and thresholds were set from histograms of data from preliminary benchtop testing with known position shifts and bed occupancies. The window length $L$ was set to 50 samples, representing five seconds of data sampled at $10 \mathrm{~Hz}$. Table 8.1 lists the selected thresholds, along with their maximum range and the percentage of this maximum that the threshold represents. Each of the 50 sensors had output ranges of $[0,3072]$, although most sensors never surpassed half of their maximum. Unloaded outputs were observed ranging between 105 and 465, depending on the sensor.

The resting segments include limb movements, which were smaller in amplitude to the position shifts and cannot necessarily be detected using preset thresholds without falsely identifying breathing as limb movement. Movement is instead identified statistically using a previously developed movement detection algorithm [124] which sets upper and lower control limits using the mean and standard deviation of a sliding data window with length $L=300$ (30 seconds). Two consecutive data points outside of the limits establish the beginning of movement. 


\section{Evaluation}

After the data was collected, annotated movement types were classified as either position shifts or limb movement. Some notes did not clearly define which of these classes occurred. For instance, the note "Fidgeting as she tries to get her drink from the side table" does not indicate whether a lean or shift was required to perform this action or if the side table is close enough that only a limb movement is required. In such cases, the assumption was made that a shift was required. Because it was not clear which nursing procedures would occasion movements or position shifts, nursing periods were excluded from analysis. Periods without annotations were also excluded, even if sensor data was available. For limb movement detection, periods with position shifts were also excluded since limb movements were not detected during position shifts.

Position shift and movement detection statistics were evaluated. True positives $(\mathrm{TP})$, false positives $(\mathrm{FP})$, true negatives $(\mathrm{TN})$, and false negatives $(\mathrm{FN})$ were incremented on an event-by-event basis according to annotated and detected events. Non-events were defined as the periods between annotated or detected events. The following statistics were compiled:

Accuracy \% (Acc.) Percentage of the events and non-events that were correctly detected as events or non-events, $T P+T N /(T P+F P+T N+F N)$;

Sensitivity \% (Sens.) Percentage of the total annotated events that were correctly detected as events, $T P /(T P+F N)$;

Specificity \% (Spec.) Percentage of the total annotated non-events that were detected as non-events $T N /(T N+F P)$;

Positive Predictive Value \% (PPV) Percentage of total detected events that were also annotated, $T P /(T P+F P)$; 
Negative Predictive Value \% (NPV) Percentage of non-detected events that were also not annotated events, $T N /(T N+F N)$.

The alternative to event-based evaluation is to evaluate sample by sample. However, annotations covered much longer periods than a given shift or movement actually lasted, and sample-based evaluation would skew results by introducing too many missed detections when there was in fact nothing to detect.

Shifts and movements were also evaluated for length and strength. The strength was measured as the ratio of the mean variance of the movement to the mean variance of the rest of the segment plus the mean variance of movement. A ratio near 1.0 indicates the motion is overwhelmingly stronger than rest, whereas a ratio near 0.5 indicates that the motion is roughly the same strength as rest.

\subsubsection{Results}

Fig 8.3 presents the major activities and percentage of time spent performing them, according to the annotations. PalA did not leave the bed and spent less time shifting position compared to PalB, although this may be more related to the style of annotation of the researchers. The researcher for PalB tended to group activities into a single longer duration annotation.

Fig 8.4 evaluates the percentage of time in activities that could be attributable to the participants' movement versus external intervention, such as nursing care. Some movements were considered joint, such as conversing with visitors or reaching for someone's hand. A good portion of movement was not performed by the participant, with at least $13 \%$ of movement time related to external intervention and $22 \%$ if joint activities were also counted. 

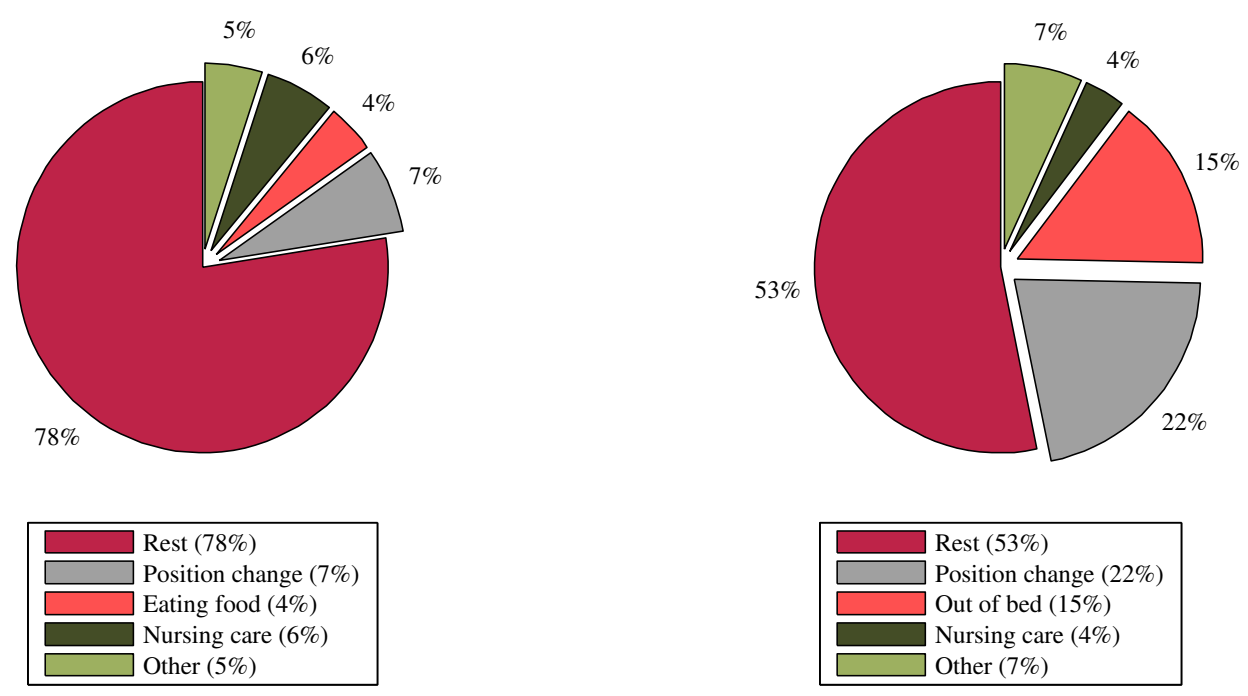

(a) PalA

(b) PalB

Figure 8.3: Pie chart of percentage of time spent performing activities according to annotations

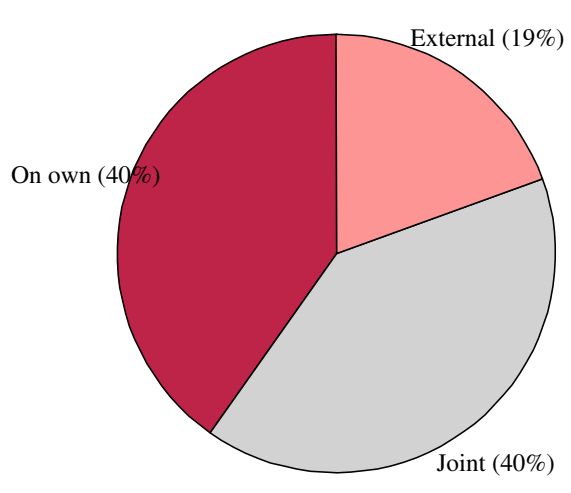

(a) PalA

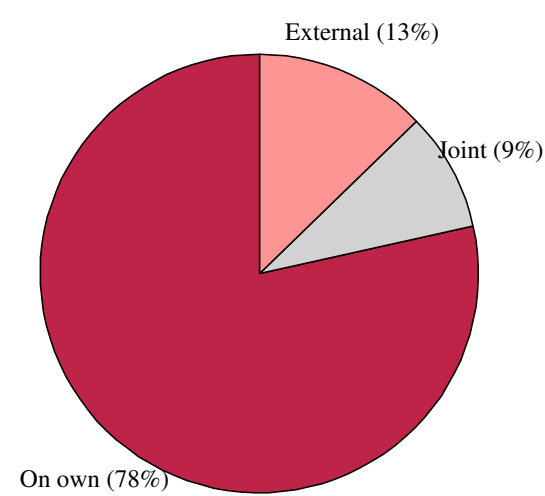

(b) PalB

Figure 8.4: Pie chart of the percentage of activities independently performed 
Table 8.2: Detection statistics for position shifts (\%)

\begin{tabular}{|r||c|c|c|c|c|}
\hline & Acc. & Sens. & Spec. & PPV & NPV \\
\hline \hline PalA & 78.0 & 63.0 & 83.6 & 58.6 & 85.9 \\
\hline PalB & 67.2 & 51.5 & 73.3 & 42.5 & 79.7 \\
\hline All & 72.1 & 56.7 & 78.0 & 49.3 & 82.7 \\
\hline \hline
\end{tabular}

\section{Bed Occupancy Detection}

Bed occupancy was detected and compared to annotations. All of the participants' time was spent in bed, except for three intervals by PalB. All three of these were detected and no false out-of-bed detections were made. Two short false in-bed detections were made during the last out-of-bed interval. During this interval, annotations indicate that the "sheets and linens were stripped and changed by nurse". The activity involved on the bed as well as the nurse potentially leaning on the bed to tuck the sheets in likely caused the bed occupancy detection.

\section{Detected Position Shifts}

Detected position shifts lasted an average of 6.7 seconds and resulted in an average movement strength ratio of 0.89 . Table 8.2 lists the resultant detection results for position shifts. There were both false detections that lowered the specificity and missed detections that lowered the sensitivity. In a number of cases, an annotation seemed to be late by a minute or two compared to the shift occurring in the sensor signals. Such cases caused both a false and a missed detection, which likely should have been true positives. In other cases, the assumption that a certain movement caused a position shift did not hold. For example, reaching for the glass of water did not cause a position shift on the sensors, so this became a false negative. Some detected shifts were clearly visible in the sensor signals but no annotation had been 
Table 8.3: Detection statistics for movement

\begin{tabular}{|r||c|c|c|c|c|}
\hline & Acc. & Sens. & Spec. & PPV & NPV \\
\hline \hline PalA & 53.8 & 100.0 & 52.9 & 3.7 & 100.0 \\
\hline PalB & 60.7 & 85.0 & 58.6 & 14.8 & 97.9 \\
\hline All & 55.4 & 91.4 & 54.2 & 6.2 & 99.5 \\
\hline \hline
\end{tabular}

made.

Table 8.2 may under-represent true accuracy and specificity. Since the detection statistics are event-based, long stretches of time without either detected or annotated position shifts only accounted for a single true negative event. By weighting the events by their duration, accuracy and specificity reach $93.8 \%$ and $99.8 \%$ respectively. However, sensitivity drops to $7.5 \%$ as false negatives durations were artificially long using annotated duration compared to the true positive durations lasting only the short detected duration.

\section{Limb Movement Detection}

Detected limb movements had an average duration of 3.4 seconds, and an average relative movement strength of 0.73 . Fig. 8.5 displays the detected movements with the annotated movements. The movement detector found many more movements than the observer. There are clusters of false negatives, particularly during the nights. The figure also shows the relative movement strength, showing that the nocturnal movements were smaller than the diurnal ones. Analysis of movement strengths showed the median non-annotated detections were significantly lower strength than the annotated ones. It may be that the sensors are more sensitive to movements than the observer, which might be more of a factor at night due to low light conditions and smaller movements.

Table 8.3 lists the resultant detection results for limb movements. As expected, 


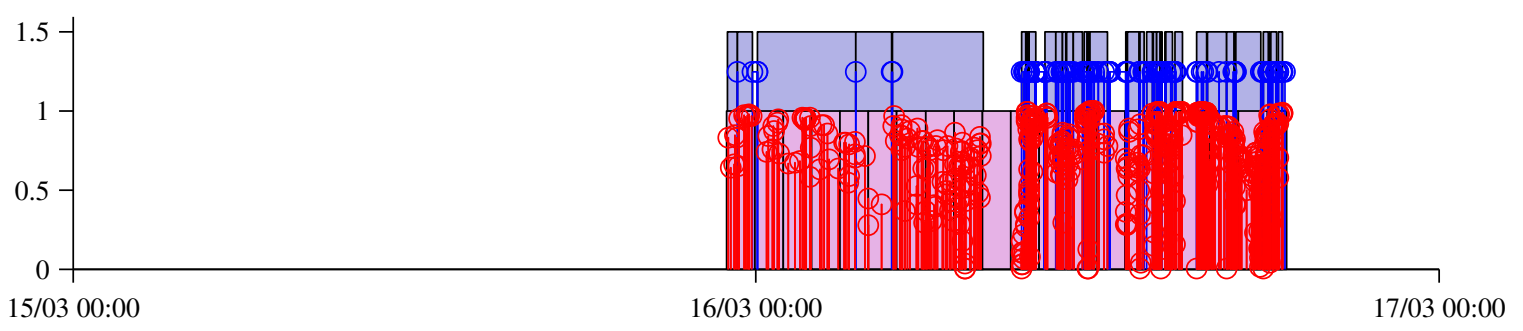

(a) PalA

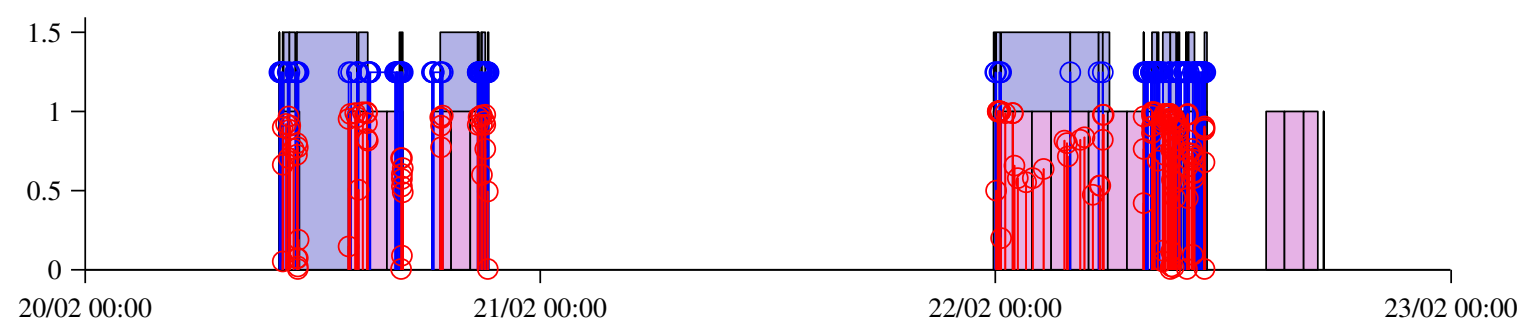

(b) PalB

Figure 8.5: Detected and annotated limb movements: A blue background indicates when annotation availability, and pink background blocks indicate recording intervals of the sensor data. Blue stems indicate annotated movements and red stems indicate detected movements. The height of the red stem indicates relative movement strength.

specificity is somewhat low, stemming potentially from the observer missing movement.

Two interesting patterns of movement were discovered and are shown in Fig. 8.6. Raising or lowering the bed caused a slow but steady pressure change visible on many sensors. Once seen, this pattern was observable numerous times. Sitting on the bed produced chaotic, but low amplitude signal changes. These small weight shifts represented neither limb movements nor breathing, but causes false limb movement detections and could cause difficulty during breathing analysis. 


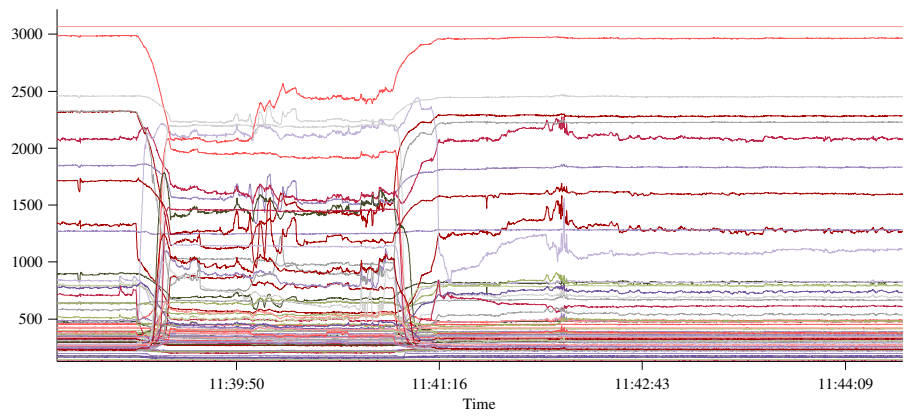

(a) Pressure sensor output of lowering/raising/sitting. Annotations for this segment were: "11:39: Top half of the bed is lowered. Legs are moved slightly to the left of bed by granddaughter. 11:40: Bed is moved back into sitting position."

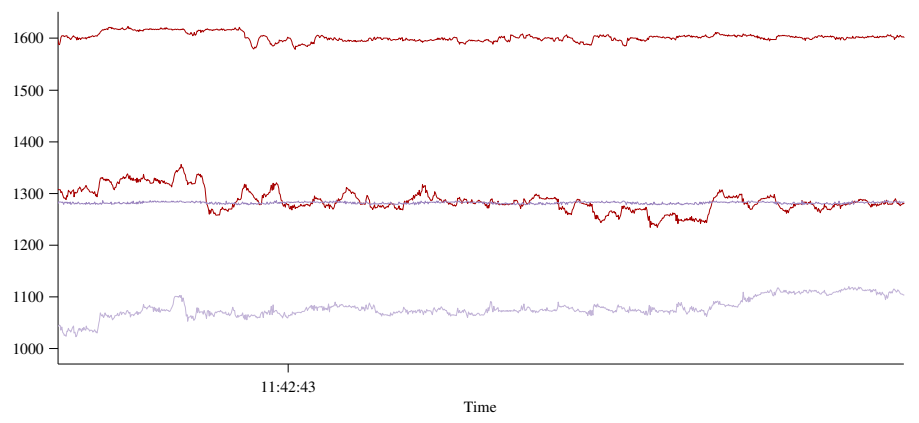

(b) Pressure sensor output while sitting (zoom-in of above plot after raising bed)

Figure 8.6: Two interesting pressure sensor patterns of movement discovered in the data

\subsubsection{Discussion}

Although the annotations did not necessarily allow precise comparisons, they do provide nonetheless a good balance between research requirements and ethical considerations. When interpreting results pertaining to movement, it is important to keep in mind the potential of a secondary movement instigator. For example, washing a patient involves both moving the patients' limbs and shifting the patient's position repeatedly, lasting more than ten minutes. Tracking nurses in a hospital has been accomplished by ambient sensors [216]. By adding such tracking to a system, movements could be more accurately assigned to the bed occupant.

The gross movement state machine, requiring only relatively simple operations, 
would be appropriate for real-time implementations. Even with its simplicity, initial testing has shown it to be effective.

Sitting is a difficult state to interpret due to the long period of disorder and plausible amplitudes for breathing. Attempting to extract breathing during sections of sitting would produce a chaotic signal that is difficult to automatically differentiate from very irregular breathing. A fourth state in the gross movement state machine may be necessary to differentiate sitting segments from resting segments.

\subsection{Experiment \#2: Long-term Monitoring in Palliative Care: A Pilot Study}

The feasibility of using ambient sensing in palliative care to detect respiratory disorder was tested with a healthy population at home and a population in palliative care at Elisabeth Bruyère Hospital. Due to the early stage of this research, as well as the sensitive nature and goals of palliative care, reference respiband monitoring was not performed. The use of obtrusive instruments, even for research, is discouraged in palliative care. Rather, differences between the two populations were exploited: it was hypothesized that a palliative care population would have more breathing irregularity than healthy individuals and that a diagnosis including lung impairment, such as lung cancer, would cause greater breathing irregularity than other diagnoses. To test these hypotheses, two parameters to quantify breathing irregularity were proposed. Also, extracted breathing rates were compared and qualitative observations of breathing were made.

To quantify breathing irregularity, extracted breathing signals were segmented into individual breaths. A signal processing architecture was devised to extract the breathing signal and segment it, considering the palliative care signal environment. 


\subsubsection{Methods}

\section{Equipment Setup}

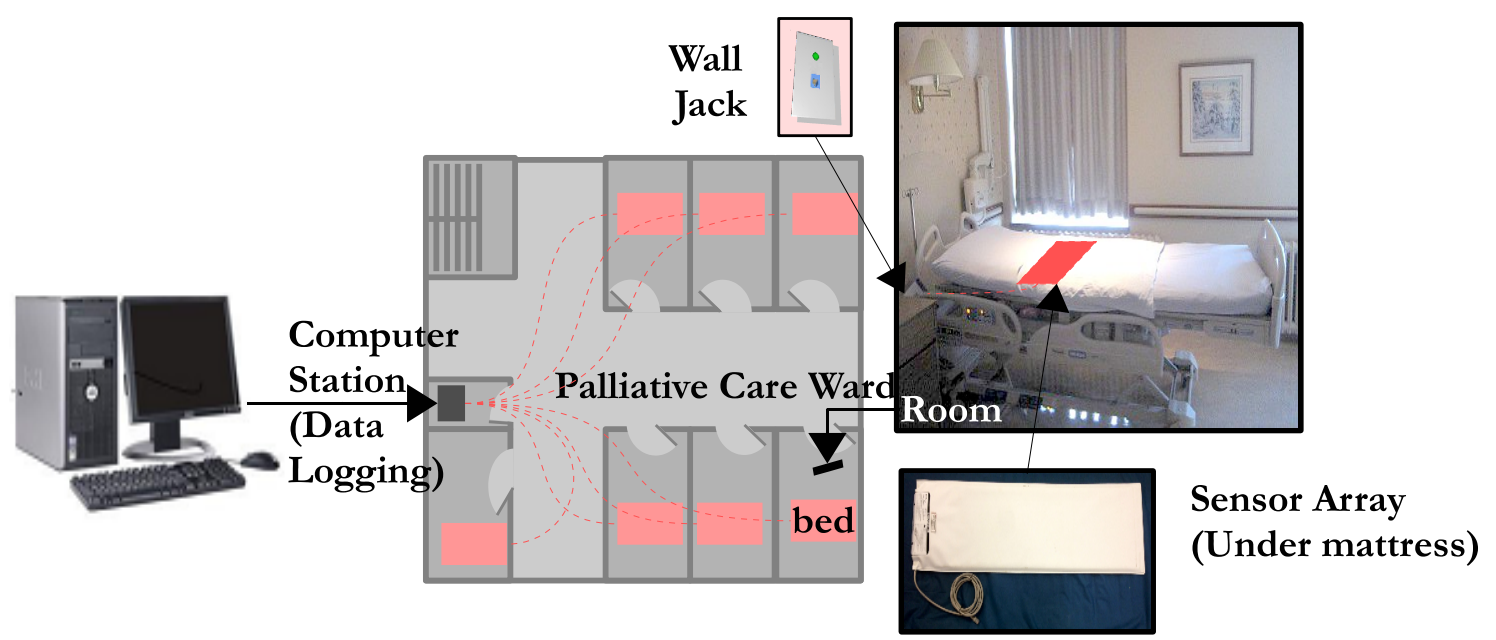

Figure 8.7: Diagram of the installation of sensor mats in the palliative care ward. Bedside wall jacks connected the mats to a main computer terminal.

Fig. 8.7 depicts the setup of sensor mats in the palliative care ward at Elisabeth Bruyère Hospital, Ottawa, ON. In 2007, twelve rooms underwent renovations which included installing cabling and wall plate interface boxes for connection to the S4 sensors pressure mats. The serial cabling from these was routed to a closet outfitted with a computer running the data acquisition software. The computer interfaced to all of the serial cabling through an external multi-port serial hub. The setup allowed a single mat (24 sensors) to be placed under the mattress in each of the rooms and the data from all of the mats could be collected by the single computer.

All bed frames were articulated hospital beds, but the complexity ranged from simple manually operated flat panels, to button-actuated contoured panels. The mattresses on these beds were institutional mattresses, designed for long term use. Fig. 8.8 shows a picture of the articulated frame on which the sensor mat is placed. To sense breathing motion, mats are ideally placed longitudinally down the centre of a bed to capture motion from the shoulders, chest, and abdomen. However, when an 


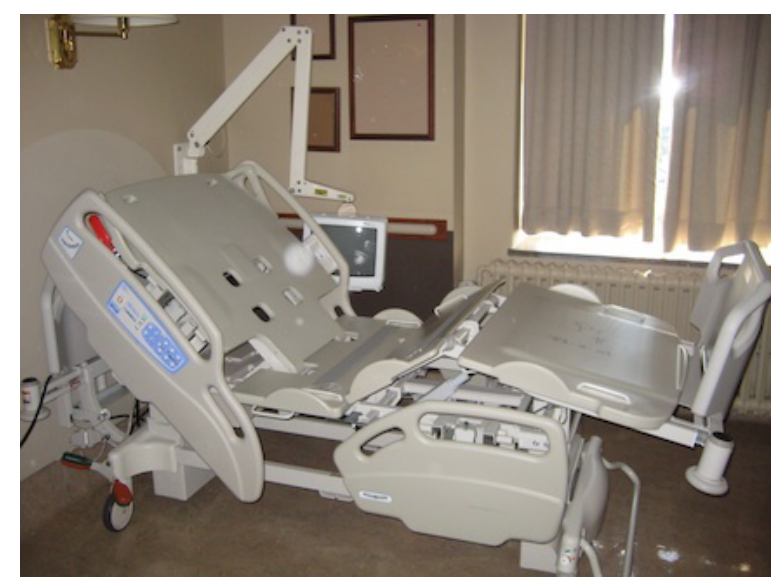

Figure 8.8: Photograph of actuated bed frame with contoured panels

articulated bed was raised, as shown in Fig. 8.8, the mat was bent by the articulation. Instead, a lateral placement was chosen to span the bed frame panels. This placement also ensured that the mat received motion from the sides of the bed.

It was also discovered that the mattress did not conform exactly to the bed while in the raised position. In that position, there were gaps between the mattress and the frame at the articulation points. However, the top row of sensors was still in contact with the mattress. It can be expected that fewer sensors are activated when a bed is in the sitting position.

\section{Data Collection}

Two sets of data were obtained from pressure sensors located below the mattress of participants. The first set was from data collected during the author's M.A.Sc. thesis [81]. The data from healthy participants were collected over a period of two to three nights while they slept in their own bed at home. The second set was from participants in the palliative care ward at Elisabeth Bruyère Hospital.

Six healthy participants formed the healthy set and 24 participants were recruited from palliative care. Data collection with palliative care participants was handled by staff at Elisabeth Bruyère Hospital. Sensor outputs were recorded continuously 
Table 8.4: Summary of participants for long-term monitoring in palliative care

\begin{tabular}{|c|c|c|c|c|c|c|c|c|}
\hline \multirow[b]{2}{*}{ Diagnosis } & \multirow[b]{2}{*}{ IDs } & \multicolumn{3}{|c|}{ Sex } & \multicolumn{2}{|c|}{$\begin{array}{c}\text { Lung } \\
\text { Involved? }\end{array}$} & \multicolumn{2}{|c|}{$\begin{array}{c}\text { Brain } \\
\text { Involved? }\end{array}$} \\
\hline & & $\mathbf{F}$ & M & $\mathbf{N} / \mathbf{A}$ & $\mathrm{Y}$ & $\mathbf{N}$ & $\mathrm{Y}$ & $\mathbf{N}$ \\
\hline Healthy & H1-H6 & 3 & 3 & 0 & 0 & 6 & 0 & 6 \\
\hline Gastro-Intestinal & GI1-GI8 & 3 & 4 & 1 & 5 & 3 & 1 & 7 \\
\hline Genito-Urinary & GU1-GU4 & 2 & 2 & 0 & 1 & 3 & 0 & 4 \\
\hline Lung & L1-L7 & 5 & 2 & 0 & 7 & 0 & 3 & 4 \\
\hline Other & O1-O5 & 4 & 1 & 0 & 2 & 3 & 1 & 4 \\
\hline Total & 30 & 17 & 12 & 1 & 15 & 15 & 5 & 25 \\
\hline
\end{tabular}

until the patient withdrew from the study, went home, or died. Room changes and transfers to acute care can occur; data collection was halted while the participant was not in a sensorized bed.

A chart audit was performed after data collection to gather pertinent information, including presence of lung and/or brain involvement, admission/readmission/discharge date and times, transfers out of the palliative care ward, and time of death. The presence of lung tumours was noted, since a diagnosis other than lung cancer does not preclude lung involvement. Metastatic cancer is prevalent in patients in the palliative care ward, and secondary lung tumors are common. Brain tumors may also have an effect on breathing, since brain centres that control breathing function could malfunction.

A resume of participants is profiled in Table 8.4. The number of females and males was representative of the typical Canadian elderly population, with more females than males (12 F, 9 M). Age, weight, and height were not available. Weight and height are not generally monitored in palliative care and disturbing the patients to take the measurements was not deemed necessary. 


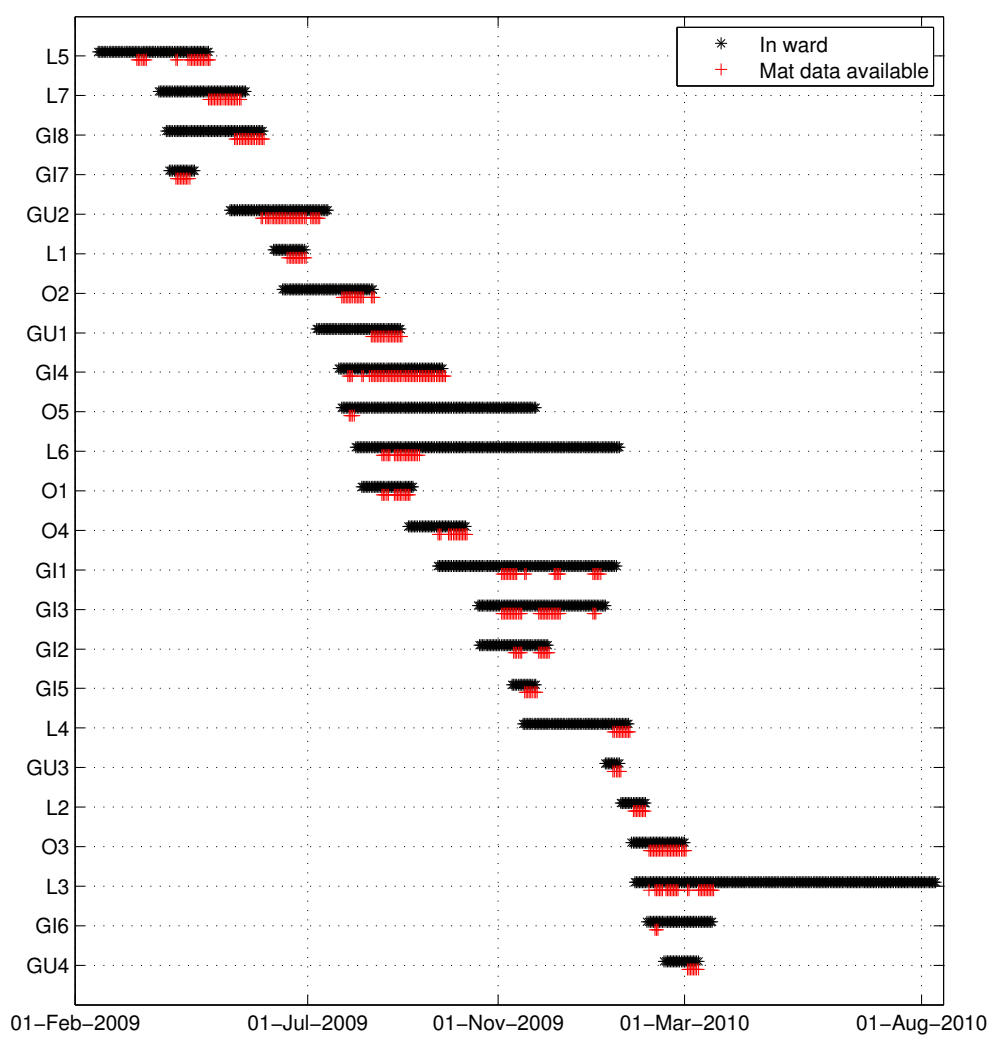

Figure 8.9: Collected Data from participants stay in palliative care (upper, black) and mat data availability (lower, red)

Fig. 8.9 shows the dates that the patient was in hospital and which dates were recorded by the pressure sensitive mat. In most cases, data was incomplete. Missing hours may be attributed to a number of factors. Firstly, participants may have changed rooms for a time period. Secondly, when a new participant was added, the system needed to be restarted. During the restart time, data could not be logged and restart times ranged from a few minutes to a quarter hour. Lastly, human error could occur, especially during the addition of new participants. Removal of current study participants from logging could (and did) occur occasionally at that point.

If data collection terminated early, the last few days of the patient's time in hospital were not recorded. Early termination could occur because of bed changes, transfers to acute care, participant withdrawal from study consent, or simply due to 
missing data files for the reasons listed in the previous paragraph. There were 13 deaths that occurred during data collection, one participant was discharged home alive, and ten participants had early data termination.

\section{Data Description}

There was no formalized protocol for the participants of this experiment to follow. Participants did not have their hospital stay disturbed.

The quality of the breathing signals recorded in the Palliative Care Ward was much lower than those collected in the controlled laboratory setting used in the previous chapters. Fewer sensors were available with breathing signals, breathing signals were generally of smaller amplitude, and some of the data segments between position changes did not have any sensors with available breathing. This may be attributable to the orientation of the pad, missed contacts between the mattress and the sensors when the bed is tilted up, and/or the weaker breathing of the participants.

\section{Breathing Signal Processing Architecture}

Signal processing architecture for palliative care patients must expect breathing irregularity and cope with extra movement interference, both from activities of daily living and from nursing care. The system must also deal with the participants' weaker breathing signals, and be resilient to low signal availability since there were fewer sensors picking up the breathing signal.

Preliminary tests of statistical change movement detectors with palliative care data revealed that these methods were limited by the irregularity of the breathing, particularly when apneas were involved. The end of an apnea would usually trigger the movement detection algorithm. Cheyne-Stokes respiration (CSR) is a breathing pattern that is common in palliative care and includes apneas every few breaths. 


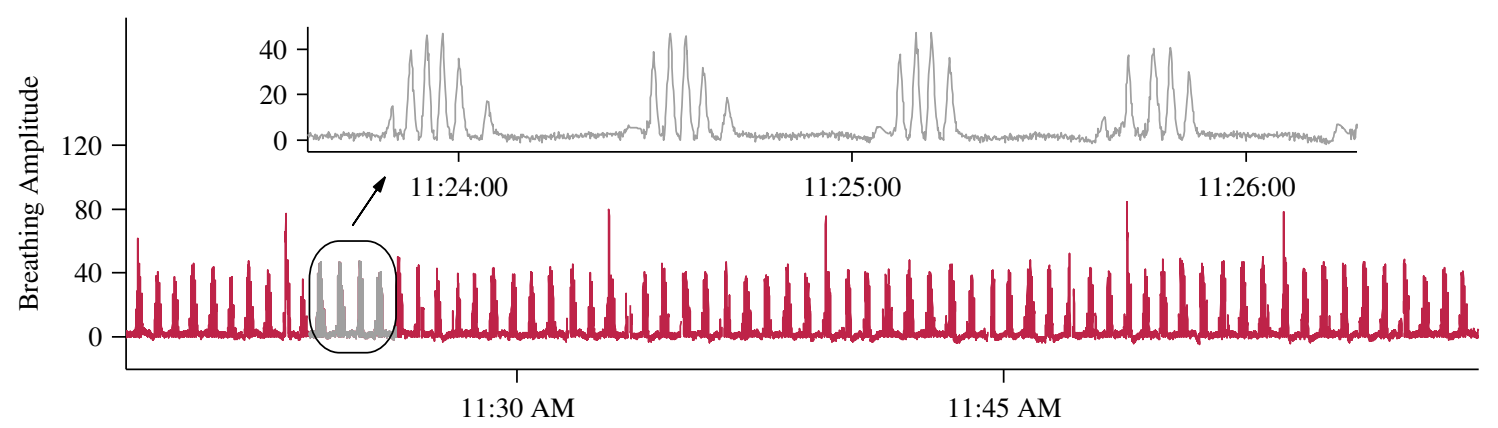

Figure 8.10: An example of Cheyne-Stokes respiration from a participant with brain cancer

Additionally, apneas in CSR manifest alongside changes in breathing depth; the amplitude changes can also trigger false movement detections. Fig. 8.10 depicts a CSR pattern, extracted from 45 minutes of the sensor data of participant O2, who had brain cancer. Almost all of the breaths in this segment were detected as movement. Conventional architecture of squelching breaths during detected movement would suppress the informative breathing patterns that the system is meant to quantify.

A new architecture was proposed to better discern movement and breathing, and is presented in Fig. 8.11. In this architecture, the gross movement state machine described in the previous study (Sec. 8.2.1) was used to squelch breathing output when not in bed or when large position shifts were occurring. Smaller limb movements and breathing were passed through the breathing fusion block. Breathing segmentation was performed on the fused signal and was used to remove drift and provide information to a fine segmentation block. This block also received input from a movement detector and a breathing quality signal to distinguish movements from false alarms. For this architecture, the fusion block in particular had to be robust to movement interference.

Breathing signal fusion was performed using the Adapt RD method outlined in Chapter 6. This method was shown to be resilient to a wide range of conditions, 


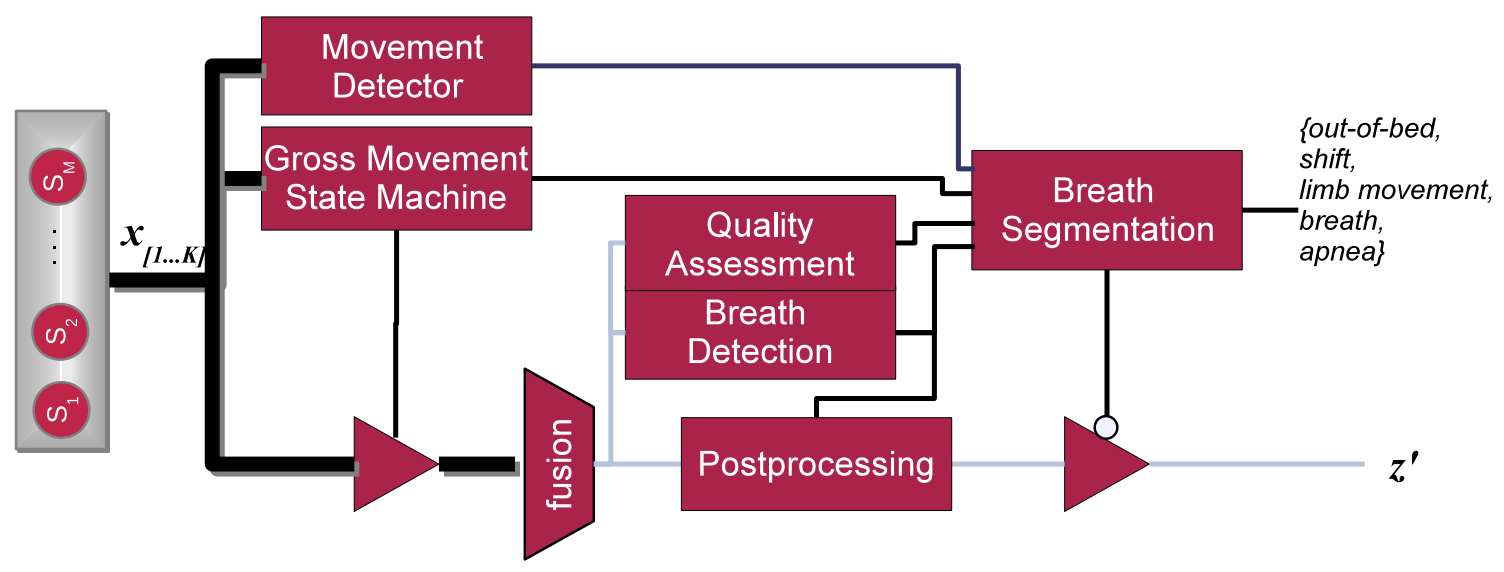

Figure 8.11: Proposed signal processing architecture

including movement and breathing irregularity without requiring a separate reversal detection stage. Individual breaths were detected using Cox Stuart trend analysis, as proposed by Moyles et al. [168] and developed further by Wilks et al. [152]. Cox Stuart trend analysis is a statistical tool that tests for an upward or downward trend in a data set by comparing sample values at the beginning of a data segment to those at the end [167]. The trend analysis provides a p-value for the hypothesis of the presence of an upward trend and one for the hypothesis of the presence of a downward trend. Trend tests were performed on sliding windows of data to find the beginning and end of upward and downward trends, using a threshold on the p-value of 0.9. The method provides for a refractory period between breaths [152] and requires that upward and downward trends alternate [168]. Breath peaks were located from the maximum value between the upward and downward trends; troughs were located as the minimum value between the previous downward trend and the current upward trend.

Apneas were suspected when an upward trend started more than ten seconds after the last downward trend ended. They were confirmed by comparing the standard deviation of the breathing signal within one-second sliding windows to a threshold at $20 \%$ of the median peak amplitude. Only segments not exceeding the threshold for 
at least ten seconds were retained, per the definition of apnea [217]. Low variance is a feature of apnea [61]. While a model-based apnea detector that looks for sudden variance changes works well for central apnea detection [189], this would not necessarily work well with palliative care data, where CSR patterns create slow variance changes around apneas.

A second post-processing stage removed signal shifts due to drift or movement. To remove signal shifts, a signal envelope was created by linear interpolation from trough to trough (lower enveloped) and peak to peak (upper envelope). This lower envelope was subtracted from the signal to relocate all troughs to a zero baseline. Although this subtraction removed details about breaths that empty the lungs more or less than others, this information is currently indistinguishable from drift.

Quality assessment was done using a by-product of the Cox Stuart trend analysis. With the sliding windows, these two p-values of the trend hypothesis are available for every sample, assigned according to the centre of the sliding window. Quality was set as the maximum of the two hypotheses at every sample. Quality was used in conjunction with limb movement detection to finalize the detected movements. Detected movements that did not overlap any detected breaths were automatically accepted as movements. For movement detections that did overlap with detected breaths, only those that had a mean quality smaller than 0.8 during the duration of the detected movement were considered to be movements. While this did have the effect of including some short movements, it was much less likely to detect breaths as movements, particularly during CSR. Additional limb movements were detected where breathing signal segments lay above the upper signal envelope, i.e., peaks that were not detected as breaths. 


\section{Respiratory Disturbance Analysis}

To screen generally for respiratory disturbance, breathing rates and two new indicator indices were extracted on an epoch by epoch basis. The new indicator indices were based on both respiratory effort amplitude and interval variability to flag potential problems. Disordered breathing often appears in respiratory signals through changes to respiratory interval or amplitude.

One minute epochs were used for analysis, with each epoch overlapping the previous by $50 \%$. The overlap ensures that subsequent epochs do not miss changes occurring at an epoch transition. Epochs were segmented according to detected movement so that movement periods could be ignored. To filter out segments that contained invalid breathing signals, such as those involving only the small weight shifts of a sitting participant, a minimum quality threshold was introduced for non-apnea periods within the epoch. For each segment, amplitudes of the breath peaks were recorded as well as the duration of intervals between peaks and between troughs. Epochs that contained fewer than three usable breath intervals or fewer than twenty contiguous seconds without movement artifact were discarded.

Two parameters were extracted from each valid epoch: the normalized standard deviation of the peak amplitudes $\left(\sigma_{\mathrm{RA}}\right)$ and the normalized standard deviation of the interval between peaks $\left(\sigma_{\mathrm{RI}}\right)$. To normalize for signal strength variations, respiratory peak amplitudes were divided by the mean of the amplitudes in each epoch. To normalize for natural rate differences, the respiratory intervals were divided by the epoch's mean interval. A new index labeled the Respiratory Amplitude and Interval Disturbance (RAID) index was proposed and calculated according to (8.1):

$$
\mathrm{RAID}=50\left((1-\alpha) \sigma_{\mathrm{RA}}+\alpha \sigma_{\mathrm{RI}}\right)
$$

Here, $\alpha$ was set to 0.5 to equally weight both parameters, although other weightings 
could be used. A second index, looks at the ratio between these two values, and is named the Sigma Ratio $\left(\sigma_{\text {ratio }}\right)$ :

$$
\sigma_{\text {ratio }}=\frac{\sigma_{\mathrm{RI}}}{\sigma_{\mathrm{RA}}}
$$

Data was summarized hourly, using the mean value in most cases, with the exception of using the maximum to discover the maximum breathing rate. For analysis of the impact of diagnosis on respiratory parameters, only data prior to a week before death were assessed. Later data could be impacted by the effects of approaching mortality. Furthermore, because the number of available days per participant was not equal, data was uniformly randomly sampled for a total of seven days of data per participant. Data from participants with fewer than seven days of recordings were sampled with replacement while data from the other participants were sampled without replacement. Data for analysis of mortality predictors comprised the recordings from the 13 participants with recordings at death. Analysis was from three weeks prior to mortality to the last day.

\subsubsection{Results}

More than a million epochs were analyzed of which $68 \%$ were considered viable, containing at least 20 contiguous seconds of breathing and at least three breathing intervals. Fig. 8.12 shows the percentage of valid epochs for a given quality threshold for the remaining epochs. The percentage of valid epochs was stable near $100 \%$ until the quality threshold neared 0.7. At that point, it dropped precipitously. Given that movements were already individually screened for quality, epochs that contained movement were more likely to have a slightly higher quality. A value of 0.75 was chosen as the validity threshold, discarding $13 \%$ of the viable epochs.

Many disordered breathing patterns were observed from the extracted respiratory 


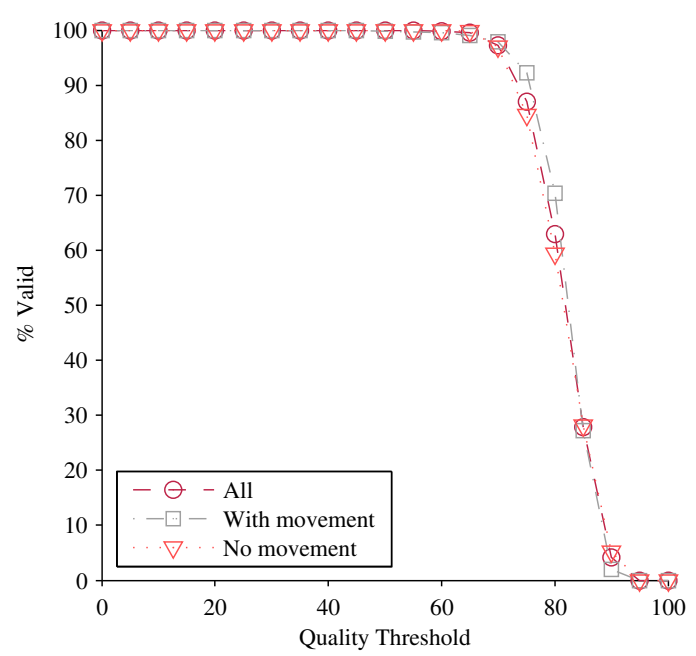

Figure 8.12: Percentage of valid epochs using a given quality threshold

signals of the palliative care participants. Fig. 8.13 displays examples of the first three minutes of breathing from the hours with the highest breathing rates, $\sigma_{\mathrm{RI}}$, and $\sigma_{\mathrm{RA}}$. All of the top 10 and 30 of the top 50 hourly RAID were ascribed to a single participant, O2. This participant had CSR for long stretches, and over many days.

\section{Respiration Parameter Hypotheses}

Fig. 8.14 displays a scatter plot of hourly averages of $\sigma_{\mathrm{RI}}$ and $\sigma_{\mathrm{RA}}$ pairs. The normalized interval and amplitude standard deviations tended to vary with each other. This tendency is somewhat intuitive since a deeper breath in the middle of other breaths manifests in both a longer breath and higher peak amplitude, and vice versa for a shorter breath.

The hypothesis that breathing patterns are more irregular for patients with cancer and particularly patients with lung and/or brain tumours is scrutinized in the boxplots of Fig. 8.15. Boxplots show boxes spanning the interquartile ranges, a mid line to denote the median, notches to show the confidence intervals around the median, and whiskers to show the full data range. Participants in palliative care tended to have slightly lower, but wider ranging, breathing rates. They also had much higher RAID 


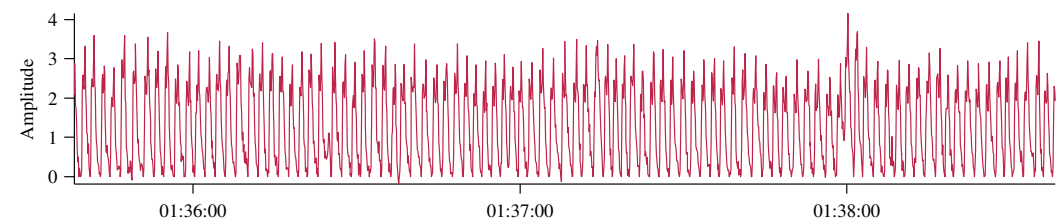

(a) High breathing rate (mean hourly $29.9 \mathrm{BPM}$ ) from $\mathrm{O} 6$

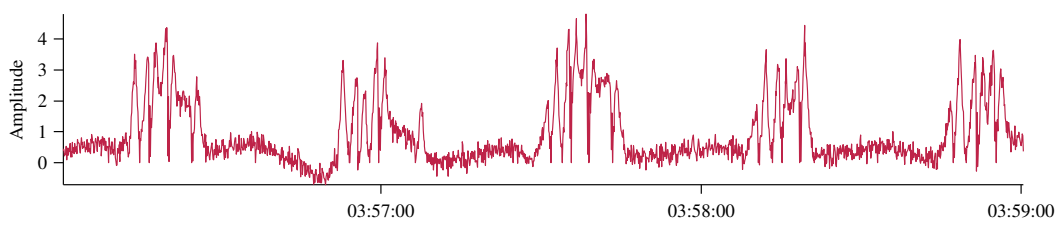

(b) High $\sigma_{\mathrm{RI}}$ (mean hourly $\sigma_{\mathrm{RI}}$ of 1.13) from L4

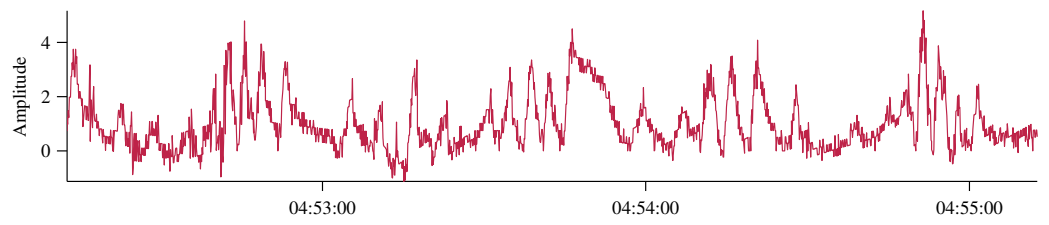

(c) High $\sigma_{\mathrm{RA}}$ (mean hourly $\sigma_{\mathrm{RA}}$ of 15.1 ) from L4

Figure 8.13: Examples of breathing patterns observed in the extracted respiratory signal of palliative care participant data

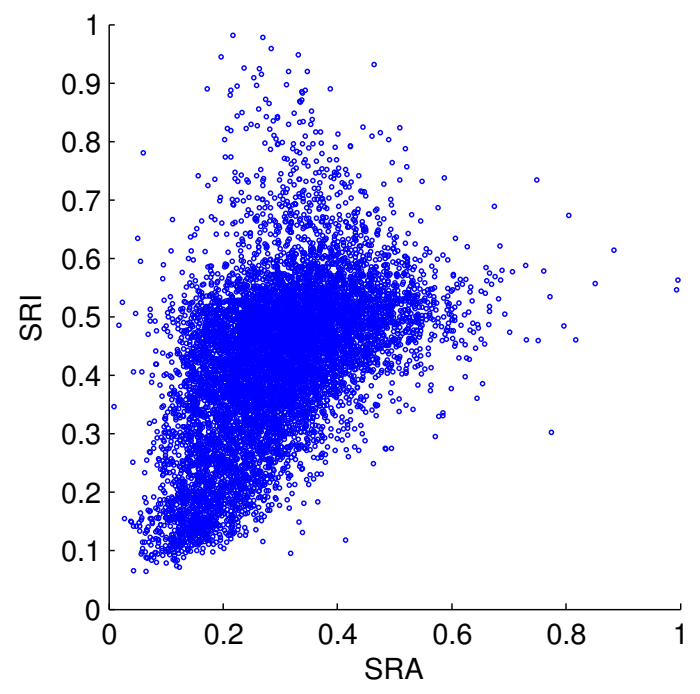

Figure 8.14: Scatter plot of hourly $\sigma_{\mathrm{RI}}$ and $\sigma_{\mathrm{RA}}$ 


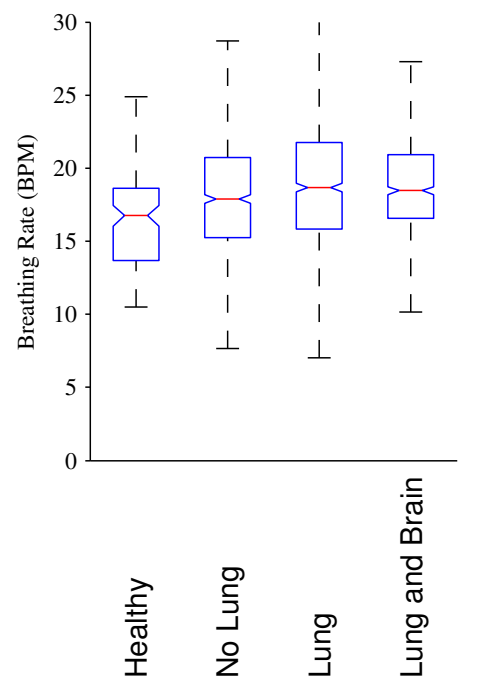

(a) Breathing rates

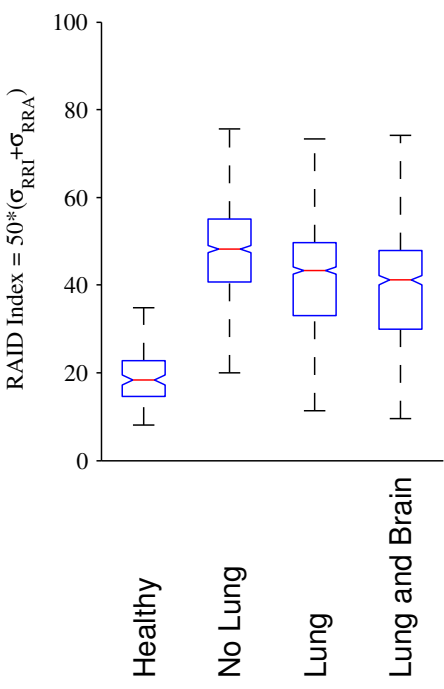

(b) RAID

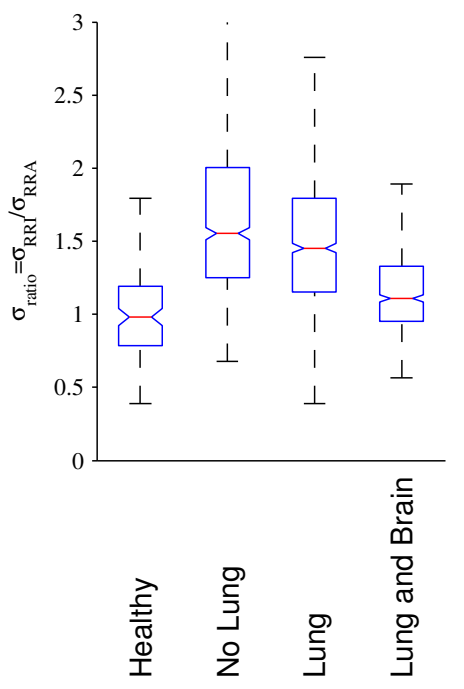

(c) $\sigma_{\text {ratio }}$

Figure 8.15: Boxplots of hourly respiratory parameters according to lung involvement

and $\sigma_{\text {ratio }}$ indices. Healthy participants had $\sigma_{\text {ratio }}$ indices close to unity, indicating that changes to breathing depth were directly related to changes in the time between breaths. The hypothesis that lung or brain involvement entrains more disorder was not supported by the results. In fact, the RAID and $\sigma_{\text {ratio }}$ each eased for the patients with these involvements.

\section{Predictors of Survival Time}

Fig. 8.16 displays the behaviour of the mean value of respiratory parameters as the participants approached mortality. For most of these parameters, a progression started between 10 and 15 days before death. Breathing rates and the prevalence of tachypnea $(B P M \geq 25)$ increased initially and then dropped. All 13 participants had tachypnea in the last week of life, although only eight had it in the last three days of life. The $\sigma_{\text {ratio }}$ index, displaying the most sensitivity to decreases in survival times, rose considerably near the end of life, influenced particularly by dropping $\sigma_{\mathrm{RA}}$. 


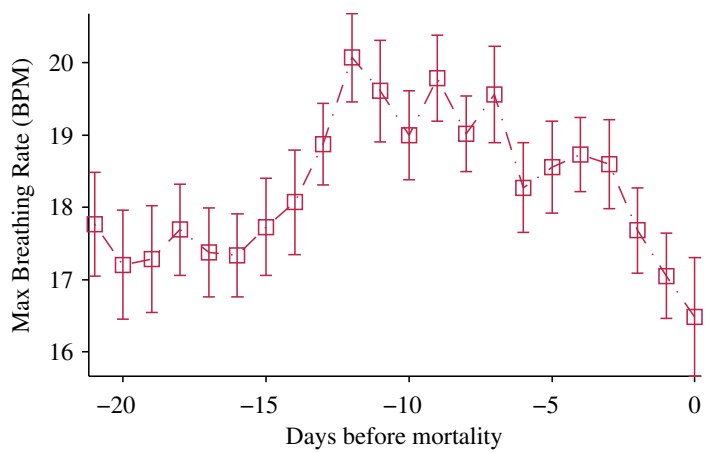

(a) Maximum Breathing rates

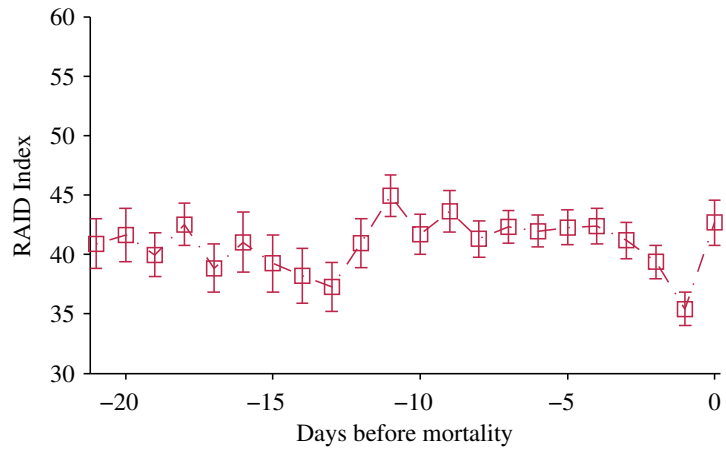

(c) RAID

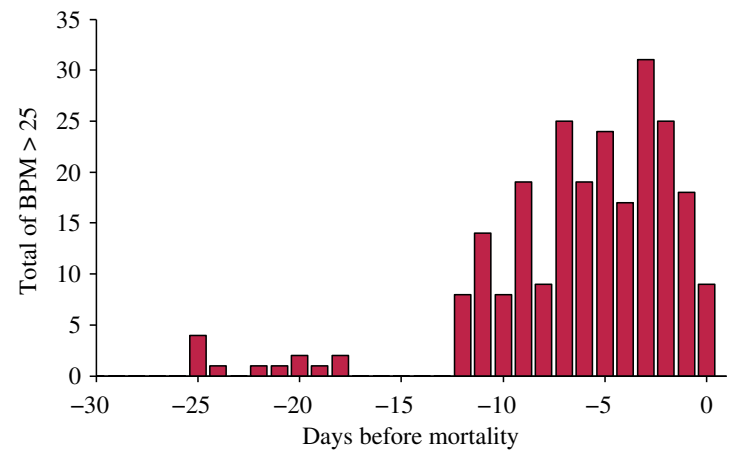

(b) Total number of hours of tachypnea

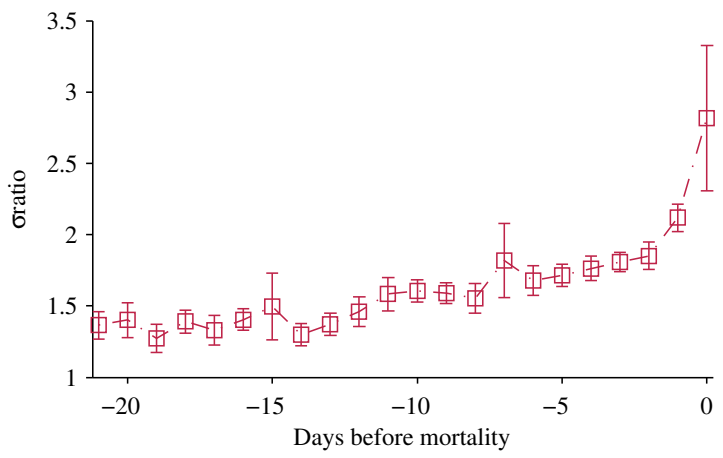

(d) $\sigma_{\text {ratio }}$

Figure 8.16: Mean respiratory parameters according to number of days from mortality 
In as much as breathing was more disturbed in the two weeks before death, the presence of most breathing patterns was not necessarily indicative of imminent mortality. Tachypnea was observed in three participants more than three weeks prior to death (O3,GI4, and L5), while maximum breathing rates decreased in the last week. The behaviour of the RAID index at the end of life also dipped before the last day, and this was a consistent pattern across a number of participants. Participant O2 had the highest RAID indexes due to CSR, but had much lower RAID indexes in the few days leading up to mortality. The CSR may have been related to her diagnosis of brain cancer. Manual examination of her breathing signals showed that CSR was indeed replaced by more regular breathing during the last days.

\subsubsection{Discussion}

This small pilot study provides some evidence for the feasibility of non-invasive, nonobtrusive monitoring of respiration in an inpatient and home-based palliative care population using bed-based pressure sensor arrays. The RAID and $\sigma_{\text {ratio }}$ indices clearly delineated between healthy participants and participants with cancer. The unexpected development that patients with lung and brain metastases had lower indices than those without remains to be explored.

The presence of tachypnea is a predictor of decreased survival time [212]. This was supported by all participants having tachypnea during their last week. A new breathing irregularity parameter, $\sigma_{\text {ratio }}$, also showed predictive value for survival time and further study is warranted. However, some participants displayed irregular breathing patterns many weeks before passing and in the days directly before mortality, some breathing patterns resolved. It is possible that combinations of these parameters would provide more accurate prediction, and future work could apply intelligent systems to this prediction. Other predictors of survivability include weight changes, time out of bed, and amount of movement [212]. These are not related to breathing 
and were not explored here, but could be extracted from a bed-based system.

The study was limited by the small sample size and lack of reference breathing sensors. Healthy participants were generally younger and it remains to be seen if age itself contributes to the breathing parameters.

Since movement of the patient restricts the intervals that are valid for breathing analysis, frequent movements could potentially mask breathing disorder. Intersubject and intra-subject nightly movement percentage in the course of overnight previous studies show high variability. Ranges of $10 \%$ to $30 \%$ and $3 \%$ to $32.5 \%$ were reported by Chen et al. [66] and Watanabe et al. [23], respectively. Kortelainen et al. discarded an average of $20 \%$ of the night due to movement or heart rate arrhythmia and noted that it "varied strongly between subjects" [43]. Aubert and Brauers reported ranges of discarded epochs from $9.5 \%$ for healthy participants up to $19 \%$ for participants with sleep disorders [27]. In this work, strong movements from nursing care and activities of daily living, combined with lower signal availability during resting segments resulted in a loss of more than one third of the epochs.

Not only could frequent movements mask breathing disorder, but they are also a potential source of error if they are erroneously detected as a breath, leading to high deviations in both $\sigma_{\mathrm{RI}}$ and $\sigma_{\mathrm{RA}}$. One mitigation strategy would be to recognize rest segments without any breathing available. In the previous study, for motion monitoring in palliative care (Sec. 8.2), sitting on the bed was identified as potentially problematic for breathing analysis. Automatic recognition of sitting segments could reduce the chance of falsely detecting breaths. Alternatively, rather than treating each epoch individually for quality analysis, assessing the quality of a full breathing segment could help exclude poor quality segments from analysis. Improving and validating the quality metric would also be useful. Kortelainen et al. used principal component analysis to differentiate movements from breathing since breaths consistently occur along the same principal axis, while the axis of movements differed [43]. 
The methodology presented here for breath segmentation could be extended to include this source of information by looking for out-of-the-ordinary changes to the Adapt RD weights in the quality assessment block. To improve breathing signals, using the context-aware fusion method developed in Chapter 7, may help alleviate conditions that weaken Adapt RD, such as reduced breathing signal availability.

In this work, prediction of survival time was examined. Another element that could be examined is detection of mortality. Initial testing on a limited number of participants showed this to be possible with a simple downward threshold on the variance, but expanding to more participants resulted in many missed detections. The noise at each sensor was too unique for a single threshold to be applied. Future work is to explore mortality detection with other salient features.

\subsection{Summary}

Pressure sensor arrays mark an exciting new technology for discreet point-of-care monitoring of patients with mild cognitive impairment (MCI), with full-blown cognitive impairment, or in palliative care. By sensorizing the bed, even the patients who are the most incapacitated can be monitored comfortably either at home or in institutional care. A rich set of assessment indicators can be formed from long-term, ambient sensor monitoring of nocturnal motor activity, sleep architecture, disordered breathing, and bed exits. These indicators could detect the progression of MCI to dementia, identify treatable conditions, and provide new insights into the last days of life.

Cognitive medicine and palliative care are particularly suited to the incorporation of ambient monitoring due to the sensitivity of their patients to more obtrusive technologies. Indeed, these patients are more likely to spend more time in bed than healthy individuals. Motion monitoring can quantify the number of position shifts 
and movements made on the bed. However, ascribing the motion to the patient rather than caregivers or visitors would require supplemental ambient sensors. Monitoring patient respiratory patterns can provide insights into physiological changes at the end of life and related survival times. Changes in breathing rate, interval, and amplitude are all indicative, but not specific to, the end of life. 


\section{Chapter 9}

\section{Conclusions}

Ambient sensing offers exciting new avenues of physiological monitoring and care, especially for populations that are not well suited to traditional laboratory monitoring or even wearable sensors. However, fusing physiological signals from multiple sensors located in an ambient environment presents various challenging conditions, stemming both from variable environmental response and from interference. Furthermore, the quasi-periodic and non-stationary nature of the physiological signals limits the use of spectral methods of signal analysis. Breathing signals are particularly prone to quasi-periodic and non-stationary behaviour and were the main application of this research.

\subsection{Summary of Research}

The fusion of a single breathing signal from multiple sensor inputs was evolved throughout this work. A single fused breathing signal is useful for breathing analysis, as it can be used for gauging both relative respiratory amplitude and the intervals between breaths. Variability of the amplitudes and intervals can be useful in clinical monitoring. 
Three aspects of signal-level fusion were considered. Firstly, the ambient multisensor environment was characterized. The feasibility of monitoring in ambient environments with unknown signal degradation was validated and the effect of cushioning was quantified. The non-linear behaviour of pressure sensors in a non-uniform array could pose problems for signal alignment using both reversals and delays. Signal alignment is required for many fusion methods. Using just reversal detection and not delay alignment, it was shown that poor reversal detection ultimately produced poor combining. Lastly, higher quality fused breathing signals were achieved through multisensor signal combining.

\subsubsection{Summary of Research Results: Chapter 4}

The experiments run in this chapter explored characterization of a sensor array and of signal loss in ambient environments. Some of the conditions that create variability in an ambient system were observed, including differentials in the response of sensors, unknown signal to noise power, and the availability of a given physiological signal at a given sensor. Both availability of breathing and breathing power diminished as a function of mattress size, although composition of the mattress and its density may play a role as well. When developing algorithms for physiological monitoring, it is important not only to test an algorithm in a laboratory environment, but also to make sure that it is robust to these real-life conditions. Sensor response can be affected by a number of non-linearities and non-uniformities, including the sensor material properties and the spatial response between sensors. While it is possible to calibrate a sensor array to linearize the response, calibration is a time consuming procedure. It was discovered that uncalibrated outputs could produce aligned signals that seemed unaligned by virtue of the sensor response. During signal alignment, delay measurements between sensors may be suspect. 


\subsubsection{Summary of Research Results: Chapter 5}

Correcting signal reversals is an important preparatory step in a number of fusion algorithms. Three strategies were proposed to detect reversals from ambient sensors: the correlation coefficient method (Corr.), decision directed slope reversal detection (DDSRD), and an adaptive version of DDSRD (Adapt RD). These all offered improvements over spectral phase analysis in conditions of interference and for nonstationary signals. Although the proposed correlation coefficient method improved reversal detection for non-stationary signals, it was hampered by interference. On the other hand, short-term trend analysis methods of DDSRD and Adapt RD showed resiliency to non-stationary behaviour and to interference. When compared to spectral phase analysis, these last two methods provided $9 \%$ more signal segments with acceptable signal qualities, resulting in $7 \%$ fewer segments with respiratory rate errors greater than 1 breaths per minute (BPM).

\subsubsection{Summary of Research Results: Chapter 6}

Three broad categories of ambient signal linear combining for breathing signal fusion were analyzed: sensor selection, diversity combining, and blind source separation. To this end, spectral estimations of signal and noise power were detailed and used in new fusion methods in all three broad categories. Eleven methods of linear combining were compared. The two techniques that performed best for fusion of real-life breathing signals and that were most resilient to simulated signal degradations were maximal ratio combining (MRC (PSD+EGC)) and Adapt RD. The latter was based on the adaptive method of reversal detection developed in the previous chapter. The two aforementioned methods provided an overall better estimate of the breathing signal. By applying Adapt RD, mean respiratory rate error fell another 5\%. However, in specific scenarios other algorithms did perform better. For instance, sensor selection 
and principal component analysis methods performed well when few sensor signals carried available breathing signals. Furthermore, these two methods did not require a separate reversal detection stage prior to fusion. Reversal detection can make or break the benefits derived from diversity combining algorithms. It was revealed that sensor selection performs better than diversity combining when reversal detection is poor.

\subsubsection{Summary of Research Results: Chapter 7}

An ensemble of fusion methods, or fusion agents, was assembled, selecting between each possible fusion agent given the conditions present in the input and output signals. Ranking and selecting fusion agents resulted in four agents chosen, with at least one from each of the general categories of agents presented in the previous chapter. The ensemble-based context selection method was able to more accurately fuse corrupted records than the top fusion agent, MRC (PSD+EGC), providing the best signal quality possible in over $50 \%$ of the test records, compared to just over $35 \%$ for MRC (PSD+EGC). For the multi-participant and multi-mattress experimental data, it was able to select the best algorithm $40 \%$ of the time, and nudged up signal quality and the number of records with less than $1 \mathrm{BPM}$ error for both. While the increase was minimal, the best pre-selected method for each set varied. With contextual selection, results were consistently good across all sets. When conditions are expected to be good, similar to laboratory conditions, it may not be worth the added complexity to implement an ensemble fuser. However, for medical applications and in scenarios of unknown signal conditions, it provides a more robust system. 


\subsubsection{Summary of Research Results: Chapter 8}

Pressure sensor arrays mark an exciting new technology for discreet point-of-care monitoring of patients with mild cognitive impairment (MCI), with full-blown cognitive impairment, or in palliative care. By sensorizing the bed, even the patients who are the most incapacitated can be monitored comfortably either at home or in institutional care. Indeed, these patients are more likely to spend more time in bed than healthy individuals. Motion monitoring can quantify the number of position shifts and movements made on the bed. However, ascribing the motion to the patient rather than caregivers or visitors would require supplemental ambient sensors. Monitoring patient respiratory patterns can provide insights into physiological changes at the end of life and related survival times. Changes in breathing rate, interval, and amplitude are all indicative, but not specific to, the end of life.

\subsection{Conclusions}

Continuous monitoring ambient medical monitoring could lead to faster interventions, when necessary, and less interference, where appropriate. Discreet and continual screening for specific disorders could also be performed, such as sleep disorders and respiratory disorders, as well as assessment of a general mortality risk from a combination of observed factors. It allows for the quasiperiodic signal behaviour that is a hallmark of many natural signals, particularly human physiological signals. It also holds paramount the mitigation of interference from sensors subject to noise, drift, and external interference. The objectives of this thesis were to:

1. Identify conditions of fallibility for fusion algorithms extracting physiological signals from ambient sensors;

2. Develop new, more robust multisensor fusion methods; 
3. Analyze new and existing algorithms, considering the identified conditions;

4. Identify new applications of clinical monitoring that would benefit from ambient sensing;

5. Apply ambient technology to the analysis of clinical data.

The first two objectives have gone hand-in-hand. By identifying conditions of fallibility that weaken fusion algorithms, new methods were developed that were more robust to the conditions encountered in ambient physiological monitoring. These new techniques for breathing signal extraction from ambient signals improved both breathing signal quality and respiratory rates derived from the breathing signal. By improving both reversal detection and fusion methods, respiratory rate errors were halved compared to equal gain combining with spectral phase reversal detection techniques.

One of the interesting facets of the developed algorithms is that they do not require knowledge of the posture of the bed occupant or position with respect to the sensors. In fact, the sensors themselves could be irregularly spaced, in unknown positions, and any number of sensors could be used. It has been shown that including more sensors increases the accuracy of the information, but there is no minimum number of sensors required for application of the methods proposed here.

Five reversal detection and 11 fusion algorithms were compared in conditions of noise and drift interference, movement artifact, reduced sensor availability and breathing period variability. It was revealed that some of these methods generally fared better than others. However, by determining the current conditions, a more appropriate algorithm can often be found for the current context, leading to improved and more robust signal extraction. 
In this thesis, fusion algorithms were evaluated for capability in the presence of movement. By selecting algorithms that performed well in its presence, the new architecture for breathing extraction presented in Chapter 8 was possible. The new architecture left limb movement detection until last, allowing false movement detections due to irregular breaths to be discounted.

Two new applications for clinical monitoring were identified that would benefit from ambient sensing, namely, cognitive medicine and palliative care. In both of these fields, the more sensitive needs of the patients preclude the use of traditional laboratory tests and obtrusive sensors. Two studies were conducted with participants from palliative care. Analysis of motion monitoring showed the feasibility of the ambient sensing in the palliative care environment, while breathing signal monitoring uncovered respiratory patterns near the end of life.

Not only can this system be used in institutional care, but it can also be brought into the home. By providing patients with palliative home care while still well enough to be transferred and improving the support facilities available at home, the wishes of these patients could be better fulfilled. The advances made in this thesis towards robust multisensor ambient signal processing move towards intelligent, ambient, and ubiquitous health monitoring in the comfort of home.

\subsection{Suggestions for Future Work}

There are a number of items for future work that reside outside the scope of this thesis.

- Non-linear combining fusion methods

In this work, linear combining was used to fuse signals at the data-level. Non-linear methods, such as wavelet analysis or empirical mode decomposition, may yield fusion 
performance improvements and should be considered. Strategies to select decomposition levels, while ignoring those without the breathing signal could also be helpful to reject interference.

\section{- Signal Quality Recognition}

The evaluation of signal quality under unknown ambient and signal conditions is an open research topic that was touched upon in this thesis, but was not fully developed. Recognition of breathing signal quality would be very useful both to reduce false breath detections and to improve selection methods. Spectrally estimated signal to noise ratio (SNR) was shown to be a good indicator of signal quality. However, under certain conditions, such as movement or breathing irregularity, spectrally estimated SNR did not correlate as well to actual signal quality. The gap between the results for the optimal sensor selection and the results from the proposed sensor selection algorithm could potentially be narrowed with a better estimate of signal quality. Research could be done to investigate new features or combinations of features that predict the optimal signal selection.

Signal quality could be used to determine whether a segment has a good enough breathing signal to be used for decision-making. A binary quantification as workable or non-workable would ensue for the segment. This evaluation could be performed by thresholding the signal quality or by a classifier, trained from known fusion signal respiband correlation.

- Classification of Movement Instigator

It was discovered that a notable portion of movements in palliative care are not executed by the patient themselves. Detecting the instigator of movement could allow the system to discern the motions related to care giving from those that indicate certain pathology, e.g. agitation due to pain. Another sensor set that detects motion in the room surrounding the bed could be used to infer if a given movement is 
instigated independently by the subject or is made by a caregiver. Alternatively, the data during periods of movement could be analyzed to see if any features could be used to directly detect external interference without any other sensors. For example, nursing care may causes certain weight shifts, such as side to side motion, that are not generally performed by a patient.

- Prediction of Survival Time

A number of interesting patterns were revealed when analyzing breathing signals from palliative care and the import of these patterns on the last days of life remains to be corroborated with larger clinical studies. Prediction of survival times using the respiratory parameters extracted in Chapter 8 , as well as movement, time out of bed, and weight changes could also be explored using intelligent systems.

- Movement Detection

Further work in movement detection and classification is warranted. It may be possible to modify the Adapt RD algorithm to provide movement detection as well as fusion weights. Movement detection was not a main goal for this thesis, but the outputs of movement detectors could be helpful in determining context for respiratory signal combining and improving the detection of individual breaths.

- Polarity Detection to Match Inhalation and Exhalation

The breathing signal analysis performed in this work did not consider parameters related to inhalation versus exhalation since breathing polarity was not well established. A rising signal could indicate either inhalation or exhalation. Kortelainen et al. performed this correction by observing that "inhalation shows a sharp peak in the signal in comparison with the longer exhalation valley" [43]. However, their methodology for this correction was not detailed. Further study and validation is 
required, particularly as the non-linearities explored in Chapter 4 could cause signal shapes with these peaks reversed simply because of sensor response.

- Detecting Thoracicabdominal Asynchrony

The methods proposed for detection of phase reversals in Chapter 5 could be tested for use with respibands for detection of thoracic asynchrony and compared to methods reviewed by Prisk et al. [161], and Motto et al. [104]. Phase reversal detection from non-ambient sensors such as respibands is not in the scope of this thesis, but could be useful for automated analysis of polysomnography data.

Detecting thoracicabdominal asynchrony that occurs while on a sensorized bed would also be of interest. The current methods of reversal detection assume that all reversals are systemic in nature rather than physiological. The behaviour of sensor data during episodes of asynchrony could be explored. 


\section{List of References}

[1] G. Acampora, D. Cook, P. Rashidi, and A. Vasilakos. "A survey on ambient intelligence in healthcare." Proc. IEEE 101(12), 2470-2494. ISSN 0018-9219 (2013).

[2] D. J. Cook, J. C. Augusto, and V. R. Jakkula. "Ambient intelligence: Technologies, applications, and opportunities." Pervasive and Mobile Comput. 5(4), 277-298. ISSN 1574-1192 (2009).

[3] C. Ramos, J. C. Augusto, and D. Shapiro. "Ambient intelligence: the next step for artificial intelligence." IEEE Intel. Syst. 23(2), 15-18. ISSN 1541-1672 (2008).

[4] A. Jain, A. Ross, and S. Prabhakar. "An introduction to biometric recognition." IEEE Trans. Circuits Syst. Video Technol. 14(1), 4-20. ISSN 1051-8215 (2004).

[5] T. Kleinberger, M. Becker, E. Ras, A. Holzinger, and P. Mller. "Ambient intelligence in assisted living: Enable elderly people to handle future interfaces." In C. Stephanidis, editor, "Universal Access in Human-Computer Interaction. Ambient Interaction," Number 4555 in Lecture Notes in Computer Science, pages 103-112. Springer Berlin Heidelberg. ISBN 978-3-540-73280-8, 978-3540-73281-5 (2007).

[6] R. C. Luo, C.-C. Yih, and K. L. Su. "Multisensor fusion and integration: approaches, applications, and future research directions." IEEE Sensors J. 2(2), 107-119 (2002).

[7] M. H. Jones, A. Arcelus, R. Goubran, and F. Knoefel. "A pressure sensitive home environment." In "Proc. 2006 IEEE Int. Workshop Haptic Audio Visual Environments and their Applications (HAVE 2006)," pages 10-14 (2006).

[8] M. Chan, D. Estve, C. Escriba, and E. Campo. "A review of smart homes present state and future challenges." Comput. Meth. Prog. Bio. 91(1), 55-81. ISSN 0169-2607 (2008). 
[9] J. A. Stankovic, Q. Cao, T. Doan, L. Fang, Z. He, R. Kiran, S. Lin, S. Son, R. Stoleru, and A. Wood. "Wireless sensor networks for in-home healthcare: Potential and challenges." In "Proc. High Confidence Medical Device Software and Systems (HCMDSS) Workshop," page 23 (2005).

[10] The New Oxford American Dictionary. "Robust." (2010).

[11] L. J. Epstein, D. Kristo, P. J. Strollo, N. Friedman, A. Malhotra, S. P. Patil, K. Ramar, R. Rogers, R. J. Schwab, E. M. Weaver, and M. D. Weinstein. "Clinical guideline for the evaluation, management and long-term care of obstructive sleep apnea in adults." J. Clin. Sleep Med. 5(3), 263-276. ISSN 1550-9389 (2009).

[12] C. Iber, S. Ancoli-Israel, J. Chesson, Andrew, and S. Quan. The AASM manual for the scoring of sleep and associated events: rules, terminology and technical specifications. American Academy of Sleep Medicine (2007).

[13] H. Schulz. "Rethinking sleep analysis." J. Clin. Sleep Med. 4(2), 99-103. ISSN 1550-9389 (2008).

[14] J. Alihanka, K. Vaahtoranta, and I. Saarikivi. "A new method for long-term monitoring of the ballistocardiogram, heart rate, and respiration." Am. J. Physiol. Regul. Integr. Comp. Physiol. 240(5), R384-392 (1981).

[15] Y. Nishida, M. Takeda, T. Mori, H. Mizoguchi, and T. Sato. "Monitoring patient respiration and posture using human symbiosis system." In "Proc. 1997 IEEE/RSJ Int. Conf. on Intelligent Robots and Systems (IROS '97), volume 2, pages 632-639 (1997).

[16] T. Hori, Y. Nishida, T. Suehiro, and S. Hirai. "SELF-Network: design and implementation of network for distributed embedded sensors." In "Proc. 2000 IEEE/RSJ Int. Conf. Intelligent Robots and Systems (IROS 2000)," volume 2, pages $1373-1378$ (2000).

[17] Y. Nishida and T. Hori. "Non-invasive and unrestrained monitoring of human respiratory system by sensorized environment." In "Proc. 2002 IEEE Sensors," volume 1, pages 705-710 (2002).

[18] T. Harada, T. Sato, and T. Mori. "Estimation of bed-ridden human's gross and slight movement based on pressure sensors distribution bed." In "Proc. 2002 IEEE Int. Conf. Robotics and Automation (ICRA '02)," volume 4, pages 3795-3800 (2002). 
[19] H. M. Van der Loos, N. Ullrich, and H. Kobayashi. "Development of sensate and robotic bed technologies for vital signs monitoring and sleep quality improvement." Auton. Robot 15(1), 67-79 (2003).

[20] H. Kimura, H. Kobayashi, K. Kawabata, and H. F. V. d. Loos. "Development of an unobtrusive vital signs detection system using conductive fiber sensors." In "Proc. 2004 IEEE/RSJ Int. Conf. Intelligent Robots and Systems (IROS 2004)," volume 1, pages 307-312 (2004).

[21] T. Harada, A. Sakata, T. Mori, and T. Sato. "Sensor pillow system: monitoring respiration and body movement in sleep." In "Proc. 2000 IEEE/RSJ Int. Conf. Intelligent Robots and Systems (IROS 2000)," volume 1, pages 351-356 (2000).

[22] J. H. Shin, Y. J. Chee, D.-U. Jeong, and K. S. Park. "Nonconstrained sleep monitoring system and algorithms using air-mattress with balancing tube method." IEEE Trans. Inf. Technol. Biomed. 14(1), 147 -156. ISSN 1089-7771 (2010).

[23] K. Watanabe, H. Ando, T. Ishikawa, and K. Kobayashi. "Noninvasive measurement of heartbeat, respiration, snoring and body movements of a subject in bed via a pneumatic method." IEEE Trans. Biomed. Eng. 52(12), 2100-2107. ISSN 0018-9294 (2005).

[24] D. Heise and M. Skubic. "Monitoring pulse and respiration with a non-invasive hydraulic bed sensor." In "Proc. 2010 Ann. Int. Conf. IEEE Engineering in Medicine and Biology Society (EMBC 2010)," pages 2119-2123 (2010).

[25] S. Sprager and D. Zazula. "Heartbeat and respiration detection from optical interferometric signals by using a multimethod approach." IEEE Trans. Biomed. Eng. 59(10), 2922-2929. ISSN 0018-9294 (2012).

[26] A. Ueno and Y. Yama. "Unconstrained monitoring of ECG and respiratory variation in infants with underwear during sleep using a bed-sheet electrode unit." In "Proc. 2008 Ann. Int. Conf. IEEE Engineering in Medicine and Biology Society (EMBC 2008)," pages 2329-2332. ISBN 1557-170X (2008).

[27] X. Aubert and A. Brauers. "Estimation of vital signs in bed from a single unobtrusive mechanical sensor: Algorithms and real-life evaluation." In "Proc. 2008 Ann. Int. Conf. IEEE Engineering in Medicine and Biology Society (EMBC 2008)," pages 4744-4747 (2008).

[28] W. B. SpillmanJr, M. Mayer, J. Bennett, J. Gong, K. E. Meissner, B. Davis, R. O. Claus, A. A. MuelenaerJr, and X. Xu. "A smart bed for non-intrusive 
monitoring of patient physiological factors." Meas. Sci. Technol. 15(8), 16141620 (2004).

[29] K. Watanabe, T. Tasaki, T. Nemoto, K. Yamakoshi, and W. Chen. "Development of biometry system in the sleep by pillow cuff installed on the occiput." Trans. Japanese Society Med. Biol. Eng 41(Suppl 1), 168 (2003).

[30] F. Wang, M. Tanaka, and S. Chonan. "Development of a PVDF piezopolymer sensor for unconstrained in-sleep cardiorespiratory monitoring." J. of Intell. Mater. Syst. and Struct. 14(3), 185 -190 (2003).

[31] T. Watanabe and K. Watanabe. "Noncontact method for sleep stage estimation." IEEE Trans. Biomed. Eng. 51(10), 1735-1748 (2004).

[32] W. Chen, X. Zhu, T. Nemoto, Y. Kanemitsu, K. Kitamura, and K. Yamakoshi. "Unconstrained detection of respiration rhythm and pulse rate with one underpillow sensor during sleep." Med. Biol. Eng. Comput. 43(2), 306-312 (2005).

[33] W. Chen, X. Zhu, and T. Nemoto. "A new sensory device and optimal position for monitoring HR/RR during sleep." In "Proc. 2009 World Congress on Medical Physics and Biomedical Engineering," volume 25/Vii Diagnostic and Therapeutic Instrumentation, Clinical Engineering, page 126. Munich, Germany (2009).

[34] Z. T. Beattie, T. L. Hayes, C. Guilleminault, and C. C. Hagen. "Accurate scoring of the apnea-hypopnea index using a simple non-contact breathing sensor." J. Sleep. Res. 22(3), 356-362. ISSN 1365-2869 (2013).

[35] S. Lokavee, T. Puntheeranurak, T. Kerdcharoen, N. Watthanwisuth, and A. Tuantranont. "Sensor pillow and bed sheet system: Unconstrained monitoring of respiration rate and posture movements during sleep." In "Proc. 2012 IEEE Int. Conf. Systems, Man, and Cybernetics (SMC 2012)," pages 1564-1568 (2012).

[36] S. Nukaya, T. Shino, Y. Kurihara, K. Watanabe, and H. Tanaka. "Noninvasive bed sensing of human biosignals via piezoceramic devices sandwiched between the floor and bed." IEEE Sensors J. 12(3), 431-438. ISSN 1530-437X (2012).

[37] D. Austin, Z. T. Beattie, T. Riley, A. M. Adami, C. C. Hagen, and T. L. Hayes. "Unobtrusive classification of sleep and wakefulness using load cells under the bed." In "Proc. 2012 Ann. Int. Conf. IEEE Engineering in Medicine and Biology Society (EMBC 2012)," pages 5254-5257 (2012). 
[38] A. M. Adami, M. Pavel, T. L. Hayes, and C. M. Singer. "Detection of movement in bed using unobtrusive load cell sensors." IEEE Trans. Inf. Technol. Biomed. 14(2), 481-490. ISSN 1089-7771 (2010).

[39] A. M. Adami, A. G. Adami, G. Schwarz, Z. T. Beattie, and T. L. Hayes. "A subject state detection approach to determine rest-activity patterns using load cells." In "Proc. 2010 Ann. Int. Conf. IEEE Engineering in Medicine and Biology Society (EMBC 2010)," pages 204-207 (2010).

[40] J. Kortelainen, M. Mendez, A. Bianchi, M. Matteucci, and S. Cerutti. "Sleep staging based on signals acquired through bed sensor." IEEE Trans. Inf. Technol. Biomed. 14(3), 776 -785. ISSN 1089-7771 (2010).

[41] A. M. Adami, T. L. Hayes, M. Pavel, and A. G. Adami. "Comparison of load cells and wrist-actigraphy for unobtrusive monitoring of sleep movements." In "Proc. 2009 Ann. Int. Conf. IEEE Engineering in Medicine and Biology Society (EMBC 2009)," pages 1314-1317. ISBN 1557-170X (2009).

[42] Z. Beattie, C. C. Hagen, M. Pavel, and T. Hayes. "Classification of breathing events using load cells under the bed." In "Proc. 2009 Ann. Int. Conf. IEEE Engineering in Medicine and Biology Society (EMBC 2009)," pages 3921-3924 (2009).

[43] J. Kortelainen, M. van Gils, and J. Parkka. "Multichannel bed pressure sensor for sleep monitoring." In "Proc. Computing in Cardiology (CinC 2012)," pages 313-316 (2012).

[44] J. J. Liu, W. Xu, M.-C. Huang, N. Alshurafa, M. Sarrafzadeh, N. Raut, and B. Yadegar. "Sleep posture analysis using a dense pressure sensitive bedsheet." Pervasive and Mobile Comput. 10(Part A), 34-50. ISSN 1574-1192. In press. (2013).

[45] M.-C. Huang, J. Liu, W. Xu, N. Alshurafa, X. Zhang, and M. Sarrafzadeh. "Using pressure map sequences for recognition of on bed rehabilitation exercises." IEEE Journal of Biomedical and Health Informatics 18(2), 411-418. ISSN 2168-2194 (2014).

[46] D. Townsend. Context-Aware Algorithms for Sleep Apnea Monitoring and Sensor Acceptance Using Unobtrusive Pressure Sensors Arrays. Ph.D., Carleton University (Canada), Canada (2012). 
[47] M. Holtzman, A. Arcelus, I. Veledar, R. Goubran, H. Sveistrup, and P. Guitard. "Force estimation with a non-uniform pressure sensor array." In "Proc. 2008 IEEE Instrumentation and Measurement Technology Conf. (IMTC 2008)," pages 1974-1979. ISBN 1091-5281 (2008).

[48] A. Arcelus, M. Holtzman, I. Veledar, R. Goubran, H. Sveistrup, and P. Guitard. "Contact location estimation from a nonlinear array of pressure sensors." In "Proc. 2008 IEEE Int. Conf. Instrumentation and Measurement Technology (IMTC 2008)," pages 1969-1973. ISBN 1091-5281 (2008).

[49] M. Holtzman, D. Townsend, R. Goubran, and F. Knoefel. "Validation of pressure sensors for physiological monitoring in home environments." In "Proc. 2010 IEEE Int. Workshop Medical Measurements and Applications (MeMeA 2010)," pages 38-42 (2010).

[50] A. Arcelus, M. Holtzman, R. Goubran, H. Sveistrup, P. Guitard, and F. Knoefel. "Analysis of commode grab bar usage for the monitoring of older adults in the smart home environment." In "Proc. 2009 Ann. Int. Conf. IEEE Engineering in Medicine and Biology Society (EMBC 2009)," pages 6155-6158 (2009).

[51] M. Holtzman, R. Goubran, and F. Knoefel. "Robust coherent sensor combining with decision directed reversal correction." IEEE Trans. Instrum. Meas. Accepted.

[52] M. Holtzman, A. Arcelus, R. Goubran, and F. Knoefel. "Breathing signal fusion in pressure sensor arrays." In "Proc. 2008 IEEE Int. Workshop Medical Measurements and Applications (MeMeA 2008)," pages 71-76 (2008).

[53] D. I. Townsend, M. Holtzman, R. Goubran, M. Frize, and F. Knoefel. "Measurement of torso movement with delay mapping using an unobtrusive pressuresensor array." IEEE Trans. Instrum. Meas. 60(5), 1751-1760. ISSN 0018-9456 (2011).

[54] M. Holtzman, R. Goubran, and F. Knoefel. "Maximal ratio combining for respiratory effort extraction from pressure sensor arrays." In "Proc. 2011 IEEE Int. Workshop Medical Measurements and Applications (MeMeA 2011)," pages 88-92. Bari, Italy. ISBN 978-1-4244-9336-4 (2011).

[55] M. Holtzman, R. A. Goubran, and F. Knoefel. "Breathing sensor selection during movement." In "Proc. 2011 Ann. Int. Conf. IEEE Engineering in Medicine and Biology Society (EMBC 2011)," pages 381-384. Boston, MA (2011). 
[56] M. Holtzman and F. Knoefel. "Ambient context in breathing signal fusion." In "Proc. 2014 IEEE Int. Workshop Medical Measurements and Applications (MeMeA 2014)," Accepted. Portugal (2014).

[57] M. Holtzman, R. Goubran, F. Knoefel, and J. Pereira. "Measurement of respiratory changes in palliative care." In "Proc. 2012 IEEE Instrumentation and Measurement Technology Conf. (IMTC 2012)," pages 1472 -1476 (2012).

[58] V. Joshi, M. Holtzman, A. Arcelus, R. Goubran, and F. Knoefel. "Highly survivable bed pressure mat remote patient monitoring system for mHealth." In "Proc. 2012 Ann. Int. Conf. IEEE Engineering in Medicine and Biology Society (EMBC 2012)," pages 268-271 (2012).

[59] D. Townsend, M. Holtzman, R. Goubran, M. Frize, and F. Knoefel. "Relative thresholding with under-mattress pressure sensors to detect central apnea." IEEE Trans. Instrum. Meas. 60(10), 3281-3289. ISSN 0018-9456 (2011).

[60] D. Townsend, M. Holtzman, R. Goubran, M. Frize, and F. Knoefel. "Effect of windowing on central apnea detection." In "Proc. 2010 IEEE Int. Workshop Medical Measurements and Applications (MeMeA 2010)," pages 117-120. Ottawa, ON (2010).

[61] D. I. Townsend, M. Holtzman, R. Goubran, M. Frize, and F. Knoefel. "Simulated central apnea detection using the pressure variance." In "Proc. 2009 Ann. Int. Conf. IEEE Engineering in Medicine and Biology Society (EMBC 2009)," pages 3917-3920 (2009).

[62] A. Arcelus, M. H. Jones, R. Goubran, and F. Knoefel. "Integration of smart home technologies in a health monitoring system for the elderly." In "Proc. 21st Int. Conf. Advanced Information Networking and Applications Workshops (AINAW 2007)," volume 2, pages 820-825. Niagara Falls, ON, Canada (2007).

[63] H. Yamaguchi, H. Nakajima, K. Taniguchi, S. Kobashi, K. Kondo, and Y. Hata. "Fuzzy detection system of behavior before getting out of bed by air pressure and ultrasonic sensors." In "Proc. 2007 IEEE Int. Conf. Granular Computing," page 114. IEEE Computer Society. ISBN 0-7695-3032-X (2007).

[64] P. Chow, G. Nagendra, J. Abisheganaden, and Y. T. Wang. "Respiratory monitoring using an air-mattress system." Physiol. Meas. 21(3), 345-354. ISSN 0967-3334 (2000). 
[65] V. Verhaert, B. Haex, T. De Wilde, D. Berckmans, M. Vandekerckhove, J. Verbraecken, and J. Sloten. "Unobtrusive assessment of motor patterns during sleep based on mattress indentation measurements." IEEE Trans. Inf. Technol. Biomed. 15(5), 787-794. ISSN 1089-7771 (2011).

[66] W. Chen, X. Zhu, T. Nemoto, K. Kitamura, K. Sugitani, and D. Wei. "Unconstrained monitoring of long-term heart and breath rates during sleep." Physiol. Meas. 29(2), 1 (2008).

[67] M. Uchida, S. Ding, W. Chen, T. Nemoto, and D. Wei. "An approach for extractions of pulse and respiration information from pulsatile pressure signals." In "Proc. 2003 EMBS Asian-Pacific Conf. Biomedical Engineering," pages 180$181(2003)$.

[68] E. Pino, A. Dorner de la Paz, P. Aqueveque, J. Chavez, and A. Moran. "Contact pressure monitoring device for sleep studies." In "Proc. 2013 Ann. Int. Conf. IEEE Engineering in Medicine and Biology Society (EMBC 2013)," pages 41604163 (2013).

[69] A. Hart, K. Tallevi, D. Wickland, R. Kearney, and J. Cafazzo. "A contact-free respiration monitor for smart bed and ambulatory monitoring applications." In "Proc. 2010 Ann. Int. Conf. IEEE Engineering in Medicine and Biology Society (EMBC 2010)," pages 927-930. ISBN 1557-170X (2010).

[70] D. Mack, D. Mack, J. Patrie, P. Suratt, R. Felder, and M. Alwan. "Development and preliminary validation of heart rate and breathing rate detection using a passive, ballistocardiography-based sleep monitoring system." IEEE Transactions on Information Technology in Biomedicine 13(1), 111-120. ISSN 1089-7771 (2009).

[71] A. A. P. Wai, K. Yuan-Wei, F. S. Fook, M. Jayachandran, J. Biswas, and J.J. Cabibihan. "Sleeping patterns observation for bedsores and bed-side falls prevention." In "Proc. 2009 Ann. Int. Conf. IEEE Engineering in Medicine and Biology Society (EMBC 2009)," pages 6087-6090 (2009).

[72] M. Brink, C. H. Mller, and C. Schierz. "Contact-free measurement of heart rate, respiration rate, and body movements during sleep." Behav. Res. Methods 38(3), 511-21. ISSN 1554-351X (2006).

[73] K. Niizeki, I. Nishidate, K. Uchida, and M. Kuwahara. "Unconstrained cardiorespiratory and body movement monitoring system for home care." Med. Biol. Eng. Comput. 43(6), 716-724 (2005). 
[74] Y. Nishida, T. Hori, T. Suehiro, and S. Hirai. "Monitoring of breath sound under daily environment by ceiling dome microphone." In "Proc. 2000 IEEE Int. Conf. Systems, Man, and Cybernetics (SMC 2000)," volume 3, pages 18221829. ISBN 1062-922X (2000).

[75] D. Ding, R. A. Cooper, P. F. Pasquina, and L. Fici-Pasquina. "Sensor technology for smart homes." Maturitas 69(2), 131-136. ISSN 1873-4111 (2011).

[76] G. Demiris, B. K. Hensel, M. Skubic, and M. Rantz. "Senior residents' perceived need of and preferences for "smart home" sensor technologies." Int. J. Technol. Assess. Health Care 24(1), 120-124. ISSN 0266-4623 (2008).

[77] K. L. Courtney. "Privacy and senior willingness to adopt smart home information technology in residential care facilities." Methods Inf. Med. 47(1), 76-81. ISSN 0026-1270 (2008).

[78] P. Dai, F. Chen, Z. Cao, M. Wang, J. Shen, and X. Wang. "An EMD based algorithm for non-contact physiological monitoring." J. Comput. Inform. Syst. 9(3), 1061-1069 (2013).

[79] J. L. Jacobs, P. Embree, M. Glei, S. Christensen, and P. K. Sullivan. "Characterization of a novel heart and respiratory rate sensor." In "Proc. 2004 Ann. Int. Conf. IEEE Engineering in Medicine and Biology Society (EMBC 2004)," volume 3, pages 2223-2226 (2004).

[80] X. Zhu, W. Chen, T. Nemoto, Y. Kanemitsu, K. I. Kitamura, K. I. Yamakoshi, and D. Wei. "Real-time monitoring of respiration rhythm and pulse rate during sleep." IEEE Trans. Biomed. Eng. 53(12 Part 1), 2553-2563 (2006).

[81] M. Howell Jones. Autonomous patient monitoring with a pressure sensor array. M.A.Sc., Carleton University, Ottawa, ON (2006).

[82] P. Kutilek, J. Hozman, and P. Smrcka. "Identification of patient's physiological movements on the bed by strain gauge sensors detect torsion of the elements of the bed." In "Proc. 2012 15th Int. Symp. Mechatronika," pages 1-6 (2012).

[83] J. C. Lai, Y. Xu, E. Gunawan, E. C. Chua, A. Maskooki, Y. L. Guan, K.-S. Low, C. B. Soh, and C.-L. Poh. "Wireless sensing of human respiratory parameters by low-power ultrawideband impulse radio radar." IEEE Trans. Instrum. Meas. 60(3), 928-938. ISSN 0018-9456 (2011). 
[84] C. Li, J. Ling, J. Li, and J. Lin. "Accurate doppler radar noncontact vital sign detection using the RELAX algorithm." IEEE Trans. Instrum. Meas. 59(3), 687-695. ISSN 0018-9456 (2010).

[85] V. Vasu, N. Fox, C. Heneghan, and S. Sezer. "Using the lomb periodogram for non-contact estimation of respiration rates." In "Proc. 2010 Ann. Int. Conf. IEEE Engineering in Medicine and Biology Society (EMBC 2010)," pages 24072410. ISBN 1557-170X (2010).

[86] C. Li, J. Cummings, J. Lam, E. Graves, and W. Wu. "Radar remote monitoring of vital signs." IEEE Microwave Magazine 10(1), 47-56. ISSN 1527-3342 (2009).

[87] A. Zaffaroni, P. de Chazal, C. Heneghan, P. Boyle, P. R. Mppm, and W. T. McNicholas. "SleepMinder: an innovative contact-free device for the estimation of the apnoea-hypopnoea index." In "Proc. 2009 Ann. Int. Conf. IEEE Engineering in Medicine and Biology Society (EMBC 2009)," pages 7091 -9094 (2009).

[88] O. Postolache, P. Girao, E. Pinheiro, R. Madeira, J. Pereira, J. Mendes, G. Postolache, and C. Moura. "Multi-usage of microwave doppler radar in pervasive healthcare systems for elderly." In "Proc. 2011 IEEE Instrumentation and Measurement Technology Conf. (I2MTC 2011)," pages 1-5. ISBN 1091-5281 (2011).

[89] K. Mukai, Y. Yonezawa, H. Ogawa, H. Maki, and W. M. Caldwell. "A remote monitor of bed patient cardiac vibration, respiration and movement." In "Proc. 2009 Ann. Int. Conf. IEEE Engineering in Medicine and Biology Society (EMBC 2009)," pages $5191-5194$ (2009).

[90] T. Hori, Y. Nishida, H. Aizawa, S. Murakami, and H. Mizoguchi. "Distributed sensor network for a home for the aged." In "Proc. 2004 IEEE Int. Conf. Systems, Man, and Cybernetics (SMC 2004)," volume 2, pages 1577-1582. ISBN 1062-922X (2004).

[91] M.-Z. Poh, D. J. McDuff, and R. W. Picard. "Non-contact, automated cardiac pulse measurements using video imaging and blind source separation." Opt. Express 18(10), 10762. ISSN 1094-4087 (2010).

[92] K. Nakajima, Y. Matsumoto, and T. Tamura. "Development of real-time image sequence analysis for evaluating posture change and respiratory rate of a subject in bed." Physiol. Meas. 22(3), N21. ISSN 0967-3334 (2001). 
[93] K. Nakajima, Y. Matsumoto, and T. Tamura. "A monitor for posture changes and respiration in bed using real time image sequence analysis." In "Proc. 2000 Ann. Int. Conf. IEEE Engineering in Medicine and Biology Society (EMBC 2000)," volume 1, pages 51-54 (2000).

[94] V. Hers, D. Corbugy, I. Joslet, P. Hermant, J. Demarteau, B. Delhougne, G. Vandermoten, and J. P. Hermanne. "New concept using passive infrared (PIR) technology for a contactless detection of breathing movement: a pilot study involving a cohort of 169 adult patients." J. Clin. Monitor. Comp. page 19 (2013).

[95] T. Hayes, T. Riley, M. Pavel, and J. Kaye. "Estimation of rest-activity patterns using motion sensors." In "Proc. 2010 Ann. Int. Conf. IEEE Engineering in Medicine and Biology Society (EMBC 2010)," pages 2147-2150 (2010).

[96] K. Nakajima, T. Tamura, L. Lu, H. Miike, S. Kasaoka, K. Nakashima, T. Maekawa, and T. Togawa. "Evaluation of bed rest using a bed temperature monitor after acute myocardial infarction." In "Proc. 1995 Ann. Int. Conf. IEEE Engineering in Medicine and Biology Society (EMBC 1995)," volume 1, pages 689-690 (1995).

[97] T. Tamura, S. Miyasako, T. Fujimoto, and T. Togawa. "Monitoring bed temperature in elderly in the home [ECG/body movements]." In "Proc. 1996 Ann. Int. Conf. IEEE Engineering in Medicine and Biology Society (EMBC 1996)," volume 1, pages 57-58 (1996).

[98] H. Maki, H. Ogawa, S. Tsukamoto, Y. Yonezawa, and W. Caldwell. "A system for monitoring cardiac vibration, respiration, and body movement in bed using an infrared." In "Proc. 2010 Ann. Int. Conf. IEEE Engineering in Medicine and Biology Society (EMBC 2010)," pages 5197-5200. ISBN 1557-170X (2010).

[99] R. Murthy, I. Pavlidis, and P. Tsiamyrtzis. "Touchless monitoring of breathing function." In "Proc. 2004 Ann. Int. Conf. IEEE Engineering in Medicine and Biology Society (IEMBS 2004)," volume 1, pages 1196-1199 (2004).

[100] Y. G. Lim, K. K. Kim, and K.-S. Park. "ECG recording on a bed during sleep without direct skin-contact." IEEE Trans. Biomed. Eng. 54(4), 718-725. ISSN 0018-9294 (2007).

[101] M. Ishijima. "Long-term cardiopulmonary monitoring in bed without subject awareness." In "Proc. 1996 Ann. Int. Conf. IEEE Engineering in Medicine and Biology Society (EMBC 1996)," volume 1, pages 65-66 (1996). 
[102] P. Bard. Medical physiology. The CV Mosby Company, St. Louis, USA, 10th ed edition (1956).

[103] L. Johnson. Essential Medical Physiology. Academic Press, Burlington, MA, USA. ISBN 9780080472706 (2003).

[104] A. Motto, H. Galiana, K. Brown, and R. Kearney. "Automated estimation of the phase between thoracic and abdominal movement signals." IEEE Trans. Biomed. Eng. 52(4), 614-621. ISSN 0018-9294 (2005).

[105] S. G. Mallat. "A theory for multiresolution signal decomposition: the wavelet representation." IEEE Trans. Pattern Anal. Mach. Intell. 11(7), 674-693 (1989).

[106] O. Postolache, P. S. Girao, G. Postolache, and M. Pereira. "Vital signs monitoring system based on EMFi sensors and wavelet analysis." In "Proc. 2007 IEEE Instrumentation and Measurement Technology Conf. (IMTC 2007)," pages 1-4. IEEE. ISBN 1-4244-0588-2 (2007).

[107] E. C. Pinheiro, O. A. Postolache, and P. S. Girao. "Automatic wavelet detrending benefits to the analysis of cardiac signals acquired in a moving wheelchair." In "Proc. 2010 Ann. Int. Conf. IEEE Engineering in Medicine and Biology Society (EMBC 2010)," pages 602-605. IEEE. ISBN 978-1-4244-4123-5 (2010).

[108] N. E. Huang. "The empirical mode decomposition and the hilbert spectrum for nonlinear and non-stationary time series analysis." Proceedings: Mathematical, Physical and Engineering Sciences 454(1971), 903-995 (1998).

[109] P. Flandrin, G. Rilling, and P. Goncalves. "Empirical mode decomposition as a filter bank." IEEE Signal Processing Lett. 11(2), 112-114 (2004).

[110] B. Wang, X. Wang, A. Ikeda, T. Nagamine, H. Shibasaki, T. Sugi, and M. Nakamura. "Automatic reference selection for quantitative EEG component interpretation: Cross spectrum analysis based on bipolar EEG." In F. L. Wang, H. Deng, Y. Gao, and J. Lei, editors, "Artificial Intelligence and Computational Intelligence," Number 6320 in Lecture Notes in Computer Science, pages 79-86. Springer Berlin Heidelberg (2010).

[111] P. Varady, S. Bongar, and Z. Benyo. "Detection of airway obstructions and sleep apnea by analyzing the phase relation of respiration movement signals." IEEE Trans. Instrum. Meas. 52(1), 2-6. ISSN 0018-9456 (2003). 
[112] B. Wang, X. Wang, A. Ikeda, T. Nagamine, H. Shibasaki, and M. Nakamura. "Automatic detection of the topographical distribution of EEG rhythms based on an iterative adjustment of the averaged reference potential." Artif. Life Robotics 16(2), 243-247. ISSN 1433-5298, 1614-7456 (2011).

[113] D. L. Hall and J. Llinas. "An introduction to multisensor data fusion." Proc. IEEE 85(1), 6-23 (1997).

[114] D. Brennan. "Linear diversity combining techniques." Proc. IEEE 91(2), 331356 (2003).

[115] D. Brennan. "Linear diversity combining techniques." Proc. IRE 47(6), 10751102. ISSN 0096-8390 (1959).

[116] T. Wartzek, S. Weyer, and S. Leonhardt. "A differential capacitive electrical field sensor array for contactless measurement of respiratory rate." Physiol. Meas. 32(10), 1575. ISSN 0967-3334 (2011).

[117] J.-F. Cardoso. "Blind signal separation: statistical principles." Proc. IEEE 86(10), 2009-2025. ISSN 0018-9219 (1998).

[118] A. Hyvrinen, J. Karhunen, and E. Oja. Independent component analysis. John Wiley and Sons. ISBN 9780471405405 (2001).

[119] A. Hyvarinen. "Fast and robust fixed-point algorithms for independent component analysis." IEEE Trans. Neur. Net. 10(3), 626-634. ISSN 1045-9227 (1999).

[120] H. Li, B. S. Manjunath, and S. K. Mitra. "Multi-sensor image fusion using the wavelet transform." In "Proc. IEEE Int. Conf. Image Processing (ICIP-94)," volume 1, pages 51-55 (1994).

[121] X. Jiang, L. Zhou, and Z. Gao. "Multispectral image fusion using wavelet transform." In "Proc. SPIE 2898, Electronic Imaging and Multimedia Systems," volume 2898, pages 35-42 (1996).

[122] Q. Liu, W. Hu, and X. Jiang. "Contrast-based image fusion in wavelet domain." In "Proc. Sixth World Congress on Intelligent Control and Automation (WCICA 2006)," volume 2, pages 9574-9577 (2006).

[123] L. J. Chipman, T. M. Orr, and L. N. Graham. "Wavelets and image fusion." In "Proc. SPIE's 1995 International Symposium on Optical Science, Engineering, and Instrumentation," volume 3, pages 248-251. International Society for Optics and Photonics (1995). 
[124] M. H. Jones, R. Goubran, and F. Knoefel. "Identifying movement onset times for a bed-based pressure sensor array." In "Proc. 2006 IEEE Int. Workshop Medical Measurements and Applications (MeMeA 2006)," pages 111-114 (2006).

[125] \{Statistics Canada\}. "Healthy aging indicators, by age group and sex, household population aged 45 and over, canada and provinces, occasional (CANSIM table 105-1200)." Technical report. [http://www5.statcan.gc.ca/cansim/a01?lang=eng Last accessed: August 18, 2011] (2011).

[126] G. Guerrero-Mora, P. Elvia, A. Bianchi, J. Kortelainen, M. Tenhunen, S. Himanen, M. Mendez, E. Arce-Santana, and O. Gutierrez-Navarro. "Sleep-wake detection based on respiratory signal acquired through a pressure bed sensor." In "Proc. 2012 Ann. Int. Conf. IEEE Engineering in Medicine and Biology Society (EMBC 2012)," pages 3452-3455 (2012).

[127] T. Kirjavainen, D. Cooper, O. Polo, and C. Sullivan. "Respiratory and body movements as indicators of sleep stage and wakefulness in infants and young children." J. Sleep. Res. 5(3), 186-194 (1996).

[128] B. H. Jansen and K. Shankar. "Sleep staging with movement-related signals." Int. J. Bio-medical Comput. 32(3-4), 289-297 (1993).

[129] A. Zaffaroni, B. Kent, E. O'Hare, C. Heneghan, P. Boyle, G. O'Connell, M. Pallin, P. de Chazal, and W. T. McNicholas. "Assessment of sleep-disordered breathing using a non-contact bio-motion sensor." J. Sleep. Res. 22(2), 231236. ISSN 1365-2869 (2013).

[130] E. Svanborg, H. Larsson, B. Carlsson-Nordlander, and R. Pirskanen. "A limited diagnostic investigation for obstructive sleep apnea syndrome. oximetry and static charge sensitive bed." Chest 98(6), 1341-1345 (1990).

[131] A. M. Adami, A. G. Adami, T. L. Hayes, and Z. T. Beattie. "A system for assessment of limb movements in sleep." In "Proc. 2013 IEEE 15th Int. Conf. eHealth Networking, Applications Services (Healthcom)," pages 419-423 (2013).

[132] E. Rauhala, M. Erkinjuntti, and O. Polo. "Detection of periodic leg movements with a static-charge-sensitive bed." J. Sleep. Res. 5(4), 246-250 (1996).

[133] A. Arcelus, C. Herry, R. Goubran, F. Knoefel, H. Sveistrup, and M. Bilodeau. "Determination of sit-to-stand transfer duration using bed and floor pressure 
sequences." IEEE Trans. Biomed. Eng. 56(10), 2485-2492. ISSN 0018-9294 (2009).

[134] A. Arcelus, I. Veledar, R. Goubran, F. Knoefel, H. Sveistrup, and M. Bilodeau. "Measurements of sit-to-stand timing and symmetry from bed pressure sensors." IEEE Trans. Instrum. Meas. 60(5), 1732 -1740. ISSN 0018-9456 (2011).

[135] K. Sakai, H. Sanada, N. Matsui, G. Nakagami, J. Sugama, C. Komiyama, and N. Yahagi. "Continuous monitoring of interface pressure distribution in intensive care patients for pressure ulcer prevention." J. Adv. Nursing 65(4), 809-817. ISSN 03092402 (2009).

[136] R. Kurozumi, T. Yamamoto, S. Fujisawa, and O. Sueda. "Development of a postural supporting device using an adaptive and learning balloon actuator-sensor system." In "Proc. 2007 IEEE Ann. Conf. Soc. of Instr. Control Engineers," pages 488-493 (2007).

[137] R. D. Howe. "Tactile sensing and control of robotic manipulation." Adv Robot 8(3), 245-261 (1994).

[138] K. Sakai, G. Nakagami, N. Matsui, H. Sanada, A. Kitagawa, E. Tadaka, and J. Sugama. "Validation and determination of the sensing area of the KINOTEX sensor as part of development of a new mattress with an interface pressuresensing system." Biosci. Trends 2(1), 36-43. ISSN 1881-7823 (2008).

[139] H. L. Watson, D. A. Poole, and M. A. Sackner. "Accuracy of respiratory inductive plethysmographic cross-sectional areas." J. Appl. Physiol. 65(1), 306 -308 (1988).

[140] K. P. Cohen, W. M. Ladd, D. M. Beams, W. S. Sheers, R. G. Radwin, W. J. Tompkins, and J. G. Webster. "Comparison of impedance and inductance ventilation sensors on adults during breathing, motion, and simulated airway obstruction." IEEE Trans. Biom. Eng. 44(7), 555-566 (1997).

[141] P. Valta, J. Takala, R. Foster, C. Weissman, and J. M. Kinney. "Evaluation of respiratory inductive plethysmography in the measurement of breathing pattern and PEEP-induced changes in lung volume." Chest 102(1), 234 -238 (1992).

[142] P.-Y. Carry, P. Baconnier, A. Eberhard, P. Cotte, and G. Benchetrit. "Evaluation of respiratory inductive plethysmography : Accuracy for analysis of respiratory waveforms." Chest 111(4), 910-915. ISSN 0012-3692 (1997). 
[143] K. F. Whyte, M. Gugger, G. A. Gould, J. Molloy, P. K. Wraith, and N. J. Douglas. "Accuracy of respiratory inductive plethysmograph in measuring tidal volume during sleep." J. Appl. Physiol. 71(5), 1866 -1871 (1991).

[144] P. V. Zimmerman, S. J. Connellan, H. C. Middleton, M. V. Tabona, M. D. Goldman, and N. Pride. "Postural changes in rib cage and abdominal volumemotion coefficients and their effect on the calibration of a respiratory inductance plethysmograph." Am. Rev. Respir. Dis 127(2), 209-214. ISSN 0003-0805 (1983).

[145] R. B. Berry, R. Budhiraja, D. J. Gottlieb, D. Gozal, C. Iber, V. K. Kapur, C. L. Marcus, R. Mehra, S. Parthasarathy, S. F. Quan, S. Redline, K. P. Strohl, S. L. D. Ward, and M. M. Tangredi. "Rules for scoring respiratory events in sleep: Update of the 2007 AASM manual for the scoring of sleep and associated events." J Clin Sleep Med 8(5), 597-619. ISSN 1550-9389 (2012).

[146] J. L. Rodgers and W. A. Nicewander. "Thirteen ways to look at the correlation coefficient." The American Statistician 42(1), 59-66. ISSN 0003-1305 (1988).

[147] H. Cooper, L. V. Hedges, and J. C. Valentine. The Handbook of Research Synthesis and Meta-Analysis. Russell Sage Foundation. ISBN 9781610441384 (2009).

[148] R. A. Fisher. "Frequency distribution of the values of the correlation coefficient in samples from an indefinitely large population." Biometrika 10(4), 507-521. ISSN 0006-3444 (1915).

[149] J. L. Devore. Probability and statistics for engineering and the sciences. Duxbury Press, Belmont, USA, 4th edition. ISBN 0-534-24264-2 (1995).

[150] J. E. Hunter and F. L. Schmidt. Methods of Meta-Analysis: Correcting Error and Bias in Research Findings. SAGE. ISBN 9781412904797 (2004).

[151] D. J. Saville. "Multiple comparison procedures: The practical solution." Am. Stat. 44(2), 174-180. ISSN 0003-1305, 1537-2731 (1990).

[152] P. A. D. Wilks and M. J. English. "Accurate segmentation of respiration waveforms from infants enabling identification and classification of irregular breathing patterns." Med. Eng. Phys. 16(1), 1923 (1994).

[153] J. M. Bland and D. G. Altman. "Statistical methods for assessing agreement between two methods of clinical measurement." Lancet 327(8476), 307-310. ISSN 0140-6736 (1986). 
[154] B. L. Gray and R. S. Fearing. "A surface micromachined microtactile sensor array." In "Proc. 1996 IEEE Int. Conf. Robotics and Automation," volume 1, pages $1-6$ (1996).

[155] J. Dargahi and S. Najarian. "An endoscopic force-position sensor grasper with minimum sensors." Can. J. Electr. Comp. Eng. Rev. Can. Genie. Electr. Inform. 28(3), 155-161 (2003).

[156] A. Desai, S. Payandeh, and J. Vaisey. "On the localization of objects using an FSR pressure pad transducer." In "Proc. 1994 IEEE Int. Conf. Systems, Man, and Cybernetics (SMC 1994)," volume 1, pages 953-957 (1994).

[157] M. Ueda, S. Mizuno, A. Matsumura, and F. Tohjyo. "Light attenuation in a semitransparent foam sheet-thickness measurement for industrial use." Opt. Laser Eng. 24(4), 339-350. ISSN 0143-8166 (1996).

[158] E. J. Kuncir, R. W. Wirta, and F. L. Golbranson. "Load-bearing characteristics of polyethylene foam: an examination of structural and compression properties." J. Rehabil. Res. Dev. 27(3), 229-238 (1990).

[159] J. C. Patra, A. C. Kot, and G. Panda. "An intelligent pressure sensor using neural networks." IEEE Trans. Instrum. Meas. 49(4), 829-834 (2000).

[160] B. A. Staats, H. W. Bonekat, C. D. Harris, and K. P. Offord. "Chest wall motion in sleep apnea." Am. Rev. Respir. Dis 130(1), 59-63. ISSN 0003-0805 (1984).

[161] G. K. Prisk, J. Hammer, and C. J. Newth. "Techniques for measurement of thoracoabdominal asynchrony." Pediatr. Pulmonol. 34(6), 462-472. ISSN 87556863 (2002).

[162] J. Proakis, J. Drouilhet, P.R., and R. Price. "Performance of coherent detection systems using decision-directed channel measurement." IEEE Trans. Commun. Syst. 12(1), 54-63. ISSN 0096-1965 (1964).

[163] K.-G. Wu and J.-A. Wu. "Efficient decision-directed channel estimation for OFDM systems with transmit diversity." IEEE Commun. Lett. 15(7), 740742. ISSN 1089-7798 (2011).

[164] J. Sohn, N. S. Kim, and W. Sung. "A statistical model-based voice activity detection." IEEE Signal Processing Lett. 6(1), 1-3. ISSN 1070-9908 (1999). 
[165] R. McAulay and E. Denlinger. "A decision - directed adaptive tracker." IEEE Trans. Aerosp. Electron. Syst. AES-9(2), 229-236. ISSN 0018-9251 (1973).

[166] M. H. Hayes. Statistical Digital Signal Processing and Modeling. John Wiley \& Sons, Inc. New York, NY, USA (1996).

[167] D. R. Cox and A. Stuart. "Some quick sign tests for trend in location and dispersion." Biometrika 42(1/2), 80-95. ISSN 00063444 (1955).

[168] T. Moyles, R. Erlandson, and T. Roth. "A nonparametric statistical approach to breath segmentation." In "Proc. 1989 Ann. Int. Conf. IEEE Engineering in Medicine and Biology Society (EMBC 1989)," volume 1, pages 330-331 (1989).

[169] J. Hammer and C. J. L. Newth. "Assessment of thoraco-abdominal asynchrony." Paediatr. Respir. Rev. 10(2), 75-80. ISSN 1526-0550 (2009).

[170] T. S. Rappaport. Wireless communications: principles and practice. Prentice Hall PTR. ISBN 9780133755367 (1996).

[171] M. Joho, H. Mathis, and R. H. Lambert. "Overdetermined blind source separation: Using more sensors than source signals in a noisy mixture." In "Proc. ICA," page 8186 (2000).

[172] T. G. Dietterich. "Ensemble methods in machine learning." In "Multiple Classifier Systems," Number 1857 in Lecture Notes in Computer Science, pages 1-15. Springer Berlin Heidelberg. ISBN 978-3-540-67704-8, 978-3-540-45014-6 (2000).

[173] D. Parikh and R. Polikar. "An ensemble-based incremental learning approach to data fusion." IEEE Transactions on Systems, Man, and Cybernetics, Part B: Cybernetics 37(2), 437-450. ISSN 1083-4419 (2007).

[174] K. Muller, S. Mika, G. Ratsch, K. Tsuda, and B. Scholkopf. "An introduction to kernel-based learning algorithms." IEEE Trans. Neur. Net. 12(2), 181-201. ISSN 1045-9227 (2001).

[175] C. J. C. Burges. "A tutorial on support vector machines for pattern recognition." Data Min. Knowl. Discov. 2(2), 121-167. ISSN 1384-5810, 1573-756X (1998).

[176] C. Cortes and V. Vapnik. "Support-vector networks." Mach. Learn. 20(3), 273-297. ISSN 0885-6125, 1573-0565 (1995). 
[177] C.-W. Hsu, C.-C. Chang, and C.-J. Lin. A practical guide to support vector classification. [https://www.cs.sfu.ca/people/Faculty/teaching/726/spring11/svmguide.pdf] (2003).

[178] C.-C. Chang and C.-J. Lin. "LIBSVM: a library for support vector machines." ACM Trans. Intell. Syst. Technol. 2(3), 27:127:27. Software available at http://www.csie.ntu.edu.tw/ cjlin/libsvm (2011).

[179] Y. Bengio and Y. Grandvalet. No unbiased estimator of the variance of K-fold cross-validation. CIRANO (2003).

[180] F. Melgani and Y. Bazi. "Classification of electrocardiogram signals with support vector machines and particle swarm optimization." IEEE Trans. Inf. Technol. Biomed. 12(5), 667-677. ISSN 1089-7771 (2008).

[181] J. Robinson and Y. Rahmat-Samii. "Particle swarm optimization in electromagnetics." IEEE Trans. Antennas Propagat. 52(2), 397-407. ISSN 0018-926X (2004).

[182] M. Holtzman, F. Knoefel, J. Pereira, and R. Goubran. "Unobtrusive monitoring of respiratory variability in palliative care: A small case series." In "Proc. Int. Congress on Palliative Care," volume 28, pages 219-219 (2012).

[183] D. S. Lauderdale, K. L. Knutson, L. L. Yan, P. J. Rathouz, S. B. Hulley, S. Sidney, and K. Liu. "Objectively measured sleep characteristics among earlymiddle-aged adults: the CARDIA study." Am. J. Epidemiol. 164(1), 5-16. ISSN 0002-9262 (2006).

[184] R. C. Petersen. "Mild cognitive impairment." New Engl. J. Med. 364(23), 2227-2234. ISSN 0028-4793 (2011).

[185] S. M. McCurry and S. Ancoli-Israel. "Sleep dysfunction in alzheimers disease and other dementias." Curr. Treat Options Neurol. 5(3), 261-272. ISSN 10928480, 1534-3138 (2003).

[186] A. Bianchi, M. Mendez, and S. Cerutti. "Processing of signals recorded through smart devices: Sleep-quality assessment." IEEE Trans. Inf. Technol. Biomed. 14(3), 741-747. ISSN 1089-7771 (2010).

[187] A. Loerbroks, D. Debling, M. Amelang, and T. Strmer. "Nocturnal sleep duration and cognitive impairment in a population-based study of older adults." Int. J. Geriatr. Psychiatry 25(1), 100-109. ISSN 1099-1166 (2010). 
[188] T. Auyeung, J. Lee, J. Leung, T. Kwok, P. Leung, J. Woo, and Y. Wing. "Cognitive deficit is associated with phase advance of sleepwake rhythm, daily napping, and prolonged sleep durationa cross-sectional study in 2,947 communitydwelling older adults." Age (Dordr) 35(2), 479-486. ISSN 0161-9152 (2013).

[189] D. Townsend, R. Goubran, F. Knoefel, and J. Leech. "Validation of unobtrusive pressure sensor array for central sleep apnea screening." IEEE Trans. Instrum. Meas. 61(7), 1857 -1865. ISSN 0018-9456 (2012).

[190] K. M. Rose, C. Beck, P.-F. Tsai, P. H. Liem, D. G. Davila, M. Kleban, N. S. Gooneratne, G. Kalra, and K. C. Richards. "Sleep disturbances and nocturnal agitation behaviors in older adults with dementia." Sleep 34(6), 779-786. ISSN 0161-8105 (2011).

[191] N. T. Aggarwal. "Motor dysfunction in mild cognitive impairment and the risk of incident alzheimer disease." Arch. Neurol. 63(12), 1763. ISSN 0003-9942 (2006).

[192] J. Verghese, C. Wang, R. B. Lipton, R. Holtzer, and X. Xue. "Quantitative gait dysfunction and risk of cognitive decline and dementia." J. Neurol. Neurosurg. Psychiatry 78(9), 929-935. ISSN , 1468-330X (2007).

[193] P. A. Boyle, R. S. Wilson, A. S. Buchman, N. T. Aggarwal, Y. Tang, Z. Arvanitakis, J. Kelly, and D. A. Bennett. "Lower extremity motor function and disability in mild cognitive impairment." Exp. Aging Res. 33(3), 355-371. ISSN 0361-073X (2007).

[194] E. H. Franssen, L. E. Souren, C. L. Torossian, and B. Reisberg. "Equilibrium and limb coordination in mild cognitive impairment and mild alzheimer's disease." J. Am. Geriatr. Soc. 47(4), 463-469. ISSN 0002-8614 (1999).

[195] C. Annweiler, A. Schott, G. Abellan Van Kan, Y. Rolland, H. Blain, B. Fantino, F. Herrmann, and O. Beauchet. "The five-times-sit-to-stand test, a marker of global cognitive functioning among community-dwelling older women." J. Nutr. Health Aging 15(4), 271-276. ISSN 1279-7707 (2011).

[196] M. E. Taylor, K. Delbaere, S. R. Lord, A. S. Mikolaizak, and J. C. T. Close. "Physical impairments in cognitively impaired older people: implications for risk of falls." Int. Psychogeriatr. 25(1), 148-156. ISSN 1741-203X (2012).

[197] A. P. Spira, T. Blackwell, K. L. Stone, S. Redline, J. A. Cauley, S. Ancoli-Israel, and K. Yaffe. "Sleep-disordered breathing and cognition in older women." $J$. Am. Geriatr. Soc. 56(1), 4550. ISSN 1532-5415 (2008). 
[198] S. Ancoli-Israel, B. W. Palmer, J. R. Cooke, J. Corey-Bloom, L. Fiorentino, L. Natarajan, L. Liu, L. Ayalon, F. He, and J. S. Loredo. "Cognitive effects of treating obstructive sleep apnea in alzheimer's disease: A randomized controlled study." J. Am. Geriatr. Soc. 56(11), 2076-2081. ISSN 1532-5415 (2008).

[199] S. Ancoli-Israel, P. Clopton, M. R. Klauber, R. Fell, and W. Mason. "Use of wrist activity for monitoring Sleep/Wake in demented nursing-home patients." Sleep 20(1), 24-27. ISSN 0161-8105 (1997).

[200] N. Wolkove, O. Elkholy, M. Baltzan, and M. Palayew. "Sleep and aging: 1. sleep disorders commonly found in older people." CMAJ 176(9), 1299-1304. ISSN 0820-3946, 1488-2329 (2007).

[201] W. W. Flemons, N. J. Douglas, S. T. Kuna, D. O. Rodenstein, and J. Wheatley. "Access to diagnosis and treatment of patients with suspected sleep apnea." Am. J. Respir. Crit. Care Med. 169(6), 668-672. ISSN 1073-449X, 1535-4970 (2004).

[202] T. Morgenthaler, C. Alessi, L. Friedman, J. Owens, V. Kapur, B. Boehlecke, T. Brown, J. Chesson, Andrew, J. Coleman, T. Lee-Chiong, J. Pancer, and T. J. Swick. "Practice parameters for the use of actigraphy in the assessment of sleep and sleep disorders: an update for 2007." Sleep 30(4), 519-529. ISSN 0161-8105 (2007).

[203] A. Sadeh. "The role and validity of actigraphy in sleep medicine: an update." Sleep Med. Rev. 15(4), 259-267. ISSN 1532-2955 (2011).

[204] M. Hoekert, R. F. R.-v. der Lek, D. F. Swaab, D. Kaufer, and E. J. W. Van Someren. "Comparison between informant-observed and actigraphic assessments of SleepWake rhythm disturbances in demented residents of homes for the elderly." Am. J. Geriatr. Psychiatry 14(2), 104-111. ISSN 1064-7481 (2006).

[205] I. Higginson and G. Sen-Gupta. "Place of care in advanced cancer: A qualitative systematic literature review of patient preferences." J. Palliat. Med. 3(3), 287300. ISSN 1096-6218 (2000).

[206] B. Gomes and I. J. Higginson. "Factors influencing death at home in terminally ill patients with cancer: systematic review." BMJ 332(7540), 515-521. ISSN 0959-8138, 1468-5833 (2006). 
[207] R. J. Dunlop, R. J. Davies, and J. M. Hockley. "Preferred versus actual place of death: a hospital palliative care support team experience." Palliative Med. 3(3), $197-201$ (1989).

[208] J. Townsend, A. O. Frank, D. Fermont, S. Dyer, O. Karran, A. Walgrove, and M. Piper. "Terminal cancer care and patients' preference for place of death: a prospective study." BMJ 301(6749), 415-417. ISSN 0959-8138 (1990).

[209] S. J. Goodlin. "Palliative care in congestive heart failure." J. Am. Coll. Cardiol. 54(5), 386-396. ISSN 0735-1097 (2009).

[210] J. E. S. Nolte, F. Dette, W. Cassel, C. Riese, M. Augsten, and U. Koehler. "Long-term opioid therapy and respiratory insufficiency during sleep." Pneumologie 64(4), 241-245. ISSN 1438-8790 (2010).

[211] R. G. Miller, C. E. Jackson, E. J. Kasarskis, J. D. England, D. Forshew, W. Johnston, S. Kalra, J. S. Katz, H. Mitsumoto, J. Rosenfeld, C. Shoesmith, M. J. Strong, and S. C. Woolley. "Practice parameter update: The care of the patient with amyotrophic lateral sclerosis: Drug, nutritional, and respiratory therapies (an evidence-based review) report of the quality standards subcommittee of the american academy of neurology." Neurology 73(15), 1218-1226. ISSN 0028-3878, 1526-632X (2009).

[212] C. de Miguel Snchez, S. G. Elustondo, A. Estirado, F. V. Snchez, C. G. de la Rasilla Cooper, A. L. Romero, A. Otero, and L. G. Olmos. "Palliative performance status, heart rate and respiratory rate as predictive factors of survival time in terminally ill cancer patients." J. Pain Symptom Manage. 31(6), 485492. ISSN 0885-3924 (2006).

[213] F. Domeisen Benedetti, C. Ostgathe, J. Clark, M. Costantini, M. L. Daud, B. Grossenbacher-Gschwend, R. Latten, O. Lindqvist, A. Peternelj, S. Schuler, K. Tal, A. van der Heide, and S. Eychmller. "International palliative care experts' view on phenomena indicating the last hours and days of life." Support Care Cancer 21(6), 1509-1517. ISSN 1433-7339 (2012).

[214] M. T. La Rovere, G. D. Pinna, R. Maestri, E. Robbi, A. Mortara, F. Fanfulla, O. Febo, and P. Sleight. "Clinical relevance of short-term day-time breathing disorders in chronic heart failure patients." Eur. J. Heart Fail. 9(9), 949 (2007).

[215] E. Bruera, M. J. Miller, N. Kuehn, T. MacEachern, and J. Hanson. "Estimate of survival of patients admitted to a palliative care unit: A prospective 
study." Journal of Pain and Symptom Management 7(2), 82-86. ISSN 08853924 (1992).

[216] S. Inoue, K. Hayashida, M. Nakamura, Y. Nohara, and N. Nakashima. "Capturing nursing interactions from mobile sensor data and in-room sensors." In A. Marcus, editor, "Design, User Experience, and Usability. User Experience in Novel Technological Environments," Number 8014 in Lecture Notes in Computer Science, pages 280-289. Springer Berlin Heidelberg. ISBN 978-3-64239237-5, 978-3-642-39238-2 (2013).

[217] S. F. Quan, J. C. Gillin, M. R. Littner, and J. W. Shepard. "Sleep-related breathing disorders in adults: Recommendations for syndrome definition and measurement techniques in clinical research." Sleep 22(5), 662-689. ISSN 01618105 (1999).

[218] O. E. Dictionary. "palliative, adj. and n." (2011).

[219] S. Carstairs, C. E. L. (Firm), and C. P. Senate. Raising the bar: A roadmap for the future of palliative care in Canada. Senate of Canada (2010).

[220] D. Clark. "From margins to centre: a review of the history of palliative care in cancer." Lancet Oncol. 8(5), 430-438. ISSN 1470-2045 (2007).

[221] C. Seplveda, A. Marlin, T. Yoshida, and A. Ullrich. "Palliative care: the world health organization's global perspective." J. Pain Symptom Manage. 24(2), 91-96. ISSN 0885-3924 (2002).

[222] R. S. Morrison, J. D. Penrod, J. B. Cassel, M. Caust-Ellenbogen, A. Litke, L. Spragens, D. E. Meier, and for the Palliative Care Leadership Centers' Outcomes Group. "Cost savings associated with US hospital palliative care consultation programs." Arch. Intern. Med. 168(16), 1783-1790 (2008).

[223] G. Gade, I. Venohr, D. Conner, K. McGrady, J. Beane, R. H. Richardson, M. P. Williams, M. Liberson, M. Blum, and R. D. Penna. "Impact of an inpatient palliative care team: A randomized controlled trial." J. Palliat. Med. 11(2), 180-190. ISSN 1096-6218 (2008).

[224] S. C. Hospice. "History | st christopher's hospice." [http://www.stchristophers.org.uk/about/history Last accessed: 2011-0801 16:52:16 ] (2011).

[225] H. B. Wodinsky. "Palliative care in canada: An overview." Am. J. Of Hospice And Palliat. 6(6), $44-47$ (1989). 
[226] C. Zimmermann, D. Seccareccia, A. Clarke, D. Warr, and G. Rodin. "Bringing palliative care to a canadian cancer center: the palliative care program at princess margaret hospital." Support Care Cancer 14(10), 982-987. ISSN 0941-4355 (2006).

[227] D. E. Meier and O. W. Brawley. "Palliative care and the quality of life." J. Clin. Oncol. 29(20), 2750-2752. ISSN 1527-7755 (2011).

[228] S. Dalal, S. Palla, D. Hui, L. Nguyen, R. Chacko, Z. Li, N. Fadul, C. Scott, V. Thornton, B. Coldman, Y. Amin, and E. Bruera. "Association between a name change from palliative to supportive care and the timing of patient referrals at a comprehensive cancer center." Oncologist 16(1), 105 -111 (2011).

[229] R. Brumley, S. Enguidanos, P. Jamison, R. Seitz, N. Morgenstern, S. Saito, J. McIlwane, K. Hillary, and J. Gonzalez. "Increased satisfaction with care and lower costs: Results of a randomized trial of in-home palliative care." J. Am. Geriatr. Soc. 55(7), 993-1000. ISSN 00028614 (2007).

[230] P. J. Franks, C. Salisbury, N. Bosanquet, E. K. Wilkinson, S. Kite, A. Naysmith, and I. J. Higginson. "The level of need for palliative care: a systematic review of the literature." J. Palliat. Med. 14(2), 93 -104 (2000).

[231] P. Hudson. "Home-based support for palliative care families: Challenges and recommendations." Med. J. Aust. 179(6), S35-S37. ISSN 0025-729X (2003).

[232] P. Hall, C. Schroder, and L. Weaver. "The last 48 hours of life in long-term care: A focused chart audit." J. Am. Geriatr. Soc. 50(3), 501-506. ISSN 00028614 (2002).

[233] E. Bruera, J. Pereira, S. Watanabe, M. Belzile, N. Kuehn, and J. Hanson. "Opioid rotation in patients with cancer pain: A retrospective comparison of dose ratios between methadone, hydromorphone, and morphine." Cancer 78(4), 852-857 (1996).

[234] C. A. Kushida, M. R. Littner, T. Morgenthaler, C. A. Alessi, D. Bailey, J. C. Jr, L. Friedman, M. Hirshkowitz, S. Kapen, M. Kramer, T. Lee-Chiong, D. L. Loube, J. Owens, J. P. Pancer, and M. Wise. "Practice parameters for the indications for polysomnography and related procedures: an update for 2005." Sleep 28(4), 499-521 (2005).

[235] G. D. Pinna, R. Maestri, A. Mortara, P. Johnson, D. Andrews, P. Ponikowski, T. Witkowski, E. Robbi, M. T. La Rovere, and P. Sleight. "Pathophysiological 
and clinical relevance of simplified monitoring of nocturnal breathing disorders in heart failure patients." Eur. J. Heart Fail. 11(3), 264-272. ISSN 1388-9842 (2009).

[236] K. Brazil, D. Howell, M. Bedard, P. Krueger, and C. Heidebrecht. "Preferences for place of care and place of death among informal caregivers of the terminally ill." J. Palliat. Med. 19(6), $492-499$ (2005).

[237] J. Cohen, D. Houttekier, B. Onwuteaka-Philipsen, G. Miccinesi, J. AddingtonHall, S. Kaasa, J. Bilsen, and L. Deliens. "Which patients with cancer die at home? a study of six european countries using death certificate data." J. Clin. Oncol 28(13), 2267-2273. ISSN 1527-7755 (2010).

[238] B. Gomes and I. J. Higginson. "Where people die (19742030): past trends, future projections and implications for care." J. Palliat. Med. 22(1), $33-41$ (2008).

[239] B. Gomes, P. McCrone, S. Hall, J. Koffman, and I. J. Higginson. "Variations in the quality and costs of end-of-life care, preferences and palliative outcomes for cancer patients by place of death: the QUALYCARE study." $B M C$ Cancer 10, 400-400 (2010).

[240] D. Hui, A. Elsayem, M. De La Cruz, A. Berger, D. S. Zhukovsky, S. Palla, A. Evans, N. Fadul, J. L. Palmer, and E. Bruera. "Availability and integration of palliative care at US cancer centers." JAMA 303(11), $1054-1061$ (2010). 


\section{Appendix A}

\section{Background Information on Palliative Care}

Although the concept of palliative care is known to many, it is a slightly nebulous idea to those who have not had direct contact with it. This is not simply a lack of common knowledge, but is partly due to the relative youth of a field that continues to evolve and redefine its role. Learning the history of palliative care enlightens its current role and so this history is provided in this appendix. By understanding the role of palliative care and its goals, engineers working with palliative care can better orient their systems to help patients, caregivers, support workers, and physicians.

This appendix introduces the reader to the palliative care environment, including a short history of the field and its newer trends, a description of palliative care clients and their symptoms, and the emergence of palliative home care.

\section{A.1 Overview of Palliative Care}

The adjective 'palliative' describes to "...relieve the symptoms of a disease or condition without dealing with the underlying cause" [218]. Globally, of 56 million deaths per year, $60 \%$ could make use of palliative care $90 \%$ of Canadians could (eventually) 
benefit from palliative care services [219], but currently only a small fraction do [220]. The main goal of palliative care is to improve the quality of life of patients. The World Health Organization (WHO) has defined palliative care as:

"an approach that improves improves the quality of life of patients and their families facing the problems associated with life-threatening illness, through the prevention and relief of suffering by means of early identification and impeccable assessment and treatment of pain and other problems, physical, psychosocial and spiritual." [221].

Embedded in this definition are a number of important principles of palliative care. Firstly, that palliative care is concerned with quality of life, which involves not only physical ailment, but also psychological, social, and spiritual problems. Secondly, palliative care encompasses both the patient and the family. Family members may require counselling and ultimately bereavement support. Since family often provides the main care giving for the patient, they also require care giving resources and support. Finally, palliative care is provided to people facing any life-threatening illness, and not just for those who have been diagnosed as terminal.

Palliative care has been shown to lower the total cost of hospital admissions for adults with serious illnesses, due to decreased use of laboratory and intensive care services [222]. Total hospital costs following discharge are also reported to be lower [223].

\section{Short History Of Palliative Medicine}

Palliative care is one of the newest fields of medicine. Up to the 1960's, incurable patients were usually ignored by the medical profession since the search for curative treatments was the dominant concern [220]. Pain relief for these incurable patients was rare. The most effective pain relief drugs, such as morphine, were thought to 
be too addictive and dangerous [224]. Studies into terminal care in cancer in the 1950's and 1960's, spearheaded by Dame Cicely Mary Saunders, formed the first interest in the role of palliative care by the medical profession. The first modern hospice was opened in London, England in 1967. Although other hospices existed, mainly run by religious groups [220], St. Christopher's Hospice was the first to include science, education, and research with care for the patients' physical, spiritual, and psychological well-being [224].

Palliative care did not reach Canada until 1975, when two hospitals, one in Winnipeg and one in Montreal, started operating palliative care services [225]. Although palliative care services have grown across the country, a senate report on the future of palliative care published in 2010 found that $70 \%$ of Canadians are still without access to palliative care [219].

\section{New Trends in Palliative Care}

While palliative care began as end-stage care, it is now being provided earlier, even while curative treatments are still being sought [226]. The WHO has shifted its definition of palliative care to start "as early as possible" for any chronic fatal illness, rather than during the last stages of care [221]. This shift improves patient care, since palliative care has been shown to improve patient outcomes in cancer, perhaps due to improving immune reserves that get depleted by the stressors of serious illness [227]. Additionally, early pain management prevents the nervous system from building neural pathways that reinforce pain transmission [221]. Not only do the patients benefit from palliative care, but their caregivers may also experience better health outcomes, as they too are relieved of some immunity-depleting stressors [227].

The provision of palliative care to any patient facing a potentially fatal illness has also led to a call for a name change. Recent research has shown that changing the name to "supportive care" dramatically expands patient use during early stage 
cancers, mainly from increased and earlier physician referrals [228]. Alongside name changes, distinctions between palliative care and hospice (end-of-life) care are being encouraged [227].

Palliative home care is growing and recent research points to better satisfaction and lower costs of providing home care than institutional care [229]. Accommodating ongoing palliative care at home, and not just near the end of life, is progressing. Further specifics of palliative home care are discussed in Section A.4.

\section{A.2 Palliative Care Clients}

Patients in palliative care face a wide range of disease diagnoses, including all types of cancer, congestive heart failure $(\mathrm{CHF})$, dementia, chronic obstructive pulmonary disease (COPD), and acquired immune deficiency syndrome (AIDS). These may be categorized into three types: cancers, progressive nonmalignant disease, and children's terminal illnesses [230]. With such a wide range of diseases, treatments cannot be generalized. However, common elements exist in care and form the basis for palliative care services.

As explored previously in Section A.1, clients of palliative care services include not only the patients themselves, but also their family and other care providers. In an institutional setting, "caregiver" refers to staff, but in most cases the most important caregivers are family or friends of the patient. Home care responsibility can include performing the regular day to day duties such as cooking, shopping or housework, but can also include more advanced nursing care such as catheter changing or opioid administration [231]. Clearly, both technical and psychosocial support is needed for these caregivers. Technologies that are introduced in home care settings must be easily used by those unfamiliar or new to the technology. 


\section{A.3 End of Life Symptoms}

Some common symptoms are present across palliative care patients. Pain is an important symptom, but will not be addressed here, since its relief using bed-based sensing is currently not foreseen, except perhaps in preventing pressure sores from long term bed rest. Two important palliative care symptoms are breathing disorders and delirium.

\section{Disordered Breathing Patterns}

Breathing related disorders are amongst the most prevalent symptom in palliative care patients at the end of life [232]. In patients with chronic heart failure (CHF), the presence of periodic breathing is correlated with mortality [214]. The quality of life for patients with disordered breathing could be improved by interventions such as nocturnal oxygen therapy or continuous positive airway pressure therapy [209] or non-invasive repositioning [11]. Although opioids, when used appropriately, are safe, respiratory depression may occur at high doses of opioids and sedatives [233]. With early identification of drug induced respiratory complications, adjustments can be made to the treatments [210].

Many disordered breathing patterns can occur at the end of life. They can be grouped into apneas, rapid breathing, and oscillatory breathing. Apnea is the cessation of breath. There are two main types of apnea: central apnea and obstructive apnea. During central apneas, the brain stem does not send the neural signals to the lungs to initiate breathing. There is a complete lack of breathing effort. During obstructive apneas, breathing effort exists, but constrictions (i.e. obstructions) in the air passage inhibit air exchange in the lungs. There is also a third related category of disordered breathing, hypopnea. Hypopnea is characterized by low amplitude breathing that does not allow enough oxygen and carbon dioxide exchange [234]. 
The apnea hypopnea index (AHI), also termed the respiratory disturbance index (RDI), is often used to quantify breathing disorder. AHI is the number of apneas and hypopneas per hour during overnight polysomnography testing, although its exact definition may change depending on the study [11]. AHI may not take into account respiratory disturbance from other amplitude or rate changing patterns.

Oscillatory breathing refers to breathing patterns whereby the amplitude of the breathing volumes varies in crescendo and decrescendo pattern: each breath gets deeper and deeper and then breathing depth peaks and gets shallower and shallower. This cycle is repeated many times. Two types of oscillatory breathing are defined: periodic breathing and Cheyne-Stokes respiration.

Periodic breathing is the sustained oscillation of breathing tidal volumes. Another term for periodic breathing is "cyclical respiration". Oscillations must reach a target variation between peak and trough volume of at least $25 \%$ reduction between peak and trough values [235], [214]. However, the definition of what constitutes "sustained" varies. For instance, it has been defined as greater or equal to three minutes [235], and has also been defined as oscillations during greater or equal to $75 \%$ of the time in a 10 minute period [214]. Cheyne-Stokes respiration is similar to periodic breathing, but includes apneic episodes during the troughs.

\section{A.4 Palliative Home Care}

The majority of terminal care patients prefer to spend their last days at home $(53 \%$ [207], 58\% [208], 63\% [236]). Generally, hospitals comply with these wishes, but sometimes patients are too sick to be transferred or do not have caregivers who can cope with home care [207], [231]. Currently, only 13\%-45\% of Europeans with cancer die at home [237], although this may be higher in Canada (68\%) [236]. In some parts of the world, there is a trend towards more institutional deaths and less home 
deaths [238] [239], but again this may be reversed in North America [239].

Patient preferences for place of ongoing care also show that the majority would prefer home care to institutional care [205]. However, outpatient palliative care services (i.e. home care services) are still less prevalent than inpatient services [240]. 